\title{
Pd-catalyzed Umpolung Chemistry of Glycal Acetates and Their [2,3]-Dehydrosugar Isomers.
}

\author{
Supporting Information \\ Juyeol Lee and Young Ho Rhee* \\ Department of Chemistry, Pohang University of Science and Technology (POSTECH), Hyoja-dong \\ San 31, Pohang 790-784, Republic of Korea
}

Table of Contents

1. General information

2. Optimization of allylation with alkylaldehyde

3. Synthesis of Acetate substrates 1b, 3, 15, 17, 18, 21, 23

4. Stereoselective Synthesis of $C$-Glycoside 2a, 4-14, 16, 22, 24

5. Formal synthesis of mucocin

6. Determination of Absolute configuration of compound 2a, 22, 24 and 34

7. Comparison NMR data with natural product

8. Reference

9. ${ }^{1} \mathrm{H}$ and ${ }^{13} \mathrm{C}$ NMR of new compounds

10. ${ }^{1} \mathrm{H}$ NMR of Crude mixture: Determination of d.r for the reaction 


\section{General information}

Air and moisture sensitive reactions were carried out in oven-dried glassware sealed with rubber septa under a positive pressure of nitrogen. Similarly all solvents were dried and distilled according to the standard methods before use, then were transferred via syringe. Reactions were stirred using Teflon-coated magnetic stir bars and heated in an oil bath. $\mathrm{Pd}_{2}(\mathrm{dba})_{3}$, the Grubbs' catalysts were purchased form a Aldrich Chemical, Strem Chemical Inc. Chiral Trost ligands were purchased from Strem Chemical Inc. and stored in glove box. Reactions were monitored by thin-layer chromatography on silicagel carried out on $0.25 \mathrm{~mm}$ E. Merck silica gel plates $(60 \mathrm{~F}-$ 254) using UV light as a visualizing agent and acidic p-anisaldehyde, and heat as developing agent. Flash chromatography on silicagel was carried out on Merck 60 silica gel (230-400 mesh). ${ }^{1} \mathrm{H}$ and ${ }^{13} \mathrm{C}$ NMR spectra were recorded on Bruker (300 MHz, $500 \mathrm{MHz}, 600 \mathrm{MHz}$ ) spectrometer. ${ }^{1} \mathrm{H}$ NMR spectra were referenced to $\mathrm{CDCl}_{3}(7.26 \mathrm{ppm})$, and reported as follows; chemical shift, multiplicity $(\mathrm{s}=$ singlet, $\mathrm{d}=$ doublet, $\mathrm{t}=$ triplet, $\mathrm{q}=$ quartet, $\mathrm{m}=$ multiplet). Chemical shifts of the ${ }^{13} \mathrm{C}$ NMR spectra were measured relative to $\mathrm{CDCl}_{3}(77.23 \mathrm{ppm})$. Infrared spectra were recorded on a Shimadzu IR-470 spectrometer. Specific rotation data were measured on JASCO P-Rudolph research analytical Autopol III. HPLC was performed with an Agilent Technologies 1220 infinity LC system. Melting points were measured on Electrothermal 9100. Mass spectral data were obtained from Organic Chemistry Research Center in Sogang university on a Bruker Ultra High Resolution ESI Q-TOF MS / MS Compact System (ESI). 


\section{Optimization of allylation with alkylaldehyde}

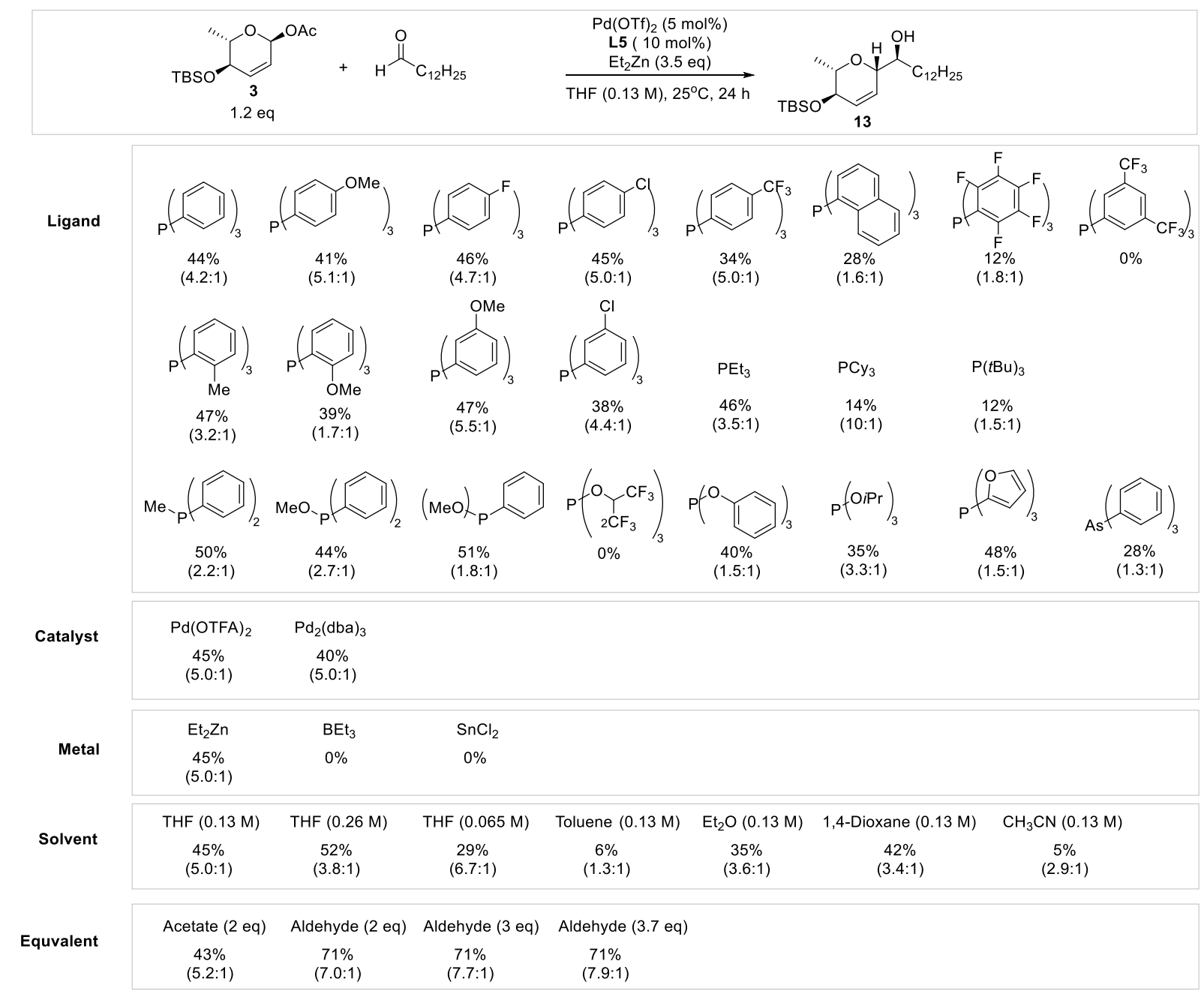




\section{Synthesis of Acetate substrates 1b, 2, 15, 17, 18, 21, 23}

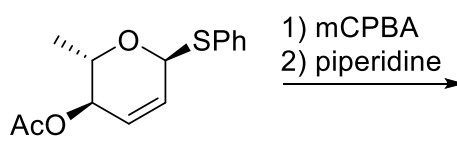<smiles>CC(=O)O[C@H]1C=CO[C@@H](C)[C@H]1O</smiles>

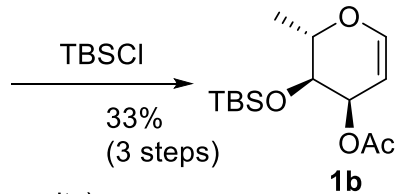

(+ $40 \%$ of inseparable impurity) $1 \mathrm{~b}$

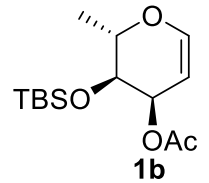

(2S,3S,4R)-3-((tert-butyldimethylsilyl)oxy)-2-methyl-3,4-dihydro-2H-pyran-4-yl acetate (1b) : To a solution of (2S,3R,6S)-2-methyl-6-(phenylthio)-3,6-dihydro-2H-pyran-3-yl acetate ${ }^{1}(1.98 \mathrm{~g}, 7.5 \mathrm{mmol})$ in $\mathrm{CH}_{2} \mathrm{Cl}_{2}(75 \mathrm{~mL})$, added mCPBA $(1.42 \mathrm{~g}, 8.25 \mathrm{mmol})$ at $-15^{\circ} \mathrm{C}$, then reaction mixture was stirred at $0^{\circ} \mathrm{C}$ for $2 \mathrm{~h}$. The mixture was quenched with sat. $\mathrm{NaHCO}_{3}$, extracted with $\mathrm{CH}_{2} \mathrm{Cl}_{2}(3 \times 50 \mathrm{~mL})$, dried over $\mathrm{Na}_{2} \mathrm{SO}_{4}$ and concentrated under reduced pressure. The crude mixture in THF $(75 \mathrm{~mL})$ was reacted with piperidine $(957 \mathrm{mg}, 11.3 \mathrm{mmol})$ at room temperature. The reaction mixture turned into yellow solution. After concentration, the crude mixture was purified by flash column chromatography (Hexane:EtOAc $=80: 20)$ to afford product with inseparable side product $(608$ $\mathrm{mg}, 40 \%$ impurity). To a solution of isolated mixture in DMF (10 mL), added imidazole (838 $\mathrm{mg}, 12.3 \mathrm{mmol})$ and $\mathrm{TBSCl}(1.24 \mathrm{~g}, 8.2 \mathrm{mmol})$ at room temperature. After stirred at $40^{\circ} \mathrm{C}$ for $18 \mathrm{~h}$, the mixture was quenched with $\mathrm{H}_{2} \mathrm{O}$, extracted with $\mathrm{Et}_{2} \mathrm{O}(4 \times 20 \mathrm{~mL})$, washed with brine $(2 \times 30 \mathrm{~mL})$, dried over $\mathrm{Na}_{2} \mathrm{SO}_{4}$ and concentrated under reduced pressure. The crude mixture was purified by flash column chromatography $\left(\mathrm{Hexane}_{\mathrm{Et}} \mathrm{O}=98: 2\right)$ to afford product $\mathbf{1 b}$ (708 $\mathrm{mg}, 2.48 \mathrm{mmol}, 33 \%$ yield) as a colorless oil.

$\mathrm{R}_{f} 0.51$ (EtOAc:Hex = 10:90); $[\alpha]^{18}{ }_{\mathrm{D}}-308.24\left(\mathrm{c}=0.73, \mathrm{CH}_{2} \mathrm{Cl}_{2}\right) ;{ }^{1} \mathrm{H}$ NMR $\left(300 \mathrm{MHz}, \mathrm{CDCl}_{3}\right) \delta 6.44(\mathrm{~d}, J=5.82$ $\mathrm{Hz}, 1 \mathrm{H}), 5.18(\mathrm{dd}, J=3.74,5.82 \mathrm{~Hz}, 1 \mathrm{H}), 4.86(\mathrm{t}, J=5.88 \mathrm{~Hz}, 1 \mathrm{H}), 4.01(\mathrm{qd}, J=6.26,10.05 \mathrm{~Hz}, 1 \mathrm{H}), 2.05(\mathrm{~s}$, $3 \mathrm{H}), 1.31(\mathrm{~d}, J=6.26 \mathrm{~Hz}, 3 \mathrm{H}), 0.88(\mathrm{~s}, 9 \mathrm{H}), 0.087(\mathrm{~s}, 3 \mathrm{H}), 0.079(\mathrm{~s}, 3 \mathrm{H}), ;{ }^{13} \mathrm{C} \mathrm{NMR}\left(75 \mathrm{MHz}, \mathrm{CDCl}_{3}\right) \delta 170.9$, 148.1, 98.0, 71.7, 71.6, 65.8, 25.9, 21.5, 18.1, 17.9, -4.37, -4.69.; IR (NaCl) v 3070, 2956, 2933, 2897, 2859, 1739 , 1645, 1471, $1370 \mathrm{~cm}^{-1}$; HRMS (ESI) m/z: [M + Na $]^{+}$Calcd for $\mathrm{C}_{14} \mathrm{H}_{26} \mathrm{O}_{4} \mathrm{SiNa}$ 309.1493; Found 309.1495.

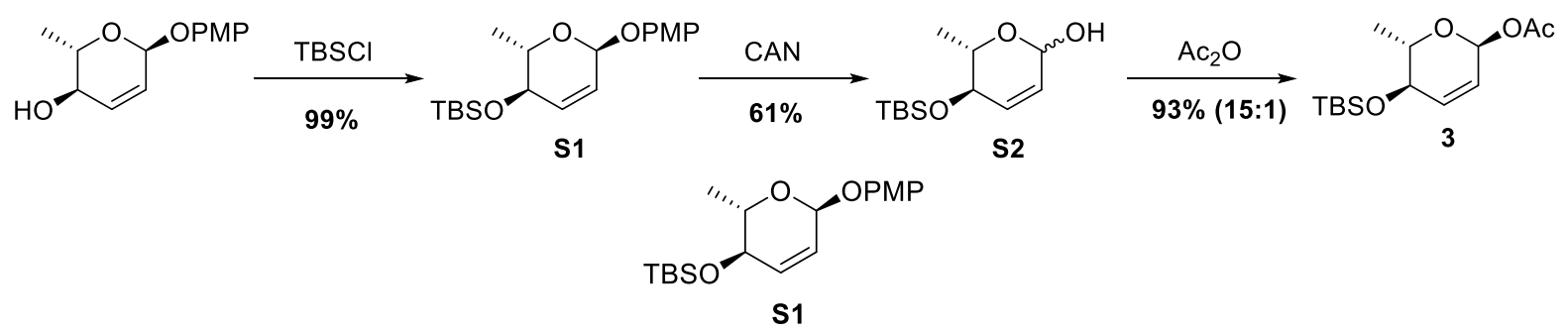

tert-butyl(((2S,3R,6S)-6-(4-methoxyphenoxy)-2-methyl-3,6-dihydro-2H-pyran-3-

yl)oxy)dimethylsilaneacetate (S1) : A solution of (2S,3R,6S)-6-(4-methoxyphenoxy)-2-methyl-3,6-dihydro-2Hpyran-3-ol ${ }^{2}(5.17 \mathrm{~g}, 21.9 \mathrm{mmol})$ in DMF $(50 \mathrm{~mL})$ was treated with imidazole $(4.47 \mathrm{~g}, 65.7 \mathrm{mmol})$ and $\mathrm{TBSCl}$ $(4.95 \mathrm{~g}, 32.9 \mathrm{mmol})$ at $0^{\circ} \mathrm{C}$. The reaction mixture was stirred at room temperature for $15 \mathrm{~h}$. The reaction mixture was quenched with $\mathrm{H}_{2} \mathrm{O}(30 \mathrm{~mL})$. The organic residue was extracted with $\mathrm{Et}_{2} \mathrm{O}(4 \times 50 \mathrm{~mL})$, wahsed with brine ( $3 \times 50 \mathrm{~mL}$ ) dried over $\mathrm{Na}_{2} \mathrm{SO}_{4}$ and concentrated under reduced pressure. The crude mixture was purified by flash column chromatography (Hexane: $\left.\mathrm{Et}_{2} \mathrm{O}=95: 5\right)$ to afford product $\mathbf{S 1}$ as colorless oil $(7.63 \mathrm{~g}, 21.8 \mathrm{mmol}, 99 \%$ yield).

$\mathrm{R}_{f} 0.41$ (EtOAc:Hex = 5:95); $[\alpha]^{15} \mathrm{D}-187.60\left(\mathrm{c}=0.86, \mathrm{CH}_{2} \mathrm{Cl}_{2}\right) ;{ }^{1} \mathrm{H}$ NMR $\left(500 \mathrm{MHz}, \mathrm{CDCl}_{3}\right) \delta 7.01-7.03(\mathrm{~m}, 2 \mathrm{H})$, 6.81-6.85 (m, 2H), $5.96(\mathrm{~d}, J=10.11 \mathrm{~Hz}, 1 \mathrm{H}), 5.82(\mathrm{ddd}, J=1.94,2.60,10.11 \mathrm{~Hz}, 1 \mathrm{H}), 5.48(\mathrm{~d}, J=1.94 \mathrm{~Hz}, 1 \mathrm{H})$, 3.91-3.92 (m, 2H), $3.79(\mathrm{~s}, 3 \mathrm{H}), 1.25(\mathrm{~d}, J=5.67 \mathrm{~Hz}, 3 \mathrm{H}), 0.91(\mathrm{~s}, 9 \mathrm{H}), 0.12(\mathrm{~s}, 3 \mathrm{H}), 0.10(\mathrm{~s}, 3 \mathrm{H}) . ;{ }^{13} \mathrm{C} \mathrm{NMR}(125$ $\left.\mathrm{MHz}, \mathrm{CDCl}_{3}\right) \delta 155.1,151.8,135.7,125.2,118.7,114.8,94.5,70.2,68.8,55.9,25.9,18.4,18.2,-3.98,-4.47$; IR 
$(\mathrm{NaCl}) \vee 3048,2955,2932,2902,2858,1507,1466,1396,1362 \mathrm{~cm}^{-1}$; HRMS (ESI) m/z: $[\mathrm{M}+\mathrm{Na}]^{+} \mathrm{Calcd}$ for $\mathrm{C}_{19} \mathrm{H}_{30} \mathrm{O}_{4} \mathrm{SiNa} 373.1806$; Found 373.1808.<smiles>CC1OC(O)C=CC1O[Mg]</smiles>

(5R,6S)-5-((tert-butyldimethylsilyl)oxy)-6-methyl-5,6-dihydro-2H-pyran-2-ol (S2) : To a solution of compound $\mathbf{S 1}(7.63 \mathrm{~g}, 21.8 \mathrm{mmol})$ in $\mathrm{CH}_{3} \mathrm{CN} / \mathrm{H}_{2} \mathrm{O}(100 \mathrm{~mL}, 4 / 1)$ at $0^{\circ} \mathrm{C}$ was added $\mathrm{NaHCO}_{3}(18.3 \mathrm{~g}, 218 \mathrm{mmol})$ and cerium ammonium nitriate $(23.9 \mathrm{~g}, 43.6 \mathrm{mmol})$. After stirring at $0^{\circ} \mathrm{C}$ for $20 \mathrm{~min}$, the reaction was quenched by sat. $\mathrm{NaHCO}_{3}$ and extracted with EtOAC $(4 \times 50 \mathrm{~mL})$ and wahsed with brine $(100 \mathrm{~mL})$. The combined organic layer was dried over $\mathrm{Na}_{2} \mathrm{SO}_{4}$ and concentrated under reduced pressure to afford crude mixture. Flash column chromatography (Hexane: $\mathrm{CH}_{2} \mathrm{Cl}_{2}=40: 60$ to Hexane:EtOAc $=80: 20$ ) afforded anomeric hemiacetal $\mathbf{S 2}$ as a white solid $(3.27 \mathrm{~g}, 13.4 \mathrm{mmol}, 61 \%)$ and recovered starting material $(1.53 \mathrm{~g}, 4.36 \mathrm{mmol}, 20 \%$ yield).

$\mathrm{R}_{f} 0.31$ (EtOAc:Hex $\left.=20: 80\right)$; m.p $65-66^{\circ} \mathrm{C}$.; $[\alpha]^{16}{ }_{\mathrm{D}}-84.53\left(\mathrm{c}=0.81, \mathrm{CH}_{2} \mathrm{Cl}_{2}\right) ;{ }^{1} \mathrm{H} \mathrm{NMR}\left(500 \mathrm{MHz}, \mathrm{CDCl}_{3}\right) \delta 5.82-$ $5.87\left(\mathrm{~m}, 1 \mathrm{H}_{\mathrm{a}} 1 \mathrm{H}_{\mathrm{b}}\right), 5.71-5.74\left(\mathrm{~m}, 1 \mathrm{H}_{\mathrm{a}} 1 \mathrm{H}_{\mathrm{b}}\right), 5.38\left(\mathrm{~d}, J=6.56 \mathrm{~Hz}, 1 \mathrm{H}_{\mathrm{b}}\right), 5.32\left(\right.$ brs, $\left.1 \mathrm{H}_{\mathrm{a}}\right), 3.95-3.97\left(\mathrm{~m}, 1 \mathrm{H}_{\mathrm{b}}\right), 3.78$, $3.87\left(\mathrm{~m}, 2 \mathrm{H}_{\mathrm{a}}\right), 3.56\left(\mathrm{qd}, J=6.22,8.04 \mathrm{~Hz}, 1 \mathrm{H}_{\mathrm{b}}\right), 2.90-3.14\left(\mathrm{~m}, 1 \mathrm{H}_{\mathrm{a}} 1 \mathrm{H}_{\mathrm{b}}\right), 1.30\left(\mathrm{~d}, J=6.22 \mathrm{~Hz}, 3 \mathrm{H}_{\mathrm{b}}\right), 1.25(\mathrm{~d}, J=$ $\left.6.10 \mathrm{~Hz}, 3 \mathrm{H}_{\mathrm{a}}\right), 0.90\left(\mathrm{~s}, 9 \mathrm{H}_{\mathrm{a}} 9 \mathrm{H}_{\mathrm{b}}\right), 0.098\left(\mathrm{~s}, 3 \mathrm{H}_{\mathrm{a}} 3 \mathrm{H}_{\mathrm{b}}\right), 0.087\left(\mathrm{~s}, 3 \mathrm{H}_{\mathrm{a}} 3 \mathrm{H}_{\mathrm{b}}\right)$.; ${ }^{13} \mathrm{C}$ NMR $\left(125 \mathrm{MHz}, \mathrm{CDCl}_{3}\right) \delta 135.1$, 134.5, 128. 9, 126.4, 92.4, 89.2, 75.1, 70.2, 69.9, 68.0, 25.9, 18.4, 18.2, -4.01, -4.48.; IR (NaCl) v 3361, 2988, 2955, 2932, 2901, 2858, 1462, 1408, 1381, $1361 \mathrm{~cm}^{-1}$; HRMS (ESI) m/z: $[\mathrm{M}+\mathrm{Na}]^{+}$Calcd for $\mathrm{C}_{12} \mathrm{H}_{24} \mathrm{O}_{3} \mathrm{SiNa}$ 267.1387; Found 267.1388.<smiles>CC(=O)OC1C=CC(O[18O]C(C)=O)[C@@H](C)O1</smiles>

3

(2S,5R,6S)-5-((tert-butyldimethylsilyl)oxy)-6-methyl-5,6-dihydro-2H-pyran-2-yl acetate (3) : To a solution of compound $\mathbf{S 2}(3.27 \mathrm{mg}, 13.4 \mathrm{mmol})$ in pyridine $(45 \mathrm{~mL}), \mathrm{Ac}_{2} \mathrm{O}(1.9 \mathrm{~mL}, 20.1 \mathrm{mmol})$ and DMAP $(6.5 \mathrm{mg}$, $0.053 \mathrm{mmol}$ ) was dropwisely added at $-15^{\circ} \mathrm{C}$ over $30 \mathrm{~min}$. After stirred at $-15^{\circ} \mathrm{C}$ for $1 \mathrm{~h}$. the reaction was quenched with distilled water then, extracted with $\mathrm{CH}_{2} \mathrm{Cl}_{2}(3 \times 50 \mathrm{~mL})$, dried over $\mathrm{Na}_{2} \mathrm{SO}_{4}$ and concentrated under reduced pressure. The crude mixture was purified by flash column chromatography (Hexane:Et $\left.\mathrm{Et}_{2} \mathrm{O}=95: 5\right)$ to afford $\alpha$ product 3 as white solid ( $3.33 \mathrm{~g}, 11.7 \mathrm{mmol}, 87 \%$ yield) and $\beta$-product ( $222 \mathrm{mg}, 0.78 \mathrm{mmol}, 5.8 \%$ yield) $\mathrm{R}_{f} 0.42$ (EtOAc:Hex = 10:90);m.p 71-72 ${ }^{\circ} \mathrm{C} . ;[\alpha]^{18}{ }_{\mathrm{D}}-64.83\left(\mathrm{c}=0.74, \mathrm{CH}_{2} \mathrm{Cl}_{2}\right) ;{ }^{1} \mathrm{H}$ NMR $\left(500 \mathrm{MHz}, \mathrm{CDCl}_{3}\right) \delta 6.21$ $(\mathrm{d}, J=10.15 \mathrm{~Hz}, 1 \mathrm{H}), 5.95(\mathrm{~d}, J=10.15 \mathrm{~Hz}, 1 \mathrm{H}), 5.67-5.70(\mathrm{~m}, 1 \mathrm{H}), 3.89-3.91(\mathrm{~m}, 1 \mathrm{H}), 3.75(\mathrm{qd}, J=6.20,8.75$ $\mathrm{Hz}, 1 \mathrm{H}), 2.07(\mathrm{~s}, 3 \mathrm{H}), 1.26(\mathrm{~d}, J=6.20 \mathrm{~Hz}, 3 \mathrm{H}), 0.90(\mathrm{~s}, 9 \mathrm{H}), 0.11(\mathrm{~s}, 3 \mathrm{H}), 0.093(\mathrm{~s}, 3 \mathrm{H}) . ;{ }^{13} \mathrm{C}$ NMR $(125 \mathrm{MHz}$, $\left.\mathrm{CDCl}_{3}\right) \delta 170.4,136.1,123.9,89.0,70.3,69.8,25.9,21.5,18.3,18.2,-4.03,-4.52 . ; \mathrm{IR}(\mathrm{NaCl}) \vee 3077,3048,2991$, 2930, 2857, 1743, 1464, 1371, $1320 \mathrm{~cm}^{-1}$; HRMS (ESI) m/z: [M + Na] ${ }^{+}$Calcd for $\mathrm{C}_{14} \mathrm{H}_{26} \mathrm{O}_{4} \mathrm{SiNa}$ 309.1493; Found 309.1494 .

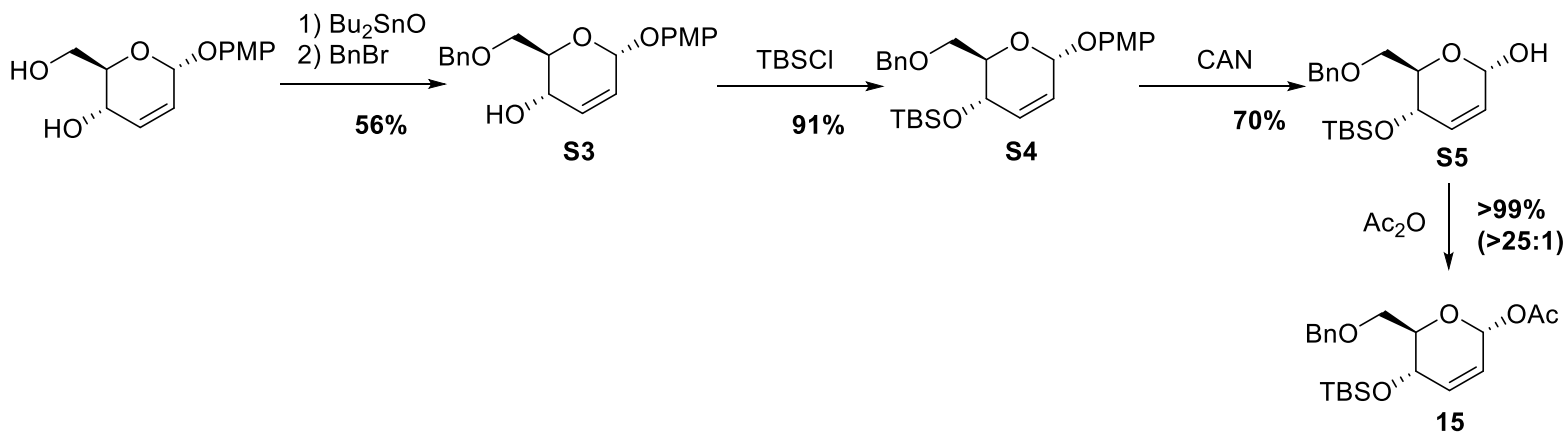

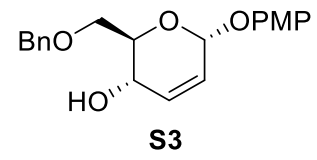


(2R,3S,6R)-2-((benzyloxy)methyl)-6-(4-methoxyphenoxy)-3,6-dihydro-2H-pyran-3-ol (S3) : To a solution of (2R,3S,6R)-2-(hydroxymethyl)-6-(4-methoxyphenoxy)-3,6-dihydro-2H-pyran-3-ol ${ }^{3}$ (5.2 $\left.\mathrm{g}, 20.6 \mathrm{mmol}\right)$ in toluene $(100 \mathrm{~mL})$ was added $\mathrm{Bu}_{2} \mathrm{SnO}(5.62 \mathrm{~g}, 22.7 \mathrm{mmol})$. The mixture was stirred at $135^{\circ} \mathrm{C}$ in a Dean-Stark overnight. The reaction mixture was cooled, $\mathrm{Bu}_{4} \mathrm{NI}(7.61 \mathrm{~g}, 20.6 \mathrm{mmol})$ and benzyl bromide $(2.94 \mathrm{~mL}, 24.7 \mathrm{mmol})$ were added, and the mixture was stirred at $110^{\circ} \mathrm{C}$ for $8 \mathrm{~h}$. The mixture was cooled and quenched with sat NaHCO3, extracted with EtOAc ( 4 x $50 \mathrm{~mL})$, filtered by a pad of celite (rinse; EtOAc) and concentrated under reduced pressure. The crude mixture was purified by flash column chromatography (Hexane:EtOAc $=70: 30$ ) to afford product $\mathbf{S 3}$ as a colorless oil (3.95 g, $11.5 \mathrm{mmol}, 56 \%$ yield) and recovered starting diol (938 $\mathrm{mg}, 3.72 \mathrm{mmol}, 18 \%$ yield)

$\mathrm{R}_{f} 0.42$ (EtOAc:Hex = 40:60); $[\alpha]^{15} \mathrm{D}+112.17\left(\mathrm{c}=1.13 \mathrm{CH}_{2} \mathrm{Cl}_{2}\right) ;{ }^{1} \mathrm{H}$ NMR $\left(300 \mathrm{MHz}, \mathrm{CDCl}_{3}\right) \delta$ 7.27-7.38 (m, $5 \mathrm{H}), 7.01-7.06(\mathrm{~m}, 2 \mathrm{H}), 6.78-6.83(\mathrm{~m}, 2 \mathrm{H}), 6.05-6.10(\mathrm{~m}, 1 \mathrm{H}), 5.88-5.93(\mathrm{~m}, 1 \mathrm{H}), 5.52-5.53(\mathrm{~m}, 1 \mathrm{H}), 4.53-4.63$ $(\mathrm{m}, 2 \mathrm{H}), 4.28-4.34(\mathrm{~m}, 1 \mathrm{H}), 3.97-4.04(\mathrm{~m}, 1 \mathrm{H}), 3.67-3.80(\mathrm{~m}, 5 \mathrm{H}), 2.49-2.50(\mathrm{~m}, 1 \mathrm{H}) . ;{ }^{13} \mathrm{C} \mathrm{NMR}\left(75 \mathrm{MHz}, \mathrm{CDCl}_{3}\right)$ $\delta 155.2,151.5,137.8,133.9,128.7,128.1,128.0,125.6,118.7,114.7,94.1,73.9,71.0,70.5,66.0,55.8 . ; \operatorname{IR}(\mathrm{NaCl})$ $v$ 3432, 3033, 2908, 2835, 1506, 1454, 1389, 1292, 1243, 1215, 1183, $1107 \mathrm{~cm}^{-1}$; HRMS (ESI) m/z: [M + Na] $]^{+}$ Calcd for $\mathrm{C}_{20} \mathrm{H}_{22} \mathrm{O}_{5} \mathrm{Na} 365.1359$; Found 365.1362.

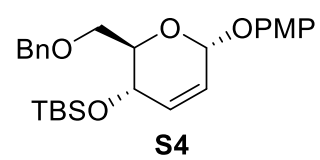

(((2R,3S,6R)-2-((benzyloxy)methyl)-6-(4-methoxyphenoxy)-3,6-dihydro-2H-pyran-3-yl)oxy)(tert-

butyl)dimethylsilane (S4) : As same procedure as described for S1 using compound S3 (3.94 g, $11.5 \mathrm{mmol})$, The crude oil was purified with flashcolumn chromatography (Hexane: $\mathrm{Et}_{2} \mathrm{O}=95: 5$ ) to give desired product $\mathbf{S 4}$ (4.80 g, $10.5 \mathrm{mmol}, 91 \%$ yield) as a colorless oil.

$\mathrm{R}_{f} 0.42$ (EtOAc:Hex = 10:90); $[\alpha]^{15}{ }_{\mathrm{D}}+135.74\left(\mathrm{c}=1.29 \mathrm{CH}_{2} \mathrm{Cl}_{2}\right) ;{ }^{1} \mathrm{H}$ NMR $\left(300 \mathrm{MHz}, \mathrm{CDCl}_{3}\right) \delta$ 7.26-7.33 (m, $5 \mathrm{H}), 7.07-7.10(\mathrm{~m}, 2 \mathrm{H}), 6.78-6.81(\mathrm{~m}, 2 \mathrm{H}), 6.00-6.02(\mathrm{~m}, 2 \mathrm{H}), 5.86(\mathrm{ddd}, J=2.08,2.36,10.18 \mathrm{~Hz}, 1 \mathrm{H}), 5.55-5.56$ $(\mathrm{m}, 1 \mathrm{H}), 4.56(\mathrm{~s}, 2 \mathrm{H}), 4.36-4.41(\mathrm{~m}, 1 \mathrm{H}), 3.99-4.05(\mathrm{~m}, 1 \mathrm{H}), 3.77(\mathrm{~s}, 3 \mathrm{H}), 3.70(\mathrm{~d}, J=3.41 \mathrm{~Hz}, 1 \mathrm{H}), 0.89(\mathrm{~s}, 9 \mathrm{H})$, $0.12(\mathrm{~s}, 3 \mathrm{H}), 0.070(\mathrm{~s}, 3 \mathrm{H}) . ;{ }^{13} \mathrm{C}$ NMR $\left(75 \mathrm{MHz}, \mathrm{CDCl}_{3}\right) \delta 155.2,151.7,138.4,135.4,128.5,127.9,127.7,125.1$, 119.0, 114.7, 94.6, 73.5, 72.0, 69.2, 64.1, 55.8, 25.9, 18.1, -3.99, -4.70.; IR (NaCl) v 3047, 3000, 2954, 2930, 2900 , 2857, 1506, 1466, 1389, 1362, 1311, $1250 \mathrm{~cm}^{-1}$; HRMS (ESI) m/z: [M + Na $]^{+}$Calcd for $\mathrm{C}_{26} \mathrm{H}_{36} \mathrm{O}_{5} \mathrm{SiNa} 479.2224$; Found 479.2220 .

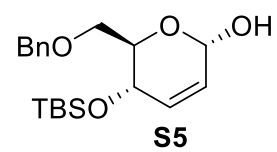

(2S,5S,6R)-6-((benzyloxy)methyl)-5-((tert-butyldimethylsilyl)oxy)-5,6-dihydro-2H-pyran-2-ol (S5) : As same procedure as described for $\mathbf{S 2}$ using compound $\mathbf{S 4}(4.80 \mathrm{~g}, 10.5 \mathrm{mmol})$, The crude oil was purified with flashcolumn chromatography (Hexane: $\mathrm{CH}_{2} \mathrm{Cl}_{2}=40: 60$ to Hexane:EtOAc $=80: 20$ ) afforded hemiacetal $\mathbf{S 5}$ as a white solid (2.59 g, $7.40 \mathrm{mmol}, 70 \%$ yield) and recovered starting material (1.10 g, $2.42 \mathrm{mmol}, 23 \%$ yield).

$\mathrm{R}_{f} 0.25$ (EtOAc:Hex = 20:80); m.p 76-77 ${ }^{\circ} \mathrm{C}$; $[\alpha]^{16}{ }_{\mathrm{D}}+59.86\left(\mathrm{c}=1.10 \mathrm{CH}_{2} \mathrm{Cl}_{2}\right) ;{ }^{1} \mathrm{H}$ NMR $\left(500 \mathrm{MHz}, \mathrm{CDCl}_{3}\right) \delta$ 7.25-7.36 (m, $5 \mathrm{H}), 5.86(\mathrm{~d}, J=10.28 \mathrm{~Hz}, 1 \mathrm{H}), 5.71-5.75(\mathrm{~m}, 1 \mathrm{H}), 5.42(\mathrm{brs}, 1 \mathrm{H}), 4.62(\mathrm{~d}, J=12.07 \mathrm{~Hz}, 1 \mathrm{H}), 4.56$ $(\mathrm{d}, J=12.07 \mathrm{~Hz}, 1 \mathrm{H}), 4.22-4.24(\mathrm{~m}, 1 \mathrm{H}), 3.93(\mathrm{ddd}, J=2.07,5.19,8.77 \mathrm{~Hz}, 1 \mathrm{H}), 3.68(\mathrm{dd}, J=2.07,10.37 \mathrm{~Hz}$, $1 \mathrm{H}), 3.62(\mathrm{dd}, J=5.19,10.37 \mathrm{~Hz}, 1 \mathrm{H}), 3.36(\mathrm{~d}, J=4.71 \mathrm{~Hz}, 1 \mathrm{H}), 0.85(\mathrm{~s}, 9 \mathrm{H}), 0.074(\mathrm{~s}, 3 \mathrm{H}), 0.010(\mathrm{~s}, 3 \mathrm{H}) . ;{ }^{13} \mathrm{C}$ NMR $\left(125 \mathrm{MHz}, \mathrm{CDCl}_{3}\right) \delta 138.1,134.5,128.5,128.1,127.8,126.5,89.2,73.6,71.2,69.3,64.3,25.9,18.1,-4.03$, -4.73.; IR (NaCl) v 3418, 3033, 2954, 2929, 2895, 2858, 1496, 1470, 1408, 1377, 1362, $1255 \mathrm{~cm}^{-1}$; HRMS (ESI) $\mathrm{m} / \mathrm{z}:[\mathrm{M}+\mathrm{Na}]^{+}$Calcd for $\mathrm{C}_{19} \mathrm{H}_{30} \mathrm{O}_{4} \mathrm{SiNa} 373.1806$; Found 373.1812 .<smiles>CC(=O)O[C@@H]1C=C[C@@H](O[SnH3])[C@H](COc2ccccc2)O1</smiles>

(2R,5S,6R)-6-((benzyloxy)methyl)-5-((tert-butyldimethylsilyl)oxy)-5,6-dihydro-2H-pyran-2-yl acetate (15) : 
As same procedure as described for 3 using compound S5 (2.57 g, $7.34 \mathrm{mmol})$, The crude oil was purified with flashcolumn chromatography (Hexane: $\left.\mathrm{Et}_{2} \mathrm{O}=95: 5\right)$ to afford $\alpha$-product 15 as a colorless oil (2.92 $\mathrm{g}, 7.35 \mathrm{mmol}$, $>99 \%$ yield).

$\mathrm{R}_{f} 0.26$ (EtOAc:Hex = 10:90); $[\alpha]^{16}{ }_{\mathrm{D}}+34.70\left(\mathrm{c}=1.00 \mathrm{CH}_{2} \mathrm{Cl}_{2}\right) ;{ }^{1} \mathrm{H}$ NMR $\left(300 \mathrm{MHz}, \mathrm{CDCl}_{3}\right) \delta 7.25-7.36(\mathrm{~m}, 5 \mathrm{H})$, 6.28-6.29 (m, 1H), $5.97(\mathrm{~d}, J=10.14 \mathrm{~Hz}, 1 \mathrm{H}), 5.72(\mathrm{ddd}, J=1.89,2.93,10.14 \mathrm{~Hz}, 1 \mathrm{H}), 4.62(\mathrm{~d}, J=12.06 \mathrm{~Hz}$, $1 \mathrm{H}), 4.55(\mathrm{~d}, J=12.06 \mathrm{~Hz}, 1 \mathrm{H}), 4.33-4.38(\mathrm{~m}, 1 \mathrm{H}), 3.81-3.86(\mathrm{~m}, 1 \mathrm{H}), 3.63-3.73(\mathrm{~m}, 2 \mathrm{H}), 2.07(\mathrm{~s}, 3 \mathrm{H}), 0.87(\mathrm{~s}$, 9H), $0.094(\mathrm{~s}, 3 \mathrm{H}), 0.039$ (s, 3H).; ${ }^{13} \mathrm{C} \mathrm{NMR}\left(75 \mathrm{MHz}, \mathrm{CDCl}_{3}\right) \delta 170.3,138.3,135.7,128.5,128.0,127.8,123.8$, 89.2, 73.9, 73.8, 68.8, 63.7, 25.9, 21.5, 18.1, -4.06, -4.76.; IR (NaCl) v 3031, 2955, 2930, 2896, 2858, 1749, 1497, 1471, 1369, 1313, 1231, $1193 \mathrm{~cm}^{-1}$; HRMS (ESI) m/z: $[\mathrm{M}+\mathrm{Na}]^{+}$Calcd for $\mathrm{C}_{21} \mathrm{H}_{32} \mathrm{O}_{5} \mathrm{SiNa} 415.1911$; Found 415.1912.

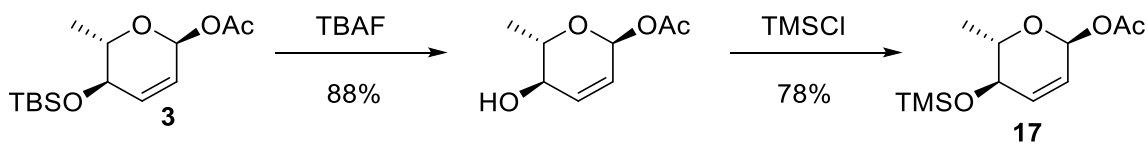<smiles></smiles>

(2S,5R,6S)-6-methyl-5-((trimethylsilyl)oxy)-5,6-dihydro-2H-pyran-2-yl acetate (17) : To a solution of compound 3 (286 mg, $1.0 \mathrm{mmol})$ in THF $(5 \mathrm{~mL})$, TBAF $(1.1 \mathrm{~mL}, 1.1 \mathrm{mmol})$ was added. The reaction mixture was stirred at room temperature for $10 \mathrm{~min}$. After complete conversion, mixture was concentrated under reduced pressure. The crude mixture was purified by flash column chromatography (Hexane:EtOAc $=60: 40$ ) to afford alcohol as a colorless oil $(151 \mathrm{mg}, 0.88 \mathrm{mmol}, 88 \%)$. A solution of the isolated alcohol (148 $\mathrm{mg}, 0.86 \mathrm{mmol}) \mathrm{in}$ DMF $(2.9 \mathrm{~mL})$ was treated with imidazole $(176 \mathrm{mg}, 2.58 \mathrm{mmol})$ and $\mathrm{TMSCl}(187 \mathrm{mg}, 1.72 \mathrm{mmol})$ at $0^{\circ} \mathrm{C}$. The reaction mixture was stirred at room temperature for $0.5 \mathrm{~h}$. The reaction mixture was quenched with $\mathrm{H}_{2} \mathrm{O}(30 \mathrm{~mL})$. The organic residue was extracted with $\mathrm{Et}_{2} \mathrm{O}(4 \times 50 \mathrm{~mL})$, wahsed with brine $(3 \times 50 \mathrm{~mL})$ dried over $\mathrm{Na}_{2} \mathrm{SO}_{4}$ and concentrated under reduced pressure. The crude mixture was purified by flash column chromatography (Hexane: $\mathrm{Et}_{2} \mathrm{O}=95: 5$ ) to afford product 17 as colorless oil (164 mg, $0.67 \mathrm{mmol}, 78 \%$ yield).

$\mathrm{R}_{f} 0.37$ (EtOAc:Hex = 10:90); $\left[\alpha{ }^{16}{ }_{\mathrm{D}}-56.28\left(\mathrm{c}=0.73, \mathrm{CH}_{2} \mathrm{Cl}_{2}\right) ;{ }^{1} \mathrm{H}\right.$ NMR $\left(300 \mathrm{MHz}, \mathrm{CDCl}_{3}\right) \delta 6.20-6.21(\mathrm{~m}, 1 \mathrm{H})$. $5.93(\mathrm{~d}, J=10.16 \mathrm{~Hz}, 1 \mathrm{H}), 5.70(\mathrm{ddd}, J=2.06,2.83,10.16 \mathrm{~Hz}, 1 \mathrm{H}), 3.88-3.92(\mathrm{~m}, 1 \mathrm{H}), 3.76(\mathrm{dq}, J=6.13,8.73$ $\mathrm{Hz}, 1 \mathrm{H}), 2.06(\mathrm{~s}, 3 \mathrm{H}), 1.25(\mathrm{~d}, J=6.13 \mathrm{~Hz}, 3 \mathrm{H}), 0.16(\mathrm{~s}, 9 \mathrm{H}), ;{ }^{13} \mathrm{C} \mathrm{NMR}\left(75 \mathrm{MHz}, \mathrm{CDCl}_{3}\right) \delta 170.4,135.8,124.1$, 88.9, 70.1, 69.5, 21.5, 18.2, 0.41.; IR (NaCl) v 3051, 2959, 2904, 2846, 1749, 1453, 1398, 1371, $1310 \mathrm{~cm}^{-1}$; HRMS (ESI) $\mathrm{m} / \mathrm{z}:[\mathrm{M}+\mathrm{Na}]^{+}$Calcd for $\mathrm{C}_{11} \mathrm{H}_{20} \mathrm{O}_{4} \mathrm{SiNa} 267.1023$; Found 267.1023.
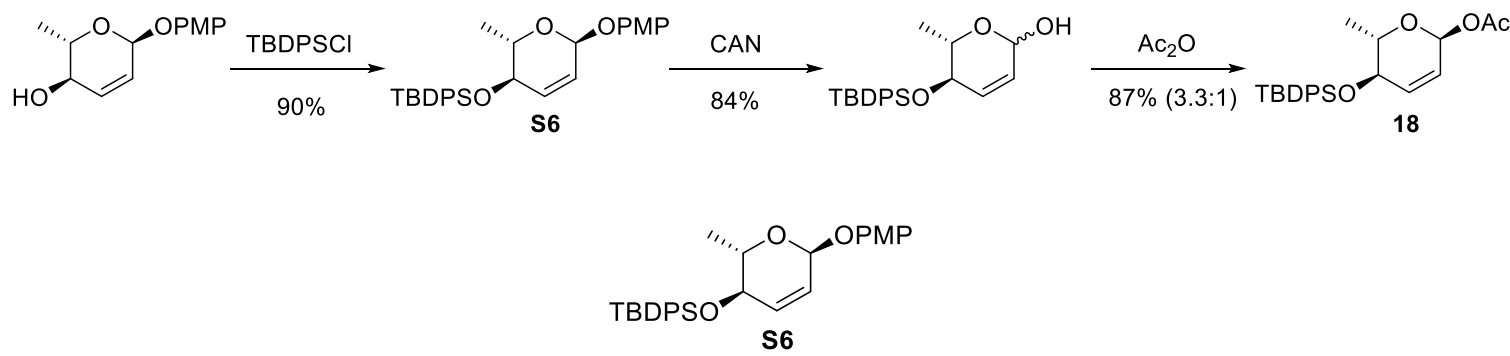

tert-butyl(((2S,3R,6S)-6-(4-methoxyphenoxy)-2-methyl-3,6-dihydro-2H-pyran-3-yl)oxy)diphenylsilane (S6) : A solution of (2S,3R,6S)-6-(4-methoxyphenoxy)-2-methyl-3,6-dihydro-2H-pyran-3-ol ${ }^{2}$ (472 mg, $2 \mathrm{mmol}$ ) in DMF $(6.6 \mathrm{~mL})$ was treated with imidazole $(409 \mathrm{mg}, 6 \mathrm{mmol})$ and TBDPSCl $(1.10 \mathrm{~g}, 4 \mathrm{mmol})$ at $0^{\circ} \mathrm{C}$. The reaction mixture was stirred at $40^{\circ} \mathrm{C}$ for $15 \mathrm{~h}$. The reaction mixture was quenched with $\mathrm{H}_{2} \mathrm{O}(30 \mathrm{~mL})$. The organic residue was extracted with $\mathrm{Et}_{2} \mathrm{O}(4 \times 50 \mathrm{~mL})$, wahsed with brine $(3 \times 50 \mathrm{~mL})$ dried over $\mathrm{Na}_{2} \mathrm{SO}_{4}$ and concentrated under reduced pressure. The crude mixture was purified by flash column chromatography $\left(\mathrm{Hexane}_{2} \mathrm{Et}_{2} \mathrm{O}=95: 5\right)$ to afford product $\mathbf{S 6}$ as colorless oil $(855 \mathrm{mg}, 1.8 \mathrm{mmol}, 90 \%$ yield).

$\mathrm{R}_{f} 0.45$ (EtOAc:Hex = 10:90); $[\alpha]^{16}{ }_{\mathrm{D}}-107.51\left(\mathrm{c}=1.28, \mathrm{CH}_{2} \mathrm{Cl}_{2}\right) ;{ }^{1} \mathrm{H} \mathrm{NMR}\left(300 \mathrm{MHz}, \mathrm{CDCl}_{3}\right) \delta$ 7.69-7.73 (m, $4 \mathrm{H}), 7.37-7.49(\mathrm{~m}, 6 \mathrm{H}), 7.00-7.05(\mathrm{~m}, 2 \mathrm{H}), 6.82-6.86(\mathrm{~m}, 2 \mathrm{H}), 5.90(\mathrm{~d}, J=10.19 \mathrm{~Hz}, 1 \mathrm{H}), 5.69-5.74(\mathrm{~m}, 1 \mathrm{H})$, 5.39-5.40 (m, 1H), 3.99-4.10 (m, 2H), $3.78(\mathrm{~s}, 3 \mathrm{H}), 1.20(\mathrm{~d}, J=5.85 \mathrm{~Hz}, 3 \mathrm{H}), 1.09(\mathrm{~s}, 9 \mathrm{H})$; ${ }^{13} \mathrm{C} \mathrm{NMR}(75 \mathrm{MHz}$, $\left.\mathrm{CDCl}_{3}\right) \delta 155.0,151.8,136.21,136.16,135.1,134.1,133.4,130.1,130.0,128.0,127.8,125.0,118.6,114.7,94.4$, 
71.2, 68.8, 55.8, 27.2, 19.6, 18.5.; IR (NaCl) v 3071, 3049, 2933, 2899, 2858, 1506, 1467, 1428, 1396, $1362 \mathrm{~cm}^{-}$ 1; HRMS (ESI) m/z: [M + Na ${ }^{+}$Calcd for $\mathrm{C}_{29} \mathrm{H}_{34} \mathrm{O}_{4} \mathrm{SiNa}$ 497.2119; Found 497.2117.

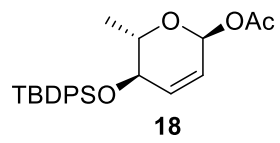

(2S,5R,6S)-5-((tert-butyldiphenylsilyl)oxy)-6-methyl-5,6-dihydro-2H-pyran-2-yl acetate (18) : As same procedure as described for S2 using compound S6 $(827 \mathrm{mg}, 1.74 \mathrm{mmol})$, The crude oil was purified with flashcolumn chromatography (Hexane:EtOAc $=90: 10)$ to give anomeric mixture $(538 \mathrm{mg}, 1.46 \mathrm{mmol}, 84 \%)$ as a colorless oil. To a solution of isolated product in pyridine $(5 \mathrm{~mL}), \mathrm{Ac}_{2} \mathrm{O}(0.21 \mathrm{~mL}, 2.19 \mathrm{mmol})$ and DMAP (17.8 $\mathrm{mg}, 0.146 \mathrm{mmol}$ ) was dropwisely added at $-15^{\circ} \mathrm{C}$ over $30 \mathrm{~min}$. After stirred at $-15^{\circ} \mathrm{C}$ for $1 \mathrm{~h}$. The reaction was quenched with $\mathrm{H}_{2} \mathrm{O}$, extracted with $\mathrm{CH}_{2} \mathrm{Cl}_{2}(3 \times 50 \mathrm{~mL})$, dried over $\mathrm{Na}_{2} \mathrm{SO}_{4}$ and concentrated under reduced pressure. The crude mixture was purified by flash column chromatography (Hexane:Et $2 \mathrm{O}=95: 5$ ) to afford $\alpha$ product 18 as a colorless oil (400 mg, $0.975 \mathrm{mmol}, 67 \%$ yield) and $\beta$-product (121 mg, $0.30 \mathrm{mmol}, 20 \%$ yield) $\mathrm{R}_{f} 0.41$ (EtOAc:Hex = 10:90); $[\alpha]{ }^{17} \mathrm{D}-61.75\left(\mathrm{c}=1.06, \mathrm{CH}_{2} \mathrm{Cl}_{2}\right) ;{ }^{1} \mathrm{H}$ NMR $\left(300 \mathrm{MHz}, \mathrm{CDCl}_{3}\right) \delta 7.67-7.70(\mathrm{~m}, 4 \mathrm{H})$, 7.36-7.48 (m, 6H), 6.12-6.13 (m, 1H), $5.88(\mathrm{~d}, J=10.27 \mathrm{~Hz}, 1 \mathrm{H}), 5.54-5.59(\mathrm{~m}, 1 \mathrm{H}), 3.98-4.01(\mathrm{~m}, 1 \mathrm{H}), 3.89(\mathrm{qd}$, $J=6.09,8.74 \mathrm{~Hz}, 1 \mathrm{H}), 2.10(\mathrm{~s}, 3 \mathrm{H}), 1.21(\mathrm{~d}, J=6.09 \mathrm{~Hz}, 3 \mathrm{H}), 1.08(\mathrm{~s}, 9 \mathrm{H}) . ;{ }^{13} \mathrm{C} \mathrm{NMR}\left(75 \mathrm{MHz}, \mathrm{CDCl}_{3}\right) \delta 170.4$, 136.2, 136.1, 135.5, 134.0, 133.2, 130.2, 130.1, 128.0, 127.9, 123.7, 88.9, 70.8, 70.3, 27.2, 21.5, 19.6, 18.4.; IR $(\mathrm{NaCl}) \vee 3072,3051,2961,2934,2859,1749,1589,1472,1428,1396 \mathrm{~cm}^{-1} ; \mathrm{HRMS}(\mathrm{ESI}) \mathrm{m} / \mathrm{z}:[\mathrm{M}+\mathrm{Na}]^{+} \mathrm{Calcd}$ for $\mathrm{C}_{24} \mathrm{H}_{30} \mathrm{O}_{4} \mathrm{SiNa} 433.1806$; Found 433.1808.

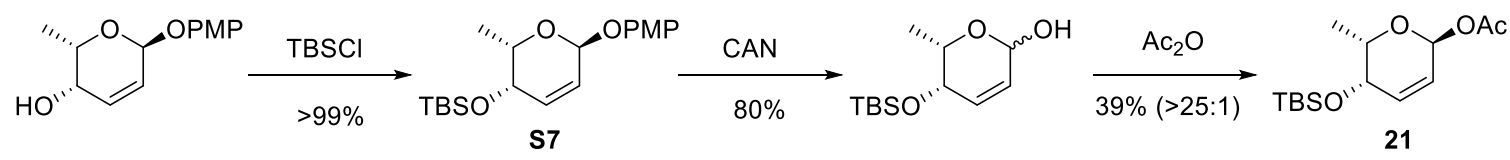<smiles>C[C@@H]1OC(O[Na])C=C[C@@H]1O[Na]</smiles>

tert-butyl(((2S,3S,6S)-6-(4-methoxyphenoxy)-2-methyl-3,6-dihydro-2H-pyran-3-yl)oxy)dimethylsilane (S7) : As same procedure as described for S1 using (2S,3S,6S)-6-(4-methoxyphenoxy)-2-methyl-3,6-dihydro-2Hpyran-3-ol ${ }^{2}$ (916 mg, $\left.3.88 \mathrm{mmol}\right)$, The crude oil was purified with flashcolumn chromatography $\left(\mathrm{Hexane}_{2} \mathrm{Et}_{2} \mathrm{O}=\right.$ 95:5) to give desired product $\mathbf{S} 7(1.36 \mathrm{~g}, 3.88 \mathrm{mmol},>99 \%$ yield $)$ as a colorless oil.

$\mathrm{R}_{f} 0.44$ (EtOAc:Hex = 10:90); $[\alpha]^{18}{ }_{\mathrm{D}}+50.32\left(\mathrm{c}=0.85, \mathrm{CH}_{2} \mathrm{Cl}_{2}\right) ;{ }^{1} \mathrm{H}$ NMR $\left(300 \mathrm{MHz}, \mathrm{CDCl}_{3}\right) \delta 7.03-7.08(\mathrm{~m}, 2 \mathrm{H})$, $6.80-6.85(\mathrm{~m}, 2 \mathrm{H}), 6.14(\mathrm{dd}, J=5.34,9.99 \mathrm{~Hz}, 1 \mathrm{H}), 6.00(\mathrm{dd}, J=3.03,9.99 \mathrm{~Hz}, 1 \mathrm{H}), 5.59(\mathrm{~d}, J=3.03 \mathrm{~Hz}, 1 \mathrm{H})$, 4.20-4.27 (m, 1H), 3.75-3.77 (m, 4H), $1.24(\mathrm{~d}, J=6.58 \mathrm{~Hz}, 3 \mathrm{H}), 0.92(\mathrm{~s}, 9 \mathrm{H}), 0.11(\mathrm{~s}, 3 \mathrm{H}), 0.10(\mathrm{~s}, 3 \mathrm{H}) . ;{ }^{13} \mathrm{C}$ NMR $\left(75 \mathrm{MHz}, \mathrm{CDCl}_{3}\right) \delta 155.0,151.8,131.0,127.0,118.5,114.7,94.3,67.8,64.4,55.9,26.1,18.5,16.7,-4.00$, -4.44.; IR (NaCl) v 3046, 2955, 2932, 2895, 2835, 1506, 1466, 1443, 1440, $1378 \mathrm{~cm}^{-1}$; HRMS (ESI) m/z: [M + $\mathrm{Na}]^{+}$Calcd for $\mathrm{C}_{19} \mathrm{H}_{30} \mathrm{O}_{4} \mathrm{SiNa} 373.1806$; Found 373.1807.<smiles>[Z17]C[C@H]1C=C[C@@H](OC(C)=O)O[C@H]1C</smiles>

(2S,5S,6S)-5-((tert-butyldimethylsilyl)oxy)-6-methyl-5,6-dihydro-2H-pyran-2-yl acetate (21) : As same procedure as described for S2 using compound S7 (1.43 g, $4.07 \mathrm{mmol})$, The crude oil was purified with flashcolumn chromatography (Hexane:EtOAc $=80: 20)$ to give anomeric mixture $(796 \mathrm{mg}, 3.26 \mathrm{mmol}, 80 \%)$. To a solution of isolated product $(793 \mathrm{mg}, 3.25 \mathrm{mmol})$ in pyridine $(10 \mathrm{~mL}), \mathrm{Ac}_{2} \mathrm{O}(0.46 \mathrm{~mL}, 4.88 \mathrm{mmol})$ and DMAP (39.7 $\mathrm{mg}, 0.325 \mathrm{mmol}$ ) was dropwisely added at $-15^{\circ} \mathrm{C}$ over $30 \mathrm{~min}$. After stirred at $-15^{\circ} \mathrm{C}$ for $1 \mathrm{~h}$. The reaction was quenched with $\mathrm{H}_{2} \mathrm{O}$, extracted with $\mathrm{CH}_{2} \mathrm{Cl}_{2}(3 \times 50 \mathrm{~mL})$, dried over $\mathrm{Na}_{2} \mathrm{SO}_{4}$ and concentrated under reduced pressure. The crude mixture was purified by flash column chromatography (Hexane:Et ${ }_{2} \mathrm{O}=95: 5$ ) to afford $\alpha$ product 21 as a colorless oil (367 $\mathrm{mg}, 1.28 \mathrm{mmol}, 39 \%$ yield).

$\mathrm{R}_{f} 0.44$ (EtOAc:Hex = 10:90); $[\alpha]^{18}{ }_{\mathrm{D}}+198.74\left(\mathrm{c}=0.72, \mathrm{CH}_{2} \mathrm{Cl}_{2}\right) ;{ }^{1} \mathrm{H}$ NMR $\left(500 \mathrm{MHz}, \mathrm{CDCl}_{3}\right) \delta 6.31(\mathrm{~d}, J=3.09$ $\mathrm{Hz}, 1 \mathrm{H}), 6.13(\mathrm{dd}, J=5.37,9.86 \mathrm{~Hz}, 1 \mathrm{H}), 5.87(\mathrm{dd}, J=3.24,9.86 \mathrm{~Hz}, 1 \mathrm{H}), 4.07(\mathrm{dq}, J=2.55,6.52 \mathrm{~Hz}, 1 \mathrm{H}), 3.73$ 
$(\mathrm{dd}, J=2.55,5.37 \mathrm{~Hz}, 1 \mathrm{H}), 2.07(\mathrm{~s}, 3 \mathrm{H}), 1.25(\mathrm{~d}, J=6.57 \mathrm{~Hz}, 3 \mathrm{H}), 0.90(\mathrm{~s}, 9 \mathrm{H}), 0.086(\mathrm{~s}, 3 \mathrm{H}), 0.078(\mathrm{~s}, 3 \mathrm{H}) . ;{ }^{13} \mathrm{C}$ NMR $\left(125 \mathrm{MHz}, \mathrm{CDCl}_{3}\right) \delta 170.3,131.3,125.8,89.4,69.5,64.1,26.0,21.5,18.5,16.7,-3.92,-4.47 . ; \mathrm{IR}(\mathrm{NaCl}) v$ 3049, 2957, 2932, 2893, 2858, 1746, 1507, 1471, 1400, $1369 \mathrm{~cm}^{-1} ; \mathrm{HRMS}(\mathrm{ESI}) \mathrm{m} / \mathrm{z}:[\mathrm{M}+\mathrm{Na}]^{+}$Calcd for $\mathrm{C}_{14} \mathrm{H}_{26} \mathrm{O}_{4} \mathrm{SiNa} 309.1493$; Found 309.1497.
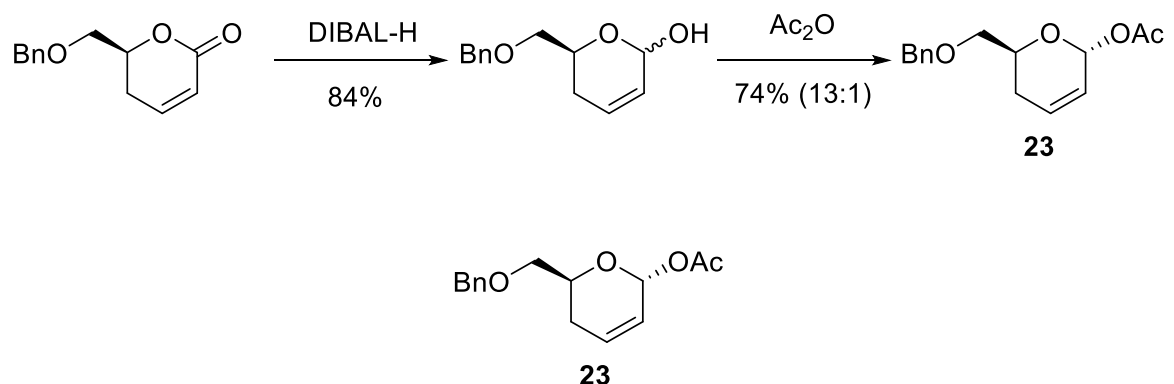

23

(2R,6S)-6-((benzyloxy)methyl)-5,6-dihydro-2H-pyran-2-yl acetate (23) : To a solution of (S)-6((benzyloxy)methyl)-5,6-dihydro-2H-pyran-2-one ${ }^{4}$ (1.54 g, $\left.7.0 \mathrm{mmol}\right)$ in $\mathrm{CH}_{2} \mathrm{Cl}_{2}(70 \mathrm{~mL})$, added DIBAL-H (10.5 $\mathrm{mL}, 10.5 \mathrm{mmol}, 1 \mathrm{M}$ in toluene) at $-78^{\circ} \mathrm{C}$ dropwisely. After stirred at same temperature for $20 \mathrm{~min}$, the reaction mixture was quenched with $\mathrm{MeOH}$ at $-78 \mathrm{oC}$, then allowed to warm at room temperature. The mixture was extracted with $\mathrm{Et}_{2} \mathrm{O}(6 \times 100 \mathrm{~mL})$, dried over $\mathrm{Na}_{2} \mathrm{SO}_{4}$ and concentrated under reduced pressure. The crude mixture was purified by flash column chromatography (Hexane:EtOAc $=80: 20)$ to hemiacetal $(1.31 \mathrm{~g}, 5.9 \mathrm{mmol}, 84 \%)$. To a solution of isolated product $(600 \mathrm{mg}, 3.0 \mathrm{mmol})$ in pyridine $(15 \mathrm{~mL}), \mathrm{Ac}_{2} \mathrm{O}(0.43 \mathrm{~mL}, 4.5 \mathrm{mmol})$ and DMAP (36.6 mg, $0.30 \mathrm{mmol}$ ) was dropwisely added at $-15^{\circ} \mathrm{C}$ over $30 \mathrm{~min}$. After stirred at $-15^{\circ} \mathrm{C}$ for $1 \mathrm{~h}$. The reaction was quenched with $\mathrm{H}_{2} \mathrm{O}$, extracted with $\mathrm{CH}_{2} \mathrm{Cl}_{2}(3 \times 50 \mathrm{~mL})$, dried over $\mathrm{Na}_{2} \mathrm{SO}_{4}$ and concentrated under reduced pressure. The crude mixture was purified by flash column chromatography (Hexane:Et $2 \mathrm{O}=95: 5$ ) to afford $\alpha$ product 23 as a colorless oil (367 $\mathrm{mg}, 1.28 \mathrm{mmol}, 74 \%$ yield, $\alpha / \beta=13 / 1)$.

$\mathrm{R}_{f} 0.54$ (EtOAc:Hex = 20:80); $[\alpha]^{18} \mathrm{D}-94.34\left(\mathrm{c}=1.49, \mathrm{CH}_{2} \mathrm{Cl}_{2}\right) ;{ }^{1} \mathrm{H} \mathrm{NMR}\left(300 \mathrm{MHz}, \mathrm{CDCl}_{3}\right) \delta 7.26-7.38(\mathrm{~m}, 5 \mathrm{H})$, 6.31-6.32 (m, 1H), 6.12-6.17 (m, 1H), 5.74-5.80 (m, 1H), $4.59(\mathrm{~s}, 2 \mathrm{H}), 4.09-4.17(\mathrm{~m}, 1 \mathrm{H}), 3.52-3.62(\mathrm{~m}, 2 \mathrm{H})$, 2.16-2.29 (m, 1H), 2.00-2.07 (m, 4H).; $\left.{ }^{13} \mathrm{C} \mathrm{NMR} \mathrm{(75} \mathrm{MHz,} \mathrm{CDCl}_{3}\right) \delta$ 170.3, 138.3, 129.9, 128.6, 127.9, 127.8, 123.8, 89.6, 73.7, 72.3, 68.5, 26.8, 21.5.; IR (NaCl) v 3038, 3032, 2911, 2864, 1735, 1497, 1453, 1427, 1370, $1322 \mathrm{~cm}^{-1}$; HRMS (ESI) m/z: [M + Na] ${ }^{+}$Calcd for $\mathrm{C}_{15} \mathrm{H}_{18} \mathrm{O}_{4} \mathrm{Na} 285.1097$; Found 285.1099.

\section{Stereoselective Synthesis of $C$-Glycoside 2a, 4-14, 16, 22, 24}<smiles>C[C@H]1O[C@H]([C@H](O)c2ccccc2)C=C[C@H]1OC(C)(C)C</smiles>

(S)-((2S,5R,6S)-5-((tert-butyldimethylsilyl)oxy)-6-methyl-5,6-dihydro-2H-pyran-2-yl)(phenyl)methanol (2a) : Following the general procedure using benzaldehyde $(20.5 \mu \mathrm{L}, 0.20 \mathrm{mmol})$ and acetate $\mathbf{3}(68.6 \mathrm{mg}, 0.24$ mmol), Flash column chromatography (EtOAc:Hexane $=5: 95)$ afforded the compound $\mathbf{2 a}$ as a colorless oil (52.8 $\mathrm{mg}, 0.158 \mathrm{mmol}, 79 \%$ yield). The diastereomer ratio (17:1) was determined by Crude NMR.

$\mathrm{R}_{f} 0.40$ (EtOAc:Hex = 10:90); $[\alpha]^{17} \mathrm{D}-84.68\left(\mathrm{c}=0.86, \mathrm{CH}_{2} \mathrm{Cl}_{2}\right) ;{ }^{1} \mathrm{H}$ NMR $\left(500 \mathrm{MHz}, \mathrm{CDCl}_{3}\right) \delta 7.29-7.39(\mathrm{~m}, 5 \mathrm{H})$, 5.70-5.79 (m, 1H), 5.36-5.37 (m, 1H), $4.41(\mathrm{dd}, J=1.97,7.90 \mathrm{~Hz}, 1 \mathrm{H}), 4.17-4.20(\mathrm{~m}, 1 \mathrm{H}), 3.89-3.92(\mathrm{~m}, 1 \mathrm{H})$, $3.43(\mathrm{qd}, J=6.18,8.09 \mathrm{~Hz}, 1 \mathrm{H}), 3.16(\mathrm{~d}, J=1.97 \mathrm{~Hz}, 1 \mathrm{H}), 1.32(\mathrm{~d}, J=6.18 \mathrm{~Hz}, 3 \mathrm{H}), 0.90(\mathrm{~s}, 9 \mathrm{H}), 0.10(\mathrm{~s}, 6 \mathrm{H})$; ${ }^{13} \mathrm{C} \mathrm{NMR}\left(125 \mathrm{MHz}, \mathrm{CDCl}_{3}\right) \delta 139.8,132.4,128.6,128.4,127.7,126.3,79.2,77.4,75.5,70.2,26.0,18.8,18.2$, 3.99, -4.44.; IR (NaCl) v 3466, 3089, 3065, 3035, 2956, 2858, 1495, 1471, 1455, 1390, $1361 \mathrm{~cm}^{-1}$; HRMS (ESI) $\mathrm{m} / \mathrm{z}:[\mathrm{M}+\mathrm{Na}]^{+}$Calcd for $\mathrm{C}_{19} \mathrm{H}_{30} \mathrm{O}_{3} \mathrm{SiNa} 357.1856$; Found 357.1854.<smiles>CC(C)O[C@H]1C=C[C@@H]([C@@H](O)c2ccc(F)cc2)O[C@H]1C</smiles>

(S)-((2S,5R,6S)-5-((tert-butyldimethylsilyl)oxy)-6-methyl-5,6-dihydro-2H-pyran-2-yl)(4-

fluorophenyl)methanol (4) : Following the general procedure using 4-fluorobenzaldehyde (24.8 $\mathrm{mg}, 0.20 \mathrm{mmol})$ 
and acetate $3(68.6 \mathrm{mg}, 0.24 \mathrm{mmol})$, Flash column chromatography (EtOAc:Hexane = 5:95) afforded the compound 4 as a colorless oil (58.6 mg, $0.166 \mathrm{mmol}, 83 \%$ yield). The diastereomer ratio ( $>25: 1)$ was determined by Crude NMR.

$\mathrm{R}_{f} 0.30$ (EtOAc:Hex = 10:90); $[\alpha]^{18} \mathrm{D}-78.82\left(\mathrm{c}=0.68, \mathrm{CH}_{2} \mathrm{Cl}_{2}\right) ;{ }^{1} \mathrm{H}$ NMR $\left(500 \mathrm{MHz}, \mathrm{CDCl}_{3}\right) \delta 7.33-7.36(\mathrm{~m}, 2 \mathrm{H})$, 7.02-7.06 (m, 2H), 5.71-5.73 (m, 1H), 5.32-5.35 (m, 1H), $4.40(\mathrm{dd}, J=1.44,7.88 \mathrm{~Hz}, 1 \mathrm{H}), 4.12-4.15(\mathrm{~m}, 1 \mathrm{H})$, $3.87-3.90(\mathrm{~m}, 1 \mathrm{H}), 3.42(\mathrm{qd}, J=6.13,8.03 \mathrm{~Hz}, 1 \mathrm{H}), 3.18(\mathrm{~d}, J=2.03 \mathrm{~Hz}, 1 \mathrm{H}), 1.31(\mathrm{~d}, J=6.13 \mathrm{~Hz}, 3 \mathrm{H}), 0.89(\mathrm{~s}$, 9H), 0.095 (s, 6H).; ${ }^{13} \mathrm{C}$ NMR $\left(125 \mathrm{MHz}, \mathrm{CDCl}_{3}\right) \delta 163.8,161.8,135.59,135.56,132.6,129.34,129.28,125.9$, 115.5, 115.4, 79.2, 76.7, 75.6, 70.2, 26.0, 18.8, 18.2, -4.01, -4.46.; IR (NaCl) v 3458, 3044, 2957, 2931, 2885, 2859, 1471, 1390, 1362, 1310, $1256 \mathrm{~cm}^{-1}$; HRMS (ESI) m/z: $[\mathrm{M}+\mathrm{Na}]^{+}$Calcd for $\mathrm{C}_{19} \mathrm{H}_{29} \mathrm{FO}_{3} \mathrm{SiNa} 375.1762$; Found 375.1761 .<smiles>C[C@H]1O[C@H]([C@@H](O)c2ccc(Cl)cc2)C=C[C@@H]1O[Mg]</smiles>

(S)-((2S,5R,6S)-5-((tert-butyldimethylsilyl)oxy)-6-methyl-5,6-dihydro-2H-pyran-2-yl)(4-

chlorophenyl)methanol (5): Following the general procedure using 4-chlorobenzaldehyde (28.0 mg, $0.20 \mathrm{mmol})$ and acetate $3(68.6 \mathrm{mg}, 0.24 \mathrm{mmol})$, Flash column chromatography (EtOAc:Hexane = 5:95) afforded the compound 5 as a colorless oil $(70.8 \mathrm{mg}, 0.192 \mathrm{mmol}, 96 \%$ yield $)$. The diastereomer ratio $(>25: 1)$ was determined by Crude NMR.

$\mathrm{R}_{f} 0.39$ (EtOAc:Hex = 10:90); $[\alpha]^{18}{ }_{\mathrm{D}}-91.25\left(\mathrm{c}=0.50, \mathrm{CH}_{2} \mathrm{Cl}_{2}\right) ;{ }^{1} \mathrm{H} \mathrm{NMR}\left(500 \mathrm{MHz}, \mathrm{CDCl}_{3}\right) \delta 7.30-7.34(\mathrm{~m}, 4 \mathrm{H})$, $5.73(\mathrm{~d}, J=10.43 \mathrm{~Hz}, 1 \mathrm{H}), 5.35(\mathrm{~d}, J=10.43 \mathrm{~Hz}, 1 \mathrm{H}), 4.40(\mathrm{~d}, J=7.61 \mathrm{~Hz}, 1 \mathrm{H}), 4.12-4.14(\mathrm{~m}, 1 \mathrm{H}), 3.87-3.89$ $(\mathrm{m}, 1 \mathrm{H}), 3.41(\mathrm{qd}, J=6.24,8.05 \mathrm{~Hz}, 1 \mathrm{H}), 3.17(\mathrm{~s}, 1 \mathrm{H}), 1.31(\mathrm{~d}, J=6.24 \mathrm{~Hz}, 3 \mathrm{H}), 0.90(\mathrm{~s}, 9 \mathrm{H}), 0.094(\mathrm{~s}, 6 \mathrm{H}) . ;{ }^{13} \mathrm{C}$ NMR $\left(125 \mathrm{MHz}, \mathrm{CDCl}_{3}\right) \delta 138.6,134.3,133.0,129.3,129.0,126.1,79.3,76.9,75.8,70.4,26.2,19.0,18.4,-3.75$, -4.20; IR (NaCl) v 3455, 3043, 2956, 2931, 2884, 2858, 1492, 1471, 1390, 1362, $1310 \mathrm{~cm}^{-1}$; HRMS (ESI) m/z: $[\mathrm{M}+\mathrm{Na}]^{+}$Calcd for $\mathrm{C}_{19} \mathrm{H}_{29} \mathrm{ClO}_{3} \mathrm{SiNa} 391.1467$; Found 391.1468.<smiles>CC(C)O[C@@H]1C=C[C@H](C)O[C@@H]1[C@H](O)c1ccc(Br)cc1</smiles>

(S)-(4-bromophenyl)((2S,5R,6S)-5-((tert-butyldimethylsilyl)oxy)-6-methyl-5,6-dihydro-2H-pyran-2yl)methanol (6): Following the general procedure using 4-bromobenzaldehyde (36.8 $\mathrm{mg}, 0.20 \mathrm{mmol}$ ) and acetate $3(68.6 \mathrm{mg}, 0.24 \mathrm{mmol})$, Flash column chromatography (EtOAc:Hexane = 5:95) afforded the compound 6 as a colorless oil $(58.6 \mathrm{mg}, 0.166 \mathrm{mmol}, 83 \%$ yield). The diastereomer ratio $(>25: 1)$ was determined by Crude NMR. $\mathrm{R}_{f} 0.31$ (EtOAc:Hex = 10:90); $[\alpha]^{17} \mathrm{D}-83.61\left(\mathrm{c}=0.51, \mathrm{CH}_{2} \mathrm{Cl}_{2}\right) ;{ }^{1} \mathrm{H}$ NMR $\left(500 \mathrm{MHz}, \mathrm{CDCl}_{3}\right) \delta 7.48-7.49(\mathrm{~m}, 2 \mathrm{H})$, 7.24-7.26 (m, 2H), $5.73(\mathrm{~d}, J=10.52 \mathrm{~Hz}, 1 \mathrm{H}), 5.36(\mathrm{~d}, J=10.52 \mathrm{~Hz}, 1 \mathrm{H}), 4.36(\mathrm{~d}, J=7.57 \mathrm{~Hz}, 1 \mathrm{H}), 4.12-4.13$ (m, 1H), 3.87-3.89 (m, 1H), 3.41 (qd, $J=6.15,8.10 \mathrm{~Hz}, 1 \mathrm{H}), 3.13-3.15(\mathrm{~m}, 1 \mathrm{H}), 1.30(\mathrm{~d}, J=6.15 \mathrm{~Hz}, 3 \mathrm{H}), 0.89$ (s, 9H), 0.093 (s, 6H).; ${ }^{13} \mathrm{C}$ NMR $\left(125 \mathrm{MHz}, \mathrm{CDCl}_{3}\right) \delta 138.9,132.8,131.7,129.3,125.9,122.3,79.0,76.7,75.6$, 70.1, 26.0, 18.8, 18.2, -3.99, -4.45.; IR (NaCl) v 3452, 3043, 2956, 2931, 2884, 2858, 1487, 1471, 1390, 1362 , $1310 \mathrm{~cm}^{-1}$; HRMS (ESI) m/z: [M + Na $]^{+}$Calcd for $\mathrm{C}_{19} \mathrm{H}_{29} \mathrm{BrO}_{3} \mathrm{SiNa} 435.0962$; Found 435.0963 .

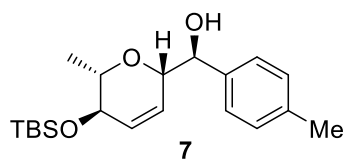

(S)-((2S,5R,6S)-5-((tert-butyldimethylsilyl)oxy)-6-methyl-5,6-dihydro-2H-pyran-2-yl)(p-tolyl)methanol (7) : Following the general procedure using 4-methylbenzaldehyde $(24.0 \mathrm{mg}, 0.20 \mathrm{mmol})$ and acetate $\mathbf{3}(68.6 \mathrm{mg}$, $0.24 \mathrm{mmol}$ ), Flash column chromatography (EtOAc:Hexane $=5: 95)$ afforded the compound 7 as a colorless oil (49.4 mg, $0.142 \mathrm{mmol}, 71 \%$ yield). The diastereomer ratio (11:1) was determined by Crude NMR

$\mathrm{R}_{f} 0.28$ (EtOAc:Hex = 10:90); $\left[\alpha{ }^{18}{ }_{\mathrm{D}}+81.58\left(\mathrm{c}=0.56, \mathrm{CH}_{2} \mathrm{Cl}_{2}\right) ;{ }^{1} \mathrm{H} \mathrm{NMR}\left(500 \mathrm{MHz}, \mathrm{CDCl}_{3}\right) \delta 7.26-7.28(\mathrm{~m}, 2 \mathrm{H})\right.$, 7.17-7.18 (m, 2H), 5.69-5.73 (m, 1H), 5.36-5.39 (m, 1H), $4.39(\mathrm{dd}, J=1.57,7.99 \mathrm{~Hz}, 1 \mathrm{H}), 4.16-4.19(\mathrm{~m}, 1 \mathrm{H})$, 3.90-3.93 (m, 1H), $3.44(\mathrm{qd}, J=6.17,8.09 \mathrm{~Hz}, 1 \mathrm{H}), 3.14(\mathrm{~d}, J=2.00 \mathrm{~Hz}, 1 \mathrm{H}), 2.36(\mathrm{~s}, 3 \mathrm{H}), 1.33(\mathrm{~d}, J=6.17 \mathrm{~Hz}$, 3H), 0.91 (s, 9H), 0.11 (s, 6H).; ${ }^{13} \mathrm{C}$ NMR (125 MHz, $\left.\mathrm{CDCl}_{3}\right) \delta 138.1,136.8,132.2,129.3,127.6,126.4,79.3$, 77.2, 75.5, 70.3, 26.0, 21.4, 18.8, 18.2, -4.00, -4.45.; IR (NaCl) v 2956, 2930, 2858, 1515, 1491, 1389, 1312, 1255 , $1196 \mathrm{~cm}^{-1}$; HRMS (ESI) m/z: [M + Na $]^{+}$Calcd for $\mathrm{C}_{20} \mathrm{H}_{32} \mathrm{O}_{3} \mathrm{SiNa} 371.2013$; Found 371.2014. 
<smiles></smiles>

(S)-((2S,5R,6S)-5-((tert-butyldimethylsilyl)oxy)-6-methyl-5,6-dihydro-2H-pyran-2-yl)(4-

methoxyphenyl)methanol (8) : Following the general procedure using 4-methoxybenzaldehyde (27.2 $\mathrm{mg}, 0.20$ $\mathrm{mmol})$ and acetate $3(68.6 \mathrm{mg}, 0.24 \mathrm{mmol})$, Flash column chromatography (EtOAc:Hexane $=5: 95)$ afforded the compound $\mathbf{8}$ as a colorless oil ( $31.6 \mathrm{mg}, 0.087 \mathrm{mmol}, 43 \%$ yield). The diastereomer ratio (6:1) was determined by Crude NMR

$\mathrm{R}_{f} 0.51$ (EtOAc:Hex $\left.=20: 80\right) ;[\alpha]^{18} \mathrm{D}-83.63\left(\mathrm{c}=1.51, \mathrm{CH}_{2} \mathrm{Cl}_{2}\right) ;{ }^{1} \mathrm{H}$ NMR $\left(500 \mathrm{MHz}, \mathrm{CDCl}_{3}\right) \delta$ 7.28-7.30 (m, 2H), 6.87-6.90 (m, 2H), 5.68-5.71 (m, 1H), 5.33-5.36 (m, 1H), $4.35(\mathrm{~d}, J=8.15 \mathrm{~Hz}, 1 \mathrm{H}), 4.14-4.17(\mathrm{~m}, 1 \mathrm{H}), 3.88-3.91$ (m, 1H), $3.81(\mathrm{~s}, 3 \mathrm{H}), 3.42(\mathrm{qd}, J=6.13,8.11 \mathrm{~Hz}, 1 \mathrm{H}), 3.12(\mathrm{brs}, 1 \mathrm{H}), 1.32(\mathrm{~d}, J=6.13 \mathrm{~Hz}, 3 \mathrm{H}), 0.89(\mathrm{~s}, 9 \mathrm{H})$, 0.093 (s, 6H).; ${ }^{13} \mathrm{C}$ NMR $\left(125 \mathrm{MHz}, \mathrm{CDCl}_{3}\right) \delta 159.5,132.0,131.7,128.7,127.3,126.2,113.8,79.1,75.3,70.1$, 55.3, 25.8, 18.6, 18.0, -4.20, -4.65.; IR ( NaCl) v 3470, 3042, 2958, 2931, 2884, 2858, 1614, 1586, 1514, 1464, $1390 \mathrm{~cm}^{-1}$; HRMS (ESI) m/z: [M + Na ${ }^{+}$Calcd for $\mathrm{C}_{20} \mathrm{H}_{32} \mathrm{O}_{4} \mathrm{SiNa}$ 387.1962; Found 387.1964.<smiles>Cc1ccccc1[C@H](O)[C@H]1C=C[C@@H](O[13CH3])[C@@H](C)O1</smiles>

(S)-((2S,5R,6S)-5-((tert-butyldimethylsilyl)oxy)-6-methyl-5,6-dihydro-2H-pyran-2-yl)(o-tolyl)methanol (9) : Following the general procedure using 2-methylbenzaldehyde $(24.0 \mathrm{mg}, 0.20 \mathrm{mmol})$ and acetate $3(68.6 \mathrm{mg}, 0.24$ $\mathrm{mmol}$ ), Flash column chromatography (EtOAc:Hexane $=5: 95)$ afforded the compound 9 as a colorless oil (53.6 $\mathrm{mg}, 0.154 \mathrm{mmol}, 77 \%$ yield). The diastereomer ratio (24:1) was determined by crude NMR

$\mathrm{R}_{f} 0.36$ (EtOAc:Hex = 10:90); $[\alpha]^{20}{ }_{\mathrm{D}}-66.35\left(\mathrm{c}=0.45, \mathrm{CH}_{2} \mathrm{Cl}_{2}\right) ;{ }^{1} \mathrm{H}$ NMR $\left(500 \mathrm{MHz}, \mathrm{CDCl}_{3}\right) \delta 7.47(\mathrm{~d}, J=7.31$ $\mathrm{Hz}, 1 \mathrm{H}), 7.14-7.25(\mathrm{~m}, 3 \mathrm{H}), 5.70(\mathrm{~d}, J=10.43 \mathrm{~Hz}, 1 \mathrm{H}), 5.29(\mathrm{~d}, J=10.43 \mathrm{~Hz}, 1 \mathrm{H}), 4.74(\mathrm{~d}, J=7.97 \mathrm{~Hz}, 1 \mathrm{H})$, 4.24-4.25 (m, 1H), 3.92-3.94 (m, 1H), $3.44(\mathrm{dq}, J=6.15,7.98 \mathrm{~Hz}, 1 \mathrm{H}), 3.10(\mathrm{~d}, J=1.46 \mathrm{~Hz}, 1 \mathrm{H}), 2.34(\mathrm{~s}, 1 \mathrm{H})$, $1.34(\mathrm{~d}, J=6.15 \mathrm{~Hz}, 3 \mathrm{H}), 0.90(\mathrm{~s}, 9 \mathrm{H}), 0.10(\mathrm{~s}, 6 \mathrm{H}) . ;{ }^{13} \mathrm{C} \mathrm{NMR}\left(125 \mathrm{MHz}, \mathrm{CDCl}_{3}\right) \delta 137.8,136.0,132.4,130.6$, 128.0, 127.3, 126.5, 126.2, 79.5, 75.5, 73.1, 70.3, 26.0, 20.0, 18.8, 18.2, -3.99, -4.44.; IR (NaCl) v 3468, 3041, 2956, 2931, 2858, 1492, 1464, 1380, 1308, $1265 \mathrm{~cm}^{-1}$; HRMS (ESI) m/z: $[\mathrm{M}+\mathrm{Na}]^{+}$Calcd for $\mathrm{C}_{20} \mathrm{H}_{32} \mathrm{O}_{3} \mathrm{SiNa}$ 371.2013; Found 371.2014.

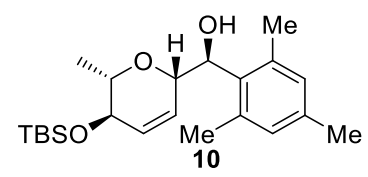

(S)-((2S,5R,6S)-5-((tert-butyldimethylsilyl)oxy)-6-methyl-5,6-dihydro-2H-pyran-2-yl)(mesityl)methanol

(10) : Following the general procedure using 2,4,6-trimethylbenzaldehyde $(29.6 \mathrm{mg}, 0.20 \mathrm{mmol})$ and acetate 3 $(68.6 \mathrm{mg}, 0.24 \mathrm{mmol}$ ), Flash column chromatography (EtOAc:Hexane $=5: 95)$ afforded the compound $\mathbf{1 0}$ as a colorless oil ( $31.3 \mathrm{mg}, 0.0832 \mathrm{mmol}, 42 \%$ yield). The diastereomer ratio $(>25: 1)$ was determined by isolation NMR

$\mathrm{R}_{f} 0.46$ (EtOAc:Hex $\left.=10: 90\right) ;[\alpha]^{21} \mathrm{D}-62.89\left(\mathrm{c}=1.52, \mathrm{CH}_{2} \mathrm{Cl}_{2}\right) ;{ }^{1} \mathrm{H}$ NMR $\left(500 \mathrm{MHz}, \mathrm{CDCl}_{3}\right) \delta 6.82(\mathrm{~s}, 2 \mathrm{H}), 5.65$ $(\mathrm{d}, J=10.54 \mathrm{~Hz}, 1 \mathrm{H}), 5.23(\mathrm{~d}, J=10.54 \mathrm{~Hz}, 1 \mathrm{H}), 4.90(\mathrm{~d}, J=9.23 \mathrm{~Hz}, 1 \mathrm{H}), 4.58-4.60(\mathrm{~m}, 1 \mathrm{H}), 3.93-3.95(\mathrm{~m}$, $1 \mathrm{H}), 3.45(\mathrm{qd}, J=6.25,7.84 \mathrm{~Hz}, 1 \mathrm{H}), 2.85(\mathrm{~s}, 1 \mathrm{H}), 2.39(\mathrm{~s}, 6 \mathrm{H}), 2.25(\mathrm{~s}, 3 \mathrm{H}), 1.35(\mathrm{~d}, J=6.25 \mathrm{~Hz}, 3 \mathrm{H}), 0.90(\mathrm{~s}$, 9H), $0.10(\mathrm{~s}, 3 \mathrm{H}), 0.099$ (s, 3H). ${ }^{13} \mathrm{C}$ NMR $\left(125 \mathrm{MHz}, \mathrm{CDCl}_{3}\right) \delta 137.5,137.4,131.9,131.8,130.4,126.8,76.8$, 75.5, 74.1, 70.3, 26.0, 21.5, 21.0, 18.9, 18.2, -3.97, -4.44.; IR (NaCl) v 3482, 3039, 2956, 2931, 2858, 1612, 1470 , 1378, 1361, 1312, $1255 \mathrm{~cm}^{-1}$; HRMS (ESI) m/z: $[\mathrm{M}+\mathrm{Na}]^{+}$Calcd for $\mathrm{C}_{22} \mathrm{H}_{36} \mathrm{O}_{3} \mathrm{SiNa}$ 399.2326; Found 399.2326.

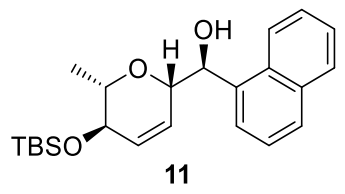


(S)-((2S,5R,6S)-5-((tert-butyldimethylsilyl)oxy)-6-methyl-5,6-dihydro-2H-pyran-2-yl)(naphthalen-1yl)methanol (11): Following the general procedure using 1-naphthaldehyde (31.2 $\mathrm{mg}, 0.20 \mathrm{mmol})$ and acetate 3 (68.6 mg, $0.24 \mathrm{mmol})$, Flash column chromatography (EtOAc:Hexane = 5:95) afforded the compound 11 as a colorless oil $(65.0 \mathrm{mg}, 0.169 \mathrm{mmol}, 85 \%$ yield). The diastereomer ratio $(>25: 1)$ was determined by isolation NMR $\mathrm{R}_{f} 0.38$ (EtOAc:Hex = 10:90); $[\alpha]^{17}{ }_{\mathrm{D}}-53.18\left(\mathrm{c}=0.50, \mathrm{CH}_{2} \mathrm{Cl}_{2}\right) ;{ }^{1} \mathrm{H}$ NMR $\left(500 \mathrm{MHz}, \mathrm{CDCl}_{3}\right) \delta 8.11(\mathrm{~d}, J=8.15$ $\mathrm{Hz}, 1 \mathrm{H}), 7.87-7.89(\mathrm{~m}, 1 \mathrm{H}), 7.82(\mathrm{~d}, J=8.15 \mathrm{~Hz}, 1 \mathrm{H}), 7.70(\mathrm{~d}, J=7.15 \mathrm{~Hz}, 1 \mathrm{H}), 7.49-7.53(\mathrm{~m}, 3 \mathrm{H}), 5.67(\mathrm{~d}, J=$ $10.38 \mathrm{~Hz}, 1 \mathrm{H}), 5.24-5.29(\mathrm{~m}, 2 \mathrm{H}), 4.47-4.49(\mathrm{~m}, 1 \mathrm{H}), 3.97-3.99(\mathrm{~m}, 1 \mathrm{H}), 3.47$ (qd $J=6.31,7.98 \mathrm{~Hz}, 1 \mathrm{H}), 3.32-$ $3.33(\mathrm{~m}, 1 \mathrm{H}), 1.36(\mathrm{~d}, J=6.31 \mathrm{~Hz}, 3 \mathrm{H}), 0.90(\mathrm{~s}, 9 \mathrm{H}), 0.11(\mathrm{~s}, 3 \mathrm{H}), 0.10(\mathrm{~s}, 3 \mathrm{H}) . ;{ }^{13} \mathrm{C} \mathrm{NMR}\left(125 \mathrm{MHz}, \mathrm{CDCl}_{3}\right) \delta$ $135.5,134.0,132.2,131.5,129.1,128.8,126.7,126.2,125.69,125.67,125.59,123.8,79.3,75.6,73.6,70.3,26.0$, 18.9, 18.2, -3.97, -4.43.; IR (NaCl) v 3467, 3049, 2956, 2930, 2884, 2858, 1512, 1470, 1390, 1360, $1312 \mathrm{~cm}^{-1}$; HRMS (ESI) m/z: $[\mathrm{M}+\mathrm{Na}]^{+}$Calcd for $\mathrm{C}_{23} \mathrm{H}_{32} \mathrm{O}_{3} \mathrm{SiNa}$ 407.2013; Found 407.2015.<smiles>C[C@H]1O[C@H]([C@@H](O)C2CCCCC2)C=C[C@@H]1OC(C)(C)C</smiles>

(S)-((2S,5R,6S)-5-((tert-butyldimethylsilyl)oxy)-6-methyl-5,6-dihydro-2H-pyran-2-

yl)(cyclohexyl)methanol (12) : Following the general procedure using cyclohexanecarbaldehyde $(67.2 \mathrm{mg}, 0.60$ $\mathrm{mmol}$ ) and acetate $3(57.2 \mathrm{mg}, 0.20 \mathrm{mmol})$, Flash column chromatography (EtOAc:Hexane = 4:96) afforded the compound 12 as a colorless oil (44.2 $\mathrm{mg}, 0.130 \mathrm{mmol}, 65 \%$ yield). The diastereomer ratio (7:1) was determined by crude NMR

$\mathrm{R}_{f} 0.50$ (EtOAc:Hex = 10:90); $[\alpha]^{15} \mathrm{D}-87.68\left(\mathrm{c}=1.66, \mathrm{CH}_{2} \mathrm{Cl}_{2}\right) ;{ }^{1} \mathrm{H}$ NMR $\left(500 \mathrm{MHz}, \mathrm{CDCl}_{3}\right) \delta 5.77-5.79(\mathrm{~m}, 1 \mathrm{H})$, 5.66-5.68 (m, 1H), 4.18-4.20 (m, 1H), 3.86-3.89 (m, 1H), 3.39 (qd, $J=6.10,8.04 \mathrm{~Hz}, 1 \mathrm{H}), 3.14-3.17(\mathrm{~m}, 1 \mathrm{H})$, $2.08(\mathrm{~d}, J=6.12 \mathrm{~Hz}, 1 \mathrm{H}), 1.86-1.89(\mathrm{~m}, 1 \mathrm{H}), 1.73-1.78(\mathrm{~m}, 2 \mathrm{H}), 1.64-1.67(\mathrm{~m}, 2 \mathrm{H}), 1.51-1.58(\mathrm{~m}, 1 \mathrm{H}), 1.06-1.31$ $(\mathrm{m}, 8 \mathrm{H}), 0.89$ (s, 9H), 0.095 (s, 3H), 0.084 (s, 3H).; ${ }^{13} \mathrm{C}$ NMR $\left(125 \mathrm{MHz}, \mathrm{CDCl}_{3}\right) \delta 132.3,128.4,77.8,75.5,75.0$, 70.3, 40.0, 29.9, 28.3, 26.63., 26.55, 26.3, 26.0, 18.9, 18.2, -3.97, -4.46.; IR (NaCl) v 3466, 3039, 2929, 2856, 1450, 1389, 1361, 1310, 1255, 1186, $1138 \mathrm{~cm}^{-1}$; HRMS (ESI) m/z: [M + Na $]^{+}$Calcd for $\mathrm{C}_{19} \mathrm{H}_{36} \mathrm{O}_{3} \mathrm{SiNa} 363.2326$; Found 363.2327<smiles>C[C@H](O)[C@H](O)[C@H]1C=C[C@@H](OS(C)(=O)=O)[C@@H](C)O1</smiles>

(S)-1-((2S,5R,6S)-5-((tert-butyldimethylsilyl)oxy)-6-methyl-5,6-dihydro-2H-pyran-2-yl)tridecan-1-ol (11) : Following the general procedure using tridecanal $(119 \mathrm{mg}, 0.60 \mathrm{mmol})$ and acetate $3(57.2 \mathrm{mg}, 0.20 \mathrm{mmol})$, Flash column chromatography (EtOAc:Hexane = 4:96) afforded the compound 13 as a colorless oil $(60.5 \mathrm{mg}, 0.142$ mmol, $71 \%$ yield). The diastereomer ratio $(8: 1)$ was determined by isolation NMR

$\mathrm{R}_{f} 0.37$ (EtOAc:Hex = 10:90); $[\alpha]^{16}{ }_{\mathrm{D}}-82.99\left(\mathrm{c}=1.02, \mathrm{CH}_{2} \mathrm{Cl}_{2}\right) ;{ }^{1} \mathrm{H}$ NMR $\left(500 \mathrm{MHz}, \mathrm{CDCl}_{3}\right) \delta 5.77(\mathrm{~d}, J=10.36$ $\mathrm{Hz}, 1 \mathrm{H}), 5.68(\mathrm{~d}, J=10.36 \mathrm{~Hz}, 1 \mathrm{H}), 3.98-3.97(\mathrm{~m}, 1 \mathrm{H}), 3.86-3.87(\mathrm{~m}, 1 \mathrm{H}), 3.37-3.47(\mathrm{~m}, 2 \mathrm{H}), 2.27(\mathrm{~d}, J=4.54$ $\mathrm{Hz}, 1 \mathrm{H}), 1.46-1.54(\mathrm{~m}, 3 \mathrm{H}), 1.23-1.37(\mathrm{~m}, 24 \mathrm{H}), 0.86-0.92(\mathrm{~m}, 12 \mathrm{H}), 0.10(\mathrm{~s}, 3 \mathrm{H}), 0.09(\mathrm{~s}, 3 \mathrm{H}) . ;{ }^{13} \mathrm{C} \mathrm{NMR}(125$ $\left.\mathrm{MHz}, \mathrm{CDCl}_{3}\right) \delta 132.3,127.5,77.7,75.5,73.8,70.4,32.9,32.1,29.89,29.87,29.83,29.81,29.6,26.0,25.8,22.9$, 18.8, 18.2, 14.3, -4.00, -4.50.; IR (NaCl) v 3464, 3041, 2927, 2856, 1465, 1380, 1362, 1312, $1094 \mathrm{~cm}^{-1}$; HRMS (ESI) $\mathrm{m} / \mathrm{z}:[\mathrm{M}+\mathrm{Na}]^{+}$Calcd for $\mathrm{C}_{25} \mathrm{H}_{50} \mathrm{O}_{3} \mathrm{SiNa} 449.3421$; Found 449.3423.<smiles>C[C@@H]1O[C@H]([C@H](O)CO[SbH3])C=C[C@H]1O[SbH3]</smiles>

(S)-2-((tert-butyldimethylsilyl)oxy)-1-((2S,5R,6S)-5-((tert-butyldimethylsilyl)oxy)-6-methyl-5,6-dihydro2H-pyran-2-yl)ethan-1-ol (14) : Following the general procedure using 2-((tertbutyldimethylsilyl)oxy)acetaldehyde $(104 \mathrm{mg}, 0.60 \mathrm{mmol})$ and acetate $3(57.2 \mathrm{mg}, 0.20 \mathrm{mmol})$, Flash column chromatography (EtOAc:Hexane = 4:96) afforded the compound 14 as a colorless oil $(65.2 \mathrm{mg}, 0.162 \mathrm{mmol}, 81 \%$ 
yield). The diastereomer ratio (8:1) was determined by isolation NMR.

$\mathrm{R}_{f} 0.46$ (EtOAc:Hex = 10:90); $[\alpha]^{15} \mathrm{D}-69.40\left(\mathrm{c}=2.10, \mathrm{CH}_{2} \mathrm{Cl}_{2}\right) ;{ }^{1} \mathrm{H}$ NMR $\left(500 \mathrm{MHz}, \mathrm{CDCl}_{3}\right) \delta 5.77-5.79(\mathrm{~m}, 1 \mathrm{H})$, 5.73-5.76 (m, 1H), 4.26-4.28 (m, 1H), 3.87-3.89 (m, 1H), 3.63-3.71 (m, 2H), 3.58-3.62 (m, 1H), $3.41(\mathrm{qd}, J=$ $6.20,8.00 \mathrm{~Hz}, 1 \mathrm{H}), 2.40(\mathrm{~d}, J=5.48 \mathrm{~Hz}, 1 \mathrm{H}), 1.26(\mathrm{~d}, J=6.20 \mathrm{~Hz}, 3 \mathrm{H}), 0.90(\mathrm{~s}, 9 \mathrm{H}), 0.89$ (s, 9H), $0.098(\mathrm{~s}, 3 \mathrm{H})$, $0.086(\mathrm{~s}, 3 \mathrm{H}), 0.070(\mathrm{~s}, 3 \mathrm{H}), 0.067(\mathrm{~s}, 3 \mathrm{H}) . ;{ }^{13} \mathrm{C} \mathrm{NMR}\left(125 \mathrm{MHz}, \mathrm{CDCl}_{3}\right) \delta 132.1,127.9,75.6,74.5,73.5,70.3$, 63.4, 26.09, 25.99, 18.8, 18.5, 18.2, -3.97, -4.47, -5.18, -5.21.; IR (NaCl) v 3474, 3041, 2956, 2931, 2885, 2858, 1471, 1389, 1362, 1310, 1255, $1150 \mathrm{~cm}^{-1}$; HRMS (ESI) m/z: [M+Na $]^{+}$Calcd for $\mathrm{C}_{20} \mathrm{H}_{42} \mathrm{O}_{4} \mathrm{Si}_{2} \mathrm{Na}_{425.2514}$; Found 425.2515 .

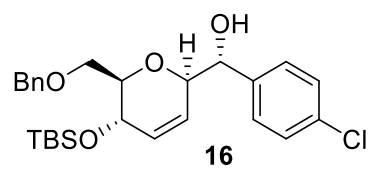

(R)-((2R,5S,6R)-6-((benzyloxy)methyl)-5-((tert-butyldimethylsilyl)oxy)-5,6-dihydro-2H-pyran-2-yl)(4chlorophenyl)methanol (16) : Following the general procedure using 4-chlorobenzaldehyde $(28.0 \mathrm{mg}, 0.20$ mmol) and acetate $15(94.1 \mathrm{mg}, 0.24 \mathrm{mmol})$, Flash column chromatography (EtOAc:Hexane = 20:80) afforded the compound 16 as a colorless oil $(85.3 \mathrm{mg}, 0.180 \mathrm{mmol}, 90 \%$ yield). The diastereomer ratio ( $>25: 1)$ was determined by Crude NMR.

$\mathrm{R}_{f} 0.31$ (EtOAc: Hex = 20:80); $[\alpha]^{16}{ }_{\mathrm{D}}+67.28\left(\mathrm{c}=0.52 \mathrm{CH}_{2} \mathrm{Cl}_{2}\right) ;{ }^{1} \mathrm{H}$ NMR $\left(500 \mathrm{MHz}, \mathrm{CDCl}_{3}\right) \delta 7.29-7.37(\mathrm{~m}, 9 \mathrm{H})$, $5.75(\mathrm{~d}, J=10.27 \mathrm{~Hz}, 1 \mathrm{H}), 5.41(\mathrm{~d}, J=10.27 \mathrm{~Hz}, 1 \mathrm{H}), 4.62(\mathrm{~d}, J=12.22 \mathrm{~Hz}, 1 \mathrm{H}), 4.54(\mathrm{~d}, J=12.22 \mathrm{~Hz}, 1 \mathrm{H})$, $4.47(\mathrm{dd}, J=2.13,7.14 \mathrm{~Hz}, 1 \mathrm{H}), 4.19-4.21(\mathrm{~m}, 2 \mathrm{H}), 3.75(\mathrm{dd}, J=1.74,10.65 \mathrm{~Hz}, 1 \mathrm{H}), 3.64(\mathrm{dd}, J=5.50,10.65$ $\mathrm{Hz}, 1 \mathrm{H}), 3.50-3.52(\mathrm{~m}, 1 \mathrm{H}), 3.29(\mathrm{~d}, J=2.13 \mathrm{~Hz}, 1 \mathrm{H}), 0.86(\mathrm{~s}, 9 \mathrm{H}), 0.076(\mathrm{~s}, 3 \mathrm{H}), 0.038(\mathrm{~s}, 3 \mathrm{H})$; ${ }^{13} \mathrm{C}$ NMR $(125$ $\left.\mathrm{MHz}, \mathrm{CDCl}_{3}\right) \delta 138.40,138.37,134.0,132.5,129.0,128.7,128.6,127.9,126.0,79.1,79.0,76.3,73.6,69.7,64.2$, 25.1, 18.1, -4.04, -4.69.; IR (NaCl) v 3422, 3033, 2954, 2929, 2884, 2857, 1492, 1470, 1387, 1361, 1313, 1254 $\mathrm{cm}^{-1}$; HRMS (ESI) m/z: [M + Na $]^{+}$Calcd for $\mathrm{C}_{26} \mathrm{H}_{35} \mathrm{ClO}_{4} \mathrm{SiNa} 497.1885$; Found 497.1889.<smiles>CO[C@H]1C=C[C@@H]([C@@H](O)c2ccc(Cl)cc2)[C@H](C)O1</smiles>

(S)-(4-chlorophenyl)((2S,5R,6S)-6-methyl-5-((trimethylsilyl)oxy)-5,6-dihydro-2H-pyran-2-yl)methanol (19) : Following the general procedure using 4-chlorobenzaldehyde (28.0 mg, $0.20 \mathrm{mmol})$ and acetate 17 (58.6 $\mathrm{mg}, 0.24 \mathrm{mmol}$ ), Flash column chromatography (EtOAc:Hexane =10:90) afforded the compound 19 as a colorless oil (44.1 mg, $0.135 \mathrm{mmol}, 68 \%$ yield). The diastereomer ratio $(>25: 1)$ was determined by Crude NMR.

$\mathrm{R}_{f} 0.18$ (EtOAc:Hex = 10:90); $[\alpha]^{22}{ }_{\mathrm{D}}-92.04\left(\mathrm{c}=0.84, \mathrm{CH}_{2} \mathrm{Cl}_{2}\right) ;{ }^{1} \mathrm{H}$ NMR $\left(500 \mathrm{MHz}, \mathrm{CDCl}_{3}\right) \delta 7.28-7.34(\mathrm{~m}, 4 \mathrm{H})$, $5.70(\mathrm{~d}, J=10.56 \mathrm{~Hz}, 1 \mathrm{H}), 5.39$ (d, $J=10.56 \mathrm{~Hz}, 1 \mathrm{H}), 4.40(\mathrm{~d}, J=7.57 \mathrm{~Hz}, 1 \mathrm{H}), 4.12-4.13(\mathrm{~m}, 1 \mathrm{H}), 3.87-3.89$ $(\mathrm{m}, 1 \mathrm{H}), 3.42(\mathrm{qd}, J=6.18,7.99 \mathrm{~Hz}, 1 \mathrm{H}), 3.14$ (brs, $1 \mathrm{H}), 1.30(\mathrm{~d}, J=6.18 \mathrm{~Hz}, 3 \mathrm{H}), 0.15(\mathrm{~s}, 9 \mathrm{H})$; ${ }^{13} \mathrm{C}$ NMR $(125$ $\left.\mathrm{MHz} \mathrm{CDCl}_{3}\right) \delta 138.3,134.1,132.6,129.0,128.8,126.1,79.0,76.7,75.4,69.9,18.7,0.44$.; IR (NaCl) v 3448, 3042, 2958, 1492, 1450, 1393, 1379, 1252, 1196, 1131, $1096 \mathrm{~cm}^{-1}$; HRMS (ESI) m/z: $[\mathrm{M}+\mathrm{Na}]^{+}$Calcd for $\mathrm{C}_{16} \mathrm{H}_{23} \mathrm{ClO}_{3} \mathrm{SiNa} 349.0997$; Found 349.0999.<smiles>C[C@H]1O[C@H]([C@@H](O)c2ccc(Cl)cc2)C=C[C@H]1OC(C)(C)C</smiles>

(S)-((2S,5R,6S)-5-((tert-butyldiphenylsilyl)oxy)-6-methyl-5,6-dihydro-2H-pyran-2-yl)(4-

chlorophenyl)methanol (20) : Following the general procedure using 4-chlorobenzaldehyde (28.0 $\mathrm{mg}, 0.20$ $\mathrm{mmol})$ and acetate $18(98.4 \mathrm{mg}, 0.24 \mathrm{mmol})$, Flash column chromatography (EtOAc:Hexane = 10:90) afforded the compound 20 as a colorless oil $(78.6 \mathrm{mg}, 0.160 \mathrm{mmol}, 80 \%$ yield). The diastereomer ratio $(>25: 1)$ was determined by Crude NMR.

$\mathrm{R}_{f} 0.19$ (EtOAc:Hex = 10:90); $[\alpha]^{22}{ }_{\mathrm{D}}-98.17\left(\mathrm{c}=1.335, \mathrm{CH}_{2} \mathrm{Cl}_{2}\right) ;{ }^{1} \mathrm{H}$ NMR $\left(500 \mathrm{MHz}, \mathrm{CDCl}_{3}\right) \delta$ 7.65-7.69 (m, 4H), 7.35-7.49 (m, 6H), 7.25-7.31 (m, 4H), $5.64(\mathrm{~d}, J=10.49 \mathrm{~Hz}, 1 \mathrm{H}), 5.24(\mathrm{~d}, J=10.49 \mathrm{~Hz}, 1 \mathrm{H}), 4.29-4.31$ (m, 
$1 \mathrm{H}), 4.09-4.11(\mathrm{~m}, 1 \mathrm{H}), 3.98-3.99(\mathrm{~m}, 1 \mathrm{H}), 3.57$ (qd, $J=6.20,7.83 \mathrm{~Hz}, 1 \mathrm{H}), 3.09$ (d, $J=1.80 \mathrm{~Hz}, 1 \mathrm{H}), 1.26(\mathrm{~d}, J$ $=6.20 \mathrm{~Hz}, 3 \mathrm{H}), 1.06(\mathrm{~s}, 9 \mathrm{H}) \cdot ;{ }^{13} \mathrm{C} \mathrm{NMR}\left(125 \mathrm{MHz}, \mathrm{CDCl}_{3}\right) \delta 138.2,136.13,136.11,134.3,134.1,133.6,132.2$, $130.1,129.9,129.0,128.7,127.9,127.8,125.8,78.9,76.5,75.7,71.1,27.2,19.6,19.0 . ; \mathrm{IR}(\mathrm{NaCl}) \vee 3449,3078$, 3048, 2961, 2932, 2858, 1489, 1472, 1428, 1391, 1380, 1256, 1194, $1015 \mathrm{~cm}^{-1}$; HRMS (ESI) m/z: [M + Na] ${ }^{+}$ Calcd for $\mathrm{C}_{29} \mathrm{H}_{33} \mathrm{ClO}_{3} \mathrm{SiNa} 515.1780$; Found 515.1782.

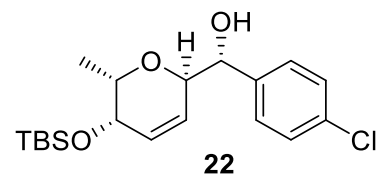

(S)-((2S,5S,6S)-5-((tert-butyldimethylsilyl)oxy)-6-methyl-5,6-dihydro-2H-pyran-2-yl)(4-

chlorophenyl)methanol (22) : Following the general procedure using 4-chlorobenzaldehyde (28.0 mg, 0.20 $\mathrm{mmol}$ ) and acetate 21 (68.6 mg, $0.24 \mathrm{mmol})$, Flash column chromatography (EtOAc:Hexane = 5:95) afforded the compound 22 as a colorless oil (46.3 mg, $0.126 \mathrm{mmol}, 63 \%$ yield) and C3-product $\mathbf{S 8}$ (18.2 $\mathrm{mg}, 0.0494 \mathrm{mmol}$, $25 \%$ yield). The diastereomer ratio (19:1) was determined by Crude NMR.

$\mathrm{R}_{f} 0.24$ (EtOAc:Hex = 10:90); $\left[\alpha{ }^{18}{ }_{\mathrm{D}}+144.90\left(\mathrm{c}=2.04, \mathrm{CH}_{2} \mathrm{Cl}_{2}\right) ;{ }^{1} \mathrm{H}\right.$ NMR $\left(500 \mathrm{MHz}, \mathrm{CDCl}_{3}\right) \delta$ 7.28-7.34 (m, $4 \mathrm{H}), 5.81-5.84(\mathrm{~m}, 1 \mathrm{H}), 5.36-5.39(\mathrm{~m}, 1 \mathrm{H}), 4.56(\mathrm{~d}, J=8.30 \mathrm{~Hz}, 1 \mathrm{H}), 4.03-4.09(\mathrm{~m}, 3 \mathrm{H}), 3.21(\mathrm{brs}, 1 \mathrm{H}), 1.24(\mathrm{~d}$, $J=6.35 \mathrm{~Hz}, 3 \mathrm{H}), 0.90(\mathrm{~s}, 9 \mathrm{H}), 0.081(\mathrm{~s}, 3 \mathrm{H}), 0.075(\mathrm{~s}, 3 \mathrm{H}) . ;{ }^{13} \mathrm{C} \mathrm{NMR}\left(125 \mathrm{MHz}, \mathrm{CDCl}_{3}\right) \delta 138.4,134.1,129.8$, 128.8, 126.2, 76.0, 74.4, 70.2, 65.1, 26.0, 18.4, 15.2, -4.18, -4.46.; IR (NaCl) v 3449, 3042, 2955, 2931, 2886, 2858, 1492, 1471, 1390, 1361, $1255 \mathrm{~cm}^{-1}$; HRMS (ESI) m/z: $[\mathrm{M}+\mathrm{Na}]^{+}$Calcd for $\mathrm{C}_{19} \mathrm{H}_{29} \mathrm{ClO}_{3} \mathrm{SiNa} 391.1467$; Found 391.1469.

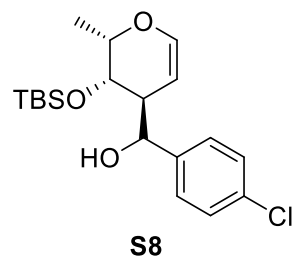

((2S,3S)-3-((tert-butyldimethylsilyl)oxy)-2-methyl-3,4-dihydro-2H-pyran-4-yl)(4-chlorophenyl)methanol (S8)

$\mathrm{R}_{f} 0.31$ (EtOAc:Hex = 10:90); m.p 90-91 ${ }^{\circ} \mathrm{C}$; $[\alpha]^{18}{ }_{\mathrm{D}}-68.61\left(\mathrm{c}=0.85, \mathrm{CH}_{2} \mathrm{Cl}_{2}\right) ;{ }^{1} \mathrm{H}$ NMR $\left(500 \mathrm{MHz}, \mathrm{CDCl}_{3}\right) \delta 7.39$ $(\mathrm{d}, J=8.42 \mathrm{~Hz}, 2 \mathrm{H}), 7.28(\mathrm{~d}, J=8.42 \mathrm{~Hz}, 2 \mathrm{H}), 6.30(\mathrm{dd}, J=1.60,6.21 \mathrm{~Hz}, 1 \mathrm{H}), 4.45(\mathrm{~d}, J=8.25 \mathrm{~Hz}, 1 \mathrm{H}), 4.01-$ 4.03 (m, 3H), 2.69 (brs, 1H), 2.33-2.35 (m, 1H), 1.26 (d, J=6.35 Hz, 3H), 0.91 (s, 9H), $0.093(\mathrm{~s}, 3 \mathrm{H}), 0.086(\mathrm{~s}$, $3 \mathrm{H})$; ${ }^{13} \mathrm{C}$ NMR $\left(125 \mathrm{MHz}, \mathrm{CDCl}_{3}\right) \delta 144.3,140.7,133.7,128.7,128.5,97.4,77.3,71.6,70.1,45.7,26.0,18.2$, 15.7, -4.09, -4.50; IR (NaCl) v 3497, 3065, 2954, 2930, 2886, 2857, 1651, 1490, 1471, 1409, $1378 \mathrm{~cm}^{-1}$; HRMS (ESI) $\mathrm{m} / \mathrm{z}:[\mathrm{M}+\mathrm{Na}]^{+}$Calcd for $\mathrm{C}_{19} \mathrm{H}_{29} \mathrm{ClO}_{3} \mathrm{SiNa} 391.1467$; Found 391.1468.<smiles>[Z4]c1ccc([C@@H](O)[C@@H]2C=CC[C@@H](CO[B])O2)cc1</smiles>

(R)-((2R,6S)-6-((benzyloxy)methyl)-5,6-dihydro-2H-pyran-2-yl)(4-chlorophenyl)methanol (24) : Following the general procedure using 4-chlorobenzaldehyde $(28.0 \mathrm{mg}, 0.20 \mathrm{mmol})$ and acetate $23(62.9 \mathrm{mg}, 0.24 \mathrm{mmol})$, Flash column chromatography (EtOAc:Hexane $=20: 80)$ afforded the compound 24 as a colorless oil (49.6 mg, $0.144 \mathrm{mmol}, 72 \%$ yield $), \alpha$-product $(0.0126 \mathrm{mmol}, 6 \%$ yield $)$ and $C 3$-product $(0.028 \mathrm{mmol}, 14 \%$ yield $)$. The diastereomer ratio $(9: 1)$ was determined by Crude NMR.

$\mathrm{R}_{f} 0.29$ (EtOAc:Hex = 20:80); $[\alpha]^{12}{ }_{\mathrm{D}}-41.57\left(\mathrm{c}=0.88, \mathrm{CH}_{2} \mathrm{Cl}_{2}\right) ;{ }^{1} \mathrm{H}$ NMR $\left(500 \mathrm{MHz}, \mathrm{CDCl}_{3}\right) \delta 7.27-7.38(\mathrm{~m}, 9 \mathrm{H})$, 5.86-5.90 (m, 1H), 5.37-5.40 (m, 1H), $4.58(\mathrm{~s}, 2 \mathrm{H}), 4.47$ (d, J=7.45 Hz, 1H), 4.20-4.22 (m, 1H), 3.85-3.90 (m, $1 \mathrm{H}), 3.59$ (dd, $J=6.28,10.38 \mathrm{~Hz}, 1 \mathrm{H}), 3.54(\mathrm{dd}, J=4.06,10.38 \mathrm{~Hz}, 1 \mathrm{H}), 3.24(\mathrm{~s}, 1 \mathrm{H}), 2.06-2.14(\mathrm{~m}, 1 \mathrm{H}), 1.95-$ $2.01(\mathrm{~m}, 1 \mathrm{H}) . ;{ }^{13} \mathrm{C} \mathrm{NMR}\left(125 \mathrm{MHz}, \mathrm{CDCl}_{3}\right) \delta 138.6,138.4,134.0,129.1,128.65,128.64,127.92,127.87,126.9$, 125.7, 79.0, 76.3, 73.6, 73.4, 73.0, 27.7.; IR (NaCl) v 3435, 3033, 2890, 2862, 1491, 1453, 1365, 1310, 1258 , 
1188, 1088, $1014 \mathrm{~cm}^{-1}$; HRMS (ESI) m/z: $[\mathrm{M}+\mathrm{Na}]^{+}$Calcd for $\mathrm{C}_{20} \mathrm{H}_{21} \mathrm{ClO}_{3} \mathrm{Na}$ 367.1071; Found 367.1070.
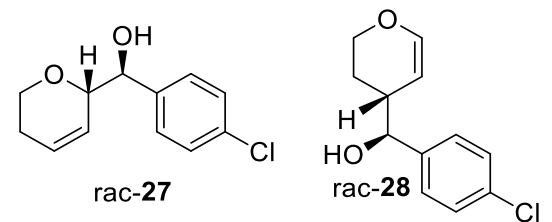

(4-chlorophenyl)(5,6-dihydro-2H-pyran-2-yl)methanol (rac-27) : Following the general procedure using 4chlorobenzaldehyde (28.0 mg, $0.20 \mathrm{mmol}$ ) and mixture of acetate $\mathbf{2 5}$ and $\mathbf{2 6}^{5}$ (34.1 $\left.\mathrm{mg}, 0.24 \mathrm{mmol}, 5: 1\right)$, Flash column chromatography (EtOAc:Toluene $=5: 95)$ afforded the compound rac-27 as a colorless oil $(25.5 \mathrm{mg}, 0.114$ mmol, $57 \%$ yield) and $C 3$ product rac-28 $(7.2 \mathrm{mg}, 0.032 \mathrm{mmol}, 16 \%$ yield). The diastereomer ratio (7:1) was determined by Isolation NMR.

$\mathrm{R}_{f} 0.50$ (EtOAc:Toluene $\left.=20: 80\right) ;{ }^{1} \mathrm{H}$ NMR $\left(500 \mathrm{MHz}, \mathrm{CDCl}_{3}\right) \delta$ 7.30-7.34 (m, 4H), 5.91-5.95 (m, 1H), 5.33-5.36 $(\mathrm{m}, 1 \mathrm{H}), 4.53$ (dd, $J=1.96,7.78 \mathrm{~Hz}, 1 \mathrm{H}), 4.06-4.10(\mathrm{~m}, 1 \mathrm{H}), 4.00-4.04$ (m, 1H), 3.72 (ddd, $J=4.24,8.38,11.32$ $\mathrm{Hz}, 1 \mathrm{H}), 3.19$ (brs, $1 \mathrm{H}), 2.20-2.28(\mathrm{~m}, 1 \mathrm{H}), 2.00-2.07(\mathrm{~m}, 1 \mathrm{H}) . ;{ }^{13} \mathrm{C}$ NMR $\left(125 \mathrm{MHz}, \mathrm{CDCl}_{3}\right) \delta 138.6,133.9$, 128.8, 128.7, 127.4, 125.6, 78.1, 75.9, 62.9, 25.3.; IR (NaCl) v 3447, 3041, 2967, 2918, 2900, 2864, 2836, 1490, 1429, 1398, 1374, $1267 \mathrm{~cm}^{-1}$; HRMS (ESI) m/z: [M + Na] $]^{+}$Calcd for $\mathrm{C}_{12} \mathrm{H}_{13} \mathrm{ClO}_{2} \mathrm{Na} 247.0496$; Found 247.0499. (4-chlorophenyl)(3,4-dihydro-2H-pyran-4-yl)methanol (rac-28) :

$\mathrm{R}_{f} 0.55$ (EtOAc:Toluene = 20:80); ${ }^{1} \mathrm{H}$ NMR $\left(500 \mathrm{MHz}, \mathrm{CDCl}_{3}\right) \delta$ 7.27-7.33 (m, 4H), 6.43-6.45 (m, 1H), 4.48-4.50 $(\mathrm{m}, 1 \mathrm{H}), 4.30-4.32(\mathrm{~m}, 1 \mathrm{H}), 4.03-4.07(\mathrm{~m}, 1 \mathrm{H}), 3.90-3.94(\mathrm{~m}, 1 \mathrm{H}), 2.47-2.51(\mathrm{~m}, 1 \mathrm{H}), 1.79-1.99(\mathrm{~m}, 3 \mathrm{H}) . ;{ }^{13} \mathrm{C}$ NMR $\left(125 \mathrm{MHz}, \mathrm{CDCl}_{3}\right) \delta 146.6,141.0,133.6,128.7,128.1,101.0,76.7,64.6,38.1,24.6$; IR (NaCl) v 3423, 3062, 2970, 2933, 2877, 1649, 1490, 1463, 1438, 1408, 1338, $1243 \mathrm{~cm}^{-1}$; HRMS (ESI) m/z: [M + Na] Calcd for $\mathrm{C}_{12} \mathrm{H}_{13} \mathrm{ClO}_{2} \mathrm{SiNa} 247.0496$; Found 247.0494.

\section{Formal synthesis of mucocin}
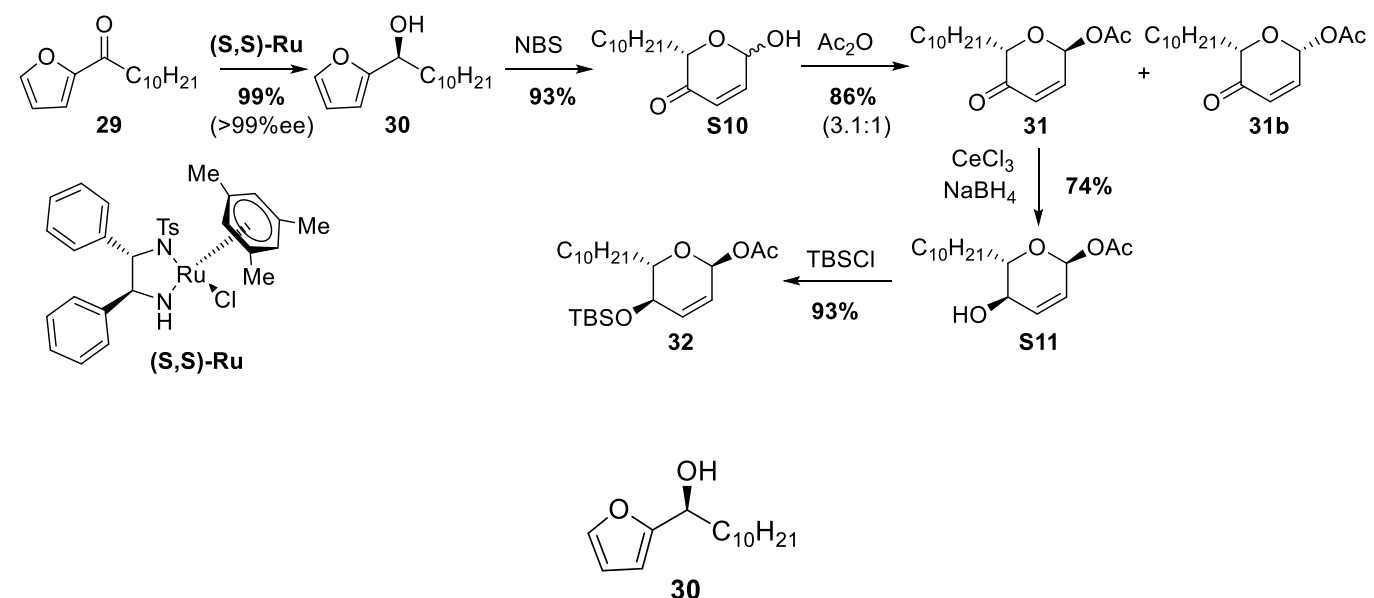

(S)-1-(furan-2-yl)undecan-1-olol (30) : To a solution of aqueous $\mathrm{HCO}_{2} \mathrm{Na}(4.96 \mathrm{~g}, 36 \mathrm{~mL}, 2.0 \mathrm{M})$ was added 1(furan-2-yl)undecan-1-one $\mathbf{2 9}^{6}(4.91 \mathrm{~g}, 20.8 \mathrm{~mol})$ and $\mathrm{CH}_{2} \mathrm{Cl}_{2}(2 \mathrm{~mL})$, followed by the addition of surfactant Cetyltrimethylammonium Bromide $(758 \mathrm{mg}, 2.08 \mathrm{mmol})$. The mixture was stirred for $5 \mathrm{~min}$, then added (S,S)Ru (129 mg, $1 \mathrm{~mol} \%$ ). The resulting solution was stirred at room temperature under argon for $22 \mathrm{~h}$. Then the mixture was added with sat. $\mathrm{NaHCO}_{3}(100 \mathrm{~mL})$ to dilute and extracted with EtOAc $(4 \times 50 \mathrm{~mL})$. The combined organic layer was washed with brine, dried over $\mathrm{Na}_{2} \mathrm{SO}_{4}$ and then concentrated under reduced pressure. The crude residue was purified with flashcolumn chromatography (Hexane:EtOAc $=90: 10$ ) to afford alcohol 30 as white solid (4.88 g, $20.5 \mathrm{mmol}, 99 \%$ yield).

$\mathrm{R}_{f} 0.25$ (EtOAc:Hex = 10:90); m.p 47-48 ${ }^{\circ} \mathrm{C}$; $[\alpha]^{16} \mathrm{D}-3.39\left(\mathrm{c}=0.73 \mathrm{CH}_{2} \mathrm{Cl}_{2}\right) ;{ }^{1} \mathrm{H} \mathrm{NMR}\left(500 \mathrm{MHz}, \mathrm{CDCl}_{3}\right) \delta 7.36-$ $7.37(\mathrm{~m}, 1 \mathrm{H}), 6.32(\mathrm{dd}, J=1.90,3.15 \mathrm{~Hz}, 1 \mathrm{H}), 6.23(\mathrm{~d}, J=3.15 \mathrm{~Hz}, 1 \mathrm{H}), 4.65-4.69(\mathrm{~m}, 1 \mathrm{H}), 1.83-1.88(\mathrm{~m}, 3 \mathrm{H})$, $1.39-1.46(\mathrm{~m}, 1 \mathrm{H}), 1.25-1.31(\mathrm{~m}, 15 \mathrm{H}), 0.88(\mathrm{~d}, J=6.70 \mathrm{~Hz}, 3 \mathrm{H}) \cdot ;{ }^{13} \mathrm{C}$ NMR $\left(125 \mathrm{MHz}, \mathrm{CDCl}_{3}\right) \delta 157.1,142.1$, 
110.3, 105.9, 68.1, 35.8, 32.1, 29.80, 29.78, 29.73, 29.6, 29.5, 25.7, 22.9, 14.3.; IR (NaCl) v 3255, 2955, 2916, 2849, 1506, 1466, 1318, 1224, 1192, 1148, 1114, $1082 \mathrm{~cm}^{-1}$; HRMS (ESI) m/z: $[\mathrm{M}+\mathrm{Na}]^{+}$Calcd for $\mathrm{C}_{15} \mathrm{H}_{26} \mathrm{O}_{2} \mathrm{Na}$ 261.1825; Found 261.1827.

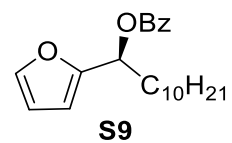

(S)-1-(furan-2-yl)undecyl benzoate (S9) : To a solution of compound $\mathbf{3 0}(8.7 \mathrm{mg}, 0.037 \mathrm{mmol})$ in $\mathrm{CH}_{2} \mathrm{Cl}_{2}(0.7$ $\mathrm{mL}), \mathrm{BzCl}(8.5 \mu \mathrm{L}, 0.073 \mathrm{mmol}), \mathrm{Et}_{3} \mathrm{~N}(10 \mu \mathrm{L}, 0.073 \mathrm{mmol})$ and DMAP $(0.4 \mathrm{mg}, 0.0037 \mathrm{mmol})$ was added at $0^{\circ} \mathrm{C}$. After stirred at room temperature for $4 \mathrm{~h}$. the reaction was quenched with distilled water then, extracted with $\mathrm{CH}_{2} \mathrm{Cl}_{2}(3 \times 20 \mathrm{~mL})$, dried over $\mathrm{Na}_{2} \mathrm{SO}_{4}$ and concentrated under reduced pressure. The crude mixture was purified by flash column chromatography (Hexane:EtOAc $=98: 2)$ to afford compound $\mathbf{S 9}$ as a colorless oil $(12.5 \mathrm{mg}$, $0.037 \mathrm{mmol}, 99 \%$ yield). The enantiomeric excess ( $>99 \%$ ee) was determined by HPLC on a chiral column (Chiralpak ID, Hexane:EtOAc $=99: 1$; flow rate $=0.5 \mathrm{~mL} / \mathrm{min} ; \mathrm{UV}=254 \mathrm{~nm})$; retention times $=11.83(100 \%)$. $\mathrm{R}_{f} 0.52$ (EtOAc:Hex = 10:90); $[\alpha]^{16} \mathrm{D}-39.83\left(\mathrm{c}=0.60 \mathrm{CH}_{2} \mathrm{Cl}_{2}\right) ;{ }^{1} \mathrm{H}$ NMR $\left(500 \mathrm{MHz}, \mathrm{CDCl}_{3}\right) \delta 8.05-8.06(\mathrm{~m}, 2 \mathrm{H})$, 7.41-7.44 (m, 2H), 7.38-7.39 (m, 1H), 6.39 (d, $J=3.23 \mathrm{~Hz}, 1 \mathrm{H}), 6.34$ (dd, $J=1.84,3.23 \mathrm{~Hz}, 1 \mathrm{H}), 6.08$ (t, $J=7.20$ $\mathrm{Hz}, 1 \mathrm{H}), 2.02-2.14(\mathrm{~m}, 2 \mathrm{H}), 1.24-1.35(\mathrm{~m}, 16 \mathrm{H}), 0.87(\mathrm{~d}, J=6.84 \mathrm{~Hz}, 3 \mathrm{H}) . ;{ }^{13} \mathrm{C}$ NMR $\left(125 \mathrm{MHz}, \mathrm{CDCl}_{3}\right) \delta 166.1$, 153.1, 142.6, 133.1, 130.5, 129.9, 128.5, 110.4, 108.7, 69.6, 32.9, 32.1, 29.77, 29.73, 29.6, 29.52, 29.46, 25.6, 22.9, 14.3.; IR $(\mathrm{NaCl}) \vee 2926,2855,1720,1602,1503,1452,1339,1315,1267,1176,1107 \mathrm{~cm}^{-1}$; HRMS (ESI) $\mathrm{m} / \mathrm{z}:[\mathrm{M}+\mathrm{Na}]^{+}$Calcd for $\mathrm{C}_{22} \mathrm{H}_{30} \mathrm{O}_{3} \mathrm{Na}$ 365.2087; Found 365.2085.

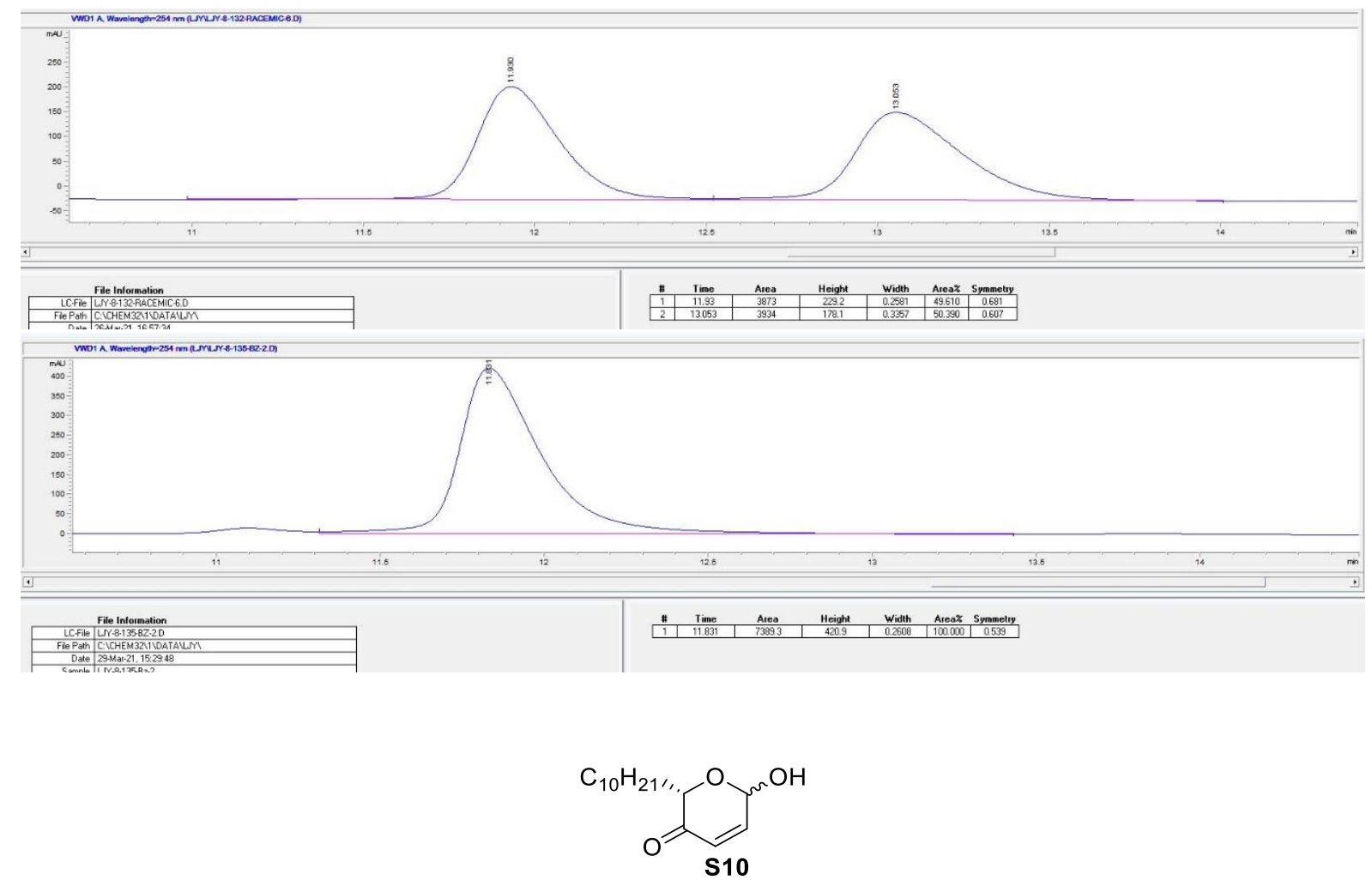

(2S)-2-decyl-6-hydroxy-2H-pyran-3(6H)-one (S10) : To a solution furan alcohol 30 (4.88 g, $20.5 \mathrm{mmol})$ in $\mathrm{THF} / \mathrm{H}_{2} \mathrm{O}(0.25 \mathrm{M}, \mathrm{v} / \mathrm{v}=3 / 1)$ was added $\mathrm{NaHCO}_{3}(3.44 \mathrm{~g}, 41 \mathrm{mmol}), \mathrm{NaOAc}(1.68 \mathrm{~g}, 20.5 \mathrm{mmol})$ and NBS (3.65 $\mathrm{g}, 20.5 \mathrm{mmol})$ at $0^{\circ} \mathrm{C}$. The reaction mixture was stirring at $0^{\circ} \mathrm{C}$ for $0.5 \mathrm{~h}$, then the reaction mixture was quenched with sat. $\mathrm{NaHCO}_{3}$ and extracted with EtOAc $(3 \times 50 \mathrm{~mL})$, washed with brine, dried over $\mathrm{Na}_{2} \mathrm{SO}_{4}$ and concentrated reduced pressure. The crude mixture was purified by flash column chromatography (Hexane:EtOAc $=80: 20)$ to afford compound $\mathbf{S 1 0}$ as a white solid $(4.87 \mathrm{~g}, 19.2 \mathrm{mmol}, 93 \%$ yield). 
$\mathrm{R}_{f} 0.20$ (EtOAc:Hex = 20:80); m.p 68-69 ${ }^{\circ} \mathrm{C}$; $[\alpha]^{16}{ }_{\mathrm{D}}+19.30\left(\mathrm{c}=0.93 \mathrm{CH}_{2} \mathrm{Cl}_{2}\right) ;{ }^{1} \mathrm{H}$ NMR $\left(500 \mathrm{MHz}, \mathrm{CDCl}_{3}\right) \delta 6.91$ $\left(\mathrm{dd}, J=1.41,10.32 \mathrm{~Hz}, 1 \mathrm{H}_{\mathrm{b}}\right), 6.89(\mathrm{dd}, J=3.33,10.20 \mathrm{~Hz}, 1 \mathrm{Ha}), 6.14\left(\mathrm{~d}, J=10.32 \mathrm{~Hz}, 1 \mathrm{H}_{\mathrm{b}}\right), 6.10(\mathrm{~d}, J=10.20$ $\left.\mathrm{Hz}, 1 \mathrm{H}_{\mathrm{a}}\right), 5.65\left(\mathrm{brs}, 1 \mathrm{H}_{\mathrm{a}} 1 \mathrm{H}_{\mathrm{b}}\right), 4.55\left(\mathrm{dd}, J=3.89,8.19 \mathrm{~Hz}, 1 \mathrm{H}_{\mathrm{a}}\right), 4.07\left(\mathrm{dd}, J=3.88,8.27 \mathrm{~Hz}, 1 \mathrm{H}_{\mathrm{b}}\right), 3.41\left(\mathrm{brs}, 1 \mathrm{H}_{\mathrm{b}}\right)$, 3.15 (brs, $\left.1 \mathrm{H}_{\mathrm{a}}\right), 1.90-1.94\left(\mathrm{~m}, 1 \mathrm{H}_{\mathrm{a}} 1 \mathrm{H}_{\mathrm{b}}\right), 1.74-1.80\left(\mathrm{~m}, 1 \mathrm{H}_{\mathrm{b}}\right), 1.65-1.73\left(\mathrm{~m}, 1 \mathrm{H}_{\mathrm{a}} 1 \mathrm{H}_{\mathrm{b}}\right), 1.25-1.41\left(\mathrm{~m}, 16 \mathrm{H}_{\mathrm{a}} 15 \mathrm{H}_{\mathrm{b}}\right)$, $0.87\left(\mathrm{t}, J=6.54 \mathrm{~Hz}, 3 \mathrm{H}_{\mathrm{a}} 3 \mathrm{H}_{\mathrm{b}}\right) ;{ }^{13} \mathrm{C} \mathrm{NMR}\left(125 \mathrm{MHz}, \mathrm{CDCl}_{3}\right) \delta 196.9,196.5,147.8,144.5,129.0,127.9,91.1,87.9$, 79.2, 74.5, 32.1 , 29.9, 29.80, 29.79, 29.7, 29.64, 29.60, 29.5, 25.4, 25.2, 22.9, 14.3.; IR (NaCl) v 3323, 2917, 2947 , 2852, 1676, 1472, 1375, 1280, 1154, $1100 \mathrm{~cm}^{-1}$; HRMS (ESI) m/z: [M + Na $]^{+}$Calcd for $\mathrm{C}_{15} \mathrm{H}_{26} \mathrm{O}_{3} \mathrm{Na}_{277.1774}$; Found 277.1777. HRMS (ESI) calcd for $\mathrm{C}_{15} \mathrm{H}_{26} \mathrm{O}_{3} \mathrm{Na}\left(\mathrm{M}+\mathrm{Na}^{+}\right) 277.1774$, found 277.1777.

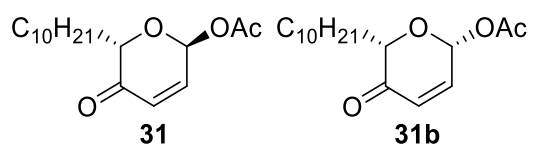

(2S,6S)-6-decyl-5-oxo-5,6-dihydro-2H-pyran-2-yl acetate (31) : As same procedure as described for 2 using compound S10 (2.54 g, $10 \mathrm{mmol})$, The crude oil was purified with flashcolumn chromatography (Hexane:EtOAc $=95: 5)$ to afford $\alpha$-product $\mathbf{3 1}$ as a white solid (1.93 g, $6.5 \mathrm{mmol}, 65 \%$ yield) and $\beta$-product $\mathbf{3 1 b}$ as a colorless oil (621 mg, $2.1 \mathrm{mmol}, 21 \%$ yield)

$\mathrm{R}_{f} 0.26$ (EtOAc:Hex = 10:90); m.p 36-37 ${ }^{\circ} \mathrm{C}$; $[\alpha]^{16}{ }_{\mathrm{D}}+79.51\left(\mathrm{c}=1.07 \mathrm{CH}_{2} \mathrm{Cl}_{2}\right) ;{ }^{1} \mathrm{H}$ NMR $\left(500 \mathrm{MHz}, \mathrm{CDCl}_{3}\right) \delta 6.87$ $(\mathrm{dd}, J=3.73,10.26 \mathrm{~Hz}, 1 \mathrm{H}), 6.49(\mathrm{~d}, J=3.73 \mathrm{~Hz}, 1 \mathrm{H}), 6.20(\mathrm{~d}, J=10.26 \mathrm{~Hz}, 1 \mathrm{H}), 4.45(\mathrm{dd}, J=3.86,7.63 \mathrm{~Hz}$, $1 \mathrm{H}), 2.12(\mathrm{~s}, 3 \mathrm{H}), 1.89-1.96(\mathrm{~m}, 1 \mathrm{H}), 1.68-1.75(\mathrm{~m}, 1 \mathrm{H}), 1.38-1.44(\mathrm{~m}, 2 \mathrm{H}), 1.25-1.29(\mathrm{~m}, 14 \mathrm{H}), 0.87(\mathrm{t}, J=6.78$ $\mathrm{Hz}, 3 \mathrm{H}) . ;{ }^{13} \mathrm{C}$ NMR $\left(125 \mathrm{MHz}, \mathrm{CDCl}_{3}\right) \delta 195.9,169.8,141.7,128.9,87.4,76.1,32.1,29.85,29.79,29.63,29.57$, 29.52, 24.8, 22.9, 21.1, 14.3.; IR (NaCl) v 2955, 2926, 2855, 2756, 1700, 1465, 1370, 1300, 1220, $1174 \mathrm{~cm}^{-1}$; HRMS (ESI) m/z: [M + Na] $]^{+}$Calcd for $\mathrm{C}_{17} \mathrm{H}_{28} \mathrm{O}_{4} \mathrm{Na} 319.1880$; Found 319.1879.

\section{(2S)-2-decyl-6-hydroxy-2H-pyran-3(6H)-one (31b) :}

$\mathrm{R}_{f} 0.21$ (EtOAc:Hex = 10:90); $[\alpha]^{17} \mathrm{D}-68.68\left(\mathrm{c}=1.48, \mathrm{CH}_{2} \mathrm{Cl}_{2}\right) ;{ }^{1} \mathrm{H}$ NMR $\left(500 \mathrm{MHz}, \mathrm{CDCl}_{3}\right) \delta 6.83(\mathrm{dd}, J=2.76$, $10.45 \mathrm{~Hz}, 1 \mathrm{H}), 6.53-6.54(\mathrm{~m}, 1 \mathrm{H}), 6.20(\mathrm{~d}, J=10.45 \mathrm{~Hz}, 1 \mathrm{H}), 4.20(\mathrm{dd}, J=5.31,8.55 \mathrm{~Hz}, 1 \mathrm{H}), 2.13(\mathrm{~s}, 3 \mathrm{H}), 1.80-$ 1.85 (m, 2H), 1.25-1.50 (m, 16H), 0.87 (t, $J=6.74 \mathrm{~Hz}, 3 \mathrm{H}) . ;{ }^{13} \mathrm{C}$ NMR (125 MHz, CDCl 3 ) $\delta 196.0,169.5,142.9$, 128.6, 87.8, 79.9, 33.2, 32.1, 29.8, 29.6, 29.50, 29.48, 25.5, 22.9, 21.2, 14.3.; IR (NaCl) v 2926, 2855, 1756, 1697, 1465, 1371, 1267, 1221, 1174, 1122, $1104 \mathrm{~cm}^{-1}$; HRMS (ESI) m/z: [M + Na] ${ }^{+}$Calcd for $\mathrm{C}_{17} \mathrm{H}_{28} \mathrm{O}_{4} \mathrm{Na} 319.1880$; Found 319.1881 .<smiles>CC(=O)O[C@@H]1C=C[C@@H](O)[C@@H]([18OH])O1</smiles>

(2S,5R,6S)-6-decyl-5-hydroxy-5,6-dihydro-2H-pyran-2-yl acetate (S11) : A solution of compound 31 (1.82 g, $6.1 \mathrm{mmol})$ in $\mathrm{CH}_{2} \mathrm{Cl}_{2}(30 \mathrm{~mL})$, added $\mathrm{CeCl}_{3}(3.46 \mathrm{~g}, 14 \mathrm{mmol})$ in $\mathrm{MeOH}(30 \mathrm{~mL})$ at $-78^{\circ} \mathrm{C}$. $\mathrm{NaBH}_{4}(346 \mathrm{mg}, 9.2$ mmol) was added and the resulting solution was gradually allowed to warm at $-40^{\circ} \mathrm{C}$ over $1 \mathrm{~h}$. The reaction mixture was diluted with $\mathrm{Et}_{2} \mathrm{O}(20 \mathrm{~mL})$ and was quenched with sat. $\mathrm{NaHCO}_{3}$, extracted with $\mathrm{Et}_{2} \mathrm{O}(5 \times 30 \mathrm{~mL})$, dried with $\mathrm{Na}_{2} \mathrm{SO}_{4}$ and concentrated under reduced pressure. The crude mixture was purified with flashcolumn chromatography (Hexane:EtOAc $=85: 15)$ to product $\mathbf{S 1 1}$ as a colorless oil (1.34 g, $4.5 \mathrm{mmol}$, 74\% yield). $\mathrm{R}_{f} 0.27$ (EtOAc:Hex = 20:80); $[\alpha]^{17} \mathrm{D}-27.60\left(\mathrm{c}=0.89, \mathrm{CH}_{2} \mathrm{Cl}_{2}\right) ;{ }^{1} \mathrm{H}$ NMR $\left(300 \mathrm{MHz}, \mathrm{CDCl}_{3}\right) \delta 6.21-6.22(\mathrm{~m}, 1 \mathrm{H})$, 6.03-6.07 (m, 1H), 5.74-5.79 (m, 1H), 3.94-3.99 (m, 1H), 3.54-3.60 (m, 1H), $2.08(\mathrm{~s}, 3 \mathrm{H}), 1.78-1.86(\mathrm{~m}, 1 \mathrm{H})$, 1.61-1.64 (m, 1H), 1.42-1.53 (m, 2H), 1.26-1.37 (m, 15H), $0.87(\mathrm{t}, J=6.51 \mathrm{~Hz}, 3 \mathrm{H}) .{ }^{13} \mathrm{C} \mathrm{NMR}\left(75 \mathrm{MHz}, \mathrm{CDCl}_{3}\right)$ $\delta 170.3,135.1,125.0,88.7,74.0,67.7,32.1,31.7,29.9,29.82,29.76,29.6,29.5,25.3,22.9,21.4,14.3 . ; \mathrm{IR}(\mathrm{NaCl})$ $v$ 3450, 3047, 2925, 2855, 1750, 1465, 1435, 1372, 1234, 1191, 1144, $1121 \mathrm{~cm}^{-1}$; HRMS (ESI) m/z: [M + Na $]^{+}$ Calcd for $\mathrm{C}_{17} \mathrm{H}_{30} \mathrm{O}_{4} \mathrm{Na} 321.2036$; Found 321.2035.

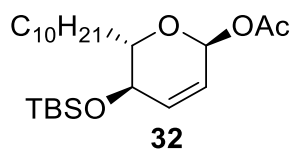


(2S,5R,6S)-5-((tert-butyldimethylsilyl)oxy)-6-decyl-5,6-dihydro-2H-pyran-2-yl acetate (32) : As same procedure as described for $\mathbf{S 1}$ using compound $\mathbf{S 1 1}$ (1.34 g, $4.5 \mathrm{mmol})$, The crude mixture was purified with flashcolumn chromatography (Hexane: $\left.\mathrm{Et}_{2} \mathrm{O}=98: 2\right)$ to give desired product $32(1.67 \mathrm{~g}, 4.05 \mathrm{mmol}, 93 \%$ yield $)$ as a colorless oil.

$\mathrm{R}_{f} 0.47$ (EtOAc:Hex = 10:90); $[\alpha]^{17} \mathrm{D}-62.26\left(\mathrm{c}=1.11, \mathrm{CH}_{2} \mathrm{Cl}_{2}\right) ;{ }^{1} \mathrm{H}$ NMR $\left(300 \mathrm{MHz}, \mathrm{CDCl}_{3}\right) \delta 6.20-6.21(\mathrm{~m}, 1 \mathrm{H})$, 5.94-5.98 (m, 1H), 5.69 (ddd, $J=2.04,2.81,10.19 \mathrm{~Hz}, 1 \mathrm{H}), 3.94-3.98(\mathrm{~m}, 1 \mathrm{H}), 3.57-3.63(\mathrm{~m}, 1 \mathrm{H}), 2.07(\mathrm{~s}, 3 \mathrm{H})$, $1.71-1.85(\mathrm{~m}, 1 \mathrm{H}), 1.29-1.47(\mathrm{~m}, 17 \mathrm{H}), 0.85-0.91(\mathrm{~m}, 12 \mathrm{H}), 0.10(\mathrm{~s}, 3 \mathrm{H}), 0.09(\mathrm{~s}, 3 \mathrm{H}) . ;{ }^{13} \mathrm{C} \mathrm{NMR}\left(75 \mathrm{MHz} \mathrm{CDCl}_{3}\right)$ $\delta 170.4,136.5,123.9,89.0,73.7,68.2,32.1,31.4,29.86,29.83,29.7,29.54,29.48,25.9,25.1,22.9,21.4,18.2$, 14.3, -3.96, -4.50; IR (NaCl) v 2955, 2928, 2857, 1752, 1466, 1369, 1310, 1253, 1231, 1192, 1148, $1121 \mathrm{~cm}^{-1}$; HRMS (ESI) m/z: $[\mathrm{M}+\mathrm{Na}]^{+}$Calcd for $\mathrm{C}_{23} \mathrm{H}_{44} \mathrm{O}_{4} \mathrm{SiNa} 435.2901$; Found 435.2905.

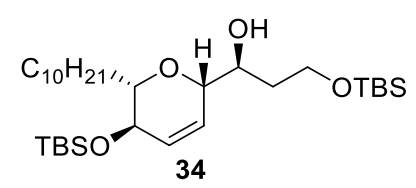

(S)-3-((tert-butyldimethylsilyl)oxy)-1-((2S,5R,6S)-5-((tert-butyldimethylsilyl)oxy)-6-decyl-5,6-dihydro-2Hpyran-2-yl)propan-1-ol (34) : Following the general procedure using 3-((tert-butyldimethylsilyl)oxy)propanal $33(564 \mathrm{mg}, 3.0 \mathrm{mmol})$ and acetate $32(412 \mathrm{mg}, 1.0 \mathrm{mmol})$, Flash column chromatography (EtOAc:Hexane = 4:96) afforded the compound $\mathbf{3 4}$ as a colorless oil ( $380 \mathrm{mg}, 0.70 \mathrm{mmol}, 70 \%$ yield).

$\mathrm{R}_{f} 0.49$ (EtOAc:Hex = 10:90); $[\alpha]^{17} \mathrm{D}-79.74\left(\mathrm{c}=0.58, \mathrm{CH}_{2} \mathrm{Cl}_{2}\right) ;{ }^{1} \mathrm{H}$ NMR $\left(500 \mathrm{MHz}, \mathrm{CDCl}_{3}\right) \delta 5.77(\mathrm{~s}, 2 \mathrm{H}), 3.99-$ $4.03(\mathrm{~m}, 1 \mathrm{H}), 3.92-3.94(\mathrm{~m}, 1 \mathrm{H}), 3.74-3.88(\mathrm{~m}, 3 \mathrm{H}), 3.22-3.26(\mathrm{~m}, 1 \mathrm{H}), 2.97(\mathrm{~d}, J=3.55 \mathrm{~Hz}, 1 \mathrm{H}), 1.69-1.81(\mathrm{~m}$, $3 \mathrm{H}), 1.50-1.52(\mathrm{~m}, 1 \mathrm{H}), 1.26-1.39(\mathrm{~m}, 19 \mathrm{H}), 0.90(\mathrm{~s}, 9 \mathrm{H}), 0.89(\mathrm{~s}, 9 \mathrm{H}), 0.093(\mathrm{~s}, 3 \mathrm{H}), 0.079(\mathrm{~s}, 3 \mathrm{H}), 0.066(\mathrm{~s}, 6 \mathrm{H})$; ${ }^{13} \mathrm{C} \mathrm{NMR}\left(125 \mathrm{MHz}, \mathrm{CDCl}_{3}\right) \delta 132.3,127.8,79.3,77.7,72.5,68.8,61.8,35.0,32.3,32.1,29.9,29.79,29.78,29.6$, 26.1, 26.0, 25.6, 22.9, 18.5, 18.2, 14.3, -3.87, -4.42, -5.22, -5.26.; IR (NaCl) v 3510, 3040, 2955, 2925, 2857, 1469, 1388, 1361, 1313, 1090, 1006, $970 \mathrm{~cm}^{-1}$; HRMS (ESI) m/z: $[\mathrm{M}+\mathrm{Na}]^{+}$Calcd for $\mathrm{C}_{30} \mathrm{H}_{62} \mathrm{O}_{4} \mathrm{Si}_{2} \mathrm{Na}_{565.4079}$; Found 565.4080 .

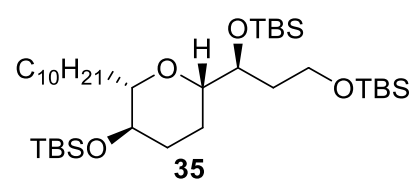

(S)-5-((2S,5R,6S)-5-((tert-butyldimethylsilyl)oxy)-6-decyltetrahydro-2H-pyran-2-yl)-2,2,3,3,9,9,10,10octamethyl-4,8-dioxa-3,9-disilaundecane (35) : To a solution of compound 34 in EtOAc $(2 \mathrm{~mL})$, added $10 \%$ $\mathrm{Pd} / \mathrm{C}(21.6 \mathrm{mg}, 20 \mathrm{wt} \%)$ at room temperature. The mixture was stirred under $\mathrm{H}_{2}$ atmosphere $(1 \mathrm{~atm})$ for $2 \mathrm{~h}$. Then the solution was filtered through a pad of celite (rines; $\mathrm{CH}_{2} \mathrm{Cl}_{2}$ ) and concentrated under reduced pressure. Without purification, the residue was dissolved in $\mathrm{CH}_{2} \mathrm{Cl}_{2}(2 \mathrm{~mL})$ and reacted with 2,6-Lutidine $(0.11 \mathrm{~mL}, 1.0 \mathrm{mmol})$ and TBDMS-OTf $(0.23 \mathrm{~mL}, 0.6 \mathrm{mmol})$ at $0^{\circ} \mathrm{C}$. And the reaction mixture was stirred at same temperature for $1 \mathrm{~h}$. The reaction mixture was quenched with a sat. $\mathrm{NaHCO}_{3}$, extracted with $\mathrm{Et}_{2} \mathrm{O}(4 \times 25 \mathrm{~mL})$, washed with brine, dried over $\mathrm{Na}_{2} \mathrm{SO}_{4}$ and concentrated under reduced pressure. The crude mixture was purified with flashcolumn chromatography (Hexane: $\left.\mathrm{CH}_{2} \mathrm{Cl}_{2}=80: 20\right)$ to give desired product 35 (92 $\mathrm{mg}, 0.14 \mathrm{mmol}, 70 \%$ yield) as a colorless oil.

$\mathrm{R}_{f} 0.28\left(\mathrm{CH}_{2} \mathrm{Cl}_{2}: \mathrm{Hex}=10: 80\right) ;[\alpha]^{21}{ }_{\mathrm{D}}-38.57\left(\mathrm{c}=0.98, \mathrm{CHCl}_{3}\right) ;{ }^{1} \mathrm{H}$ NMR $\left(500 \mathrm{MHz}, \mathrm{CDCl}_{3}\right) \delta 3.75-3.79(\mathrm{~m}, 1 \mathrm{H})$, 3.61-3.70 (m, 2H), 3.19-3.23 (m, 2H), 2.97-3.01 (m, 1H), 1.93-2.02 (m, 1H), 1.63-1.80 (m, 3H), 1.50-1.56 (m, $1 \mathrm{H}), 1.24-1.43(\mathrm{~m}, 19 \mathrm{H}), 0.86-0.87(\mathrm{~m}, 30 \mathrm{H}), 0.02-0.03(\mathrm{~m}, 18 \mathrm{H}) \cdot ;{ }^{13} \mathrm{C} \mathrm{NMR}\left(125 \mathrm{MHz}, \mathrm{CDCl}_{3}\right) \delta 82.3,79.8$, $71.1,70.9,60.1,35.5,33.5,32.0,31.9,29.8,29.68,29.66,29.3,25.99,25.96,25.92,25.8,25.1,25.0,22.7,18.3$, $18.2,18.0,14.1,-4.0,-4.4,-4.74,-4.76,-5.22,-5.29$; IR (NaCl) v 2955, 2928, 2857, 1470, 1388, 1361, 1254, 1218, 1187, 1096, 1006, 938, $873 \mathrm{~cm}^{-1}$; HRMS (ESI) m/z: [M + Na] ${ }^{+}$Calcd for $\mathrm{C}_{36} \mathrm{H}_{78} \mathrm{O}_{4} \mathrm{Si}_{3} \mathrm{Na}_{681.5100}$; Found 681.5107. 
6. Determination of Absolute configuration of compound 3a, 22, 24 and 34

\section{6-1. compound 2a}

\section{- NOESY}
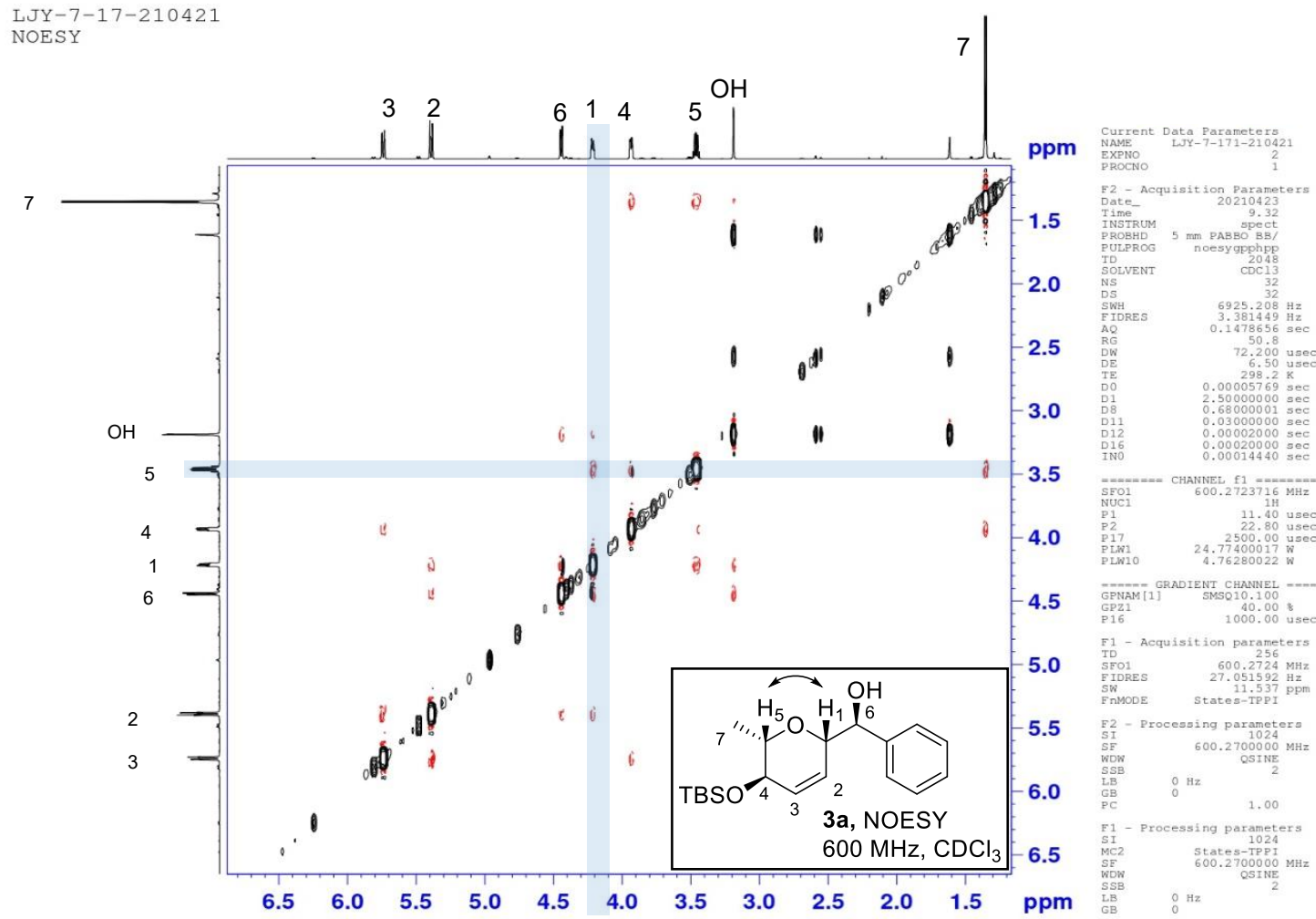


\section{-Mosher's method}
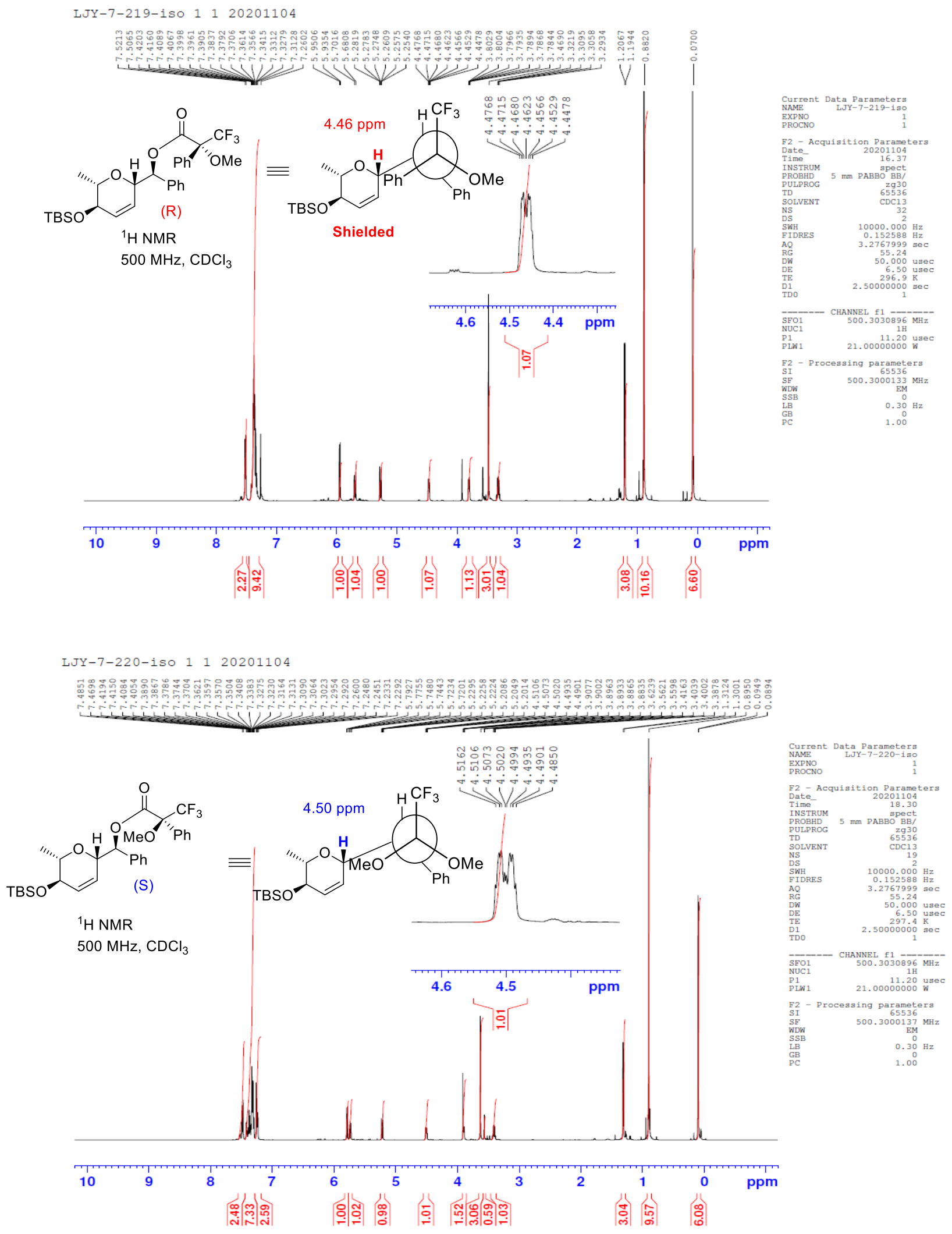
- Oxidation of alcohol

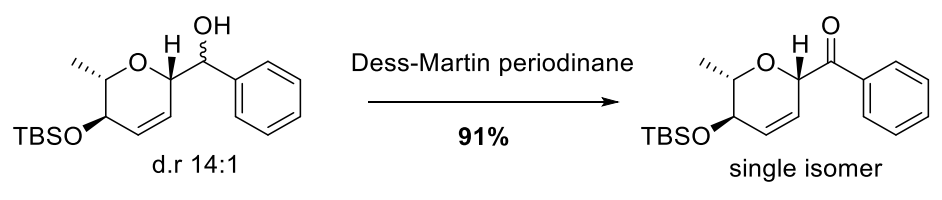

$$
\text { LUY }-7-17-210421
$$

1H

-

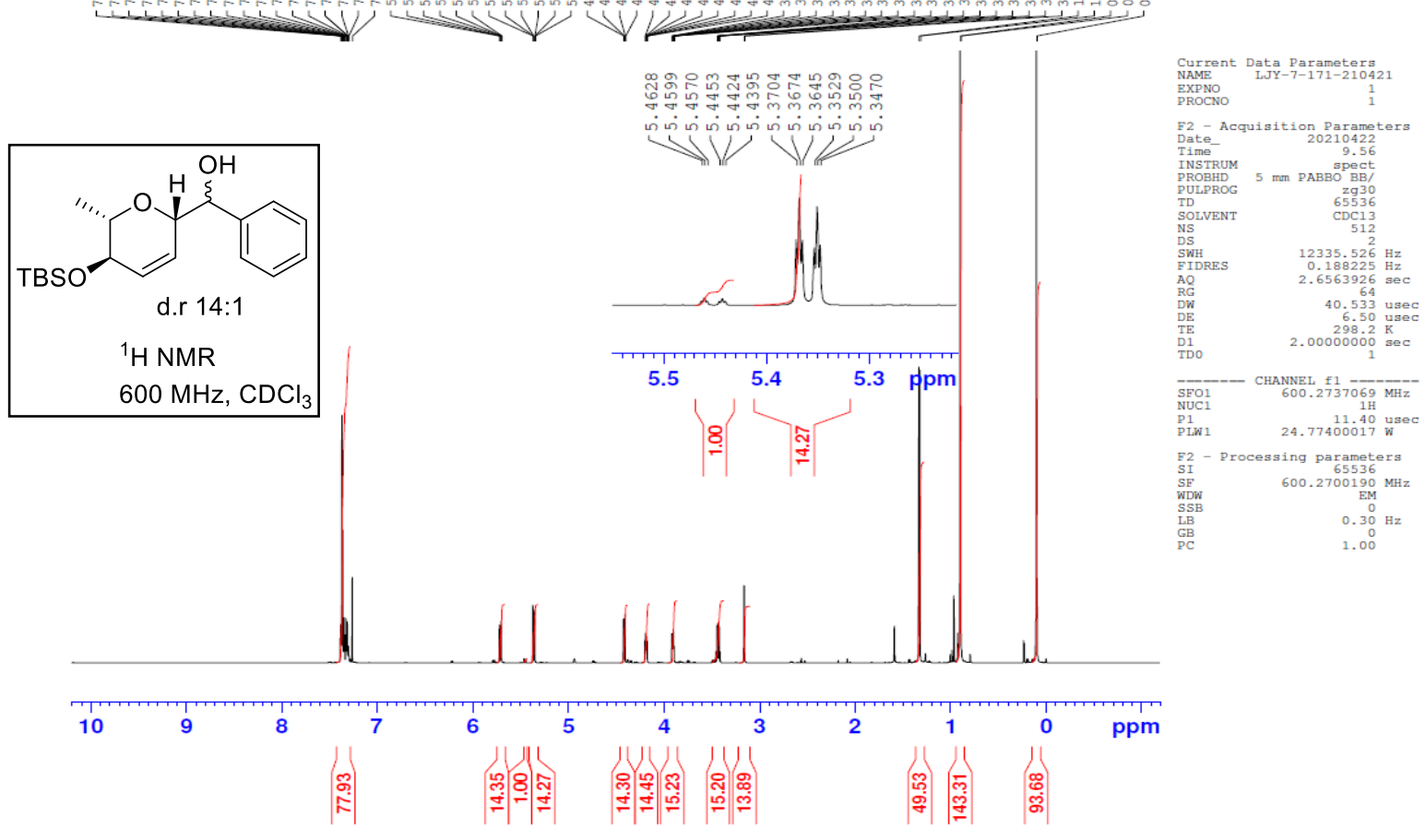

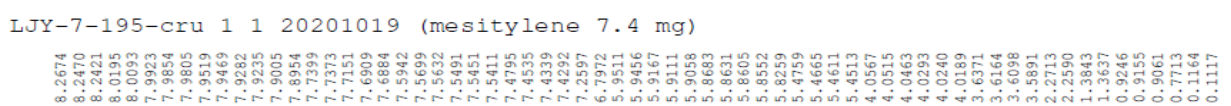

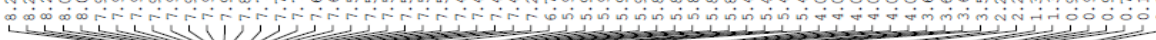
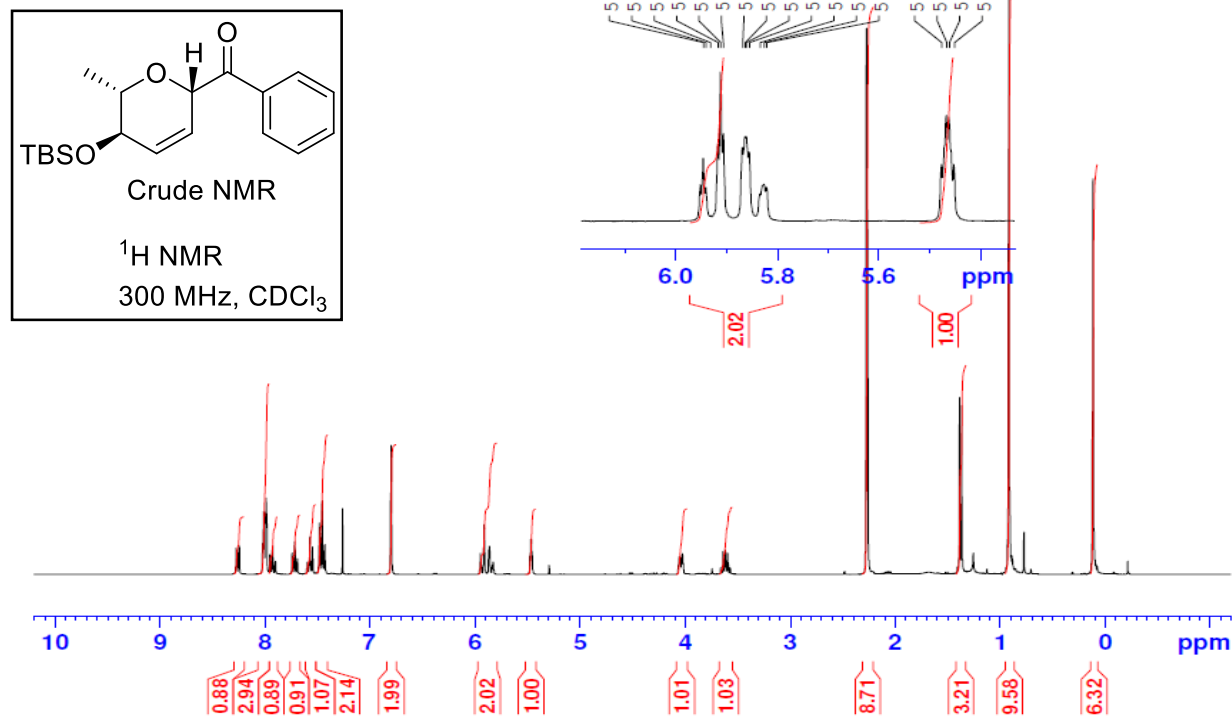

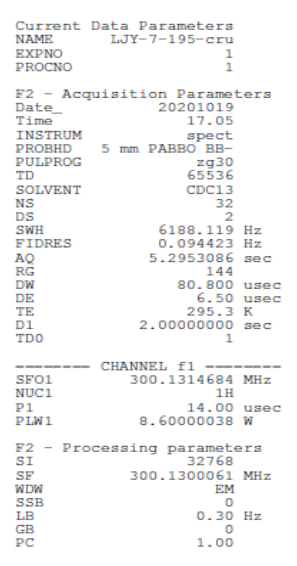




\section{6-2. compound 22}

\section{- NOESY}

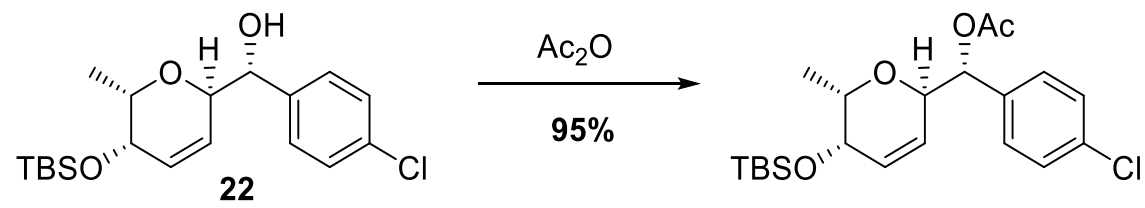

Due to peak separation in NMR, stereoselectivity of $\mathbf{2 2}$ was confirmed after acetylation

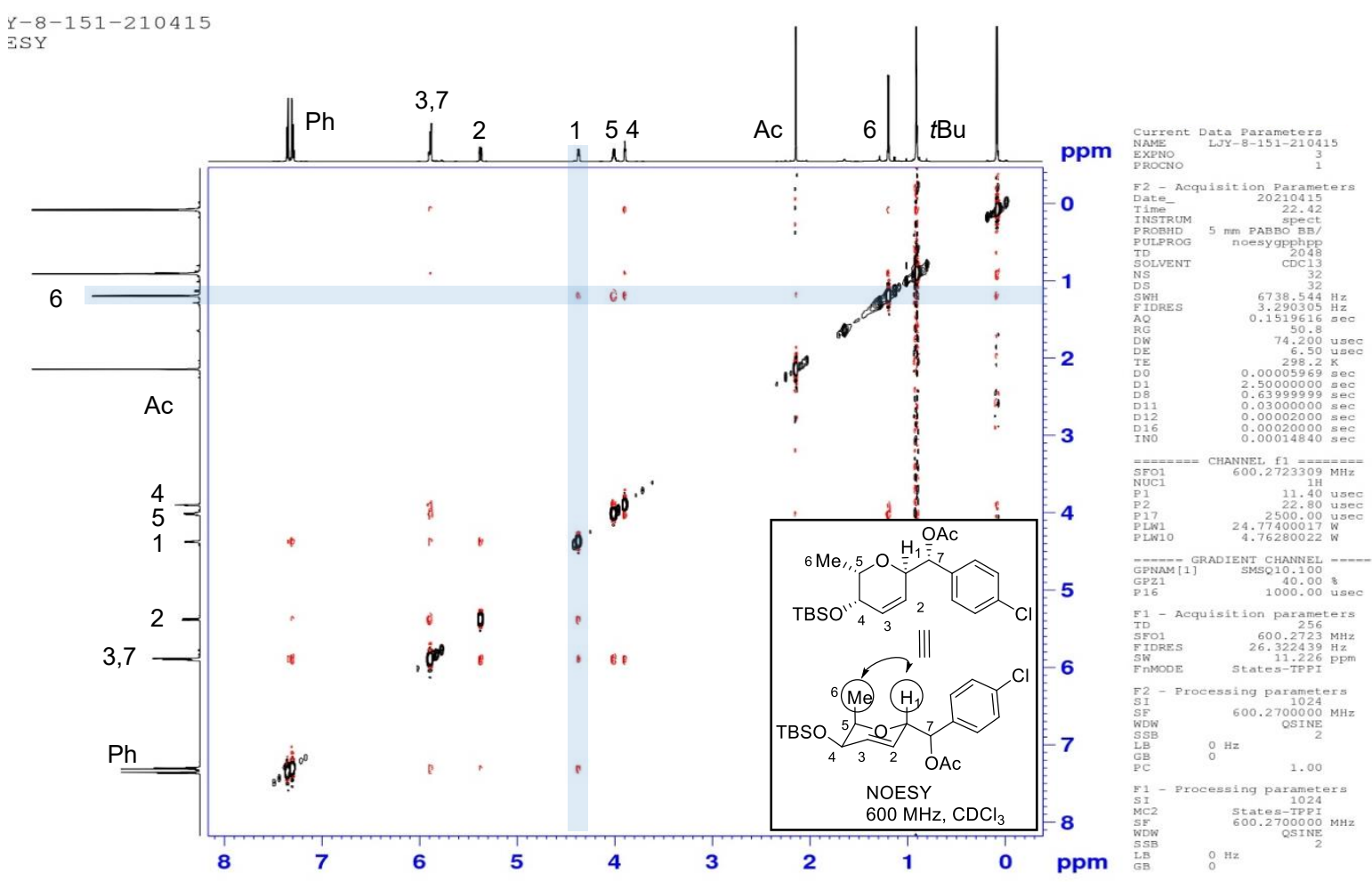




\section{6-3. compound 24}

\section{- NOESY}

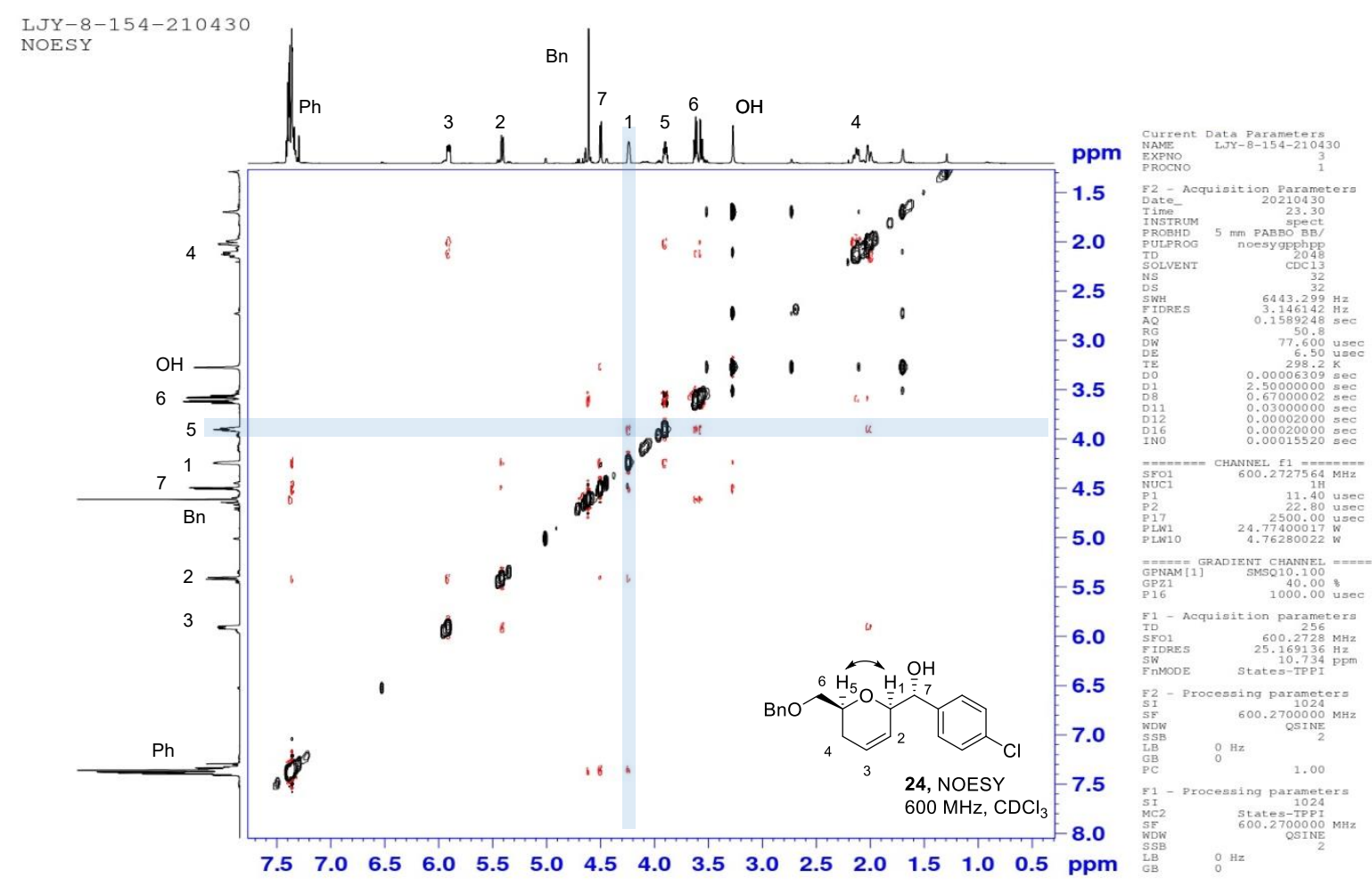




\section{- Oxidation of alcohol}<smiles>OC(c1ccccc1)C1C=CCC(COCc2ccccc2)O1</smiles>

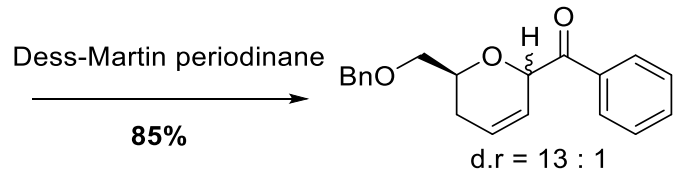

iso 1 : iso 2 : iso $3=12 \cdot 2: 1.4: 1$

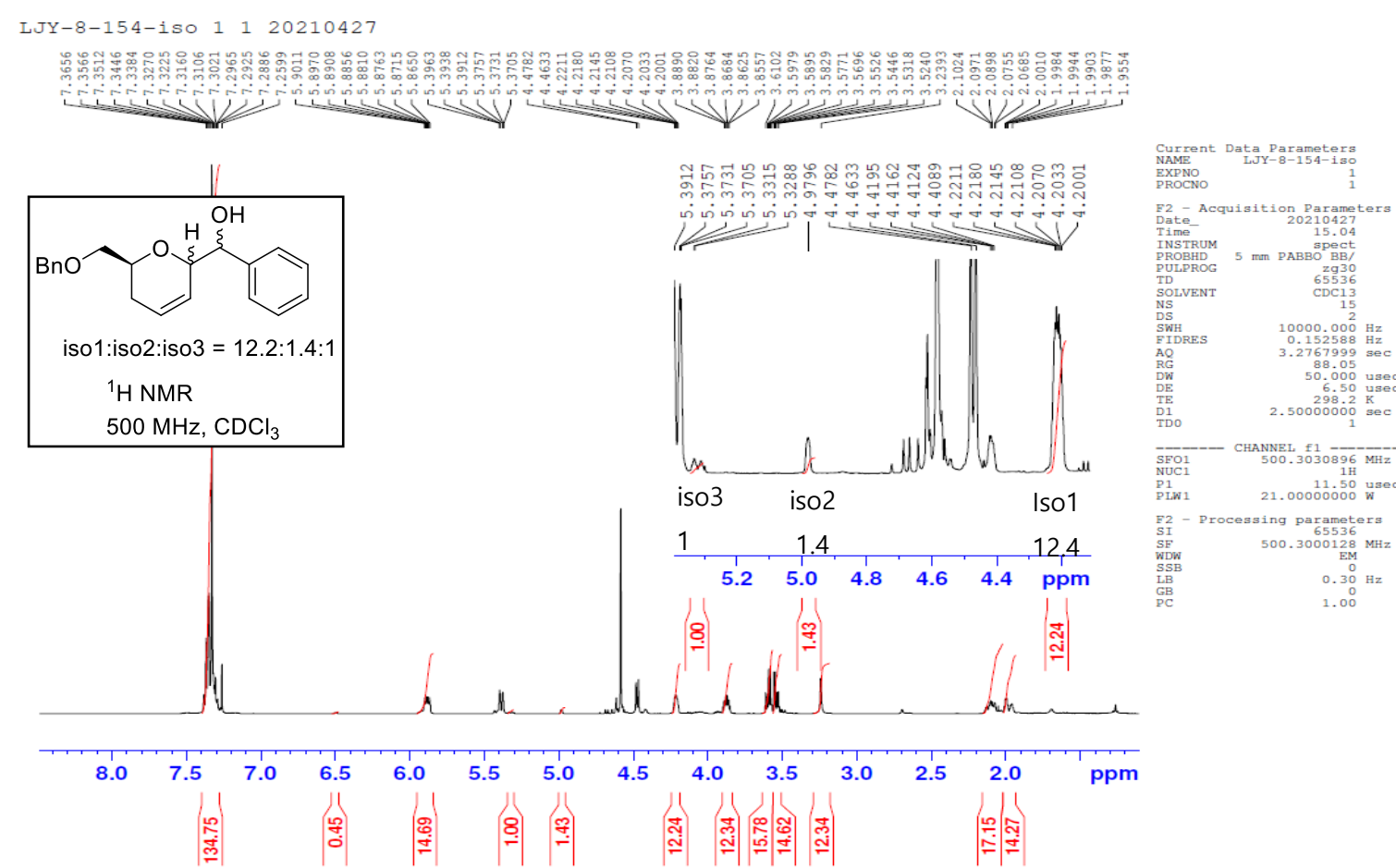

LJY-7-156-cru 1120210428 (mesitylene $6.5 \mathrm{mg}$ )

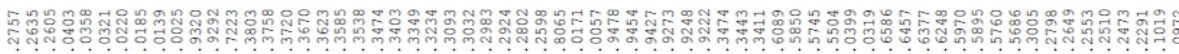

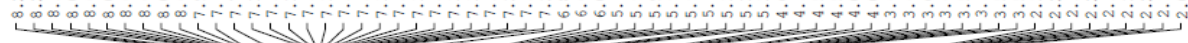
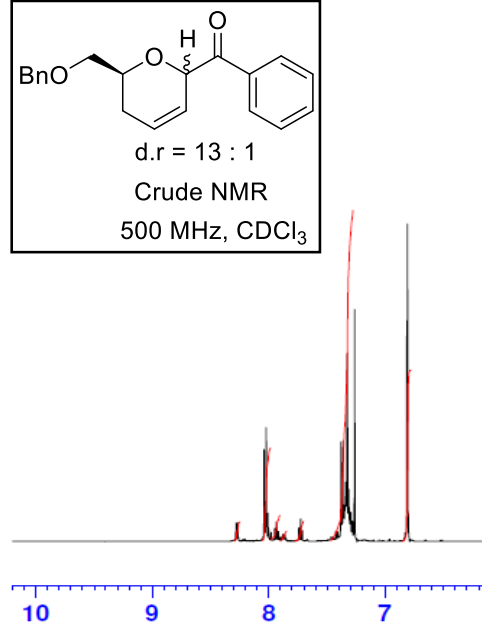$$
8
$$

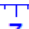

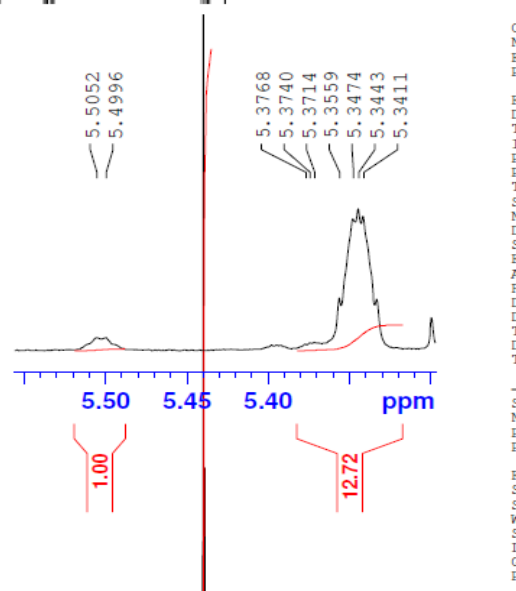

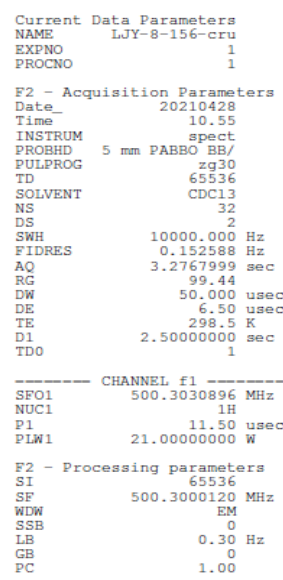

H.
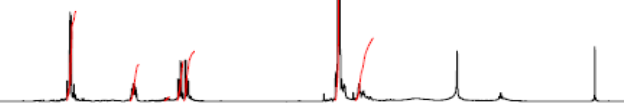


\section{6-4. compound 34}

\section{- NOESY}

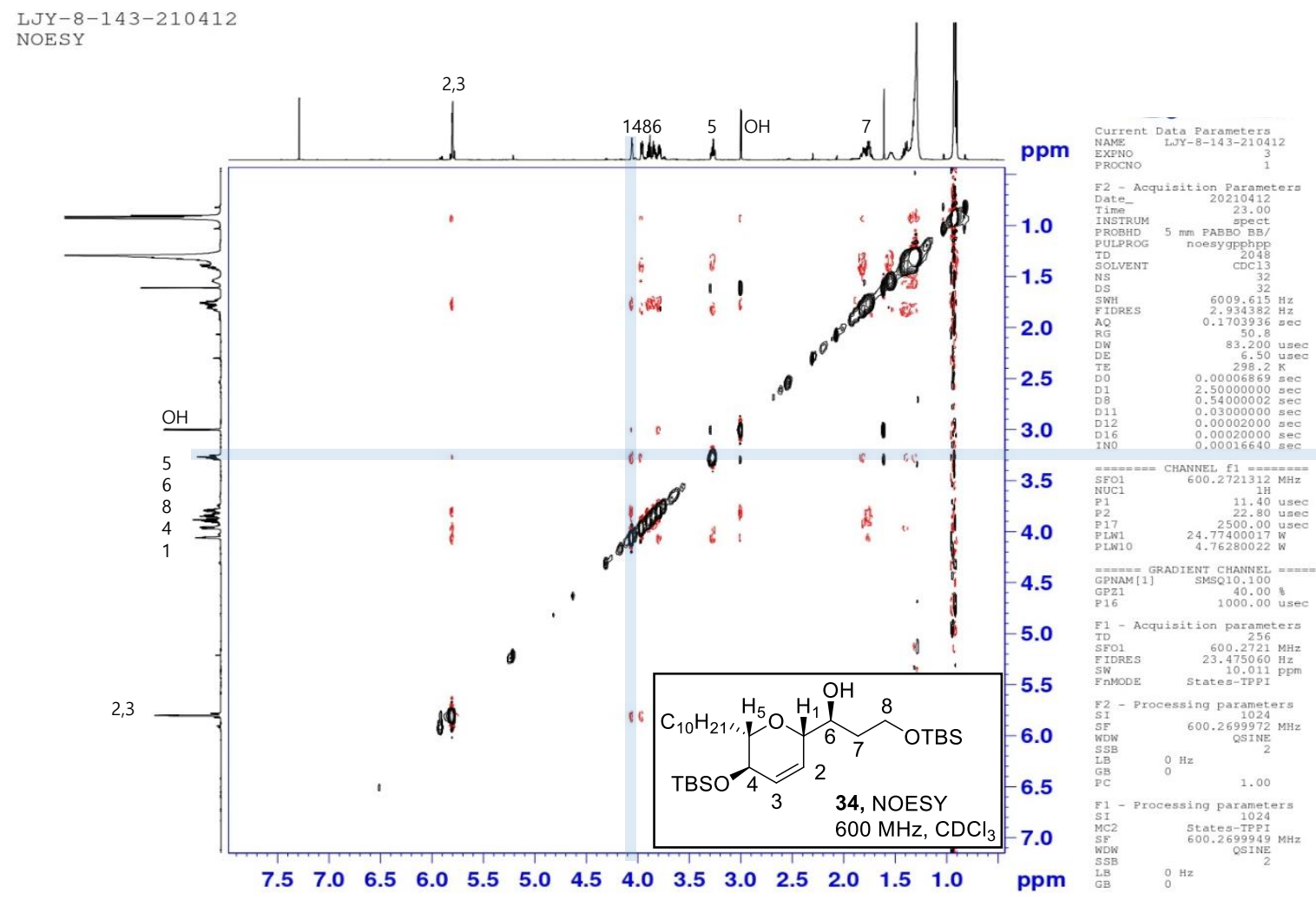




\section{- Mosher'smethod}

LJY-8-146-iso-R 120210405

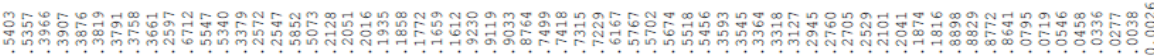

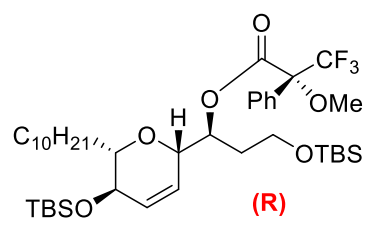

${ }^{1} \mathrm{H}$ NMR

$500 \mathrm{MHz}, \mathrm{CDCl}_{3}$

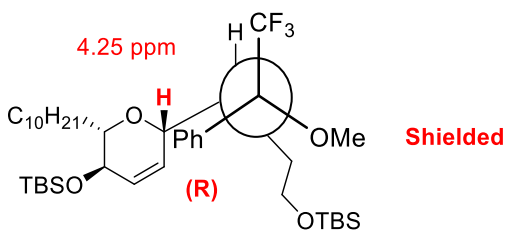

di
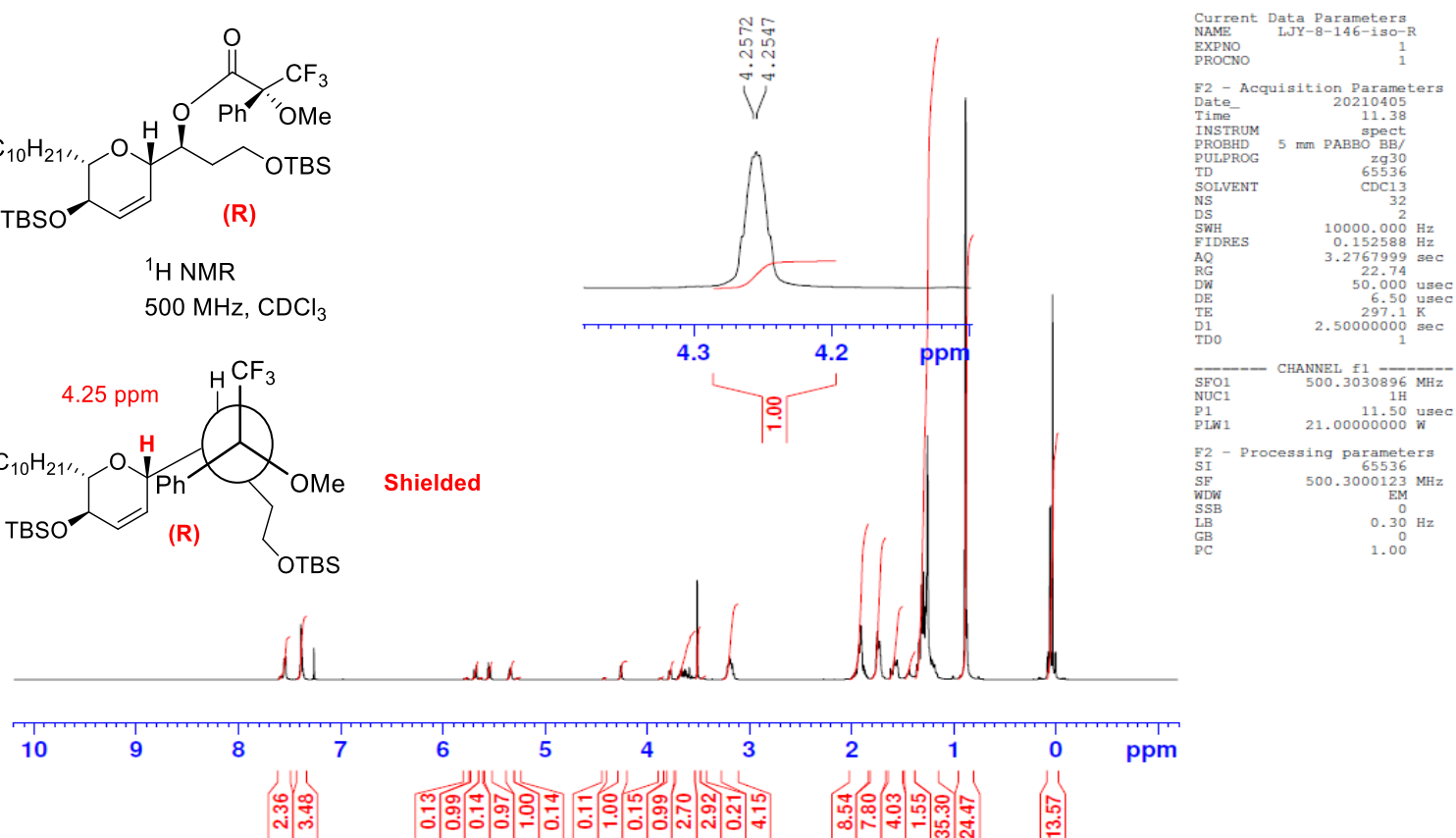

LJY-8-146-iso-S 120210405

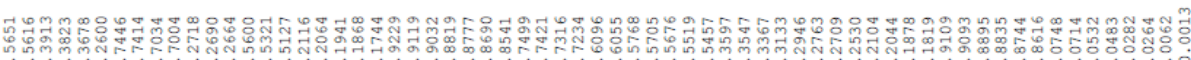

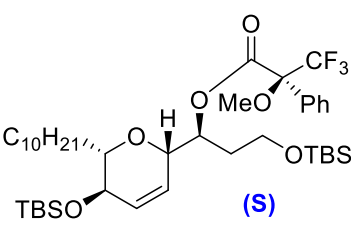

${ }^{1} \mathrm{H}$ NMR

$500 \mathrm{MHz}, \mathrm{CDCl}_{3}$

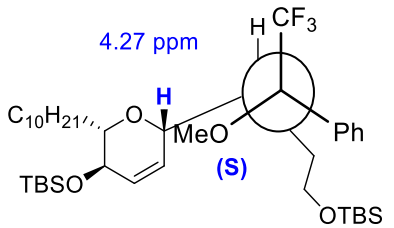

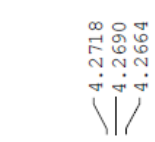

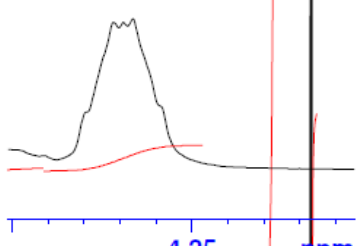

움

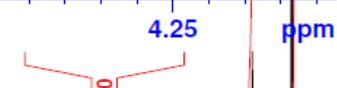

d.

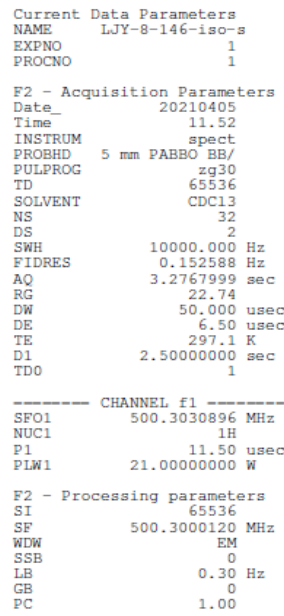

pm 


\section{Comparison of NMR data with reference}

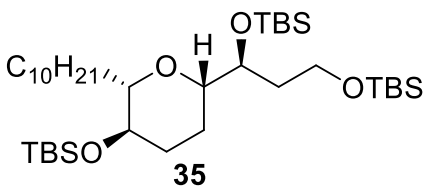

ref. $[\alpha]_{D}^{23}=-39.6\left(c=0.98, \mathrm{CHCl}_{3}\right)$ synthesis $[\alpha]_{D}^{21}=-38.6\left(\mathrm{c}=0.98, \mathrm{CHCl}_{3}\right)$

\begin{tabular}{|c|c|c|}
\hline & $\begin{array}{l}\text { Reference }^{7} \\
(300 \mathrm{MHz})\end{array}$ & $\begin{array}{c}\text { Synthesis 35 } \\
(500 \mathrm{MHz})\end{array}$ \\
\hline \multirow[t]{8}{*}{${ }^{1} \mathrm{H}$} & $3.78-3.74(\mathrm{~m}, 1 \mathrm{H})$ & $3.79-3.75(\mathrm{~m}, 1 \mathrm{H})$ \\
\hline & $3.69-3.61(\mathrm{~m}, 2 \mathrm{H})$ & $3.70-3.61(\mathrm{~m}, 2 \mathrm{H})$ \\
\hline & $3.24-3.19(\mathrm{~m}, 2 \mathrm{H})$ & $3.23-3.19(\mathrm{~m}, 2 \mathrm{H})$ \\
\hline & $3.02-2.93(\mathrm{~m}, 1 \mathrm{H})$ & $3.01-3.97(\mathrm{~m}, 1 \mathrm{H})$ \\
\hline & $2.04-1.93(\mathrm{~m}, 1 \mathrm{H})$ & $2.02-1.93(\mathrm{~m}, 1 \mathrm{H})$ \\
\hline & $1.84-1.39(\mathrm{~m}, 7 \mathrm{H}), 1.39-1.20(\mathrm{~m}, 16 \mathrm{H})$ & $\begin{array}{c}1.80-1.63(\mathrm{~m}, 3 \mathrm{H}), \begin{array}{c}1.56-1.50(\mathrm{~m}, 1 \mathrm{H}), 1.43-1.24 \\
(\mathrm{~m}, 19 \mathrm{H})\end{array} \\
.\end{array}$ \\
\hline & $0.89-0.85(\mathrm{~m}, 30 \mathrm{H})$ & $0.87-0.86(\mathrm{~m}, 30 \mathrm{H})$ \\
\hline & $0.03-0.02(\mathrm{~m}, 18 \mathrm{H})$ & $0.03-0.02(\mathrm{~m}, 18 \mathrm{H})$ \\
\hline \multirow[t]{22}{*}{${ }^{13} \mathrm{C}$} & 82.3 & 82.3 \\
\hline & 79.8 & 79.8 \\
\hline & 71.1 & 71.1 \\
\hline & 70.9 & 70.9 \\
\hline & 60.1 & 60.1 \\
\hline & 35.5 & $35.5,33.5$ \\
\hline & 32.0 & 32.0 \\
\hline & 31.9 & 31.9 \\
\hline & 29.8 & 29.8 \\
\hline & 29.7 & $29.68,29.66$ \\
\hline & 29.4 & 29.3 \\
\hline & 26. & $25.99,25.96$ \\
\hline & 25.9 & 25.92 \\
\hline & 25.7 & 25.8 \\
\hline & 25.2 & 25.1 \\
\hline & 25.1 & 25.0 \\
\hline & 22.7 & 22.7 \\
\hline & 18.2 & $18.3,18.2,18.0$ \\
\hline & 14.1 & 14.1 \\
\hline & -4.3 & -4.0 \\
\hline & -4.4 & -4.4 \\
\hline & -4.8 & $-4.74,-4.76,-5.22,-5.29$ \\
\hline
\end{tabular}




\section{Reference}

(1) Wittman, M. D.; Halcomb, R. L.; Danishefsky, S. J.; Golik, J.; Vyas, D. A route to glycals in the allal and gulal series: synthesis of the thiosugar of esperamicin A1. J. Org. Chem. 1990, 55, 1979-1981.

(2) Mizia, J. C.; Bennett, C. S., Reagent Controlled Direct Dehydrative Glycosylation with 2-Deoxy Sugars: Construction of the Saquayamycin Z Pentasaccharide. Org. Lett. 2019, 21, 5922-5927.

(3) Takeshi, S.; Hanako, O.; Atsuhito, K.; Hiromichi, O. A Chemo-Enzymatic Synthesis of D-Allosamine Derivatives from Tri-O-acetyl-D-glucal. Bull. Chem. Soc. Jpn. 1997, 70, 2535-2540.

(4] Hansen, T. M.; Florence, G. J.; Lugo-Mas, P.; Chen, J.; Abrams, J. N.; Forsyth, C. J, Highly chemoselective oxidation of 1,5-diols to $\delta$-lactones with TEMPO/BAIB. Tetrahedron Lett. 2003, 44, 57-59.

(5) Dunkerton, L. V.; Euske, J. M.; Serino, A. J. Palladium(0)-assisted synthesis of C-glycopyranosyl compounds. Carbohydr. Res. 1987, 171, 89-107.

(6] Miyakoshi, T.; Togashi, H. Synthesis of 3-Alkylcatechols via Intramolecular Cyclization. Synthesis. 1990, 1990, 407-410.

(7) Hoppen, S.; Bäurle, S.; Koert, U. A Convergent Total Synthesis of (-)-Mucocin: An Acetogenin from Annonaceae. Chem. Eur. J. 2000, 6, 2382-2396. 


\section{9. ${ }^{1} \mathrm{H}$ and ${ }^{13} \mathrm{C}$ NMR of new compounds}
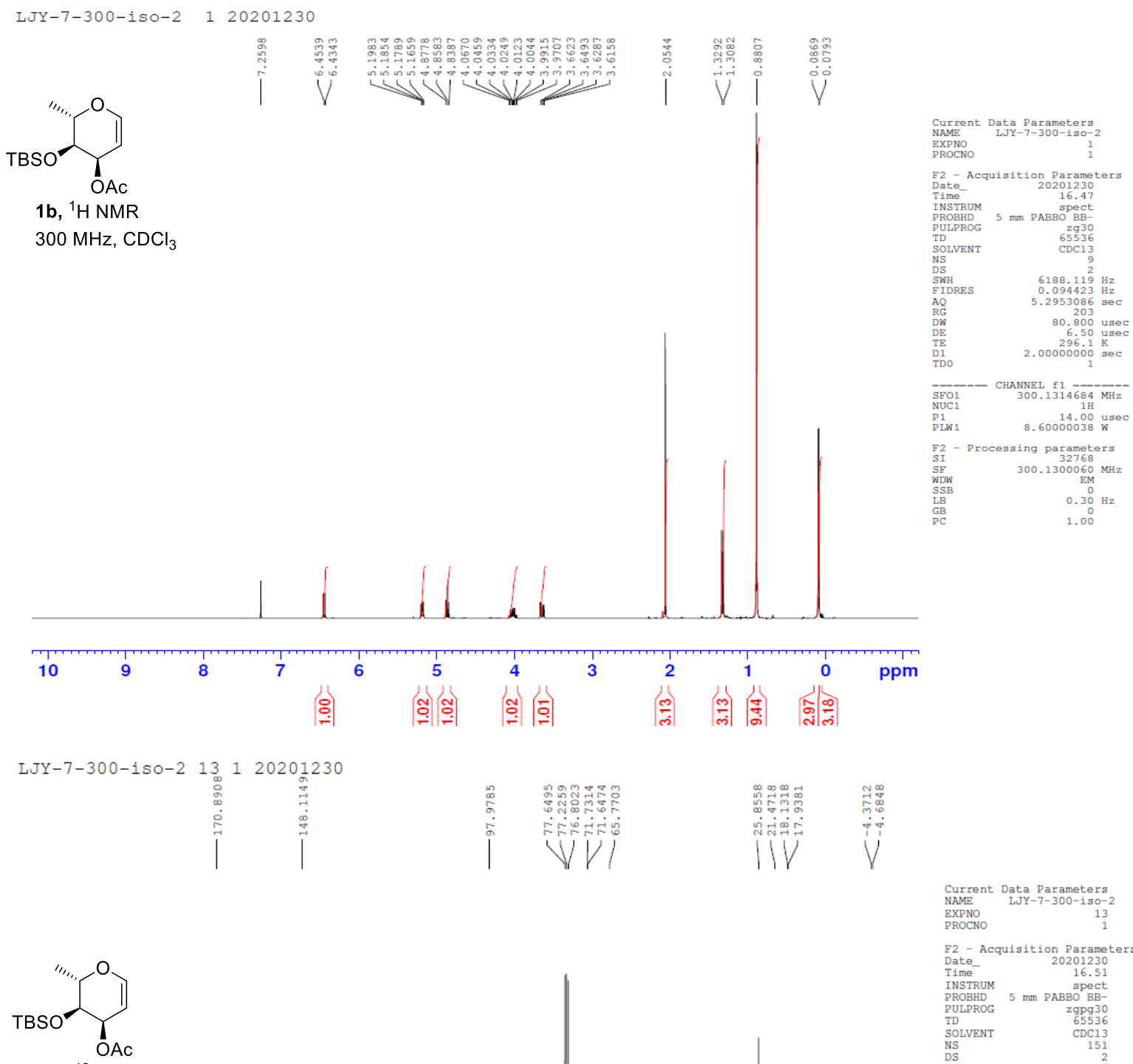

1b, ${ }^{13} \mathrm{C}$ NMR

$75 \mathrm{MHz} \mathrm{CDCl}_{3}$

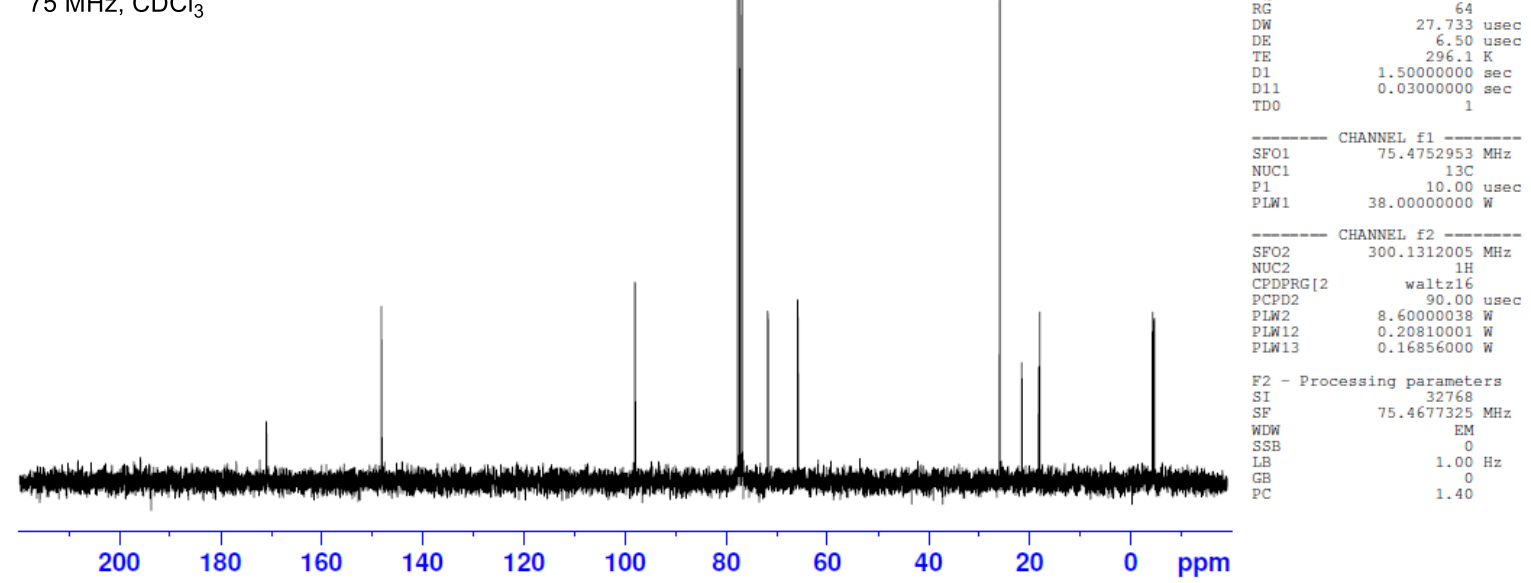



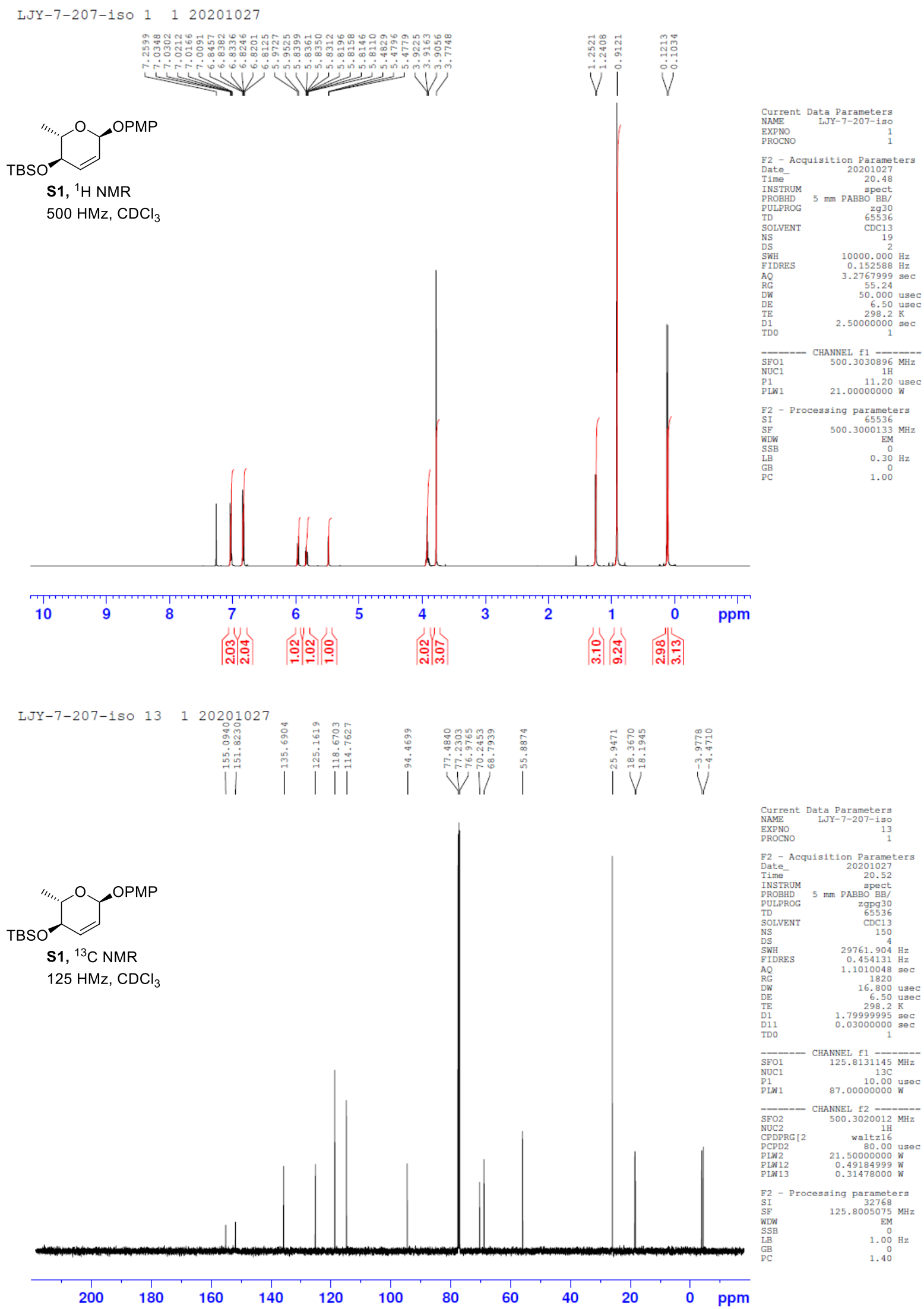


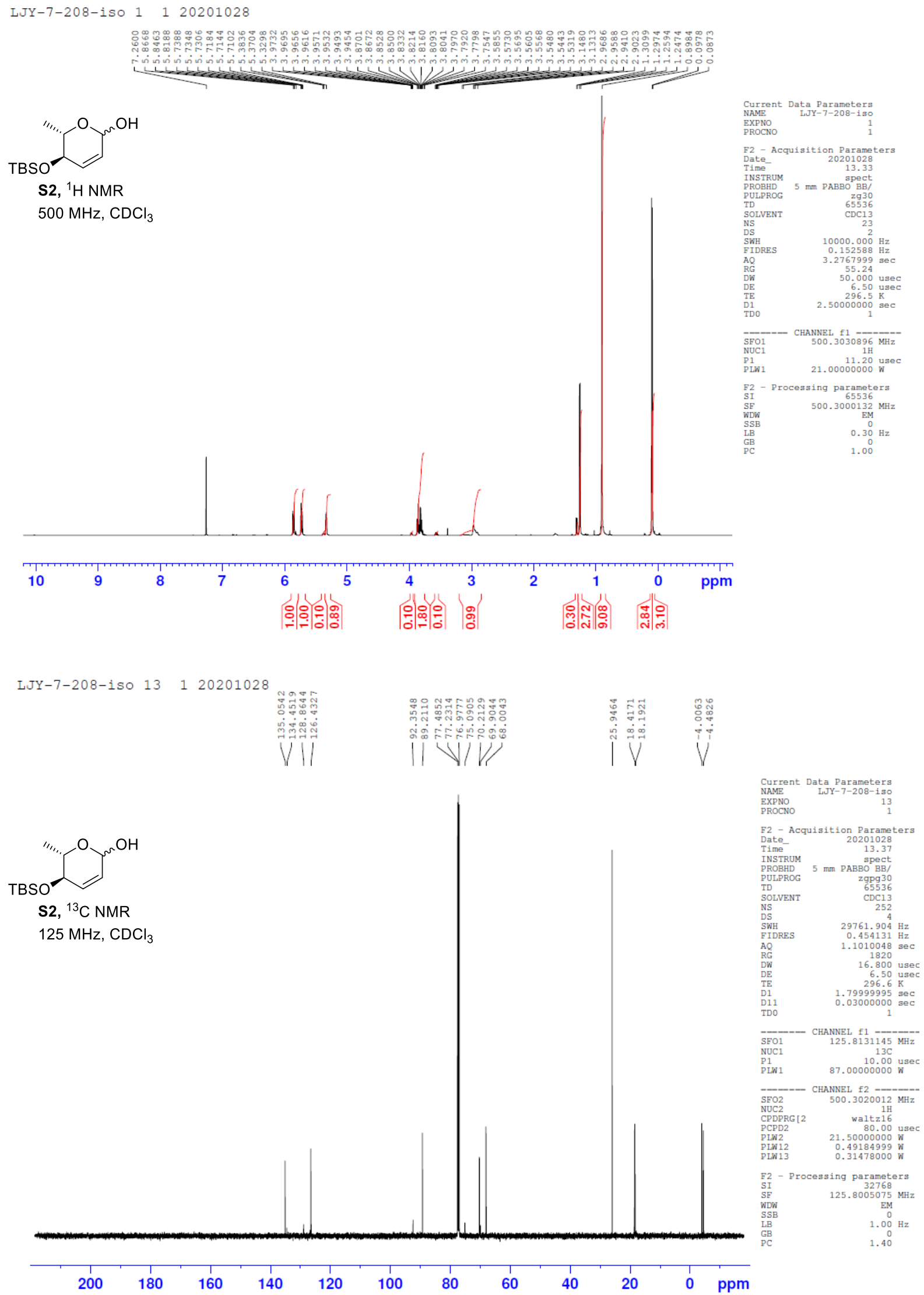



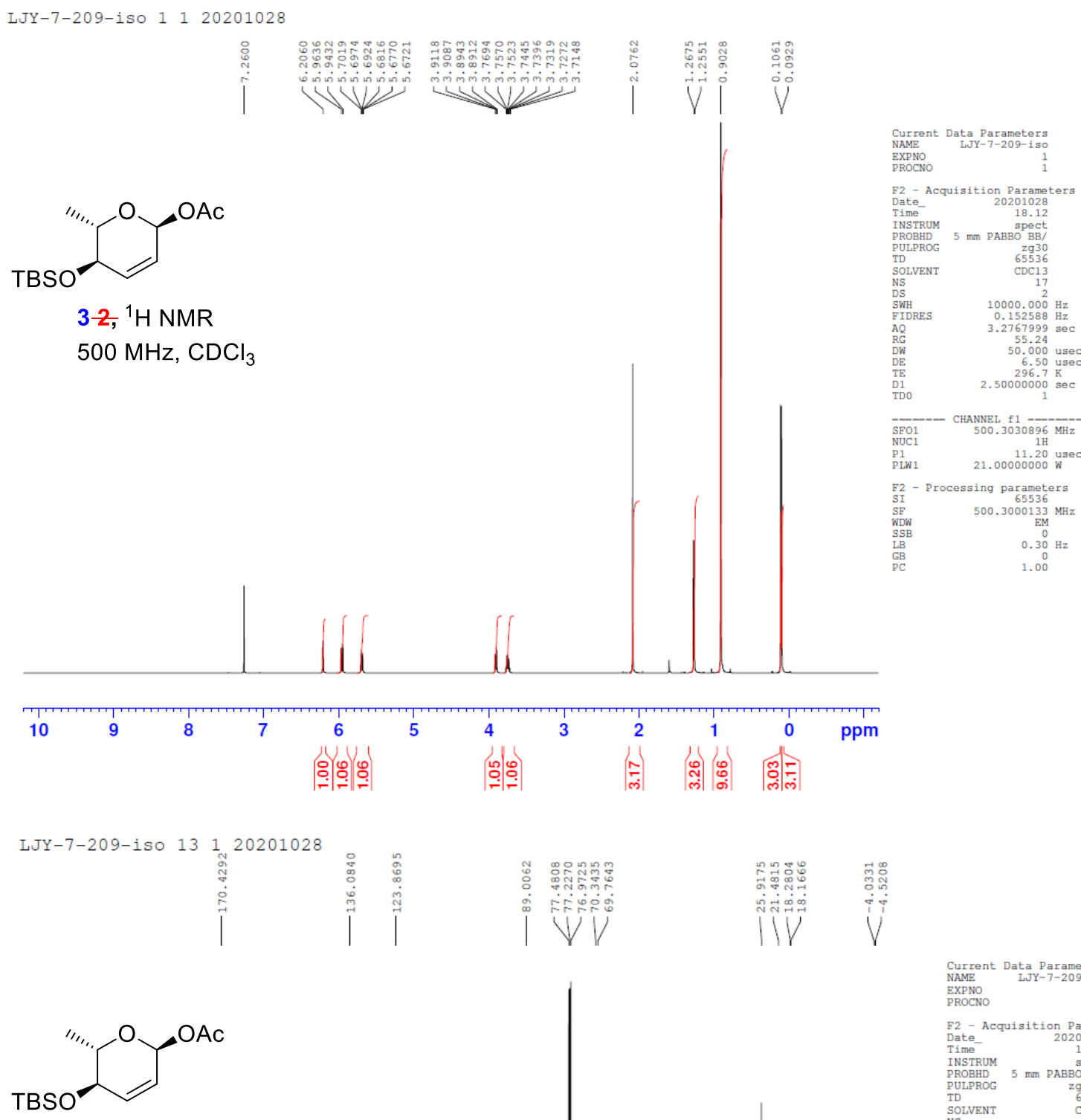

$32,{ }^{13} \mathrm{C} N M R$

$125 \mathrm{MHz}, \mathrm{CDCl}_{3}$
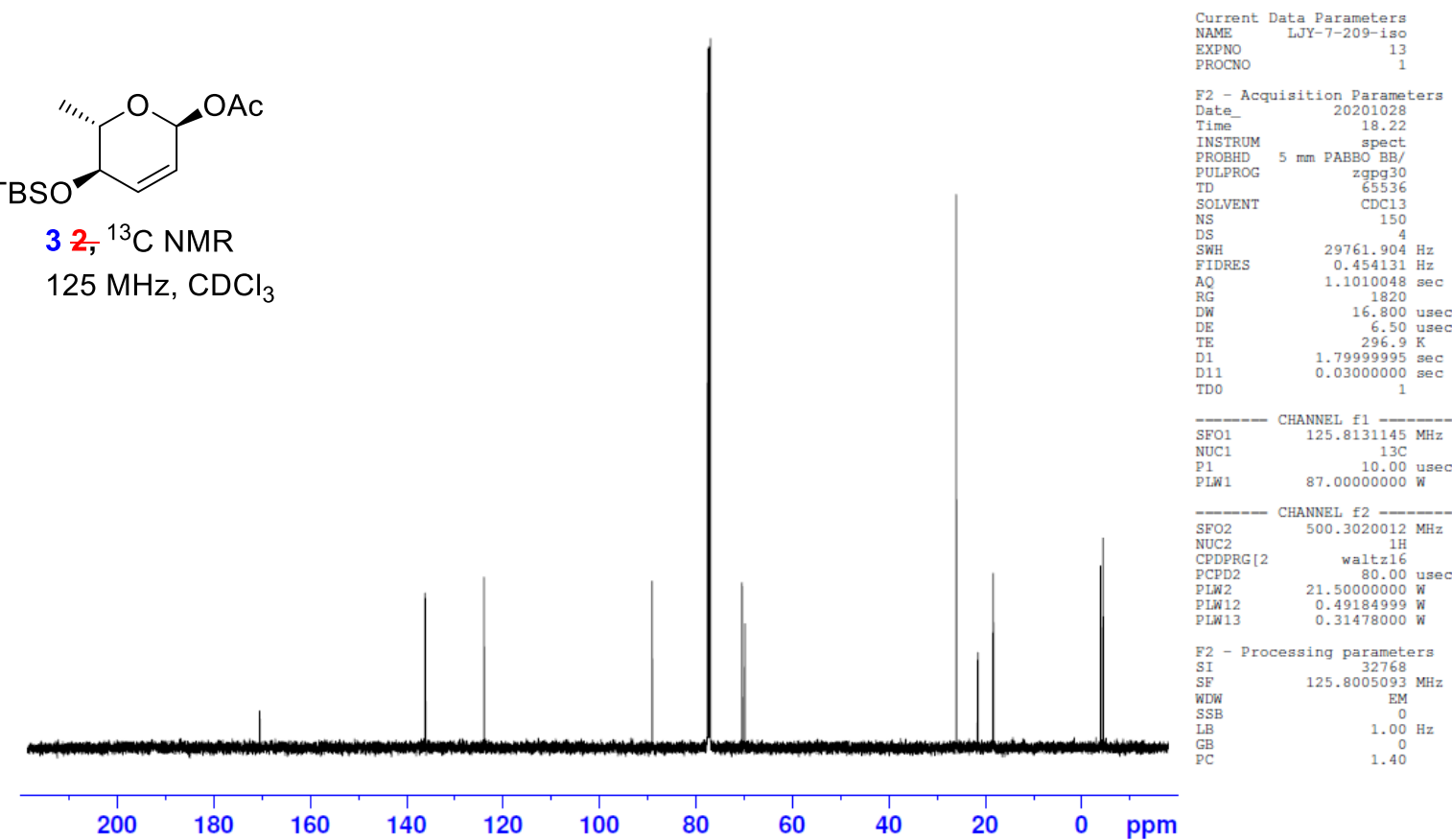

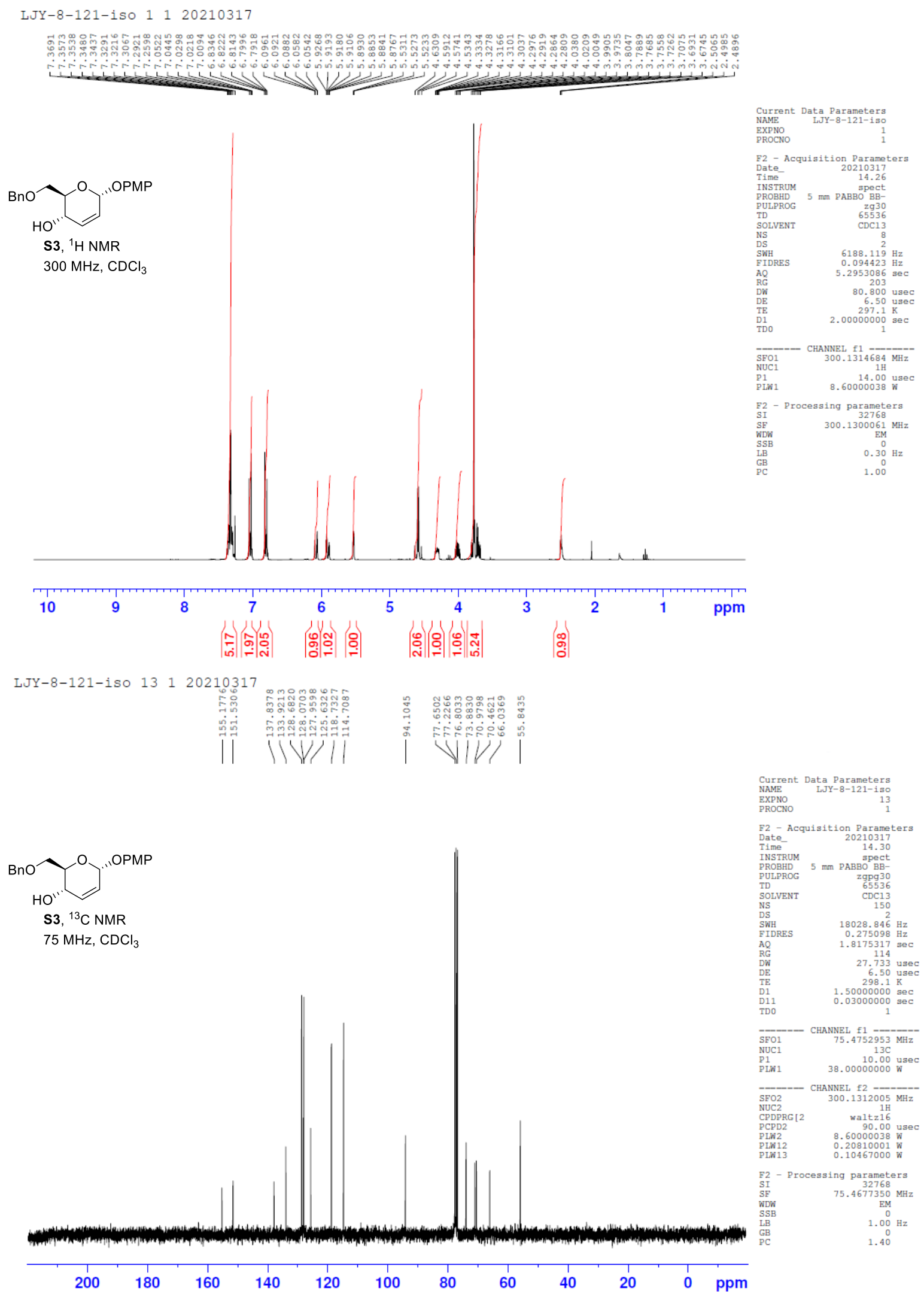


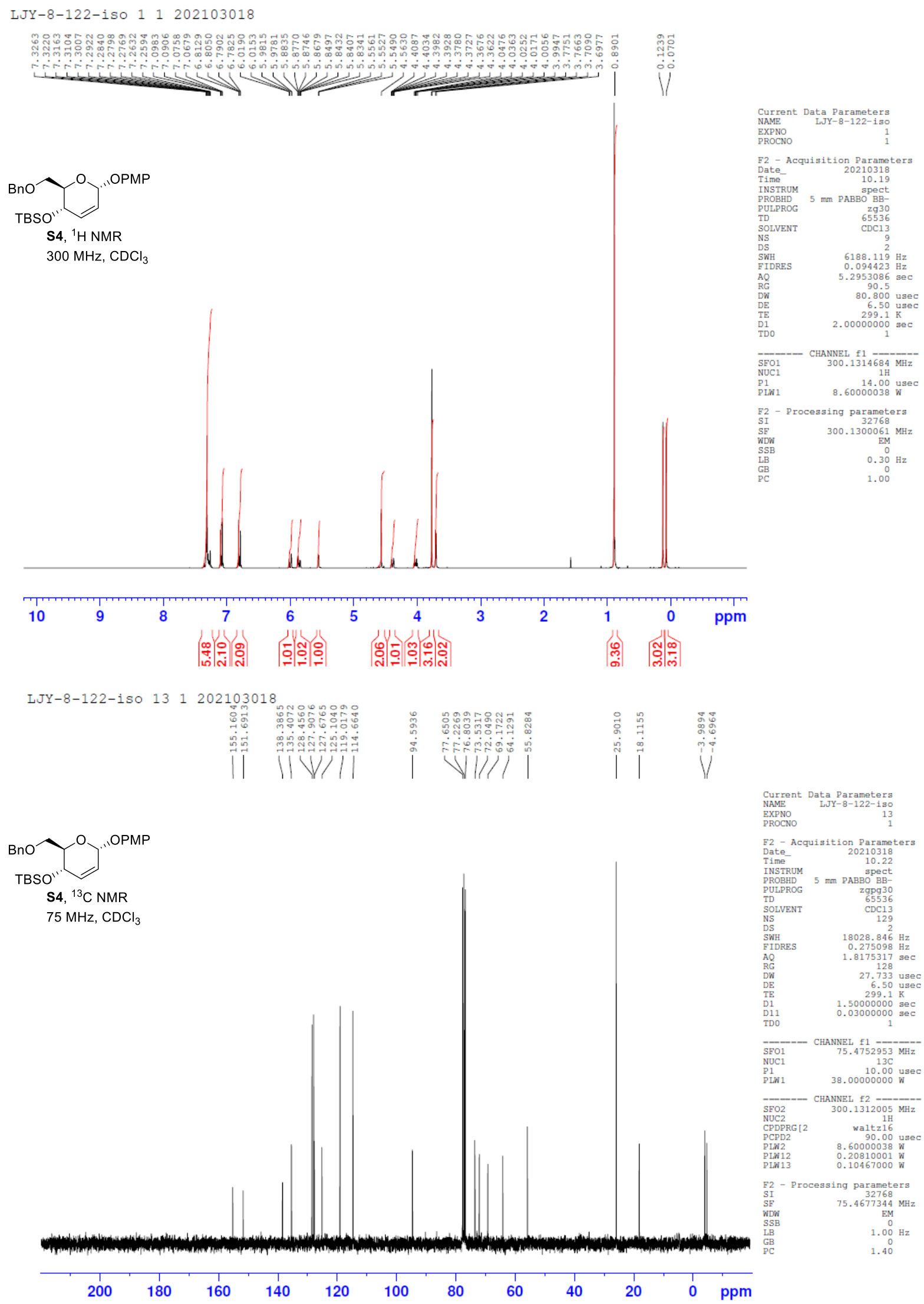



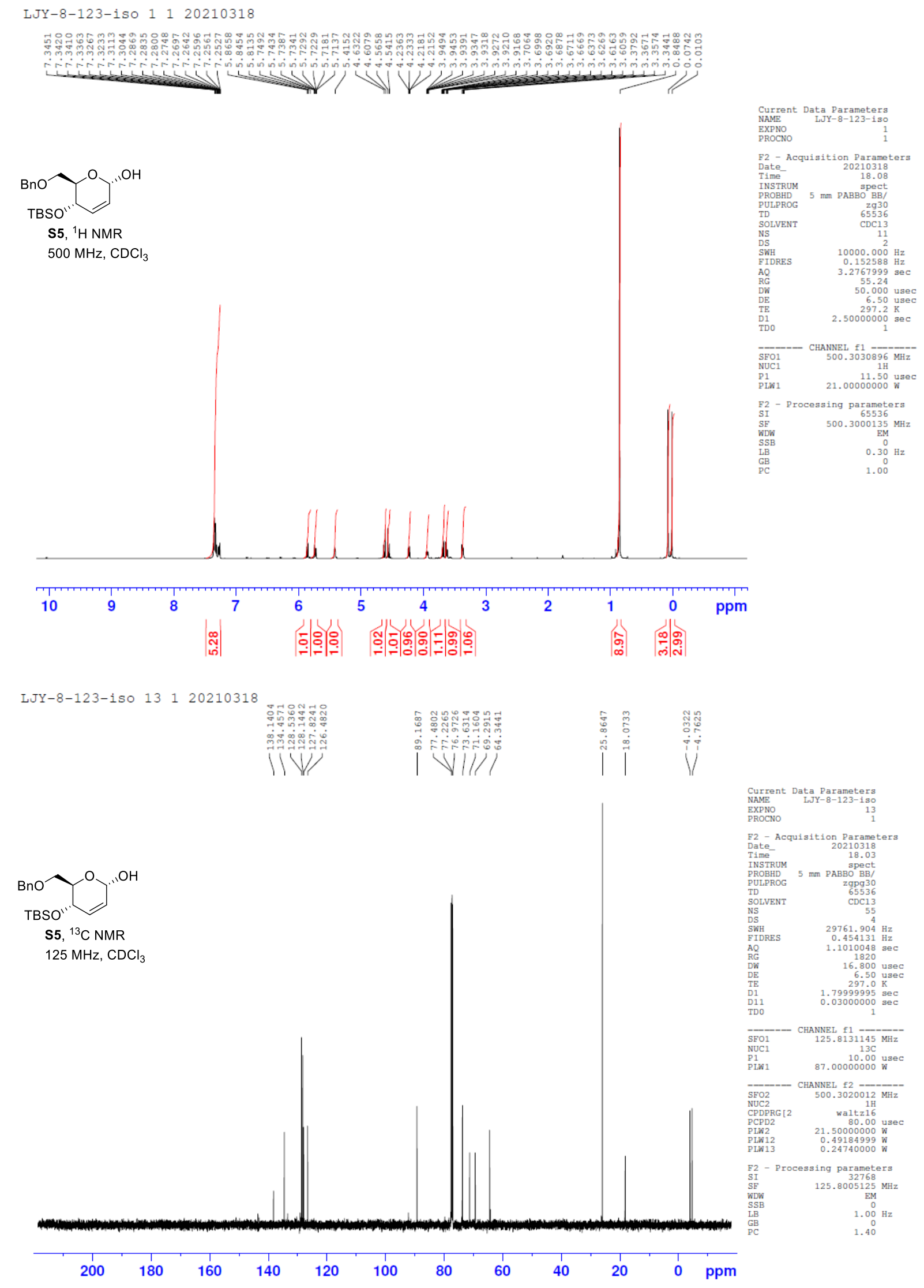

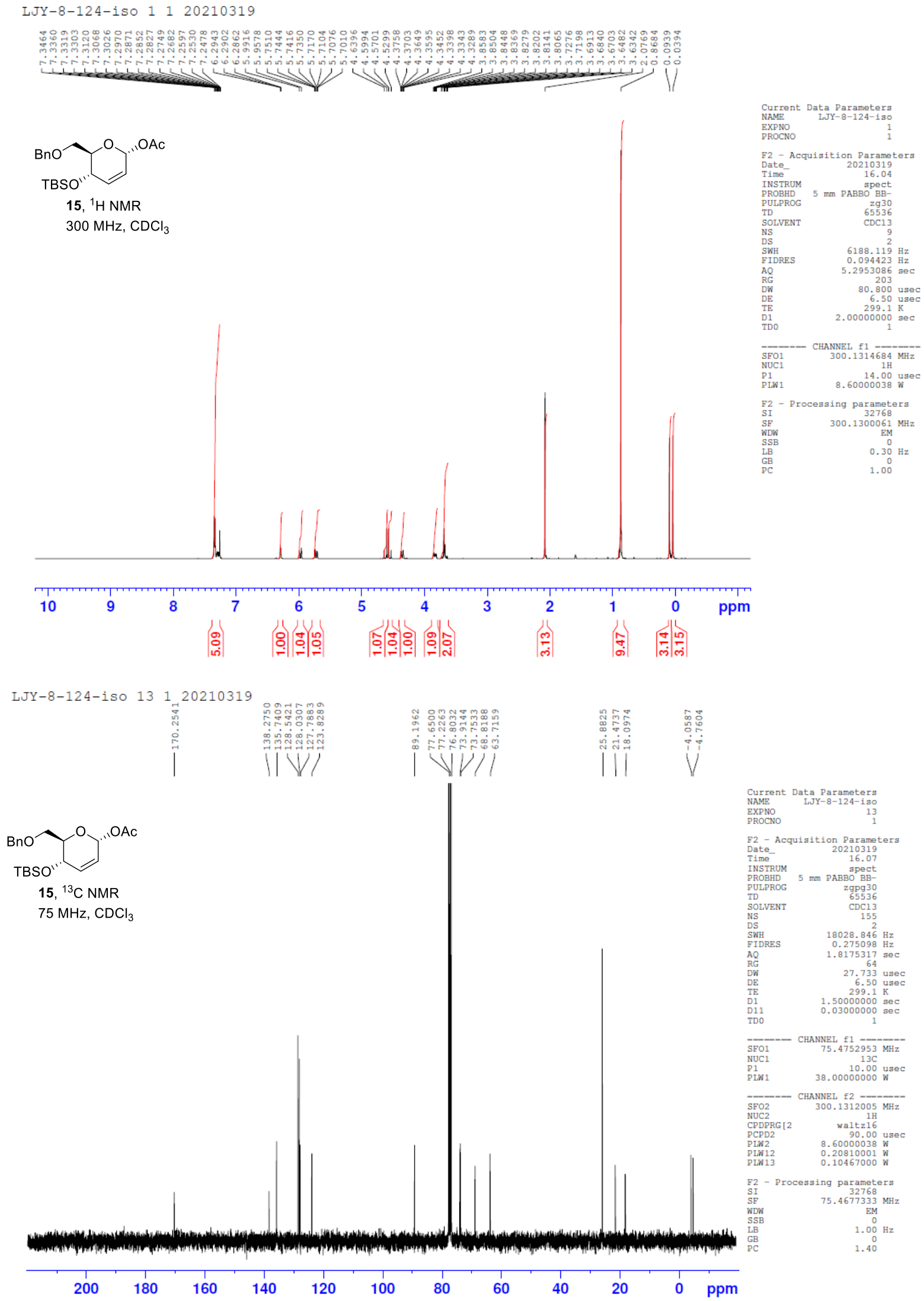

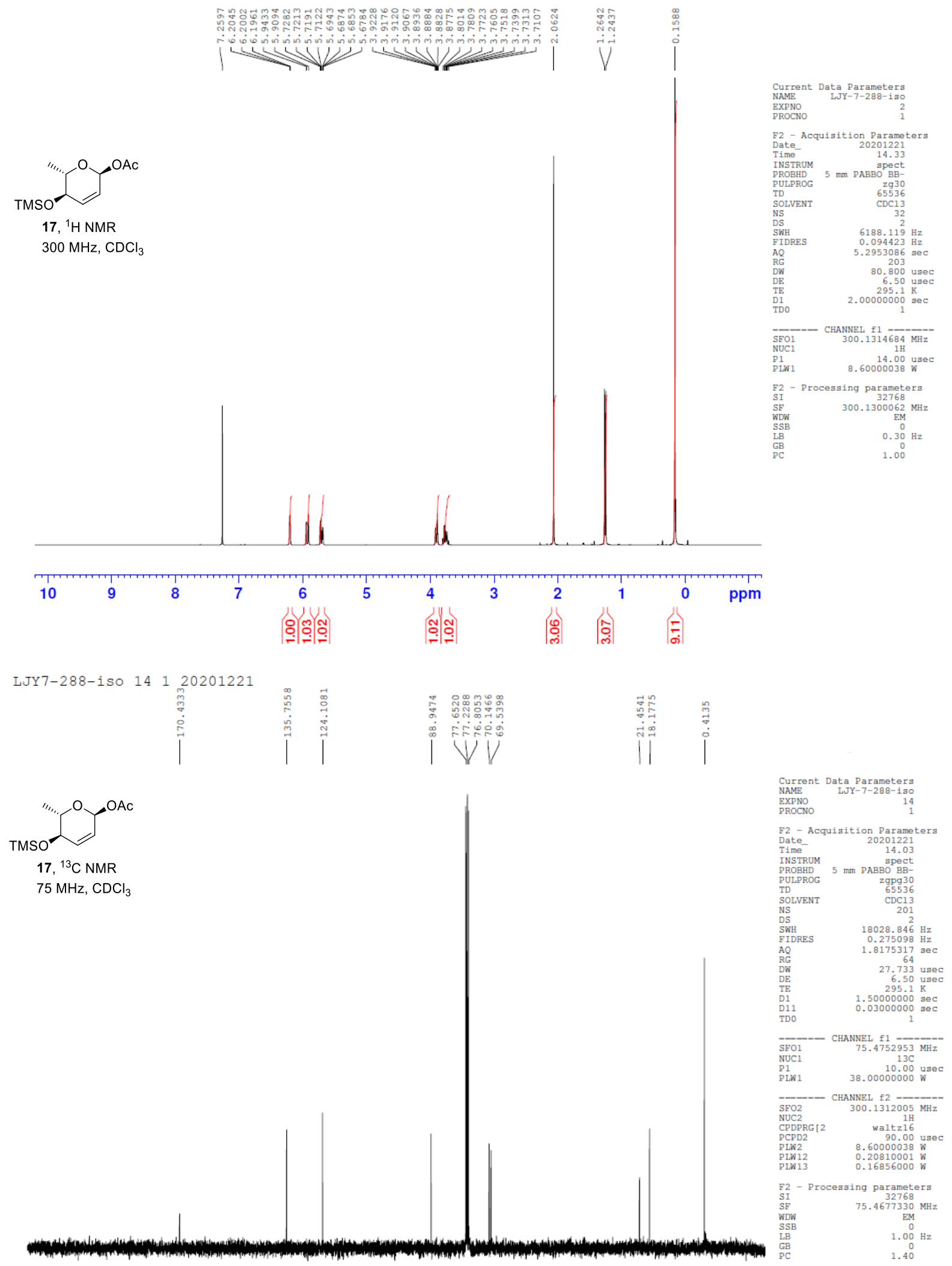


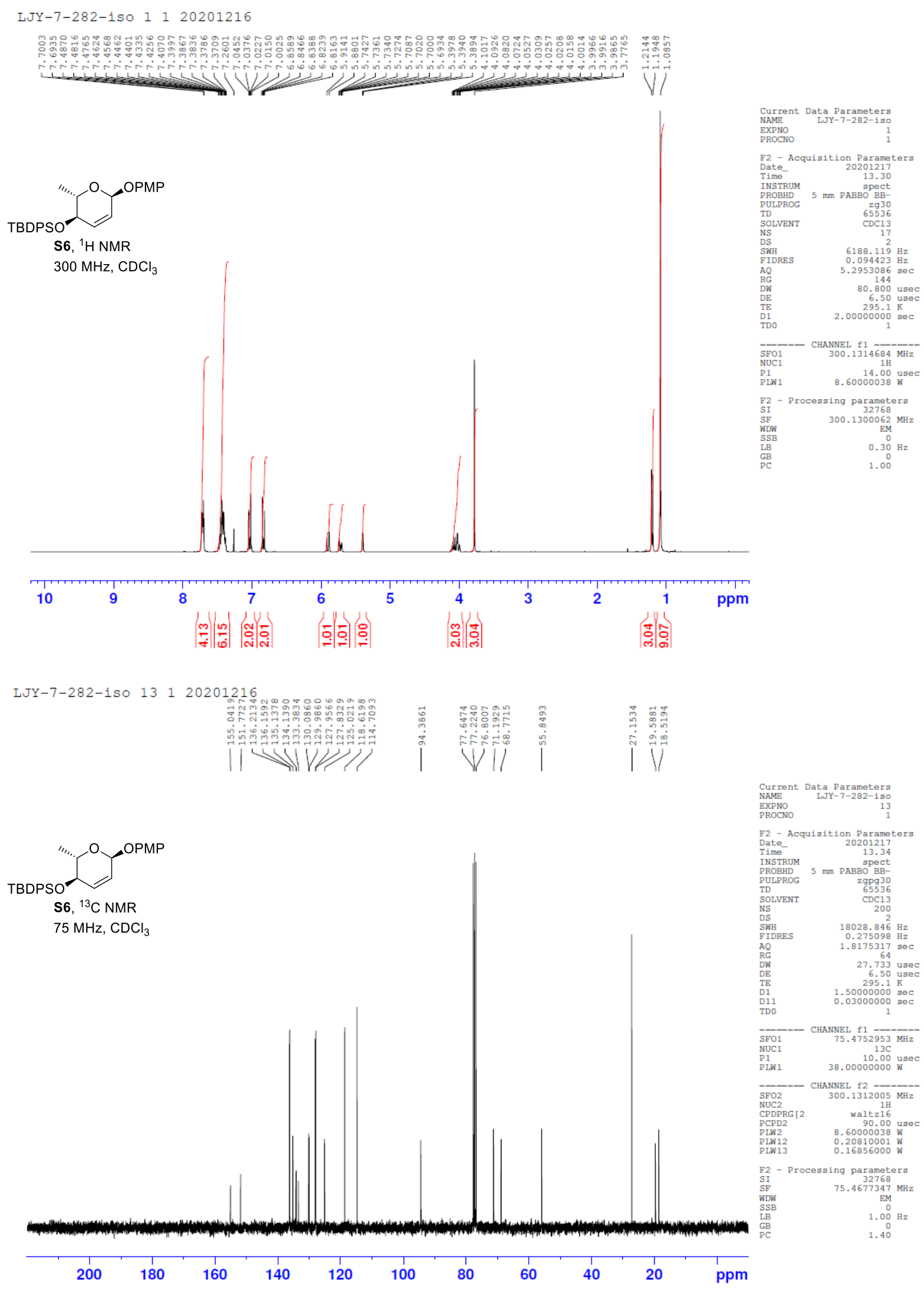



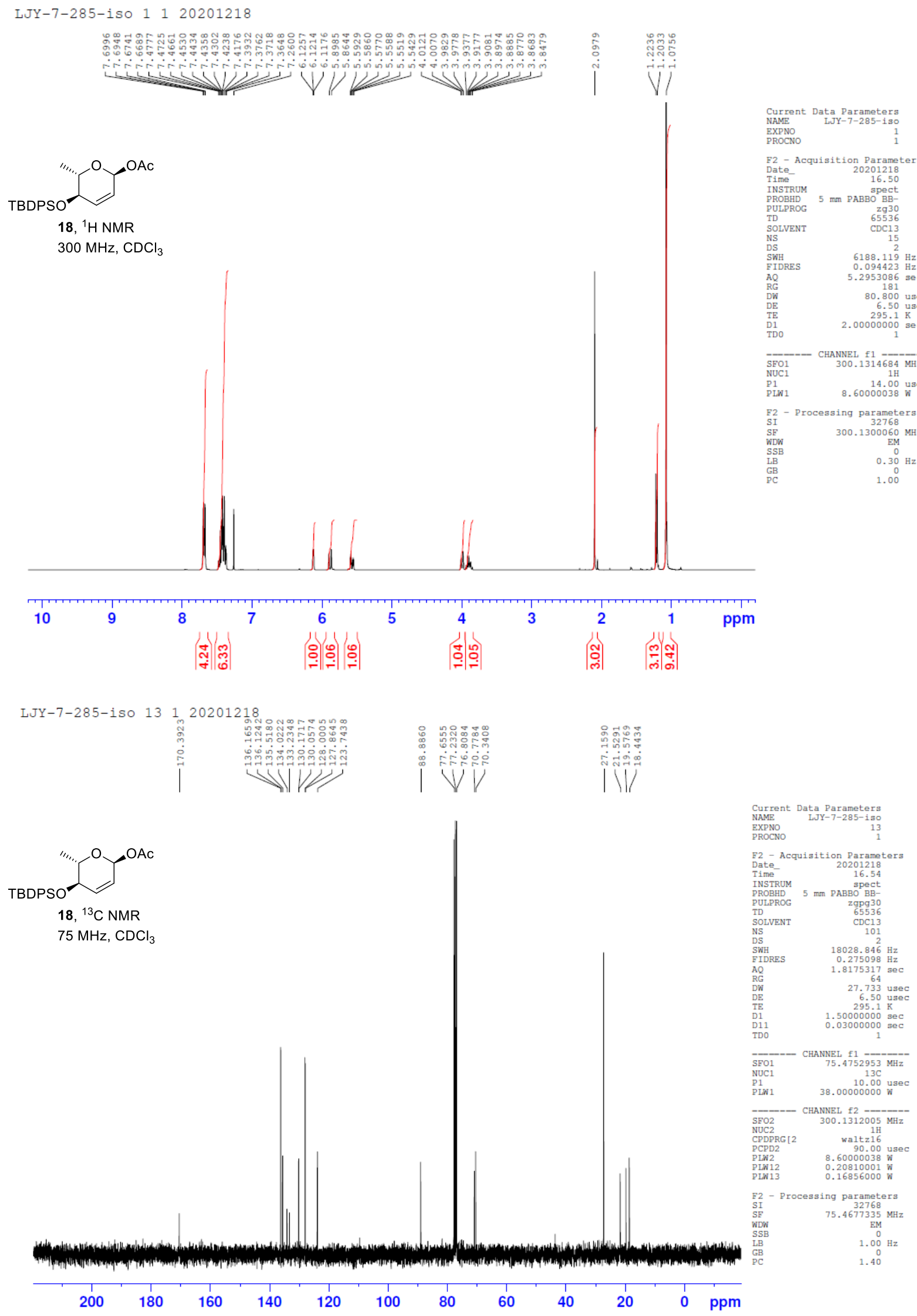


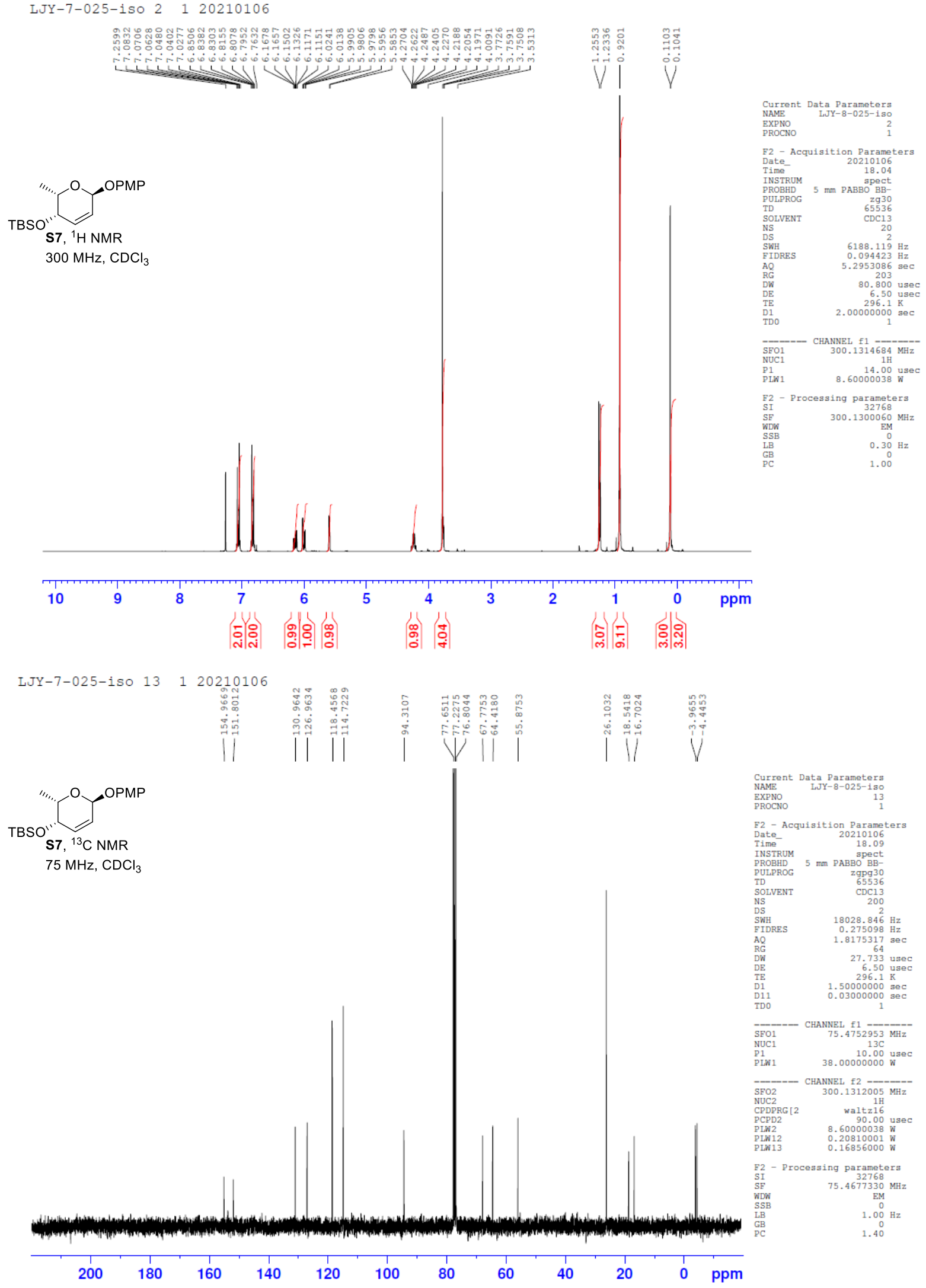




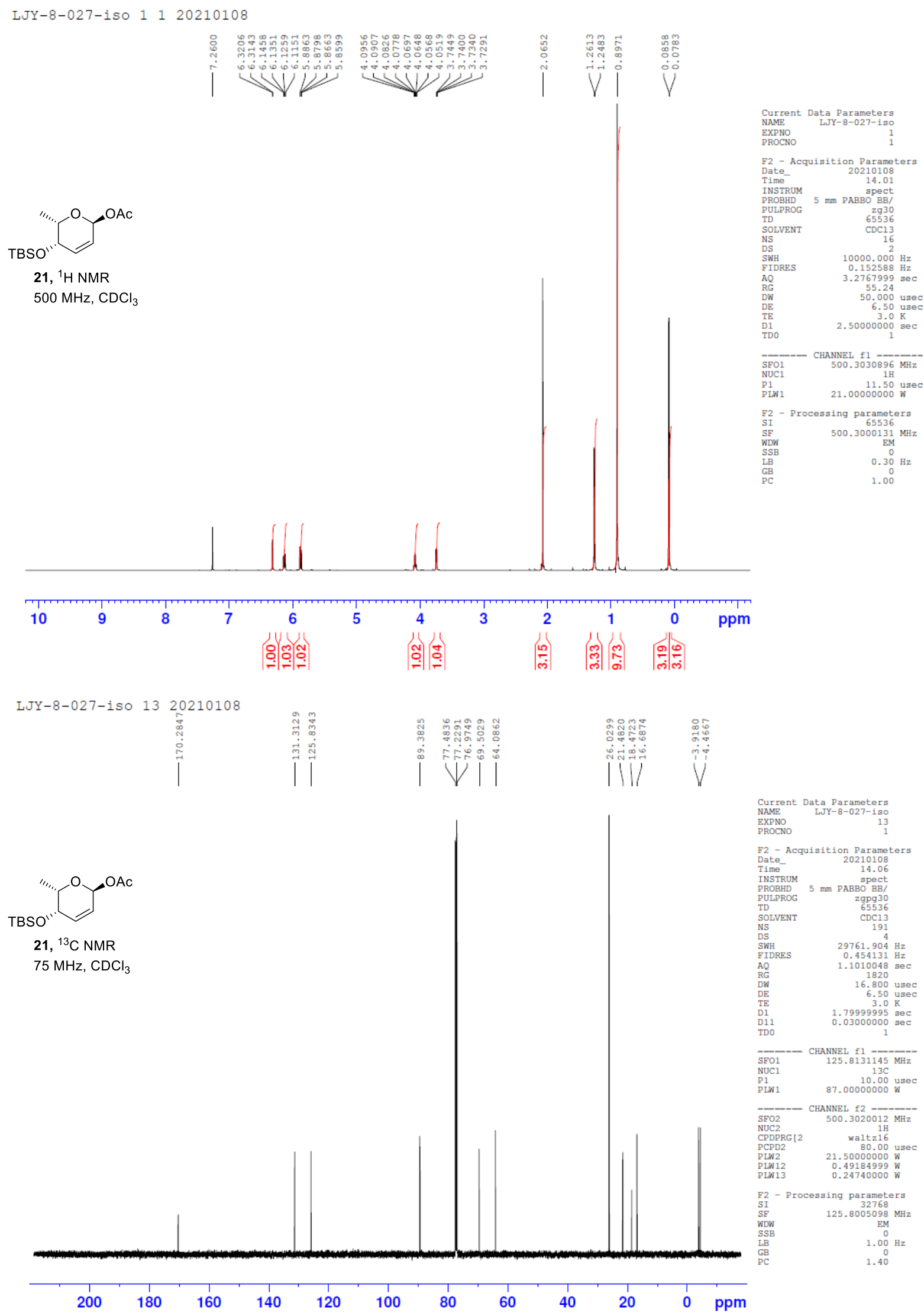




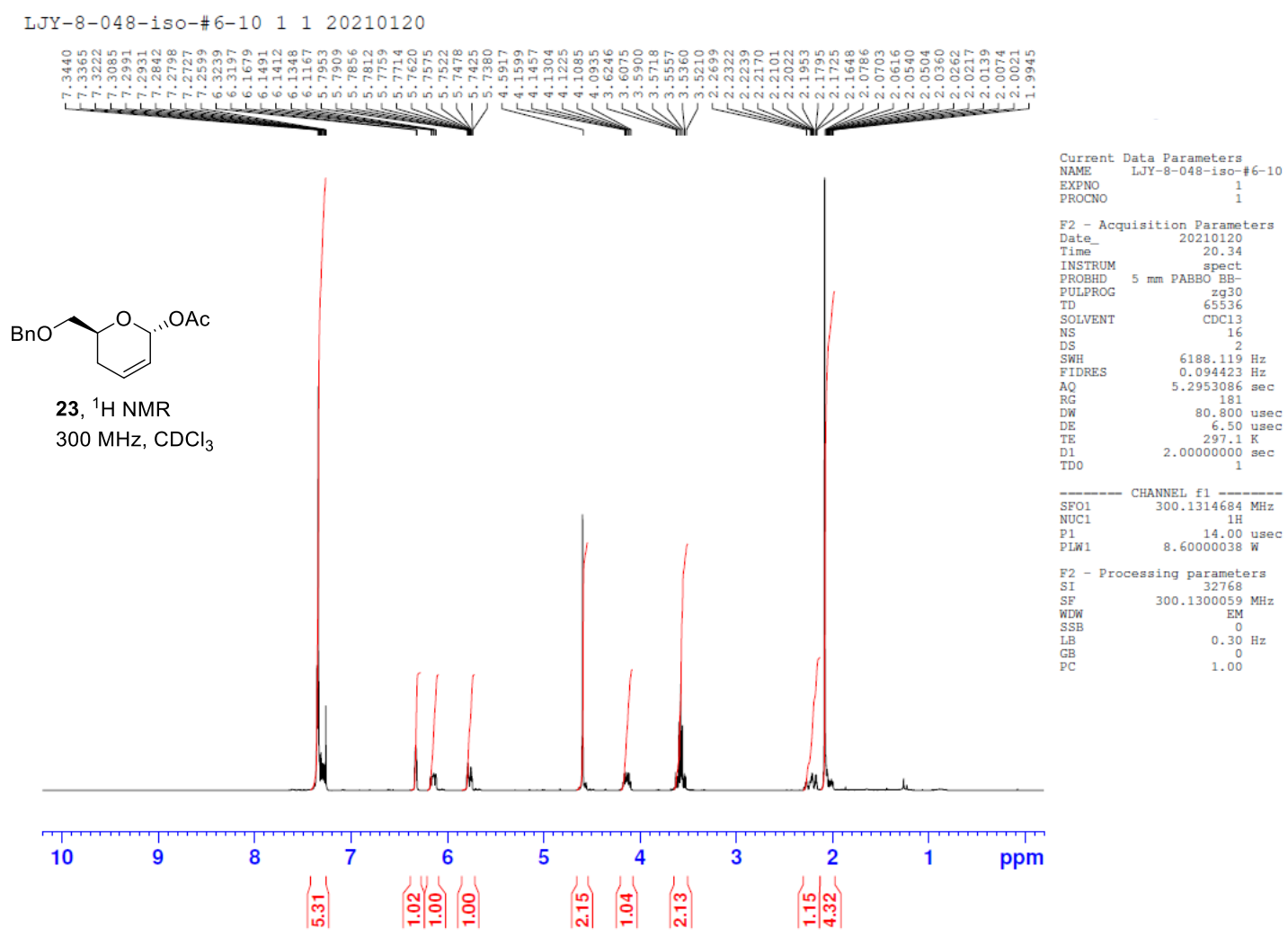

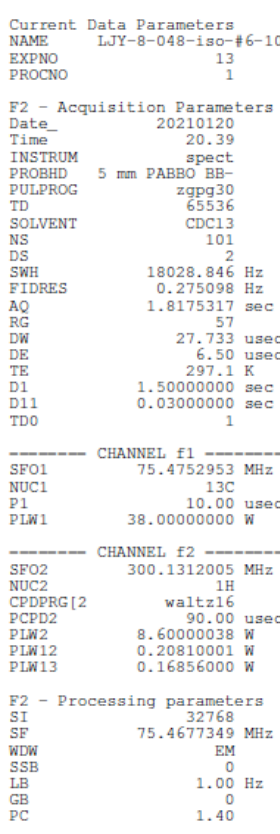

23, ${ }^{13} \mathrm{C}$ NMR

$75 \mathrm{MHz}, \mathrm{CDCl}_{3}$
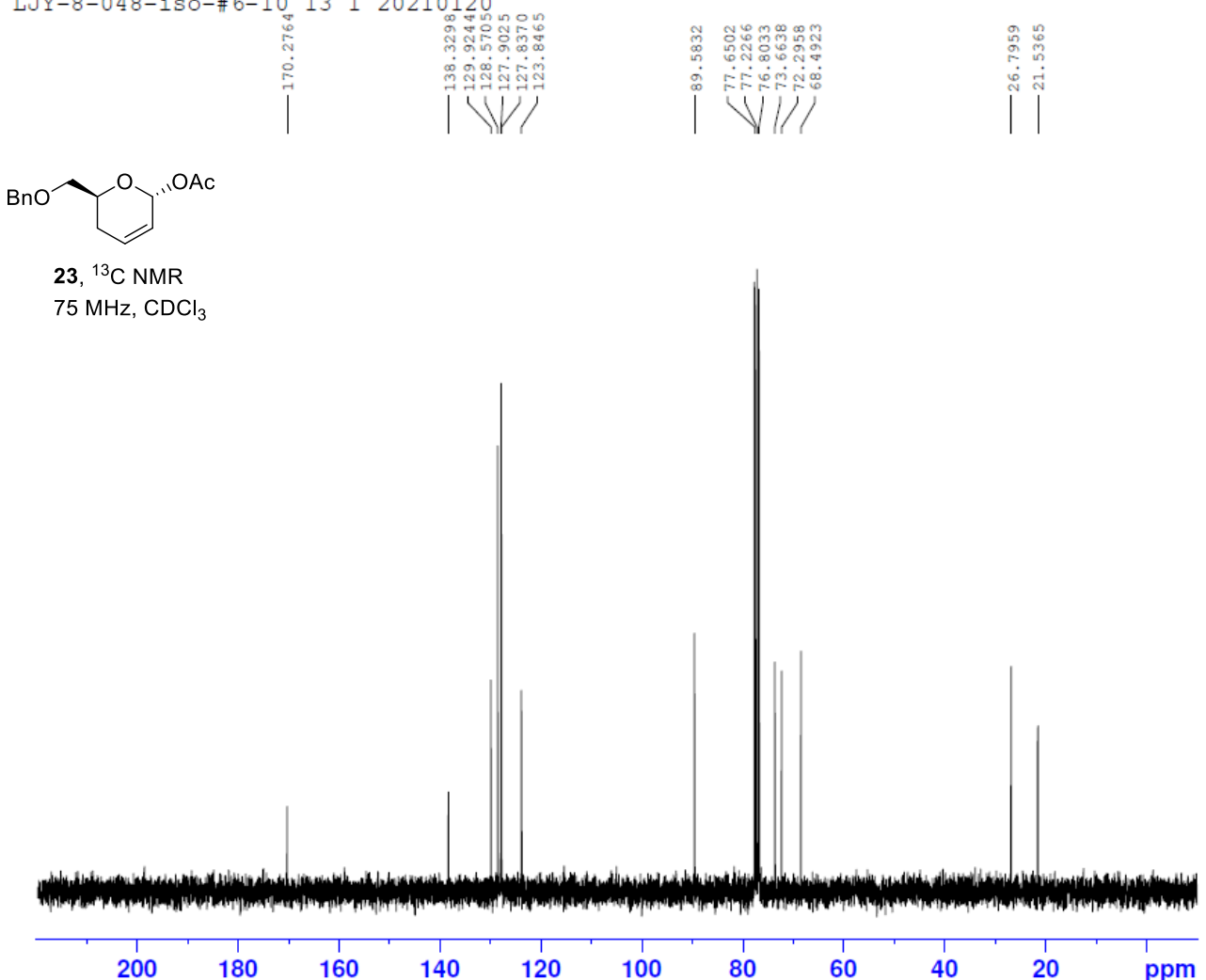


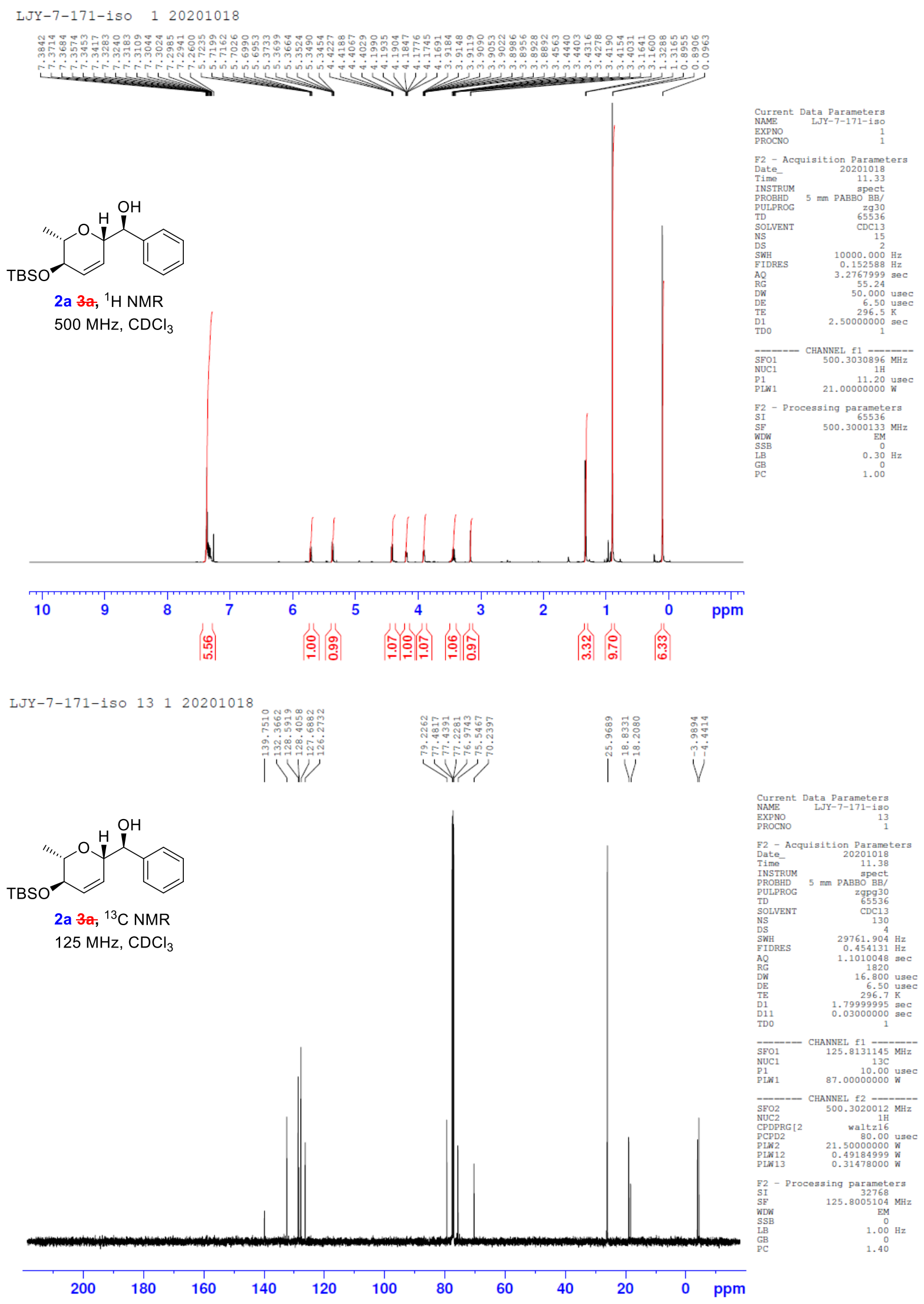



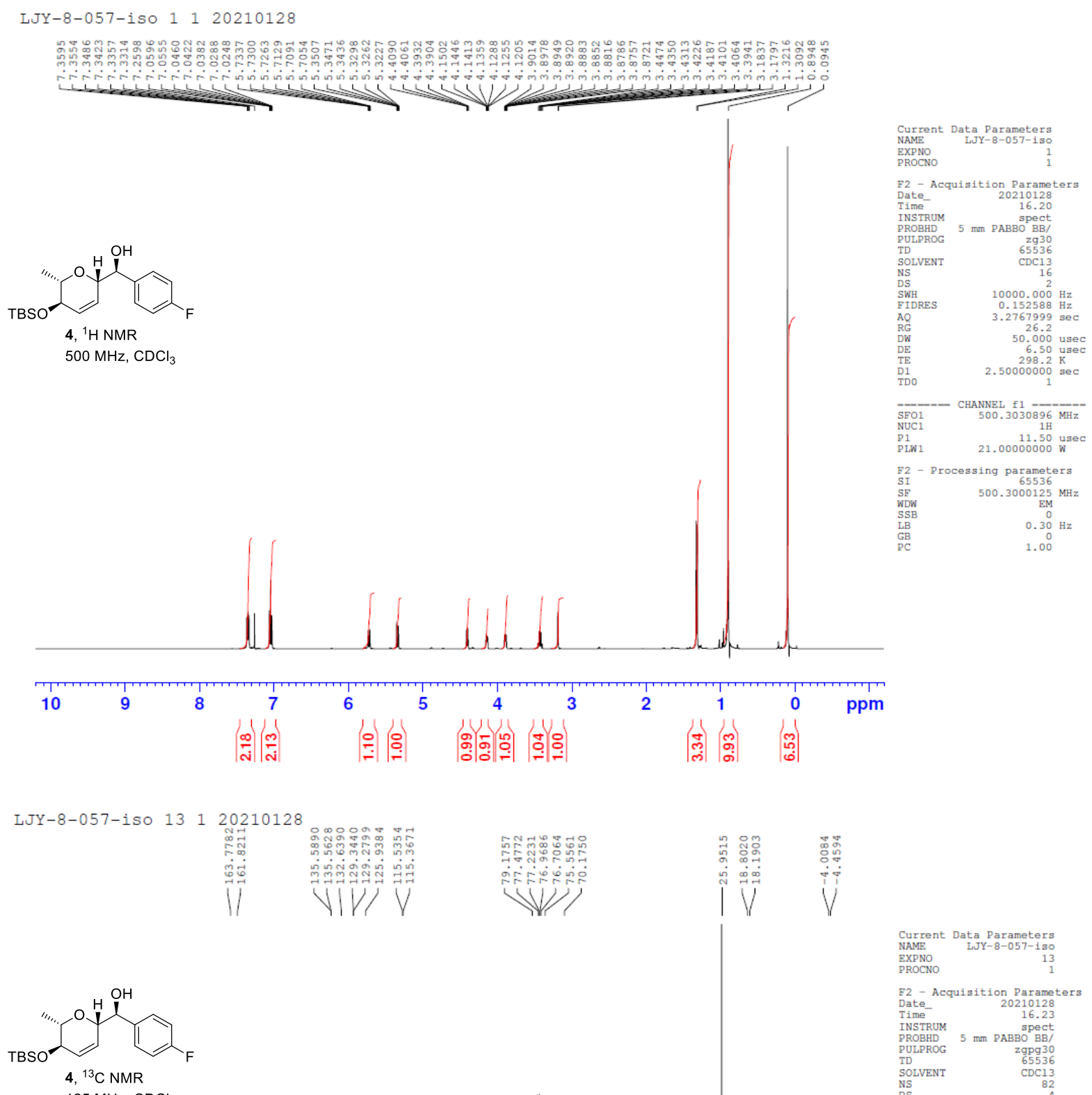

$125 \mathrm{MHz}, \mathrm{CDCl}_{3}$
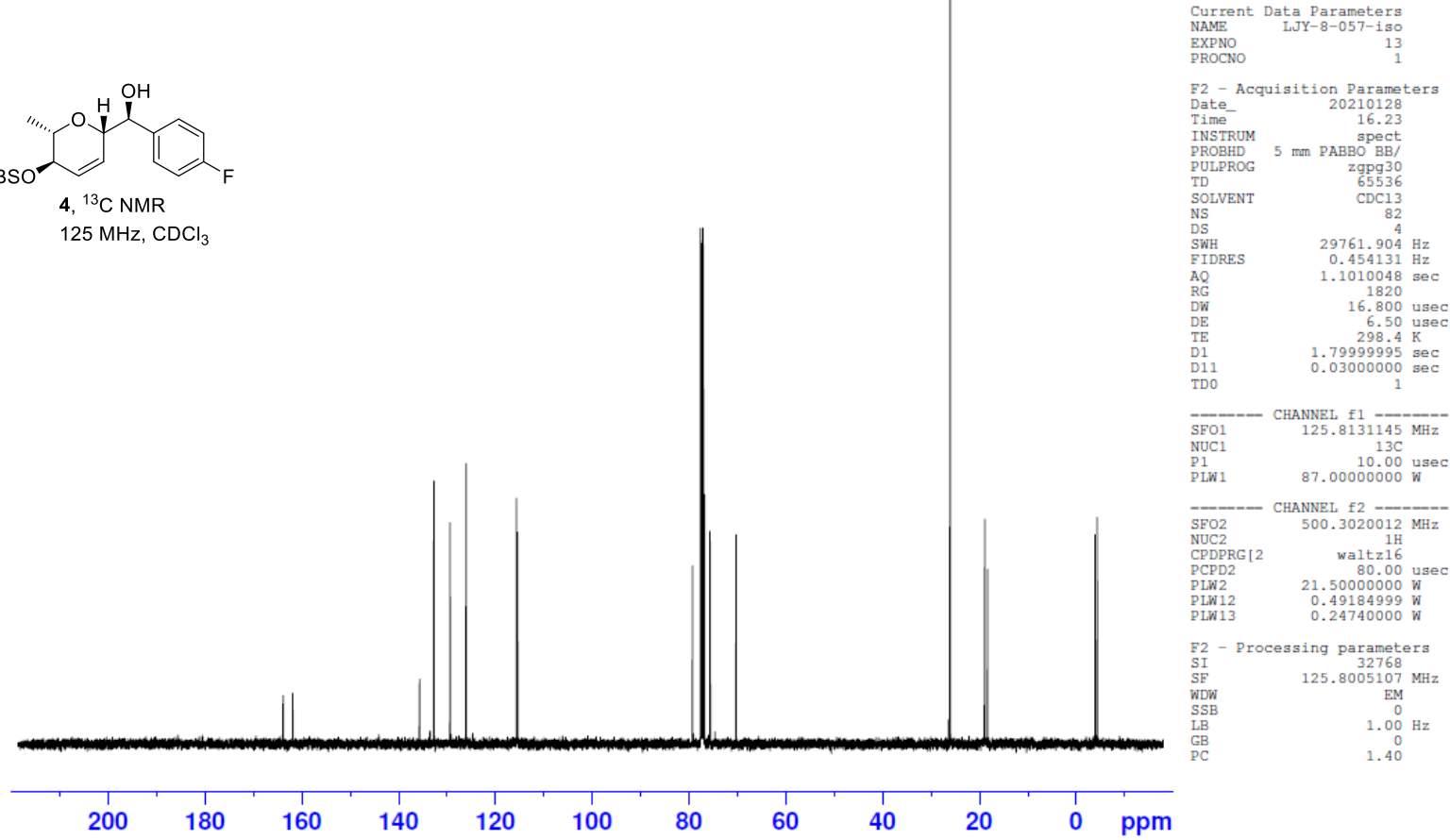

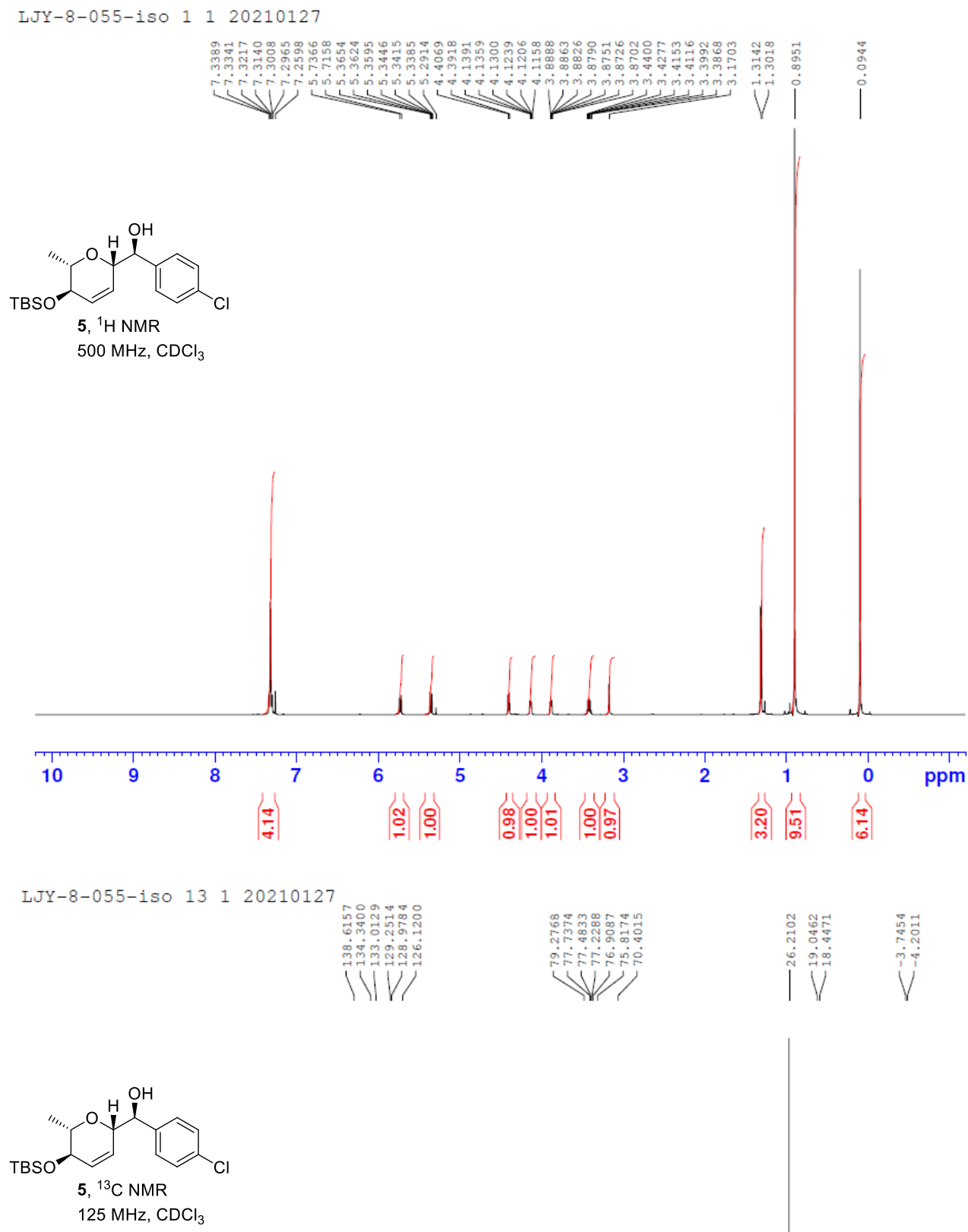

Current Data Parameters
NAME LUY-8-055-iso
EXPNO

EXPNO
PROCNO

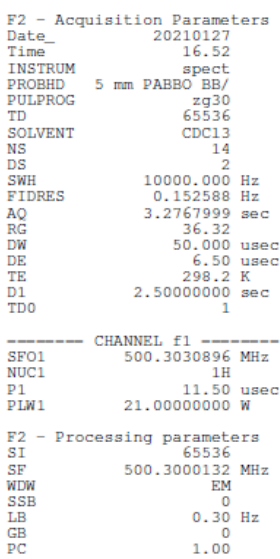

$125 \mathrm{MHz}, \mathrm{CDCl}_{3}$

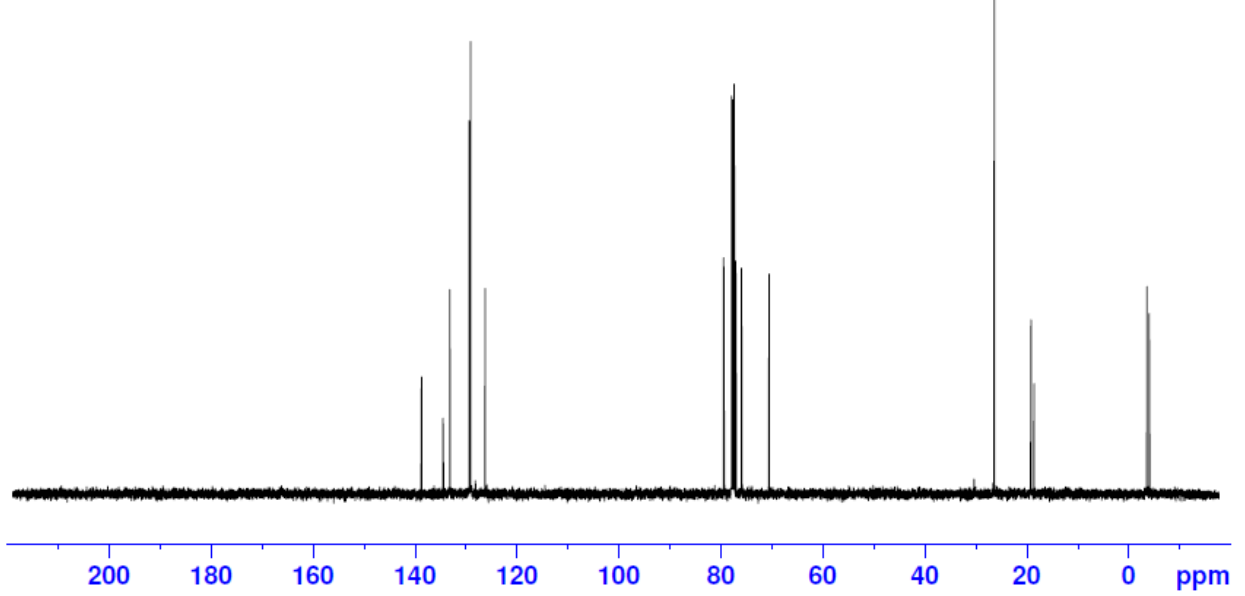

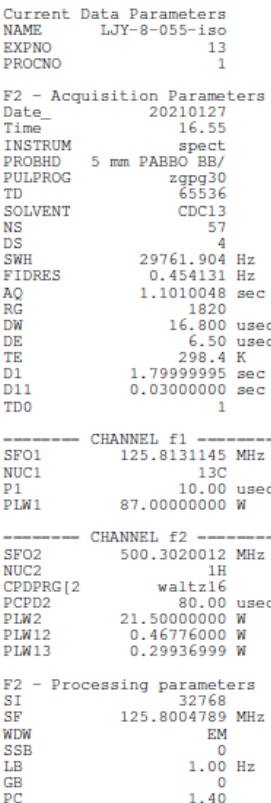



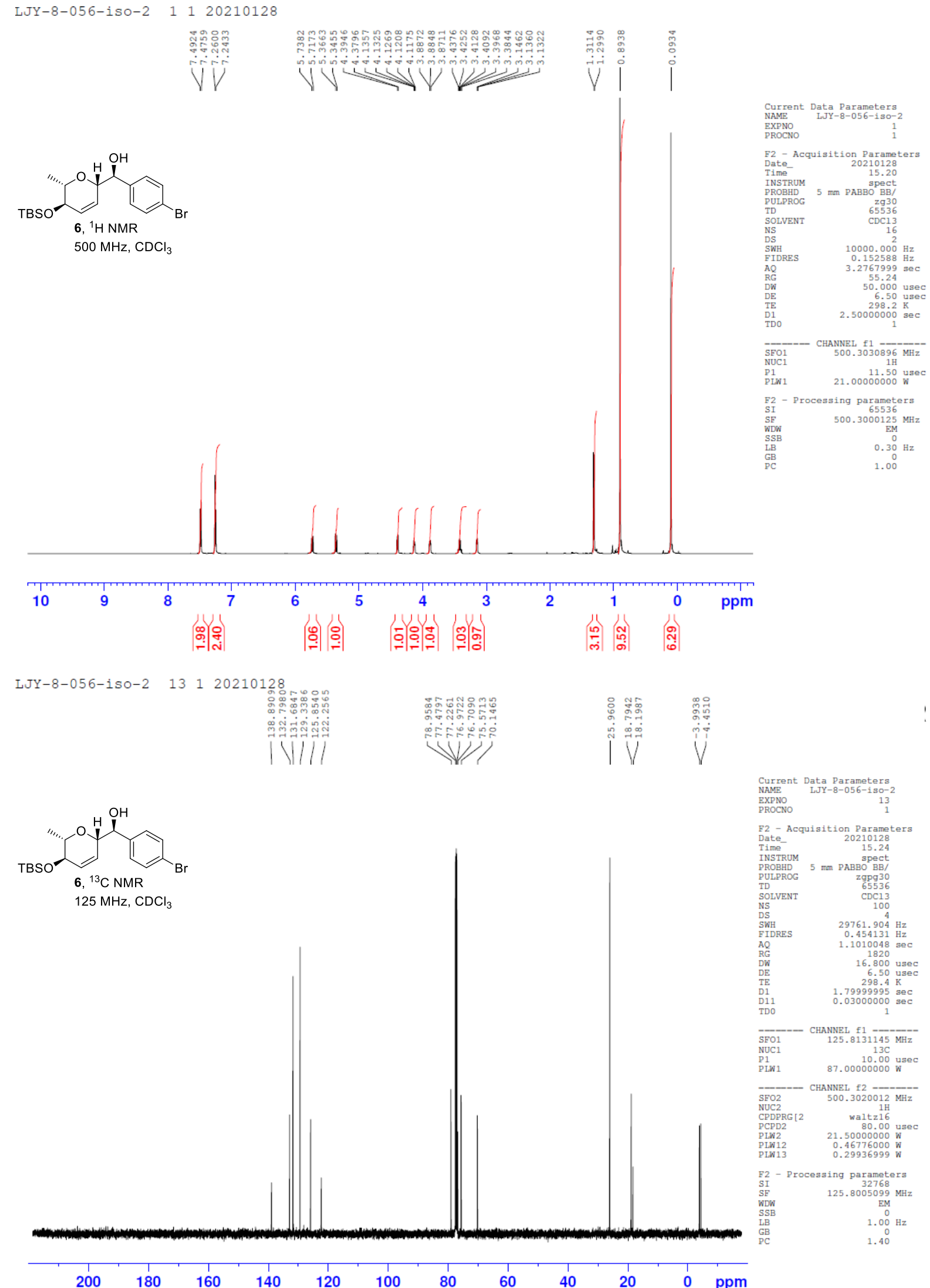


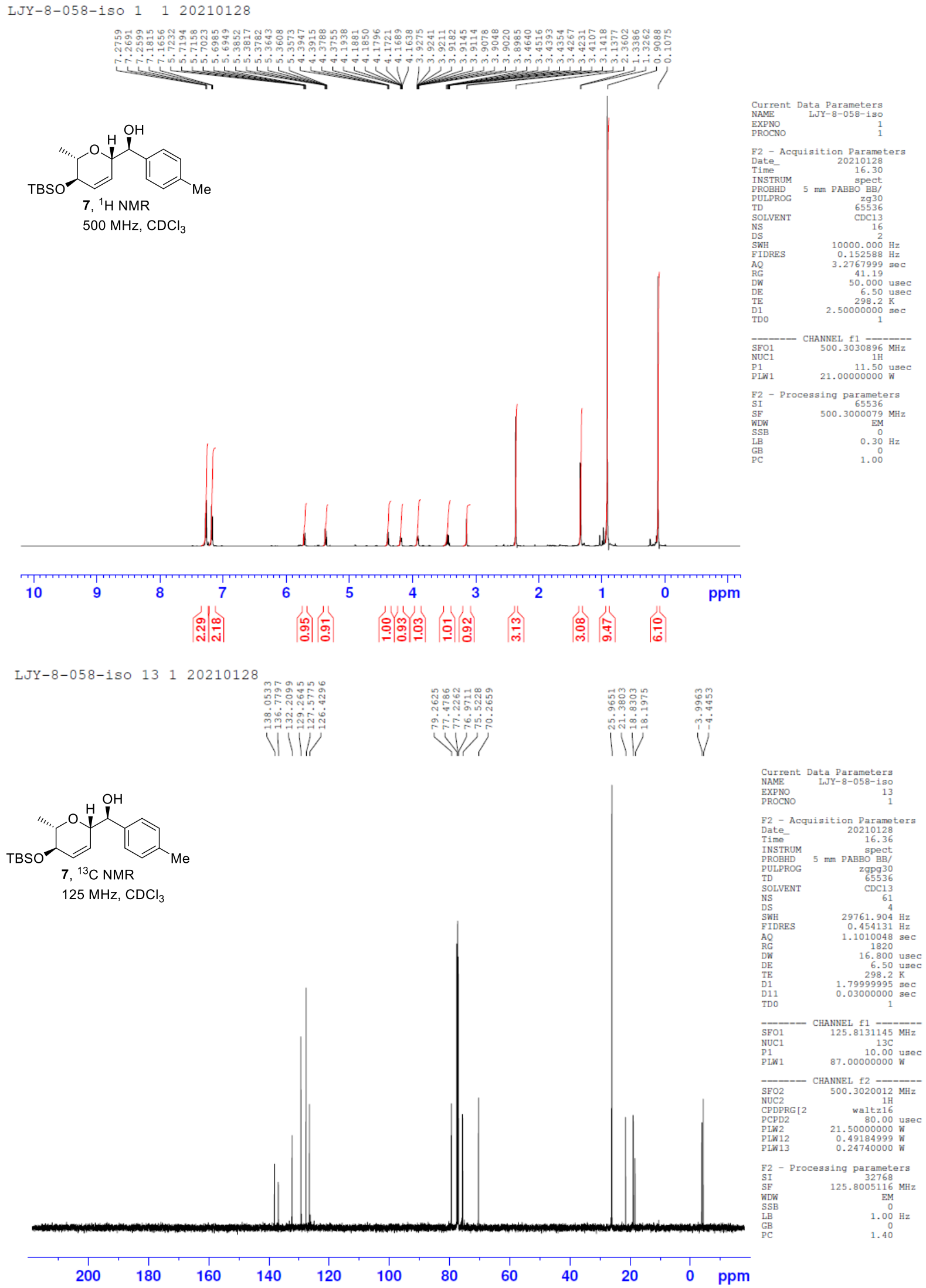



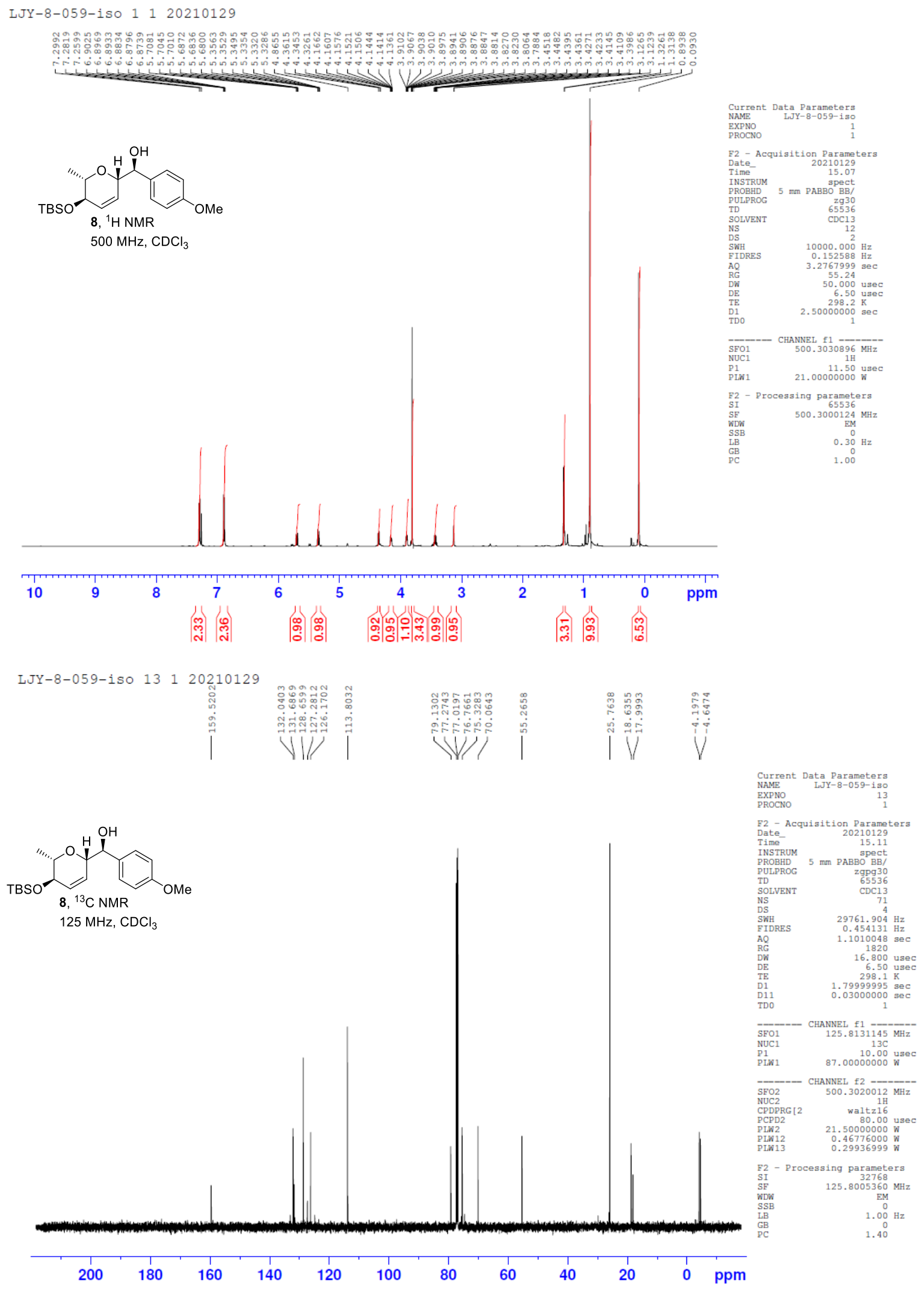

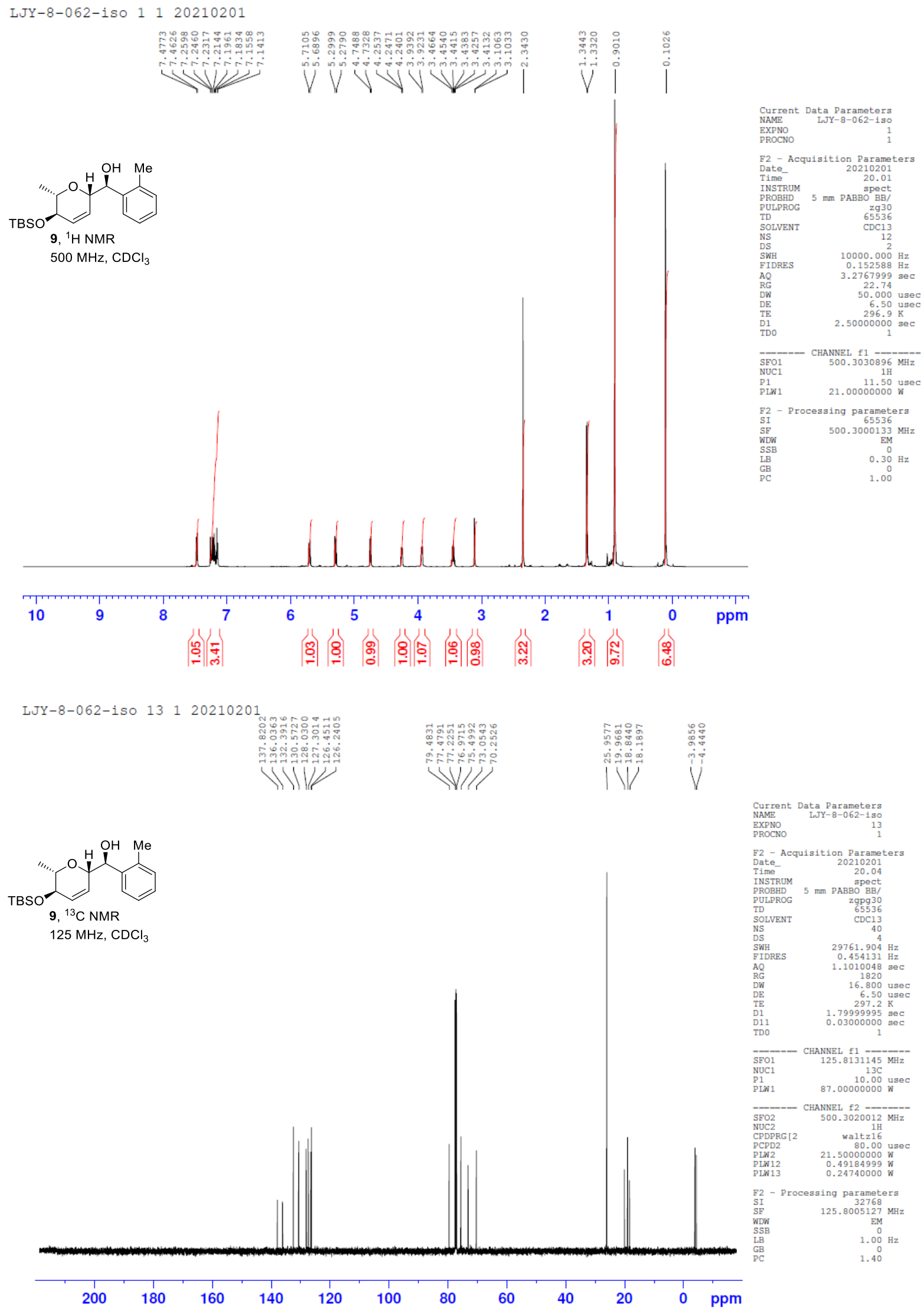

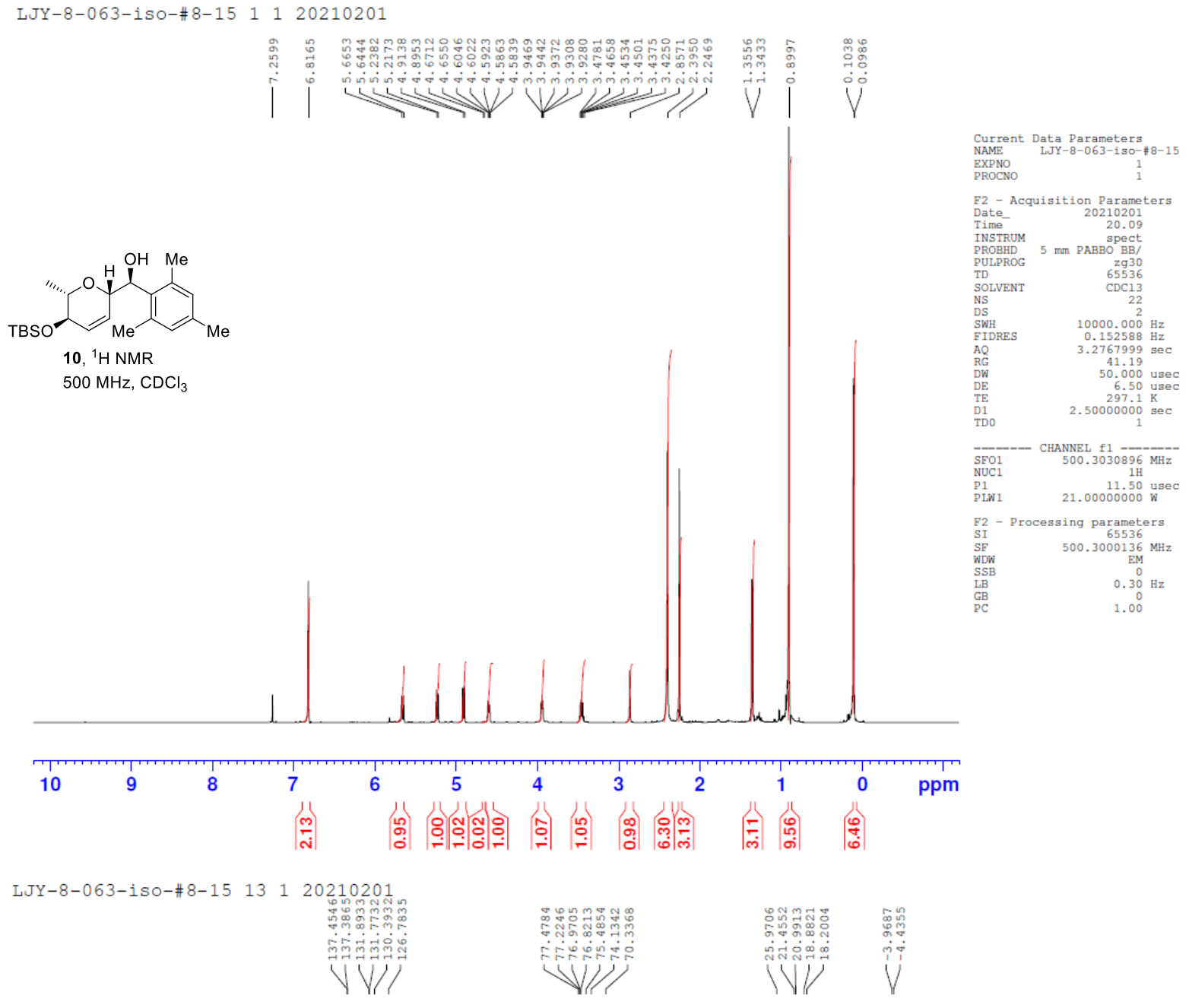

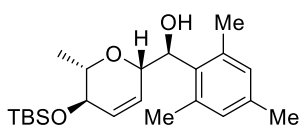

10, ${ }^{13} \mathrm{C}$ NMR

$125 \mathrm{MHz}, \mathrm{CDCl}_{3}$

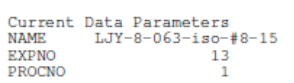

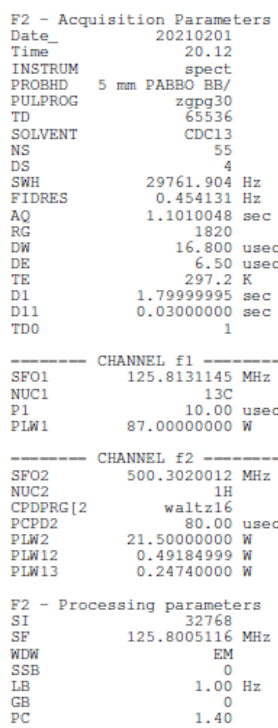



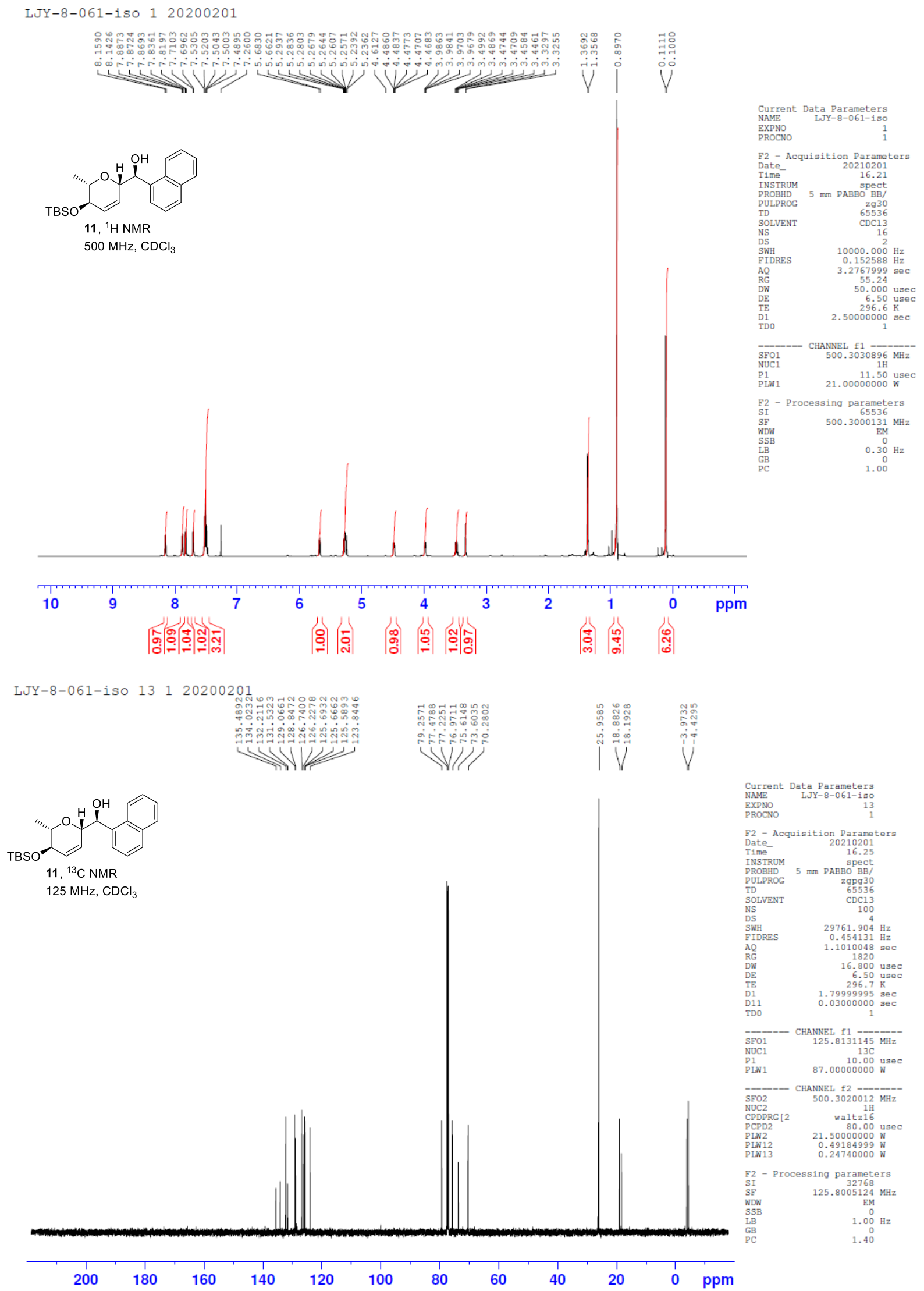


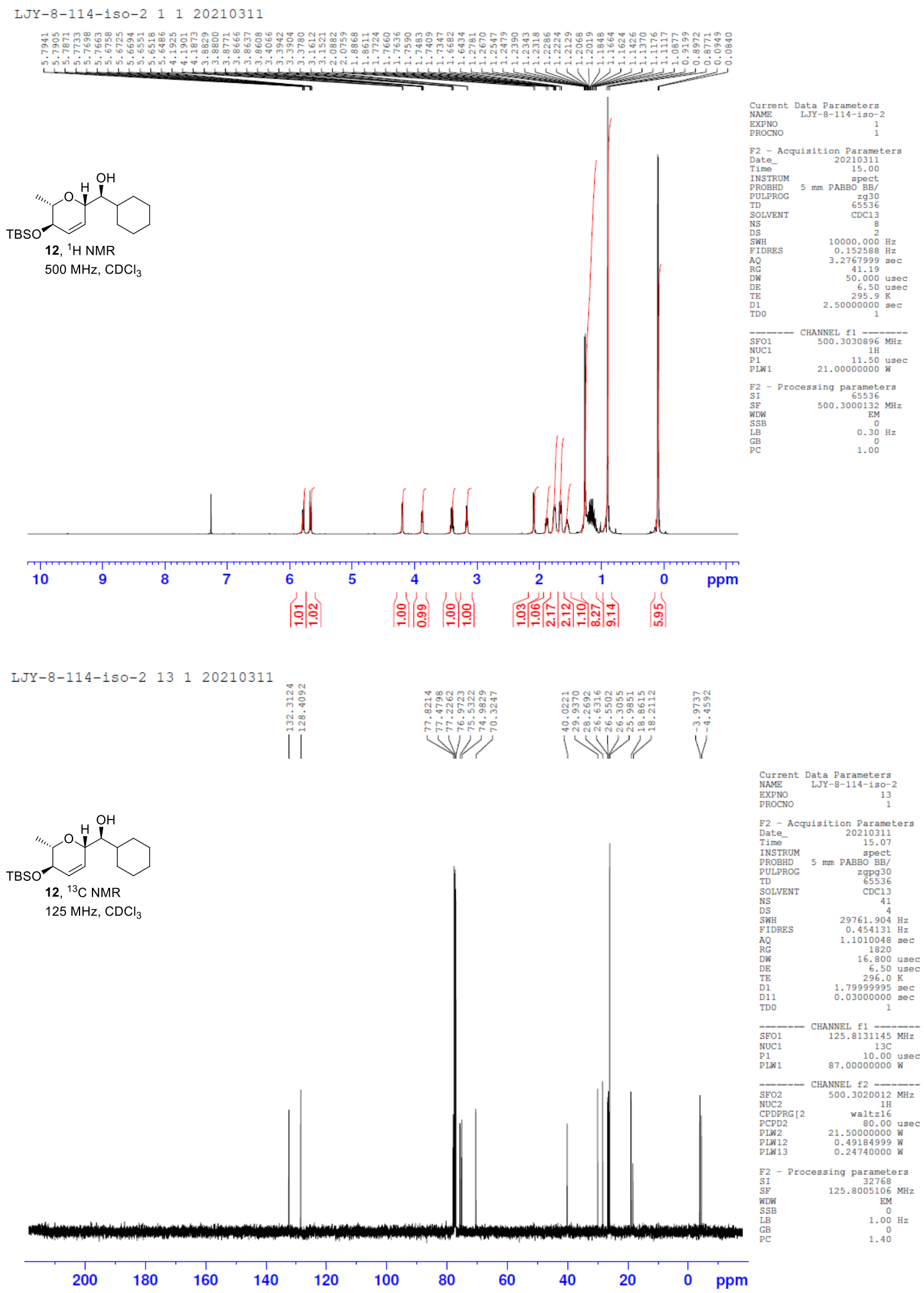




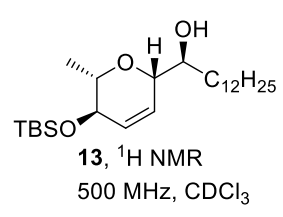

13, ${ }^{1} \mathrm{H}$ NMR
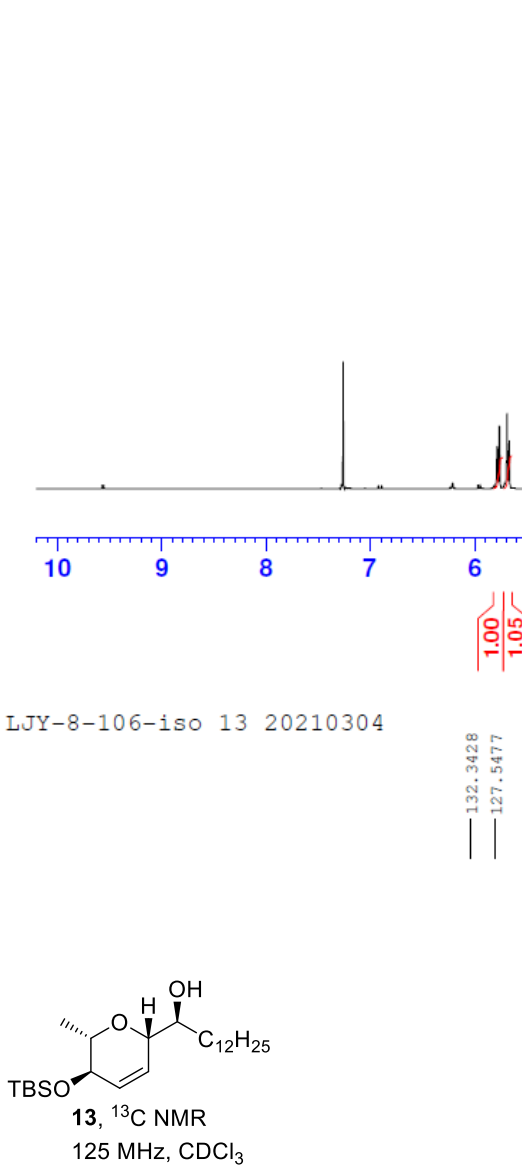

$125 \mathrm{MHz}, \mathrm{CDCl}_{3}$

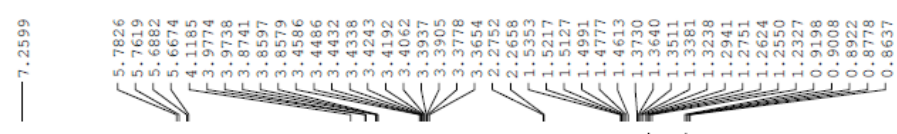

$\begin{array}{lr}\text { Current } & \text { Data Parameters } \\ \text { NAME } & \text { LUY-8-106-iso } \\ \text { EXPNO } & 1 \\ \text { PROCNO } & 1\end{array}$

F2 - Acquisition Parameters
Date- 20210304
Time-

\begin{tabular}{lr} 
Time & 11.56 \\
INSTRUM & spect \\
PROBDD & $5 \mathrm{~mm}$ PABBO BB/ \\
PULPROG & zag0 \\
PDPROG & 65536 \\
\hline
\end{tabular}

$\begin{array}{lr}\text { TD } & 65536 \\ \text { SOLVENT } & \text { CDC13 } \\ \text { NS } & 9\end{array}$

$\begin{array}{ll}\text { DS } & 10000.000 \mathrm{~Hz} \\ \text { SWH } & 0.152588 \mathrm{~Hz} \\ \text { FIDRES } & 3.2767999 \mathrm{gec}\end{array}$

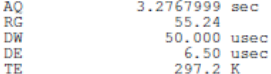

$\begin{array}{ll}\text { TE } & 297.2 \mathrm{~K} \\ \mathrm{D1} & 2.50000000 \mathrm{Kec}\end{array}$

\begin{tabular}{lr} 
& CHANNEL $\mathrm{f} 1-$ \\
\hline SEO1 & $500.3030896 \mathrm{MHz}$ \\
NUC1 & $1 \mathrm{H}$ \\
P1 & $11.50 \mathrm{usec}$ \\
PL1 1 & $21.00000000 \mathrm{~W}$ \\
F2 & processing parameters \\
SI & 65536 \\
SF & $500.3000122 \mathrm{MHz}$ \\
WDW & EM \\
SSB & 0 \\
LB & $0.30 \mathrm{~Hz}$ \\
GB & 0 \\
PC & 1.00
\end{tabular}

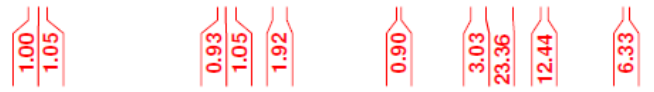

$\begin{array}{lr}\text { Current } & \text { Data Parameters } \\ \text { NAME } & \text { LJY-8-106-iso } \\ \text { EXPNO } & 13\end{array}$

NAME
EXPNO
PROCNO

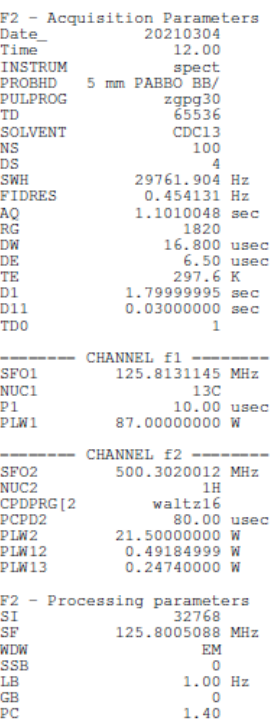

200

180

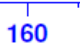

140

120

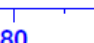

60

20

0 ppm 


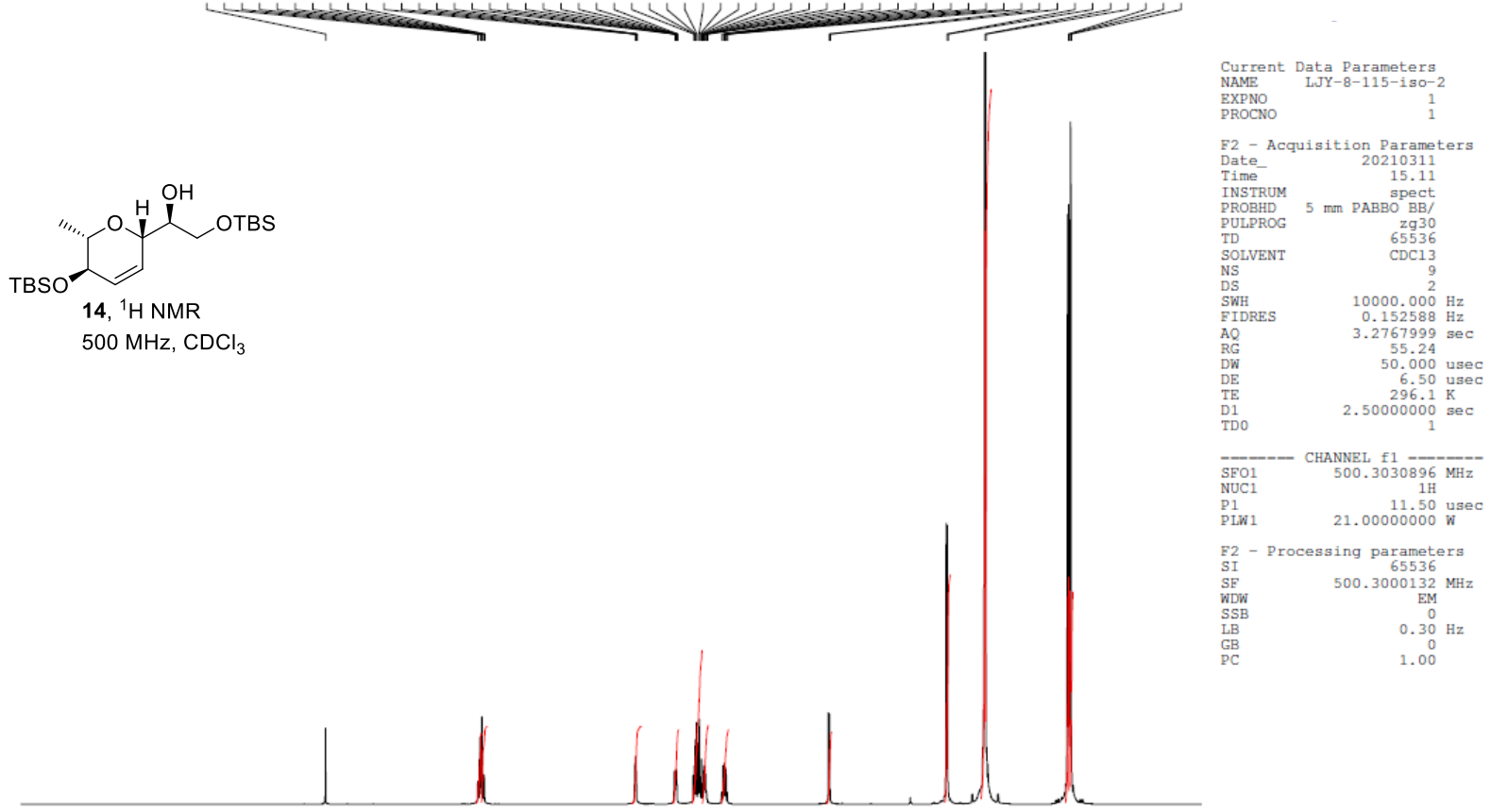

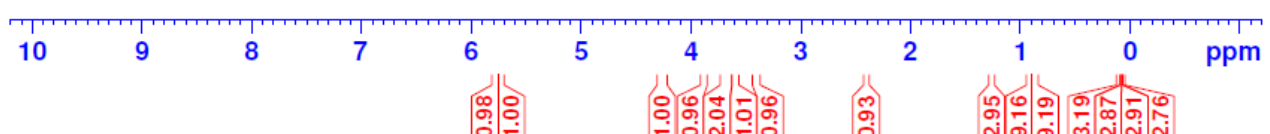
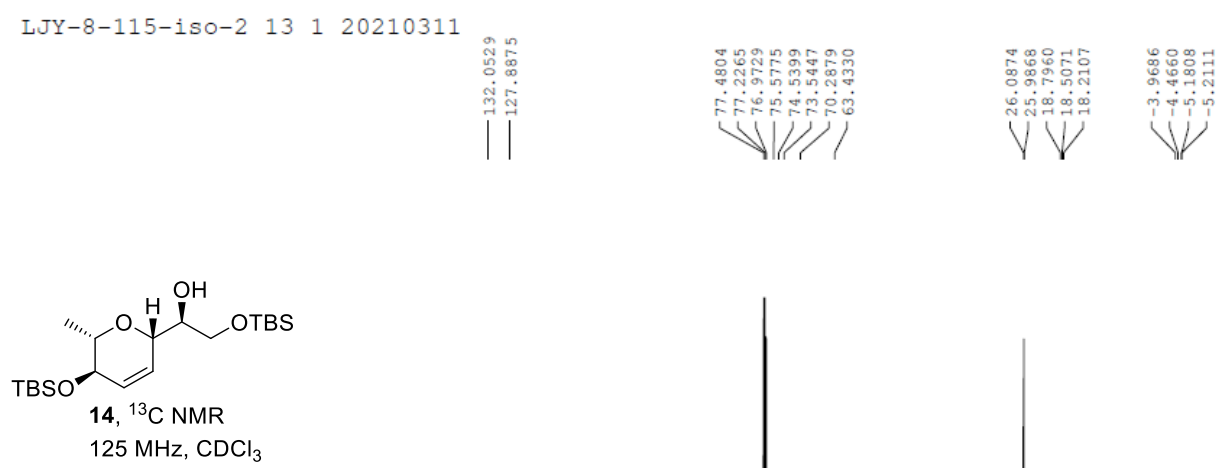

$125 \mathrm{MHz}, \mathrm{CDCl}_{3}$

$\begin{array}{lc}\text { Current } & \text { Data Parameters } \\ \text { NAME } & \text { LJY-8-115-13o-2 } \\ \text { EXPNO } & 13 \\ \text { PROCNO } & 1\end{array}$

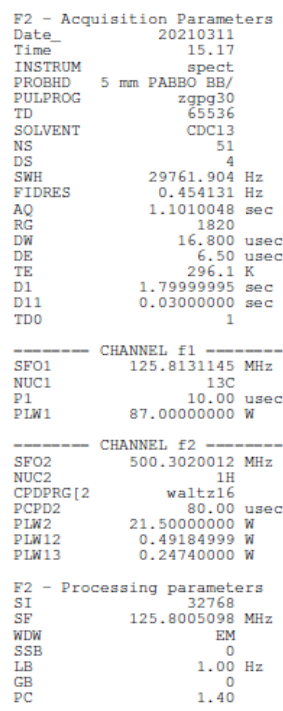



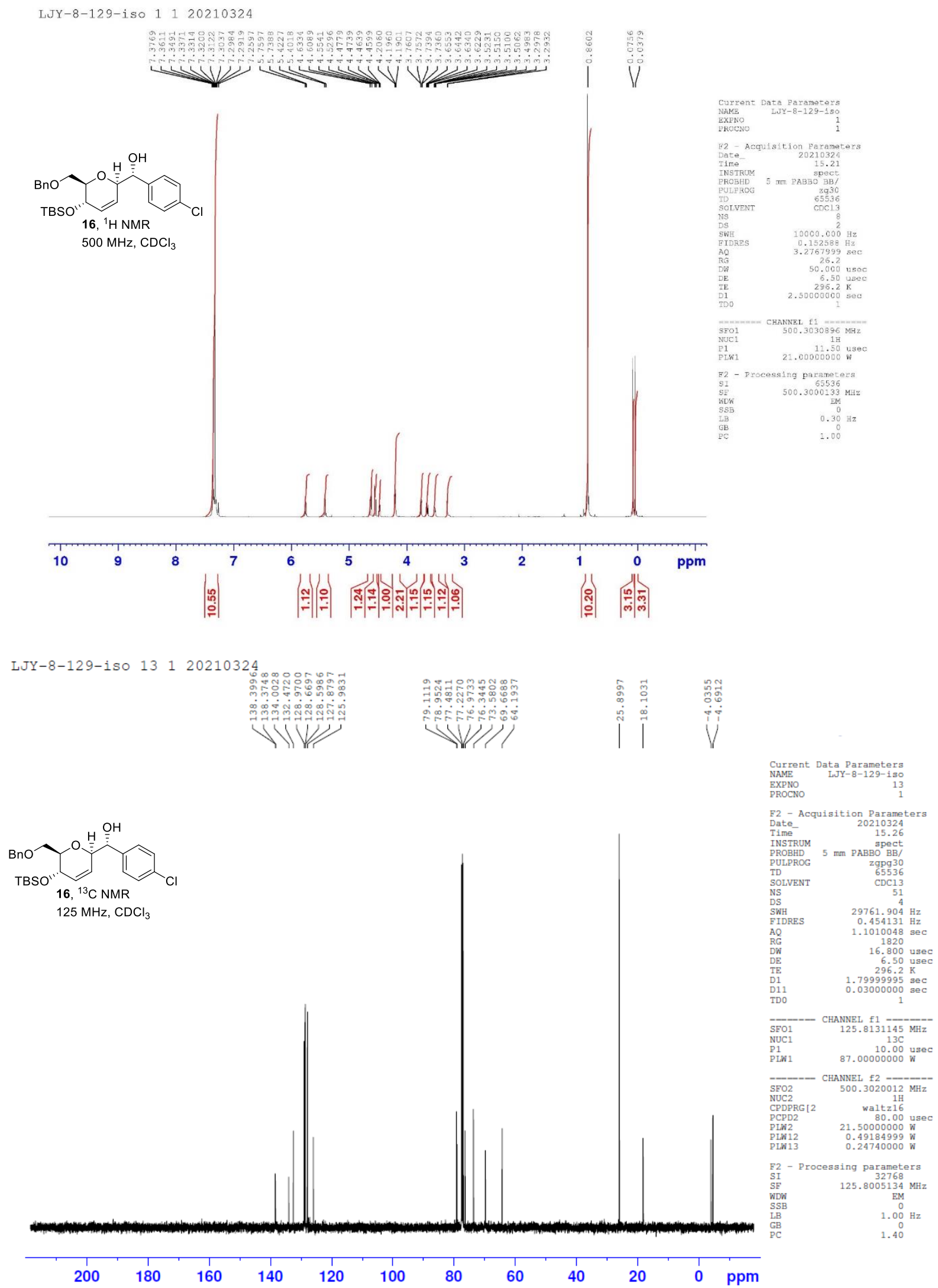

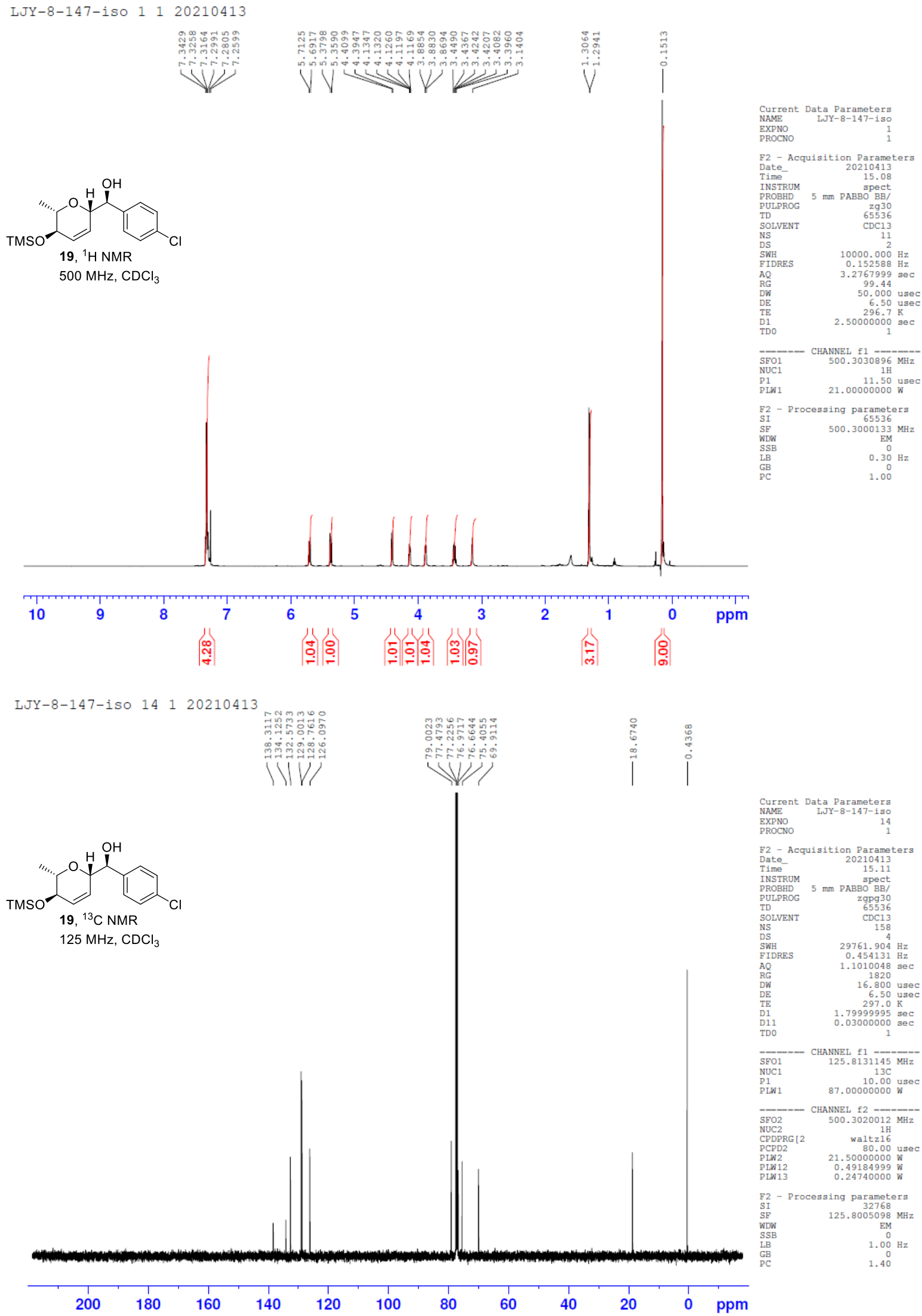

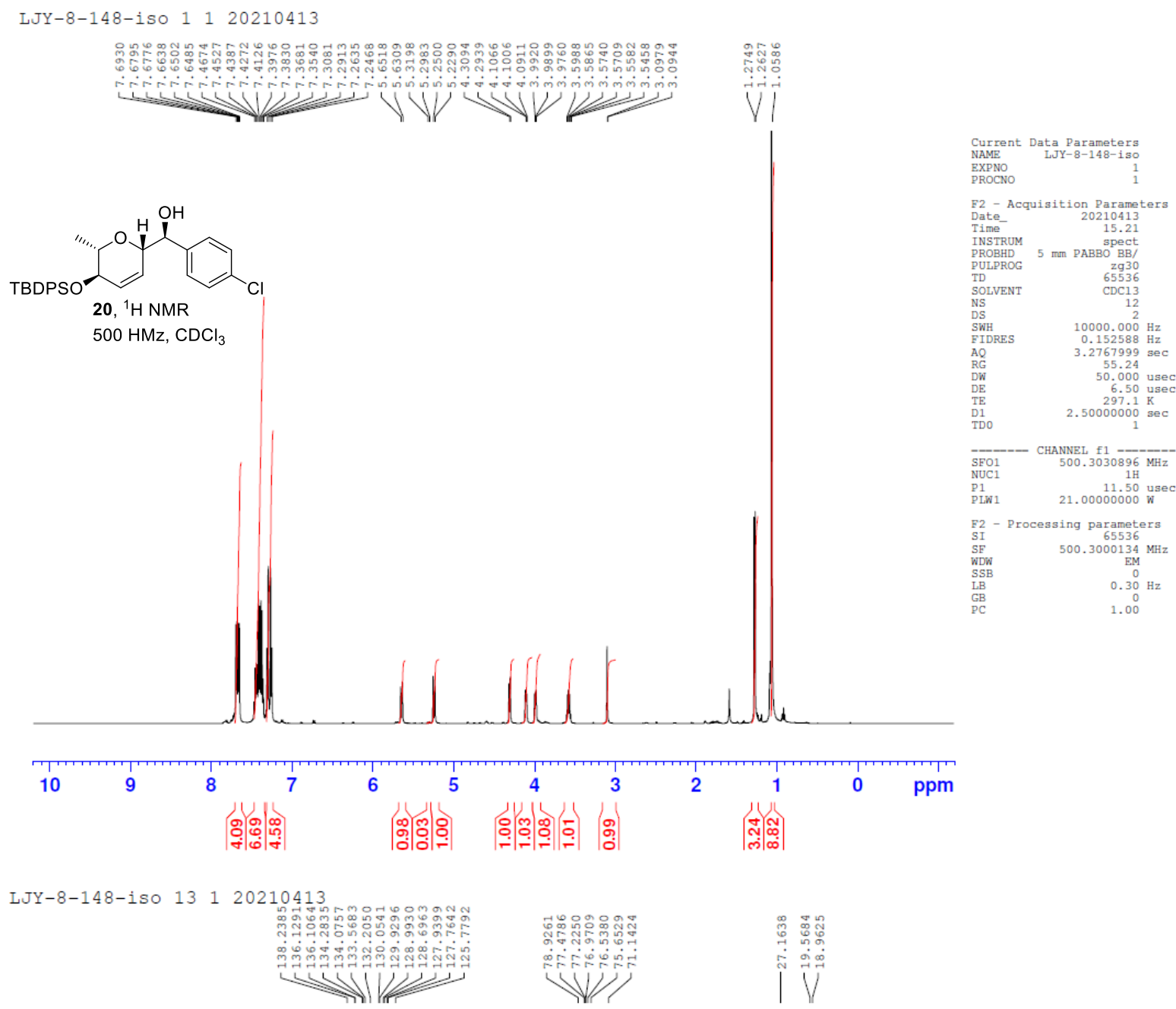

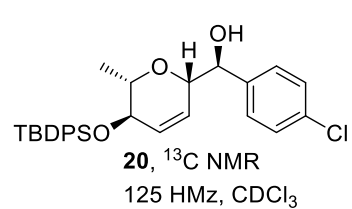

$125 \mathrm{HMz}, \mathrm{CDCl}_{3}$
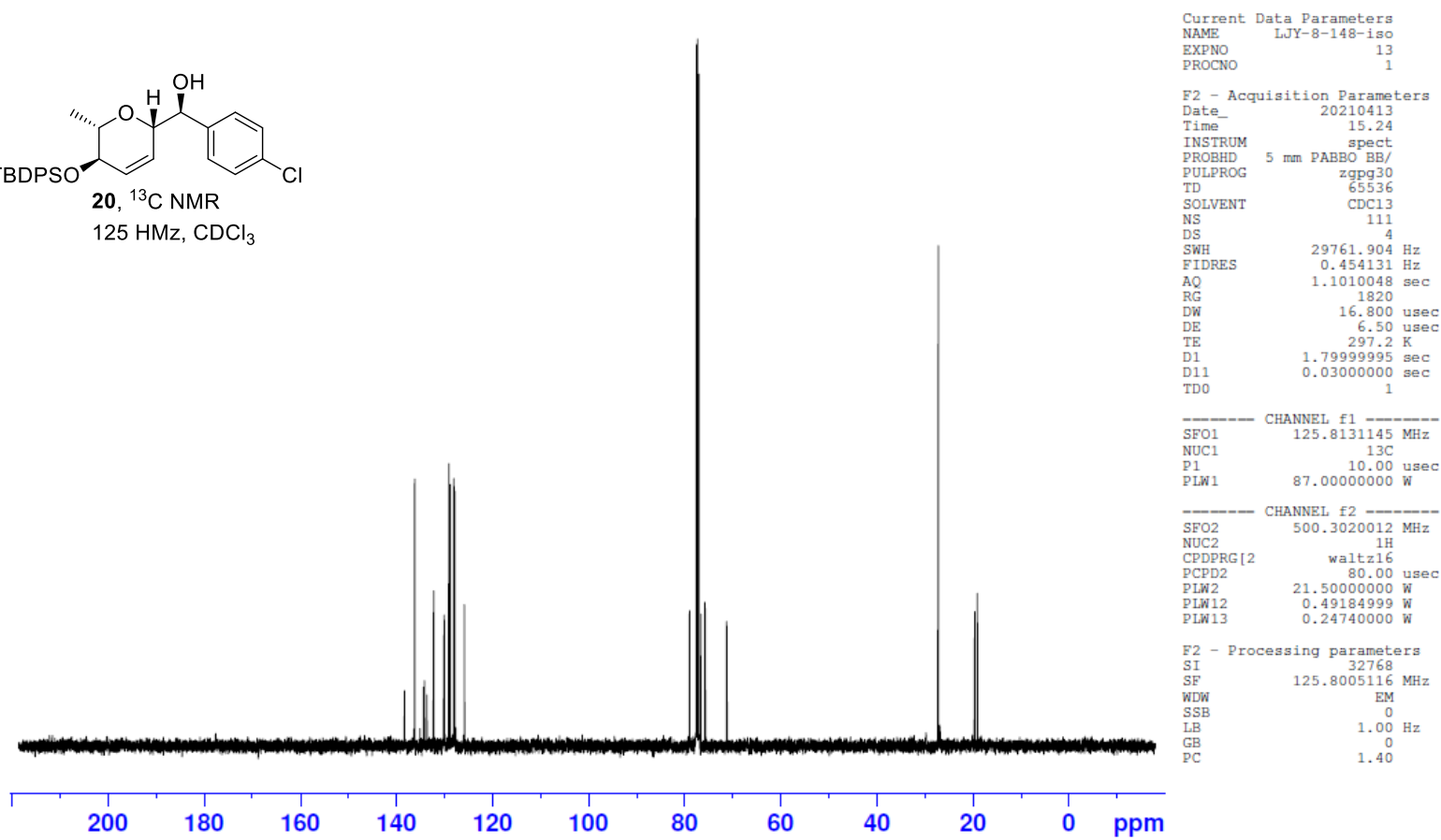

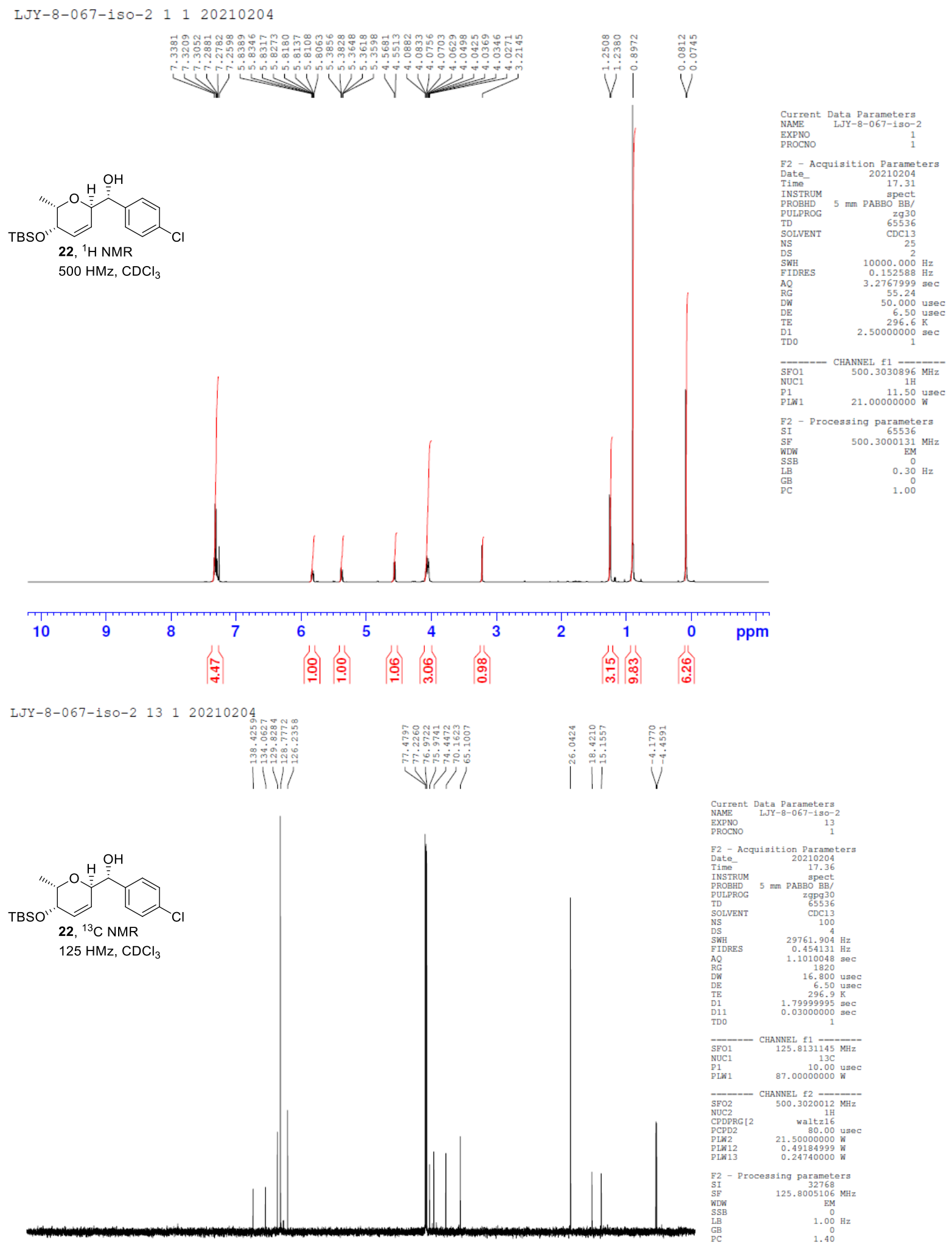

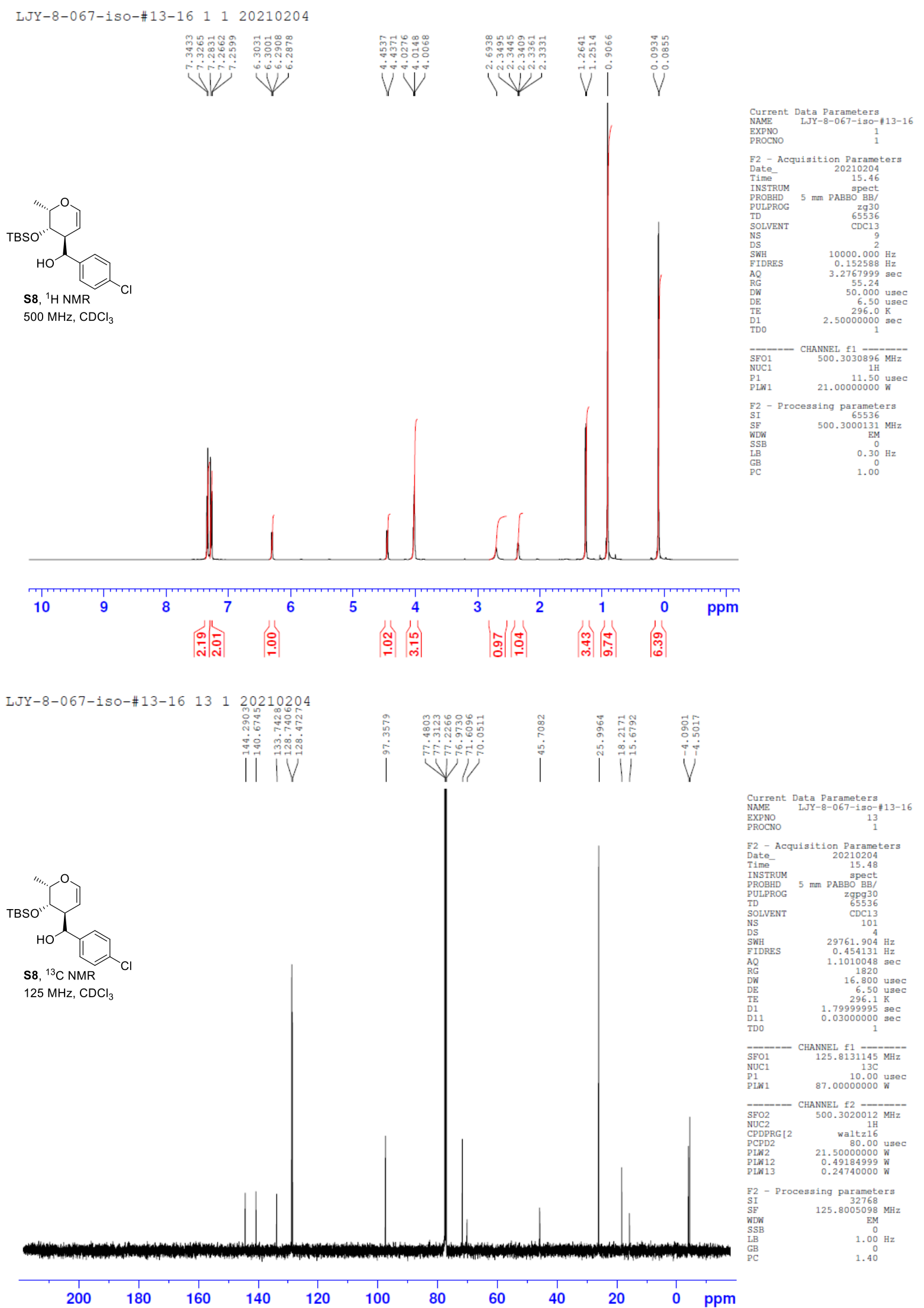

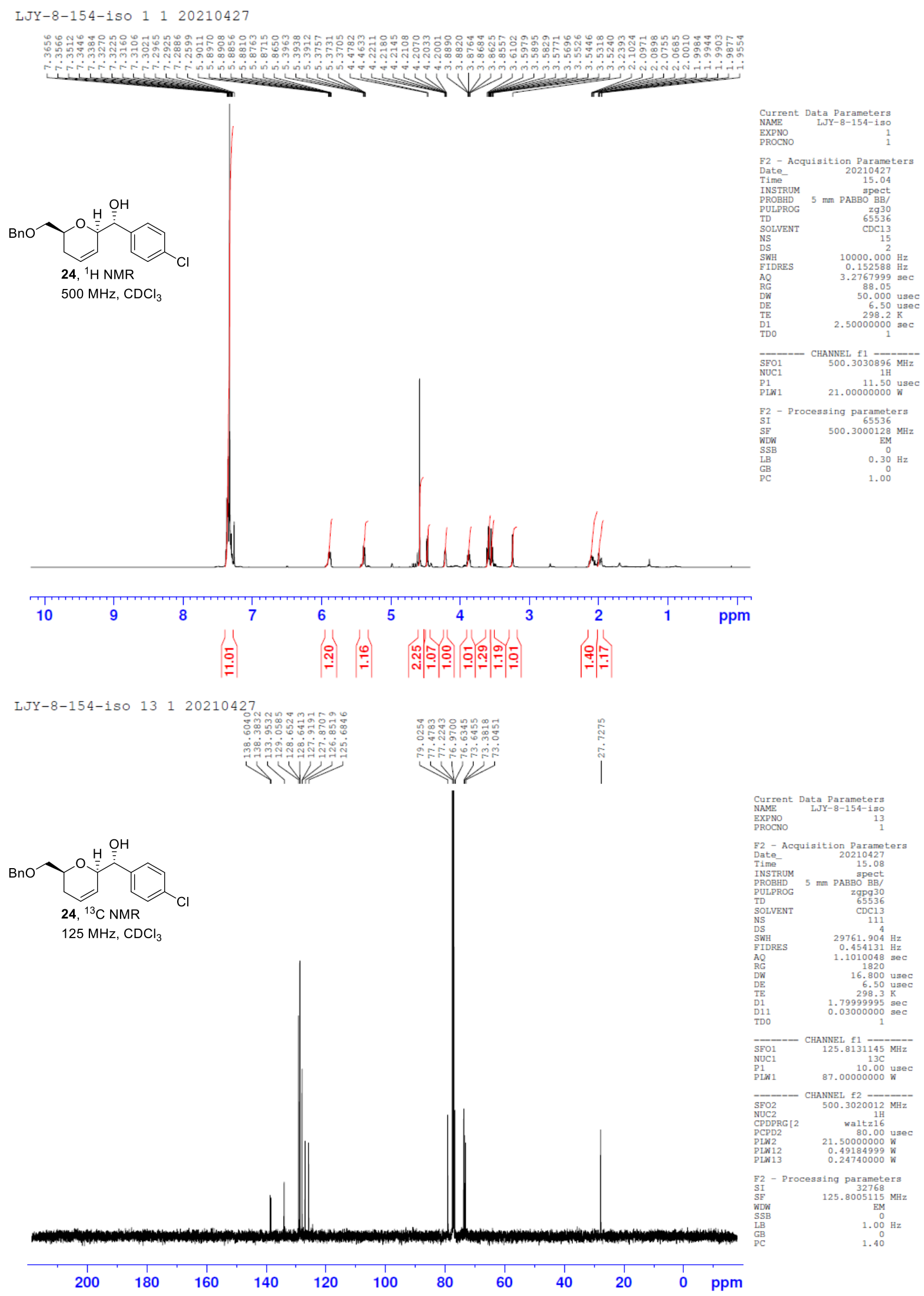


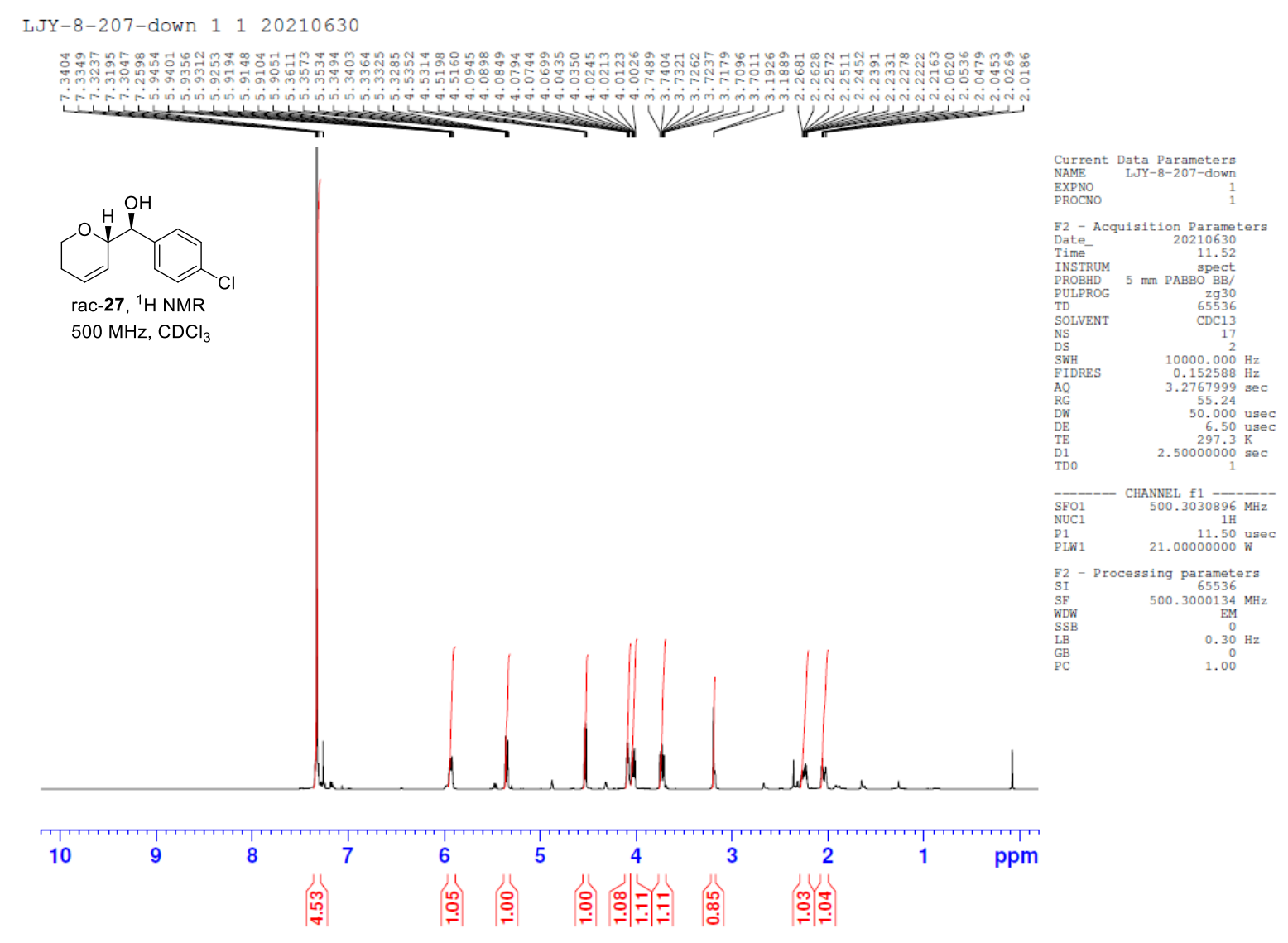

LJY-8-207-down 13120210630
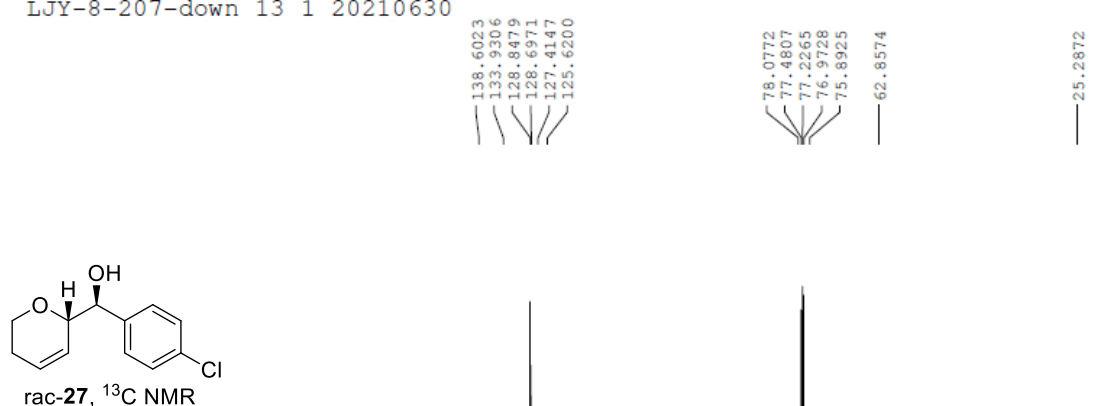

$125 \mathrm{MHz}, \mathrm{CDCl}_{3}$

$\begin{array}{lr}\text { Current } & \text { Data Parameters } \\ \text { NAME } & \text { IJY-8-207-down } \\ \text { EXPNO } & 13 \\ \text { PROCNO } & 1\end{array}$

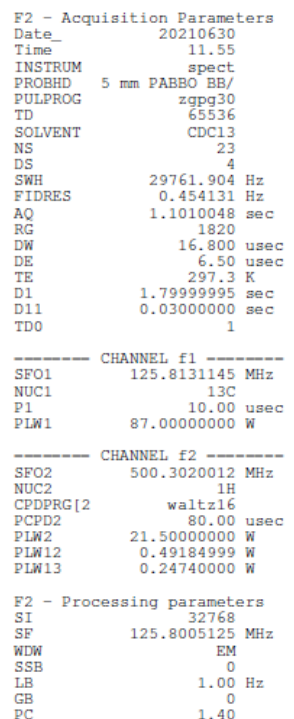

$200 \quad 180$

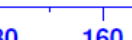

140

120

100

10

40

20

ppm 

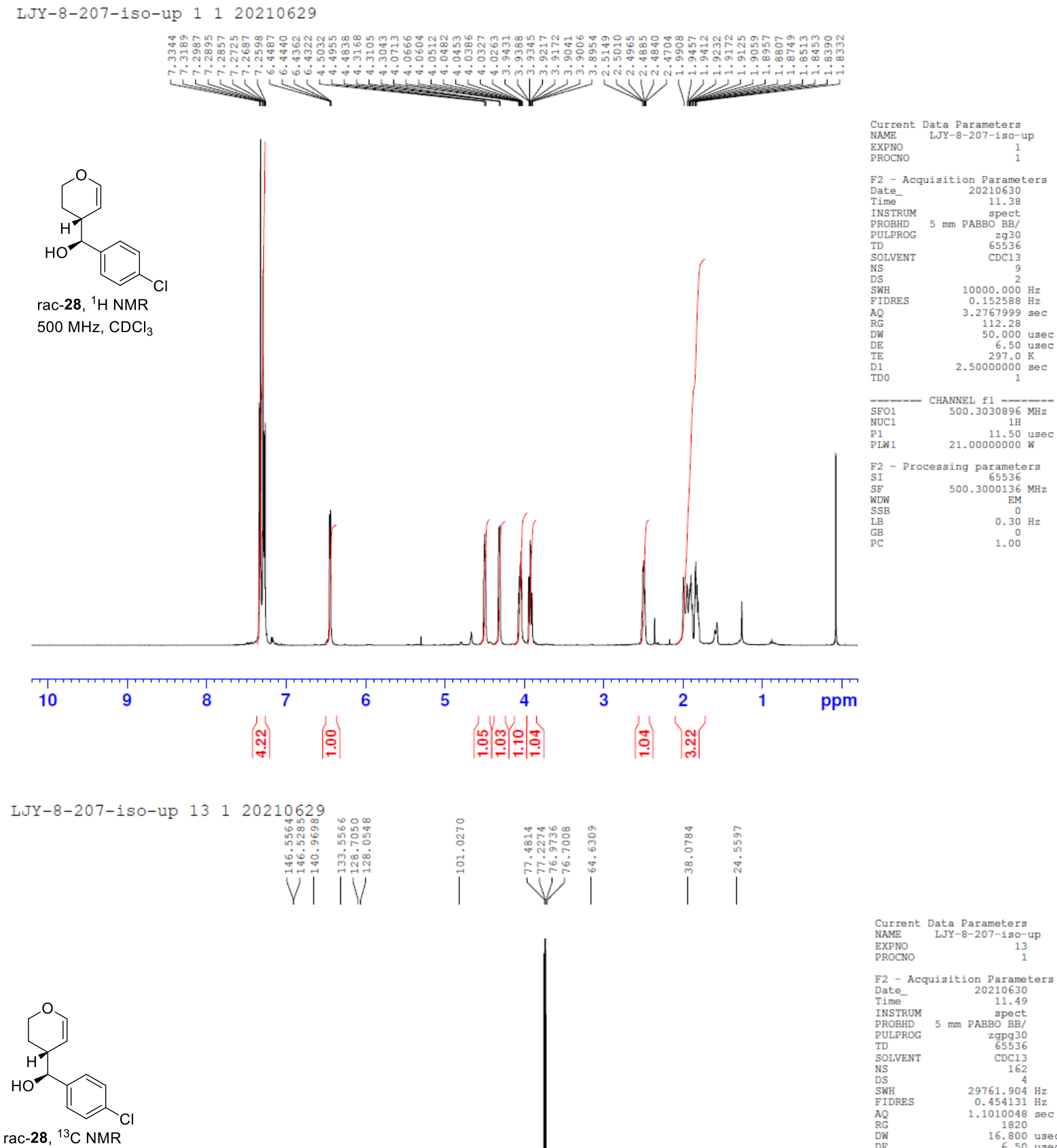

$125 \mathrm{MHz}, \mathrm{CDCl}_{3}$
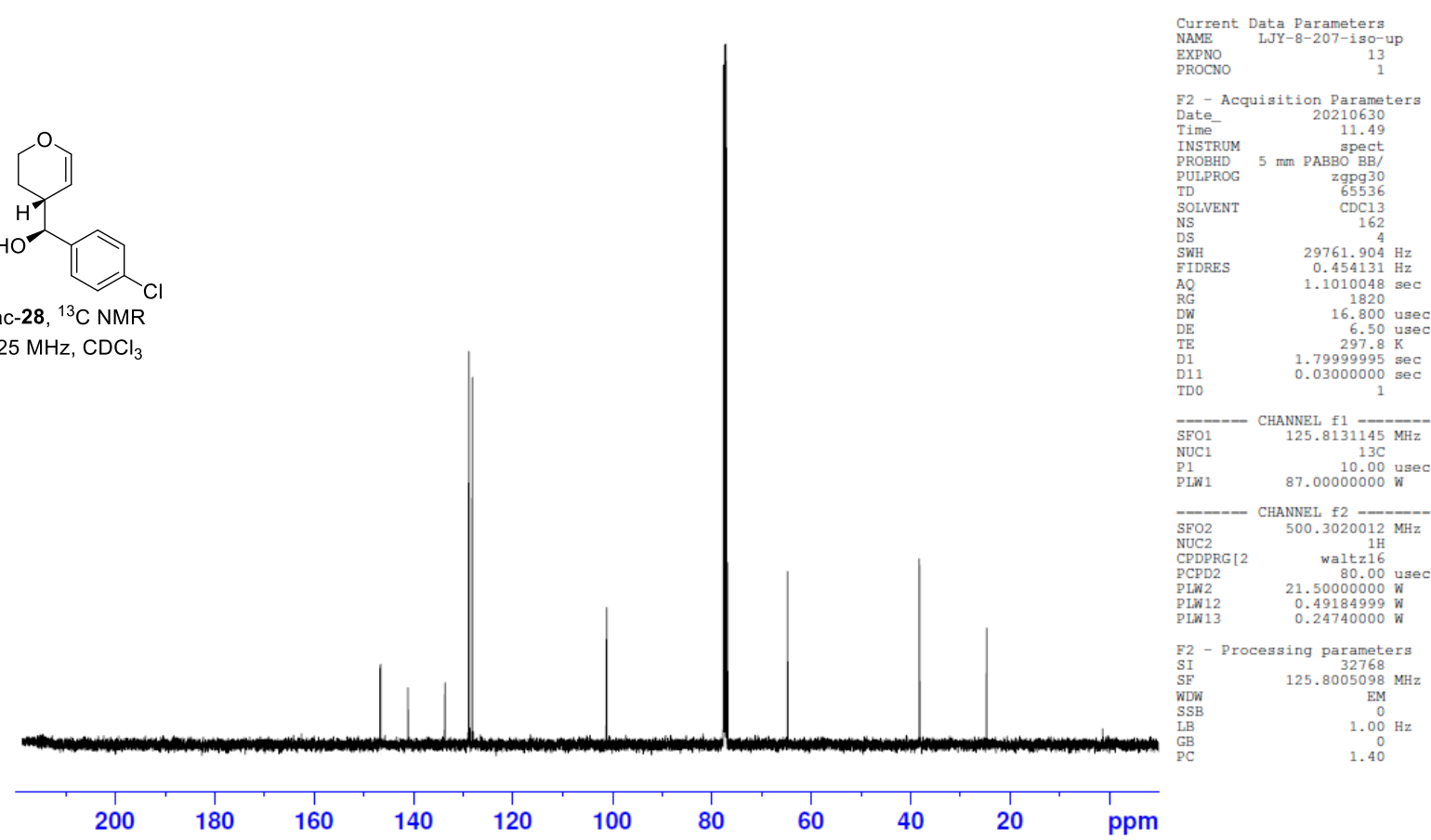
$\overbrace{C_{10} \mathrm{H}_{21}}^{\mathrm{OH}}$

30, ${ }^{1} \mathrm{H}$ NMR

$500 \mathrm{MHz}, \mathrm{CDCl}_{3}$
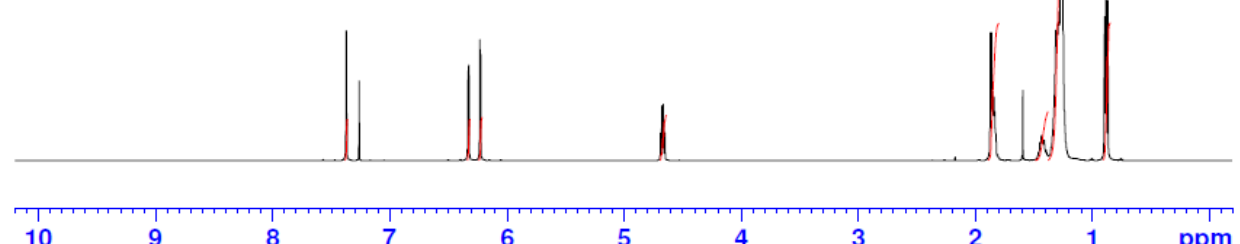

(웅

LJY-8-135-iso 13120210401

$\overbrace{\mathrm{C}_{10} \mathrm{H}_{21}}^{\mathrm{OH}}$

30, ${ }^{13} \mathrm{C}$ NMR

$125 \mathrm{MHz}, \mathrm{CDCl}_{3}$

ஸ્ڤ
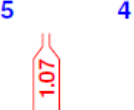

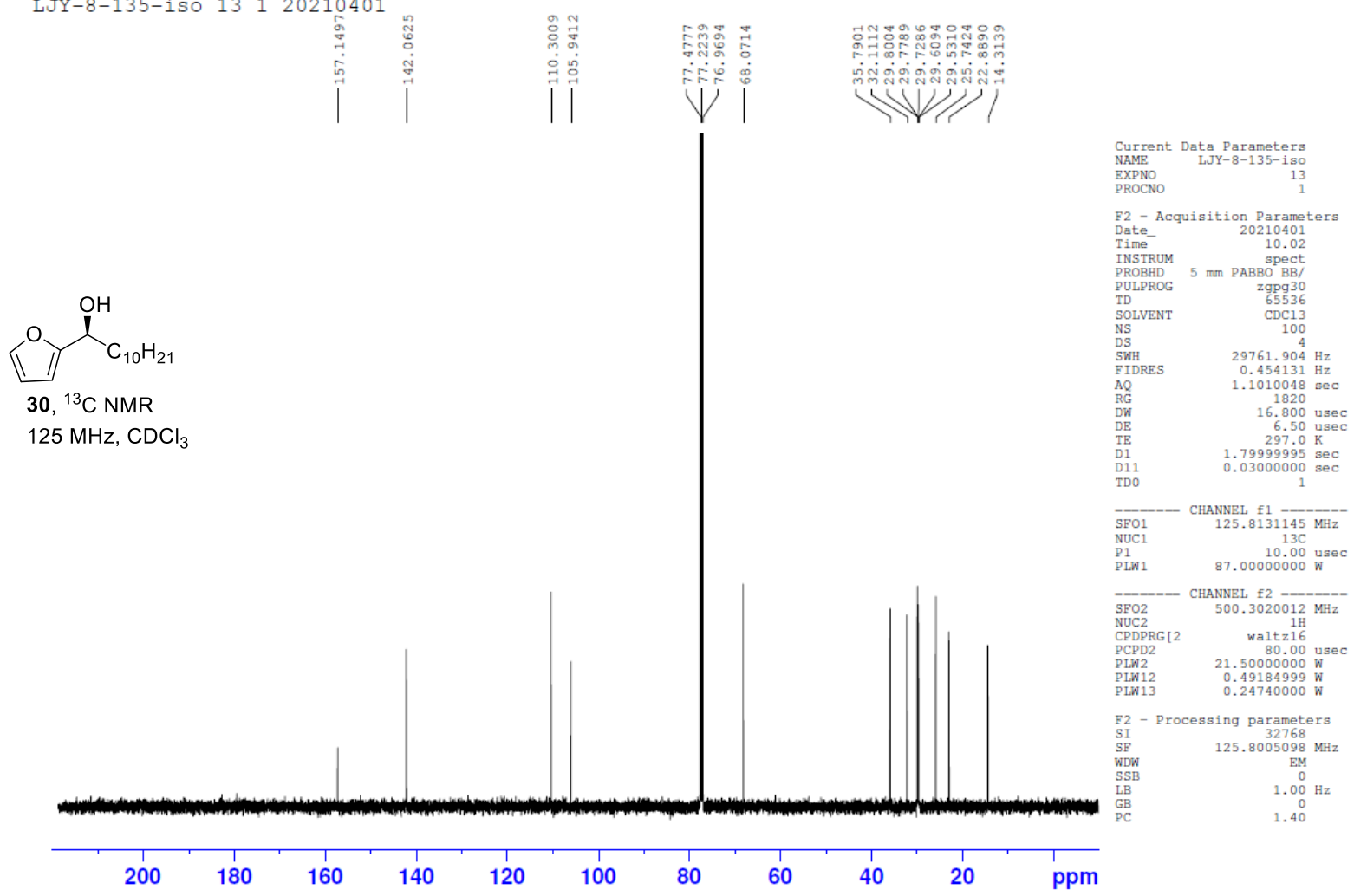

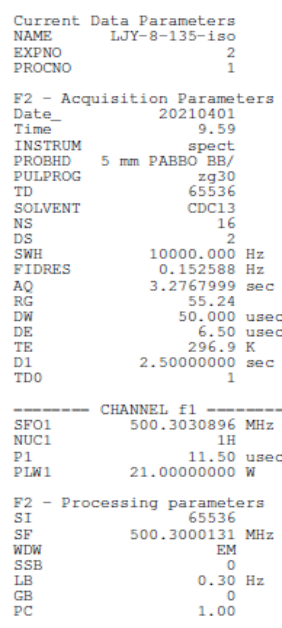



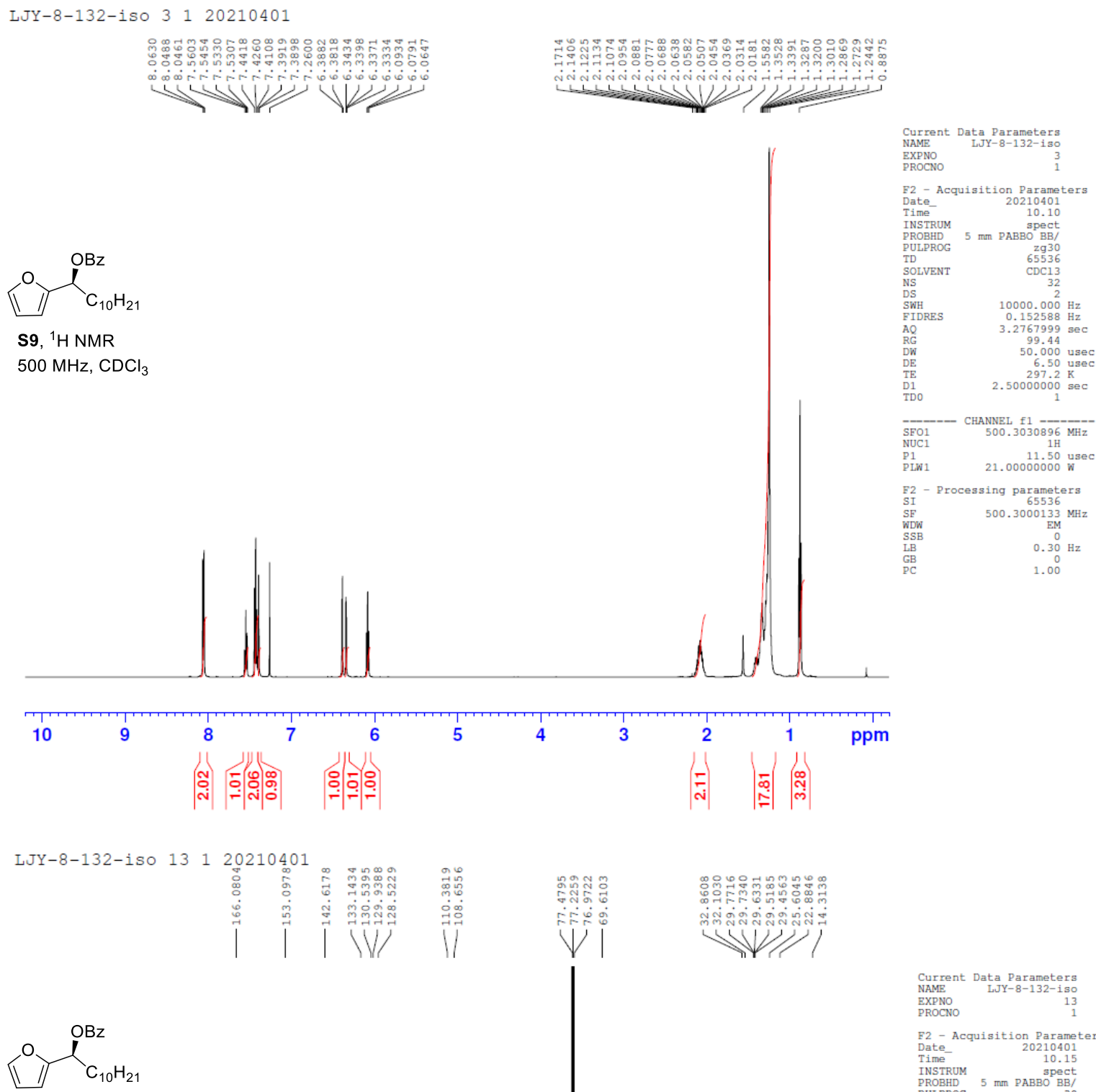

s9, ${ }^{13} \mathrm{C}$ NMR

$125 \mathrm{MHz} \mathrm{CDCl}_{3}$
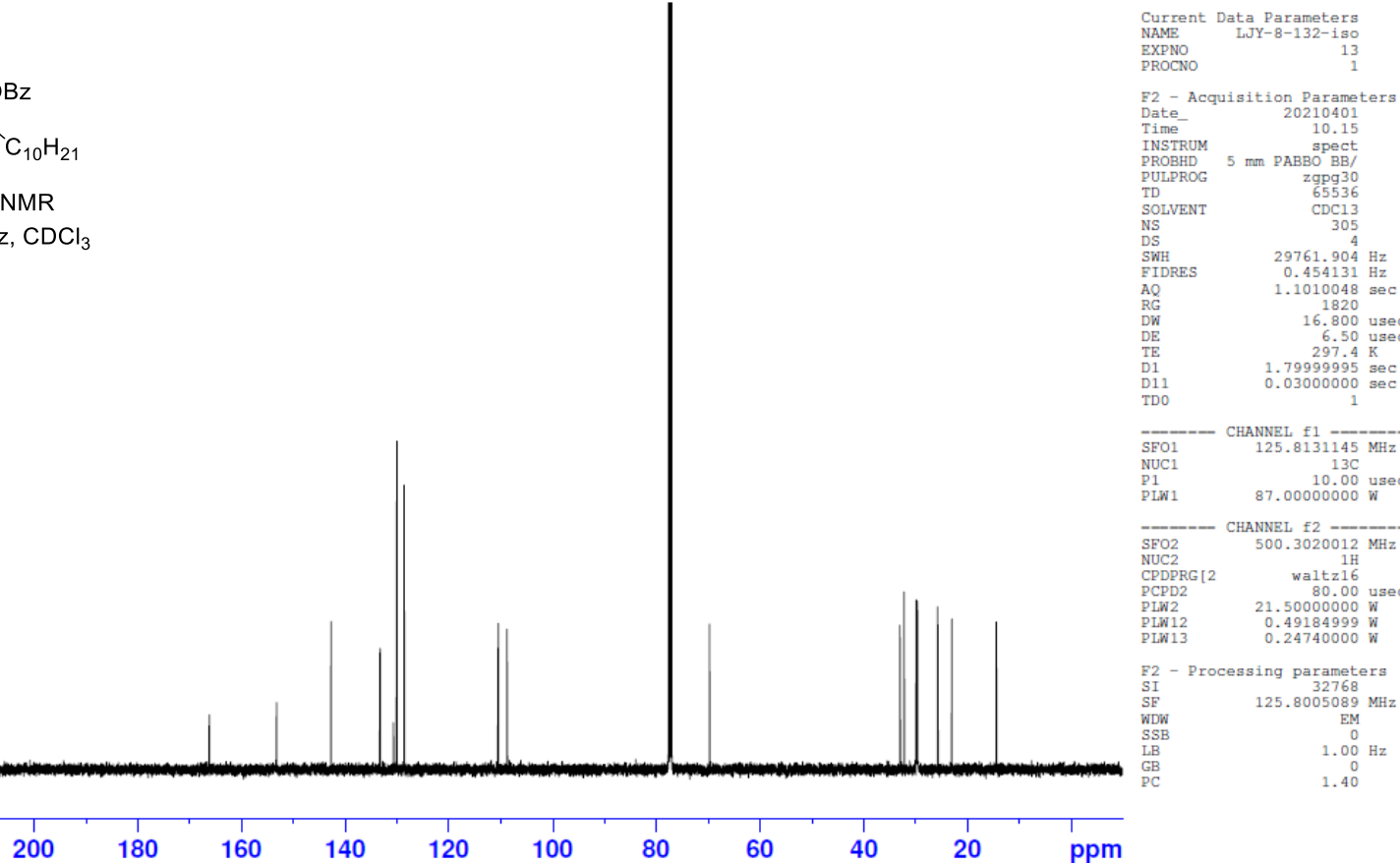

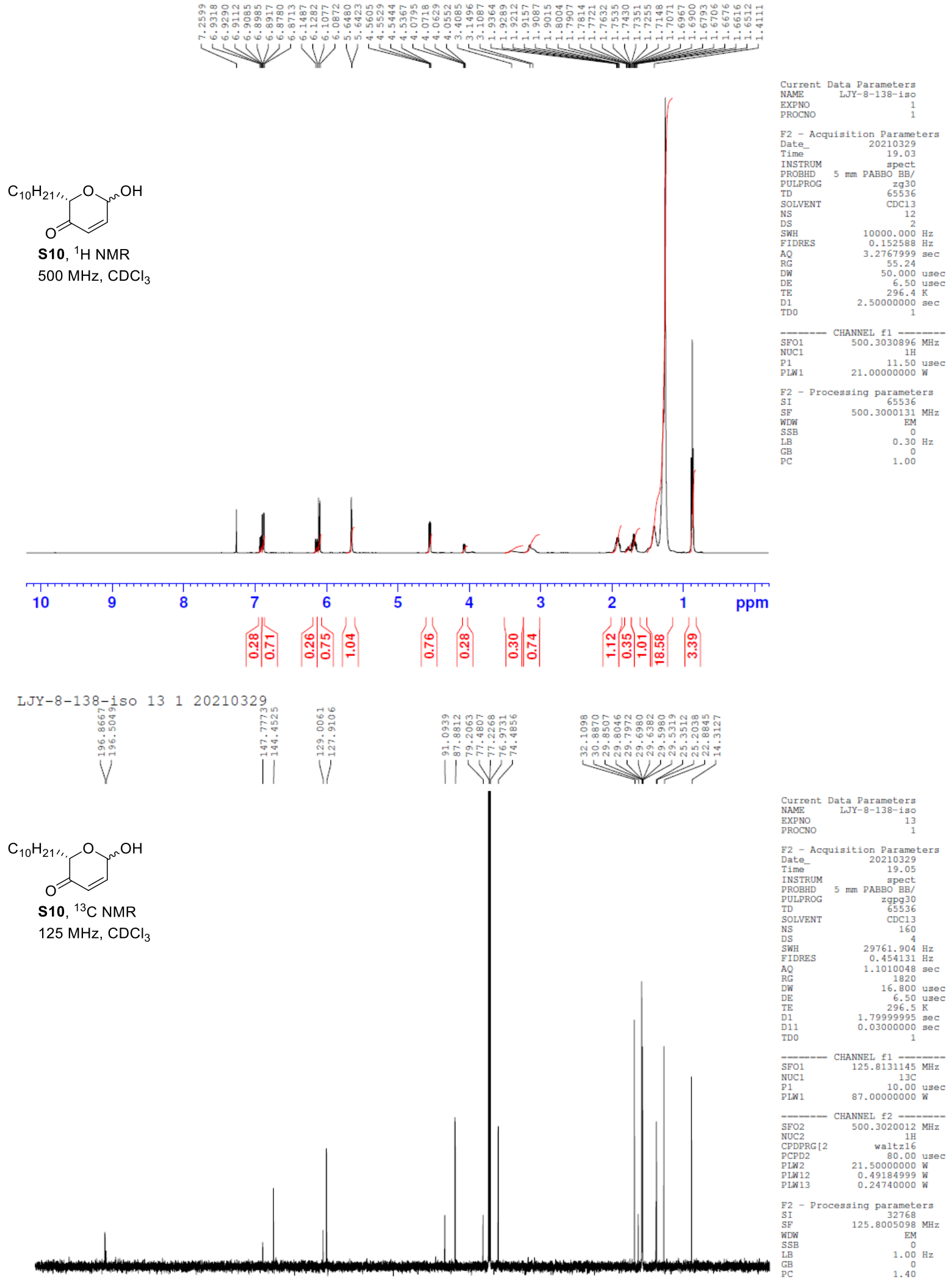

200

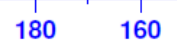

140

120

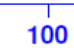

80

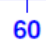

40

20

ppm 

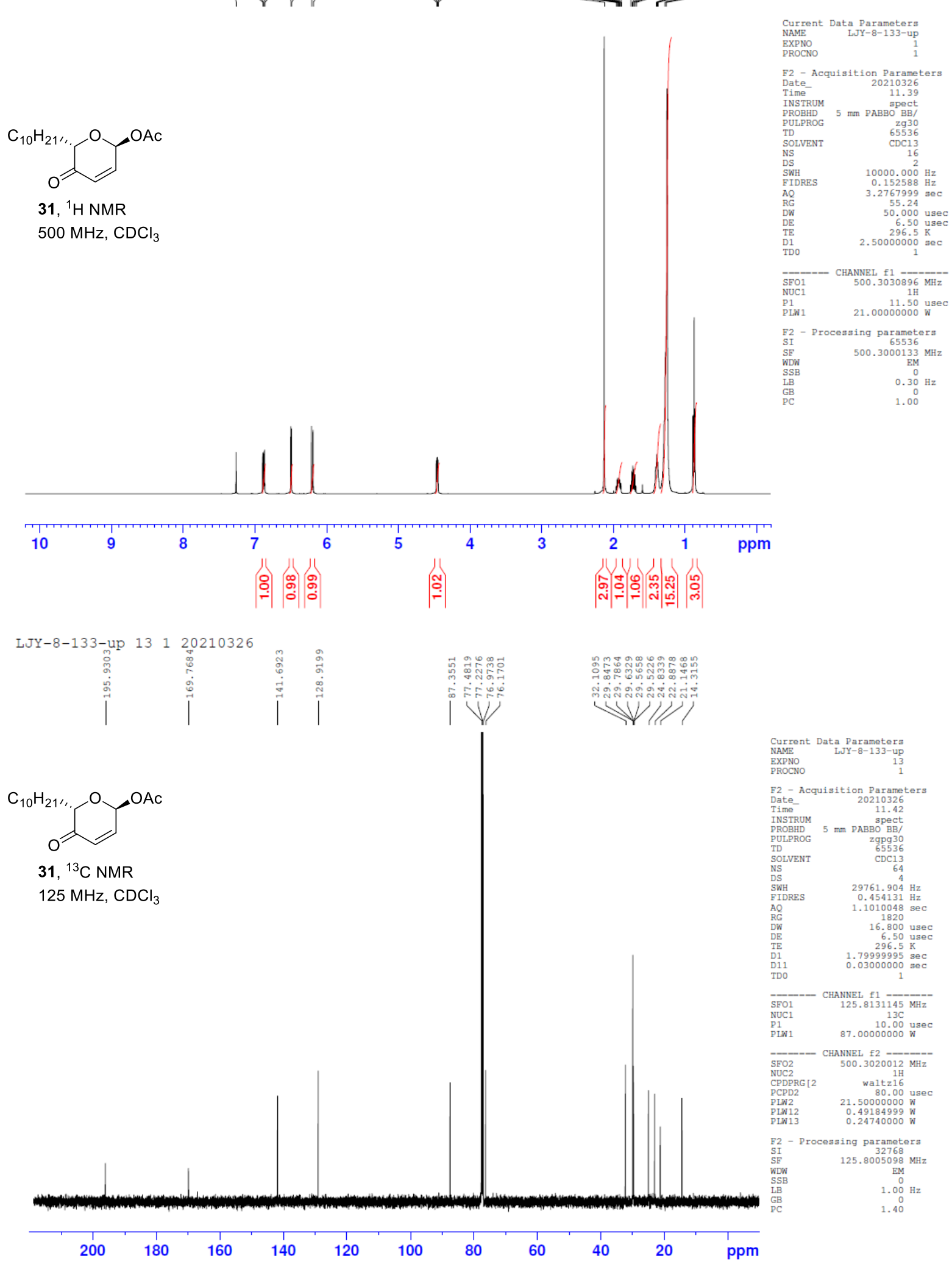


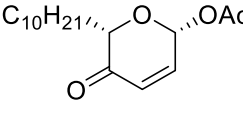

31b, ${ }^{1} \mathrm{H}$ NMR

$500 \mathrm{MHz}, \mathrm{CDCl}_{3}$
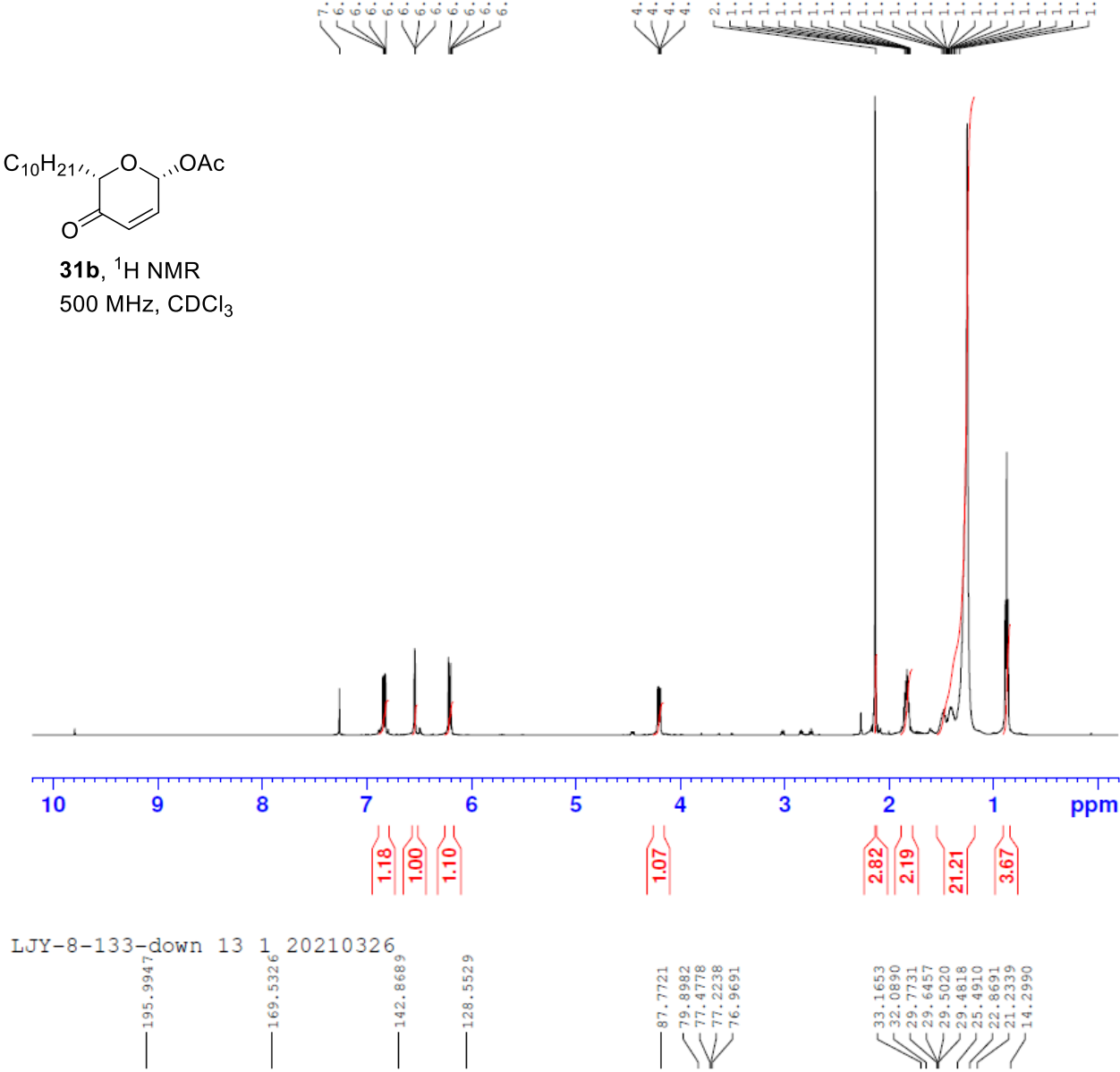

$\mathrm{C}_{10} \mathrm{H}_{21}$, $\mathrm{O}, . \mathrm{OAC}$

31b, ${ }^{13} \mathrm{C}$ NMR

$125 \mathrm{MHz}, \mathrm{CDCl}_{3}$
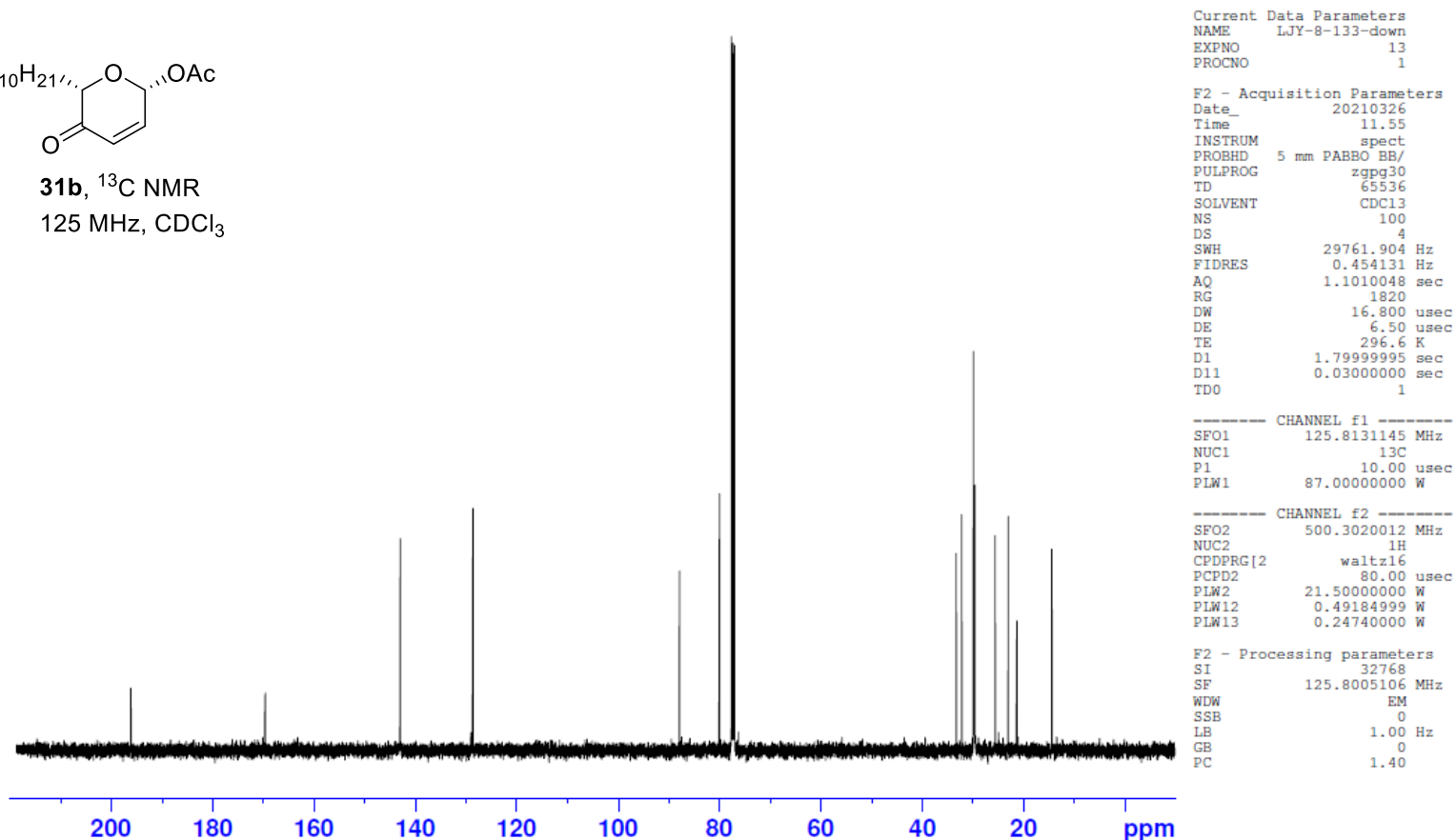

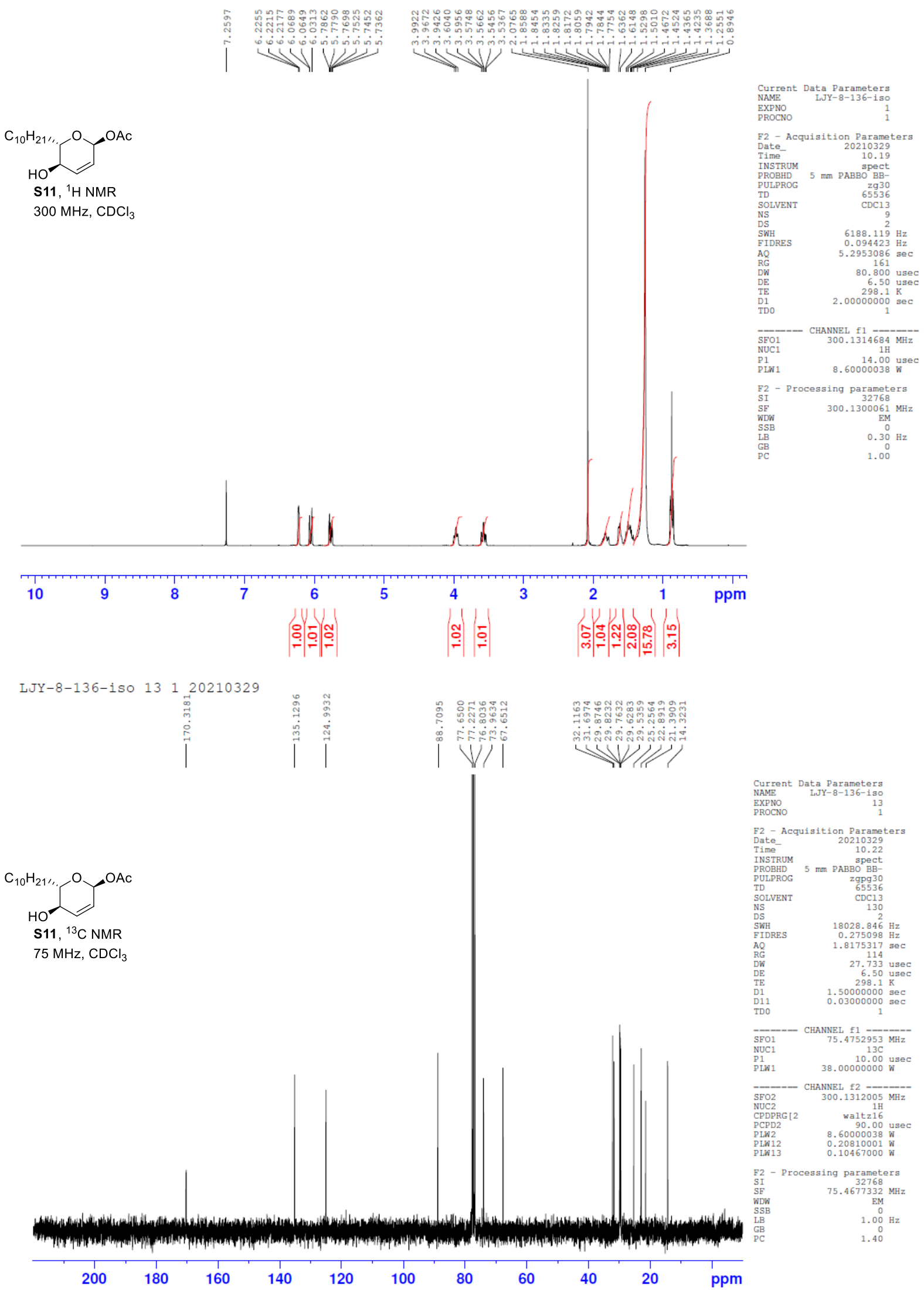

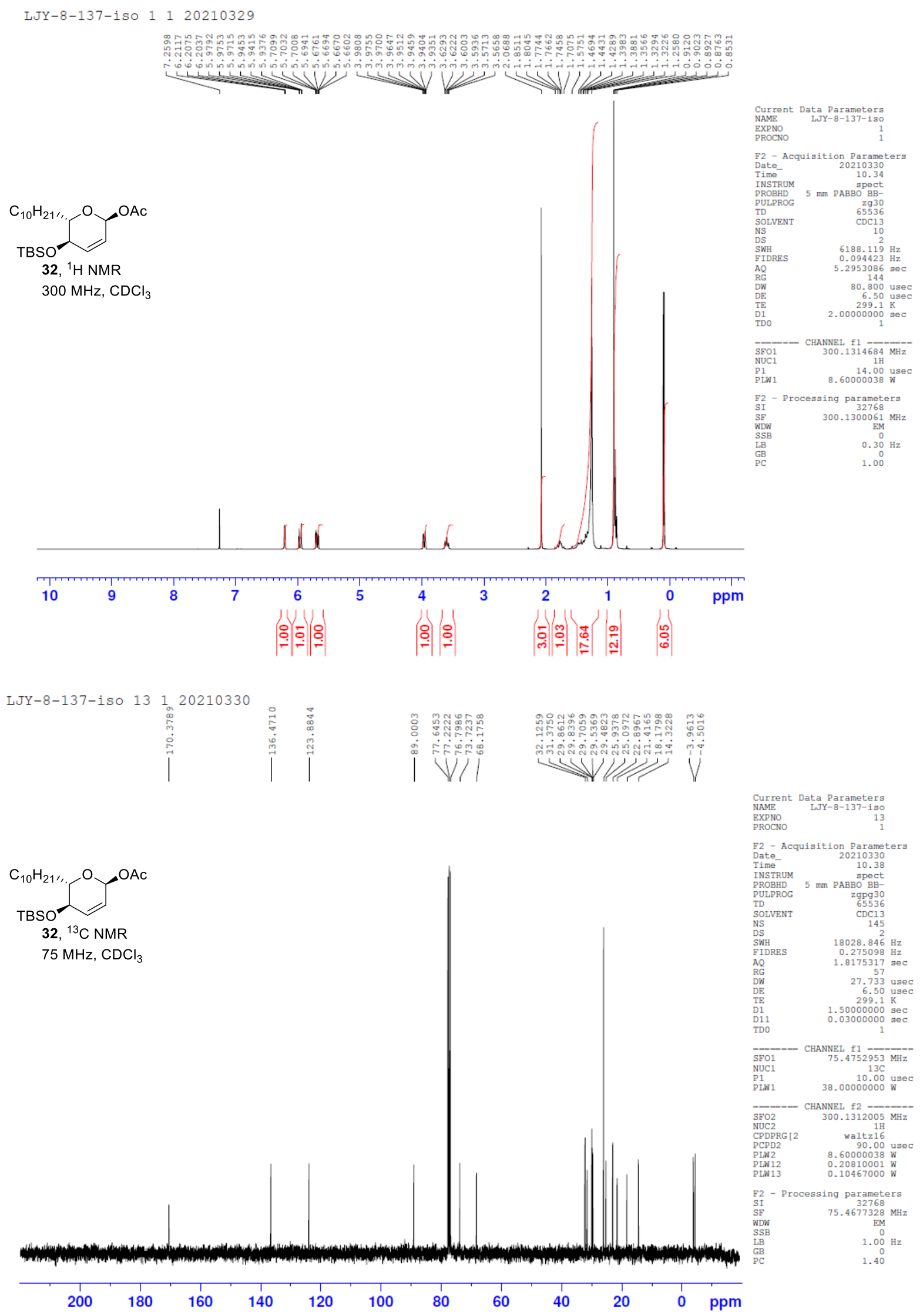


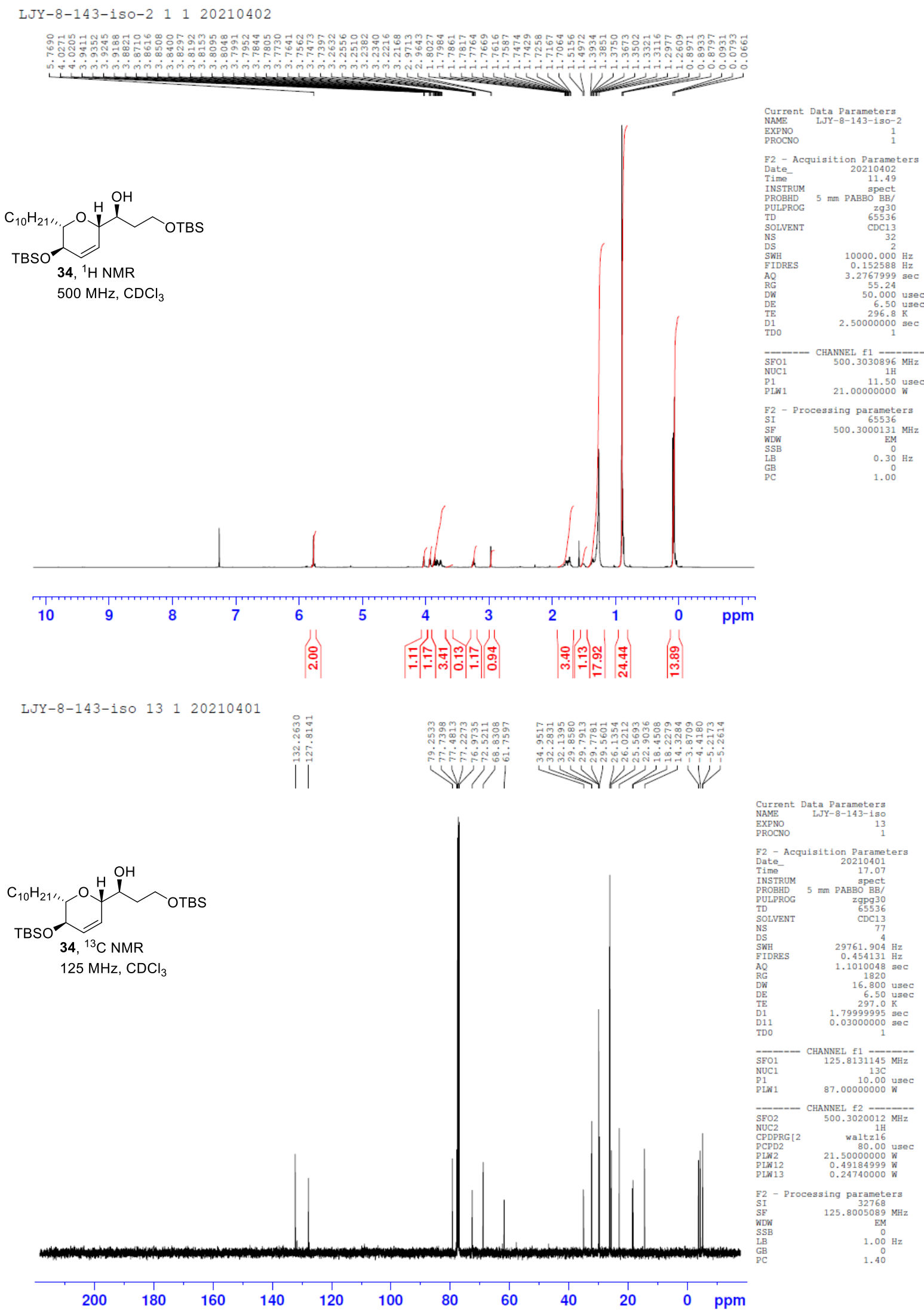




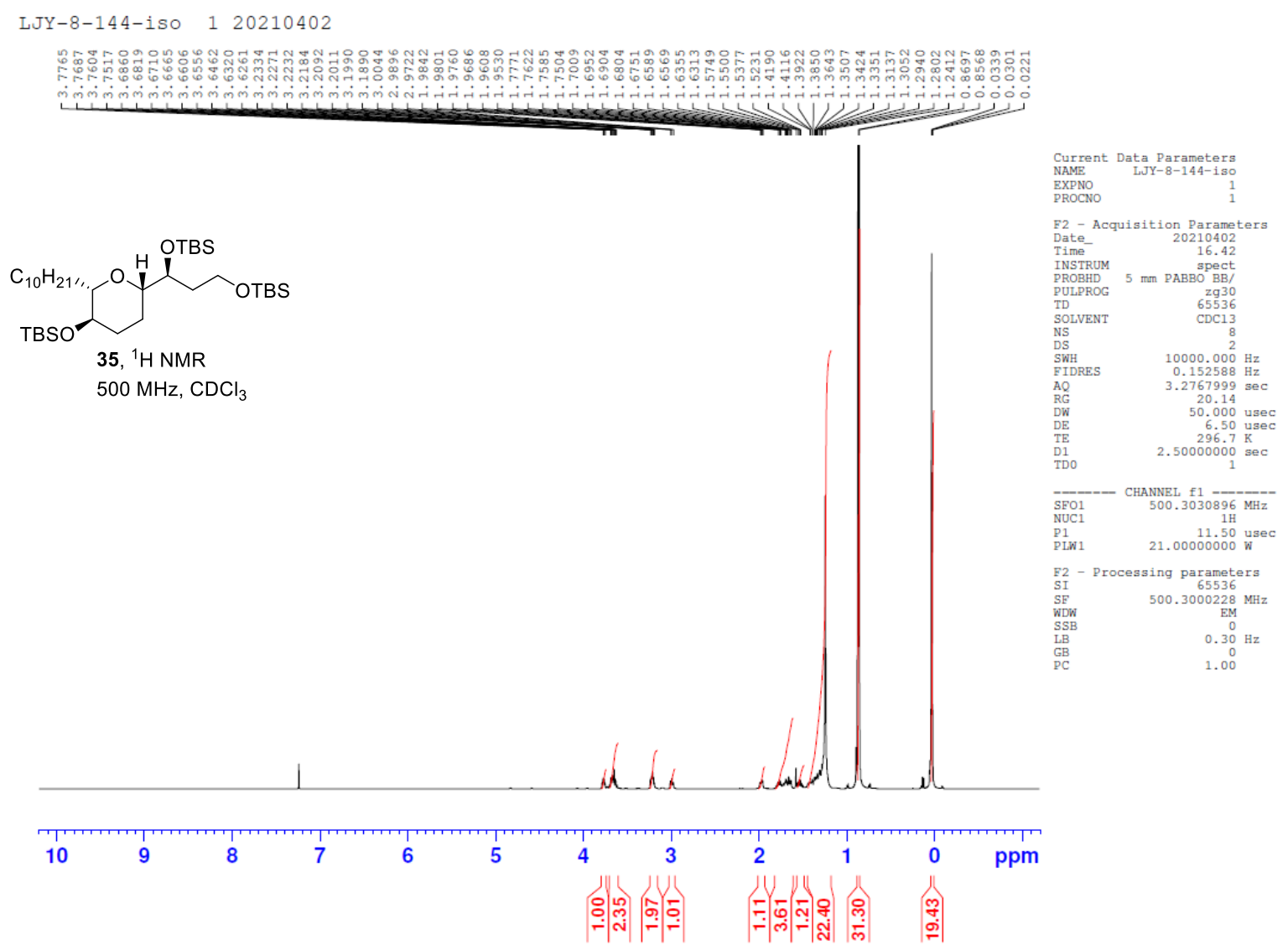

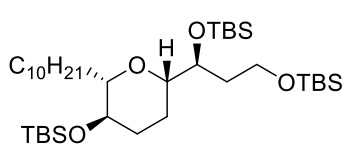

$35,{ }^{13} \mathrm{C}$ NMR $125 \mathrm{MHz}, \mathrm{CDCl}_{3}$

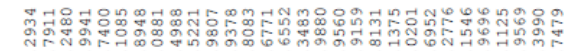

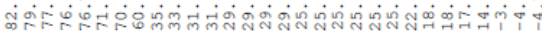

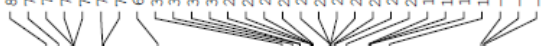
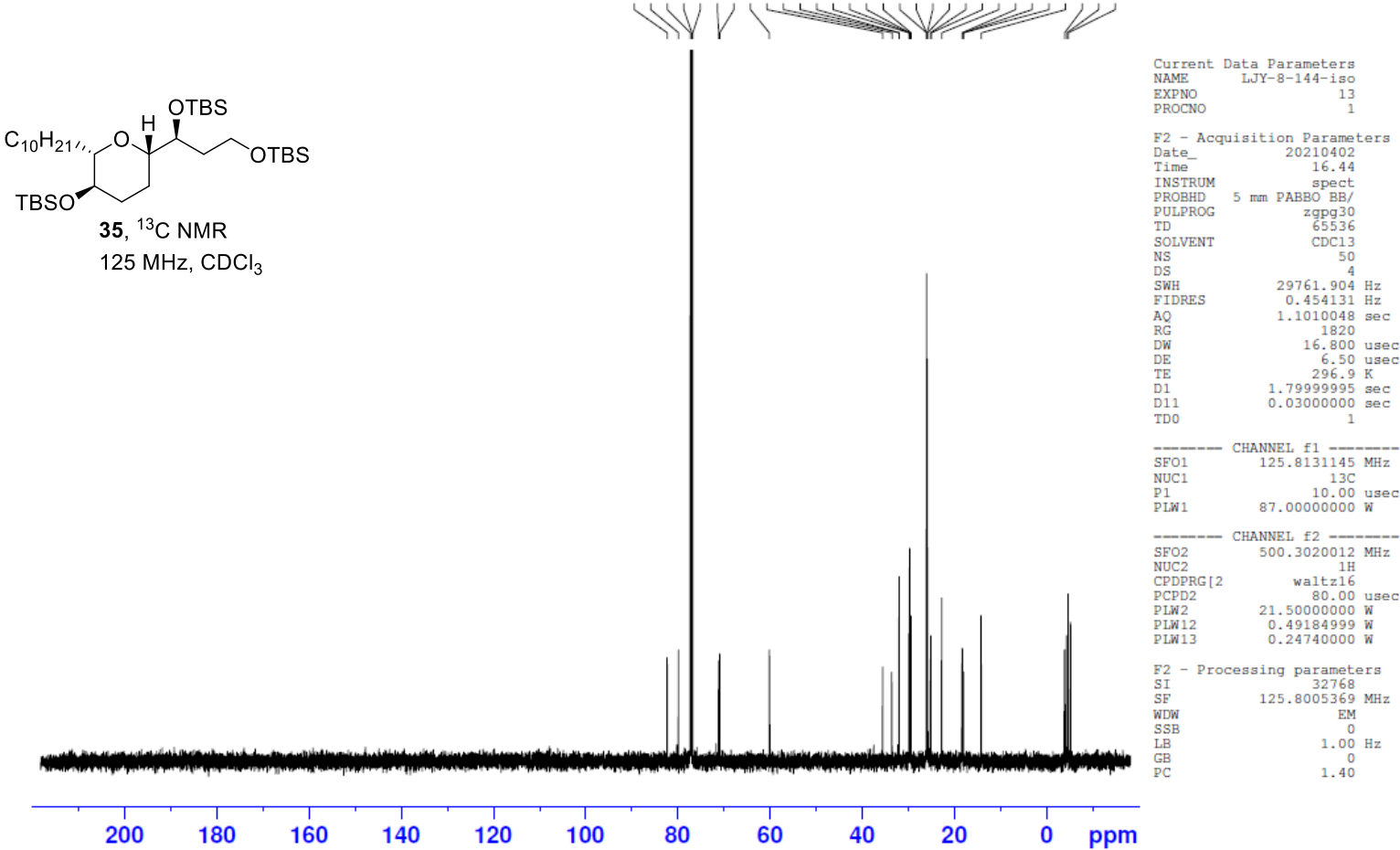


\section{0. ${ }^{1} \mathrm{H}$ NMR of Crude mixture: Determination of d.r for the addition reaction}

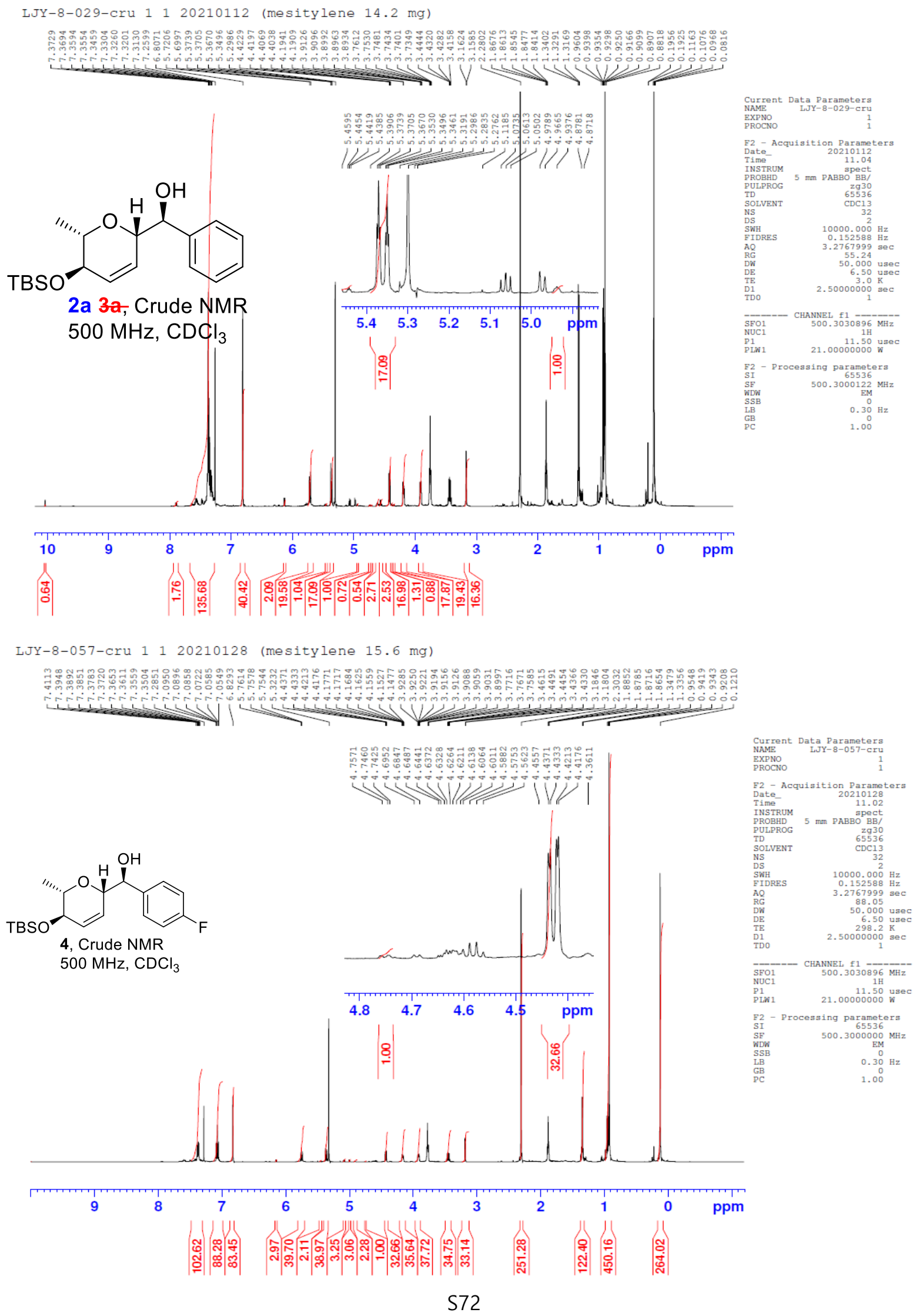



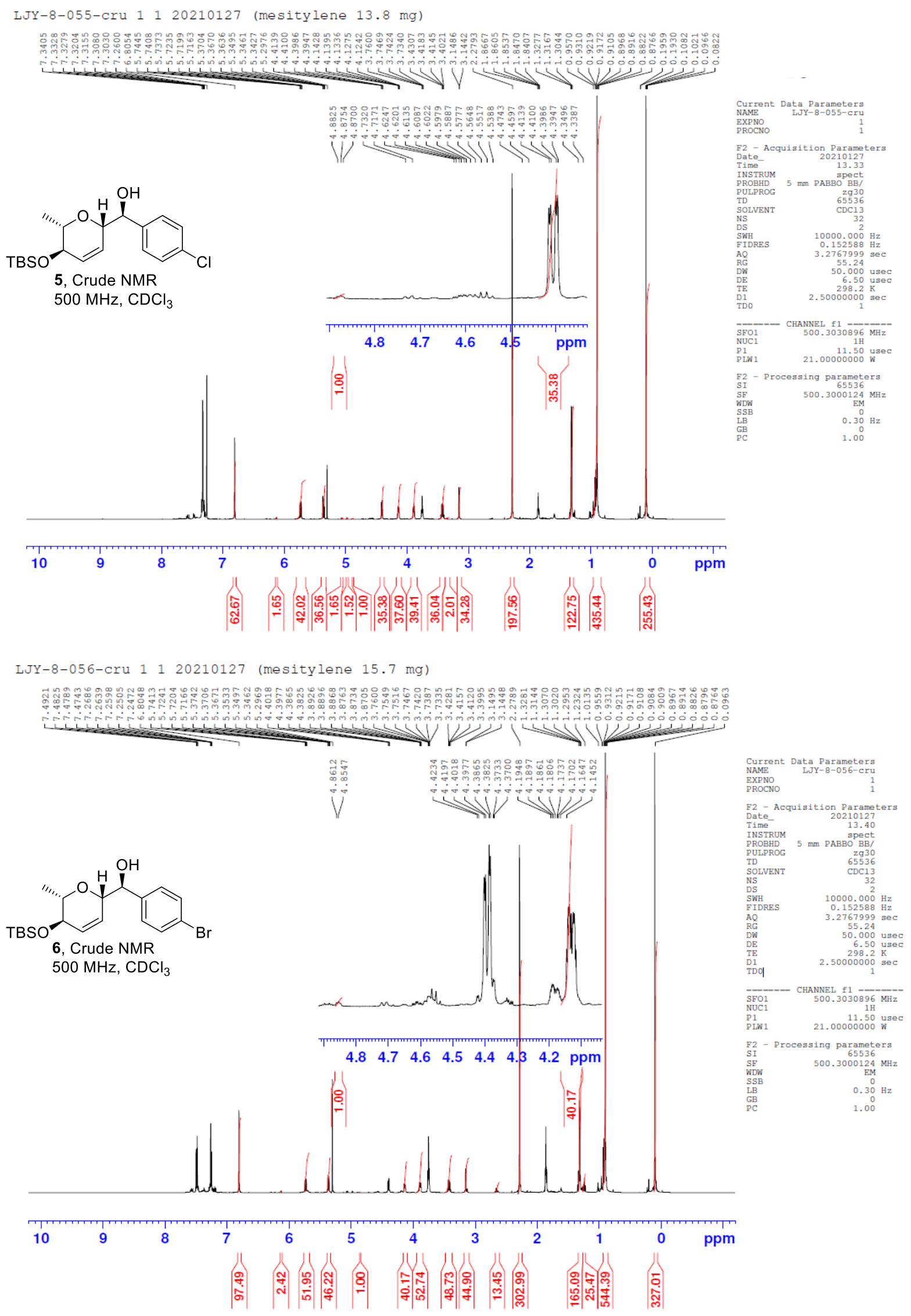


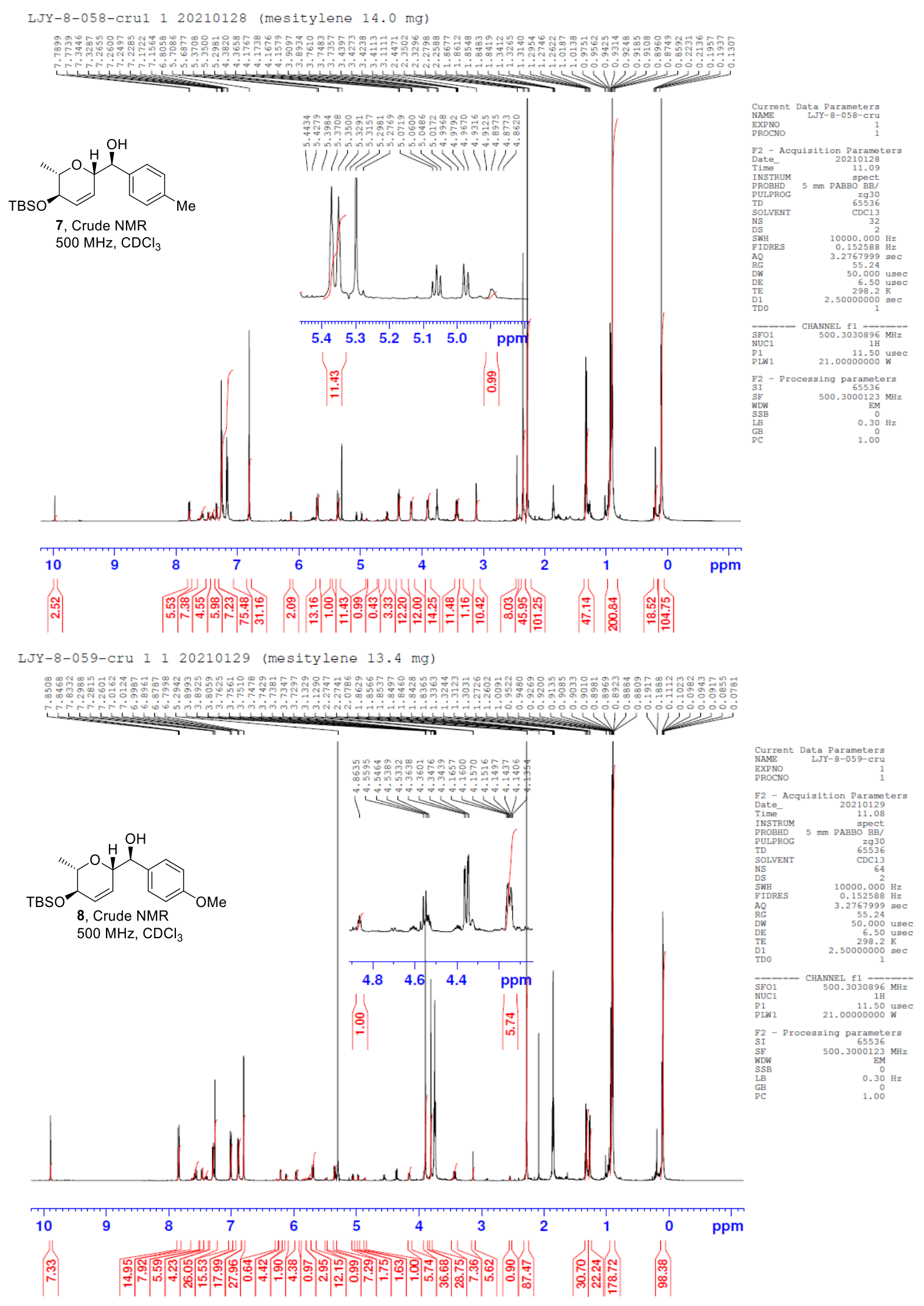




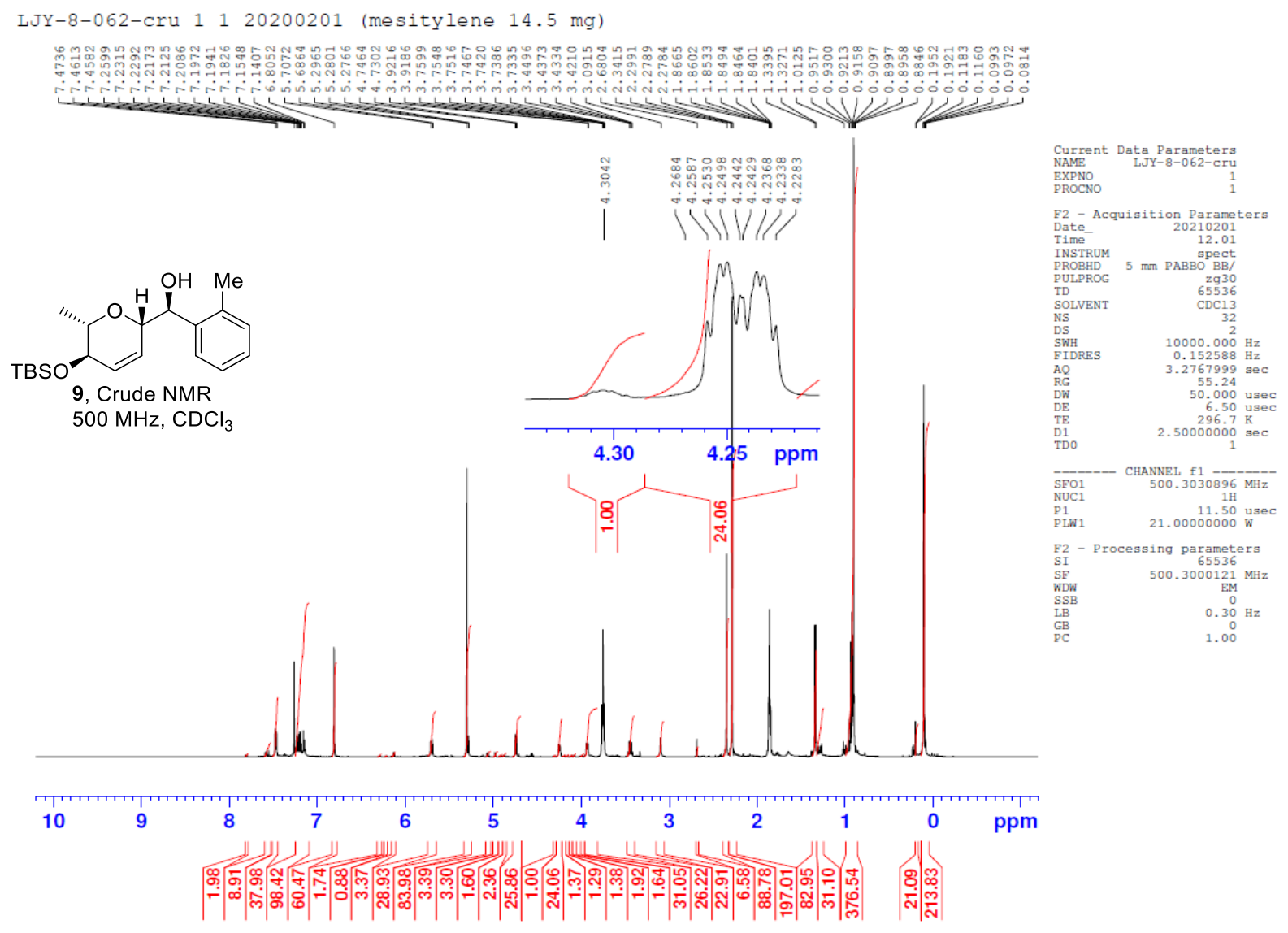

LJY-8-063-iso-\#8-15 1120210201

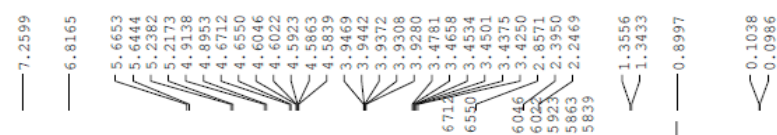

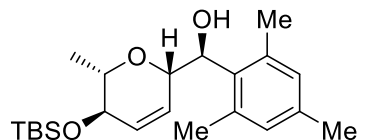

10, ${ }^{1} \mathrm{H}$ NMR

$500 \mathrm{MHz}, \mathrm{CDCl}_{3}$
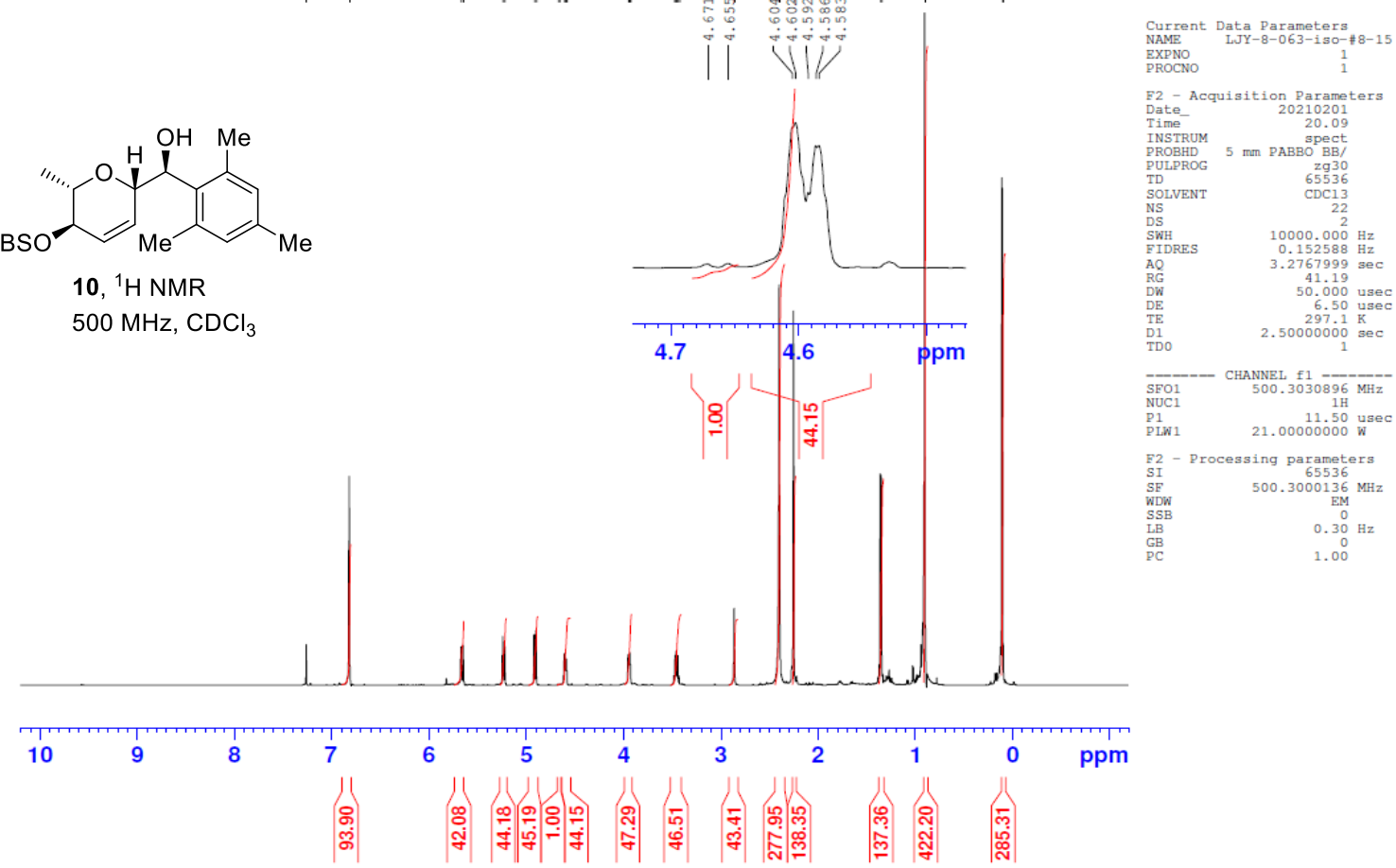

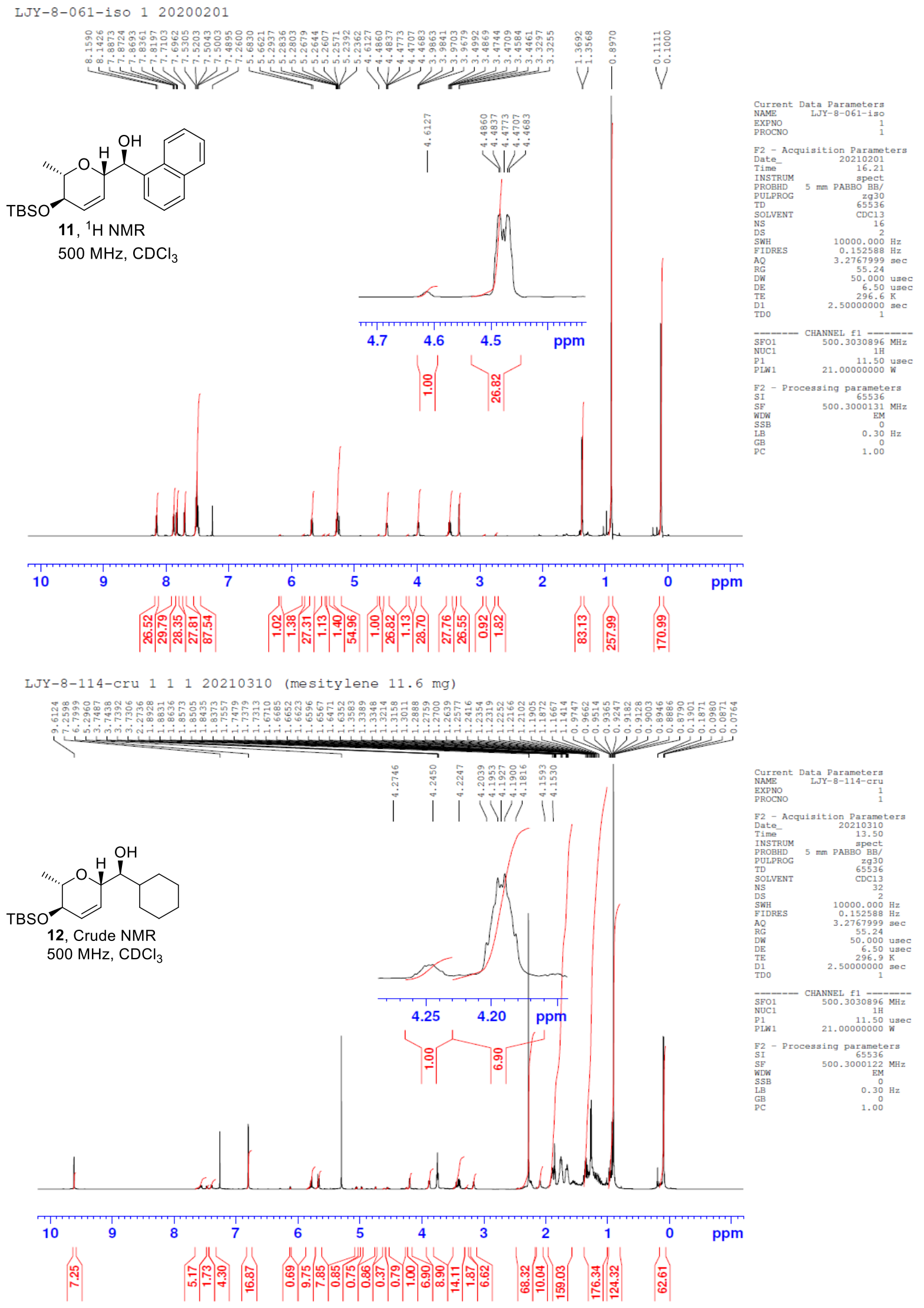

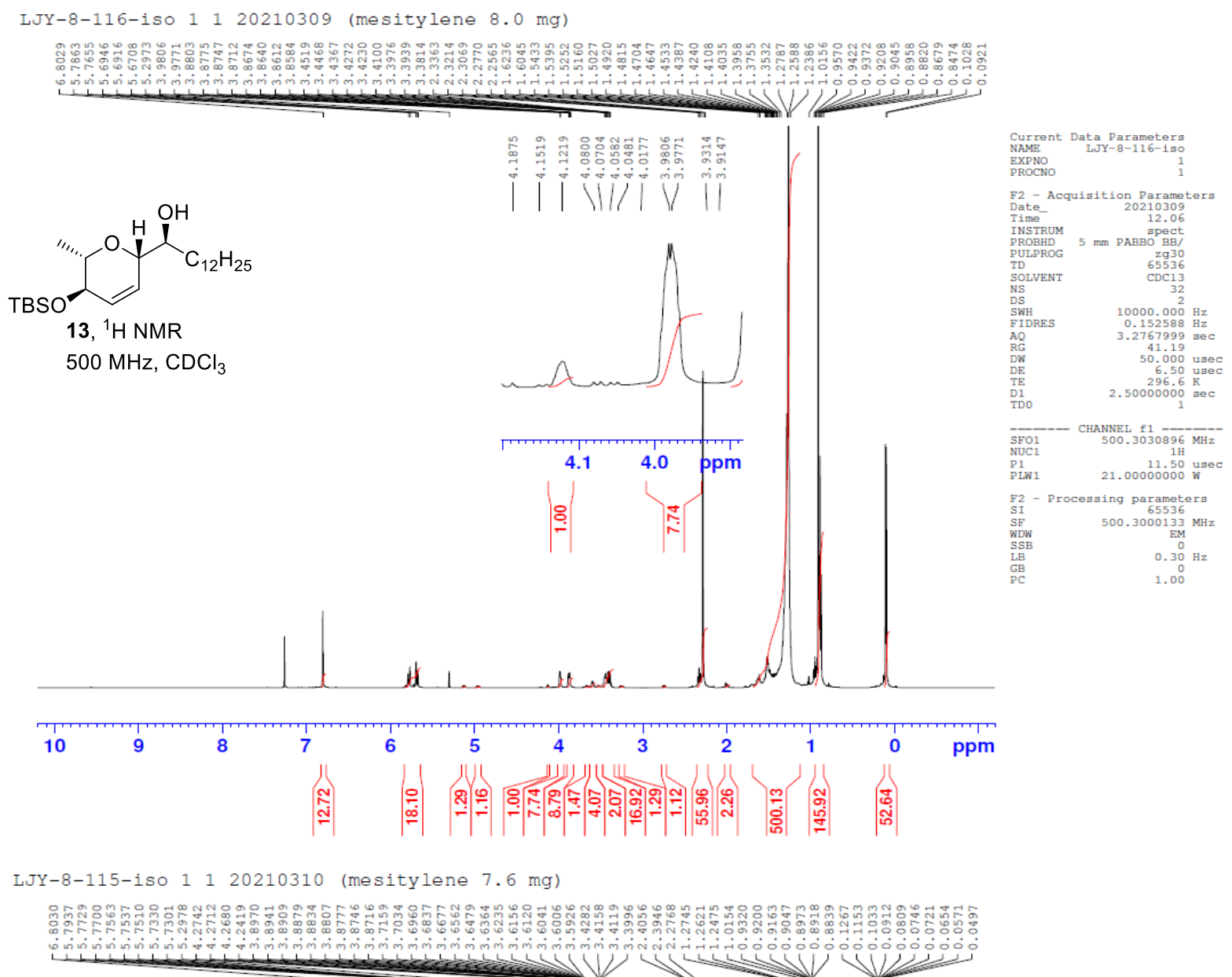<smiles></smiles>

14, ${ }^{1} \mathrm{H}$ NMR $500 \mathrm{MHz}, \mathrm{CDCl}_{3}$

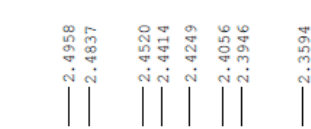

Current Data Parameters
NAME
LJY-8-115-iso EXPNO
PROCNO

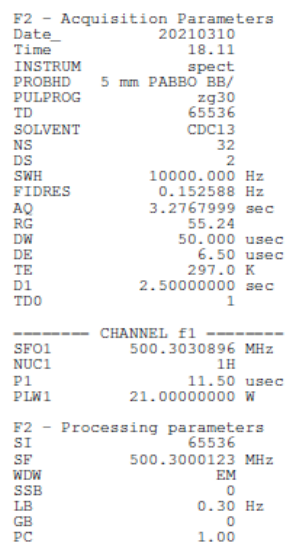

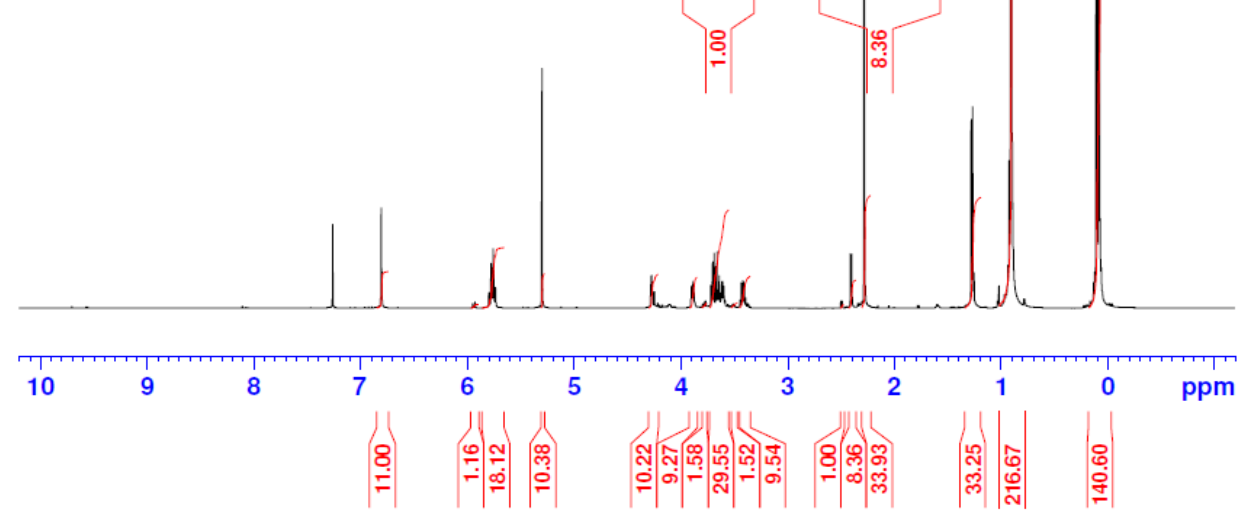



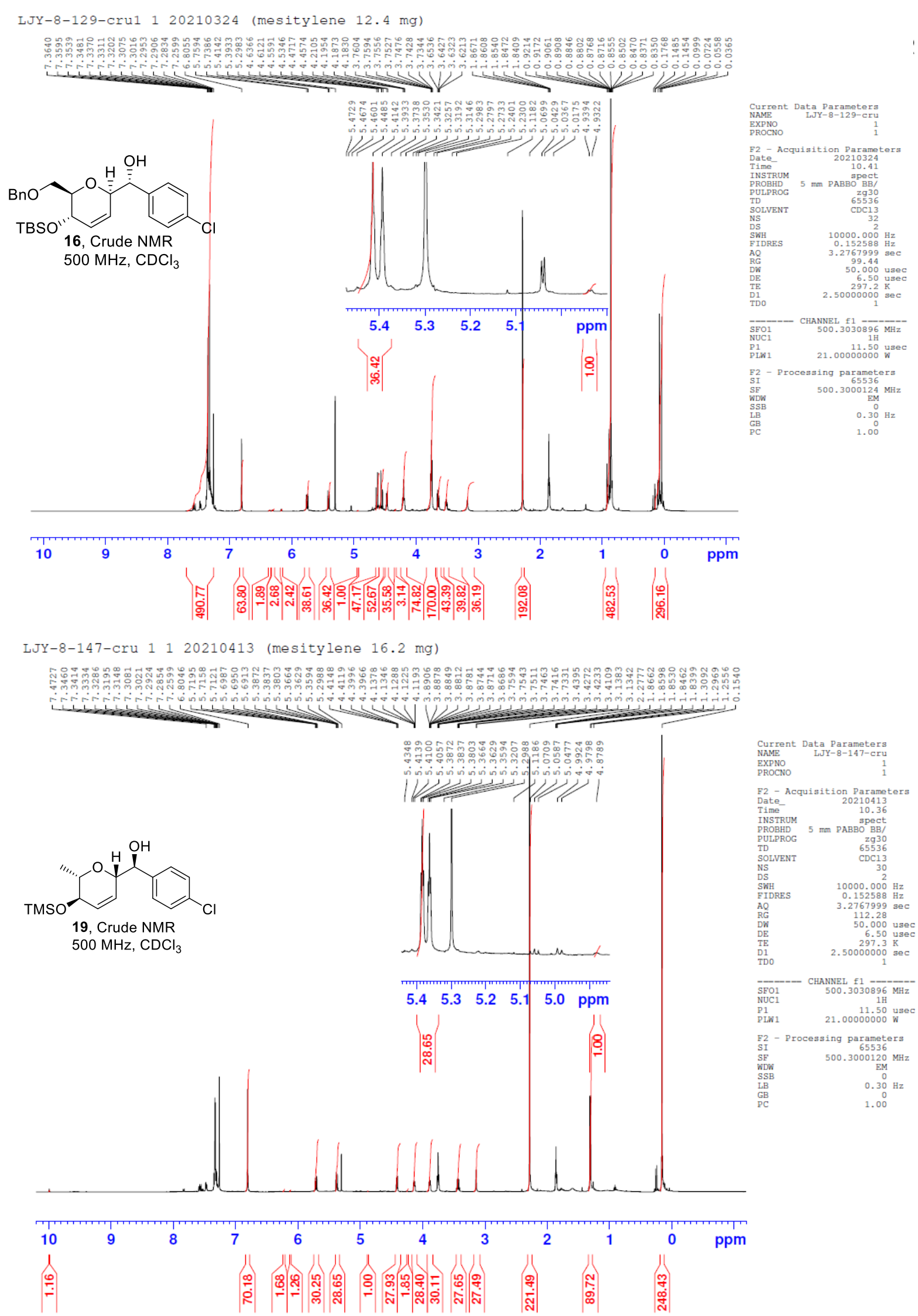


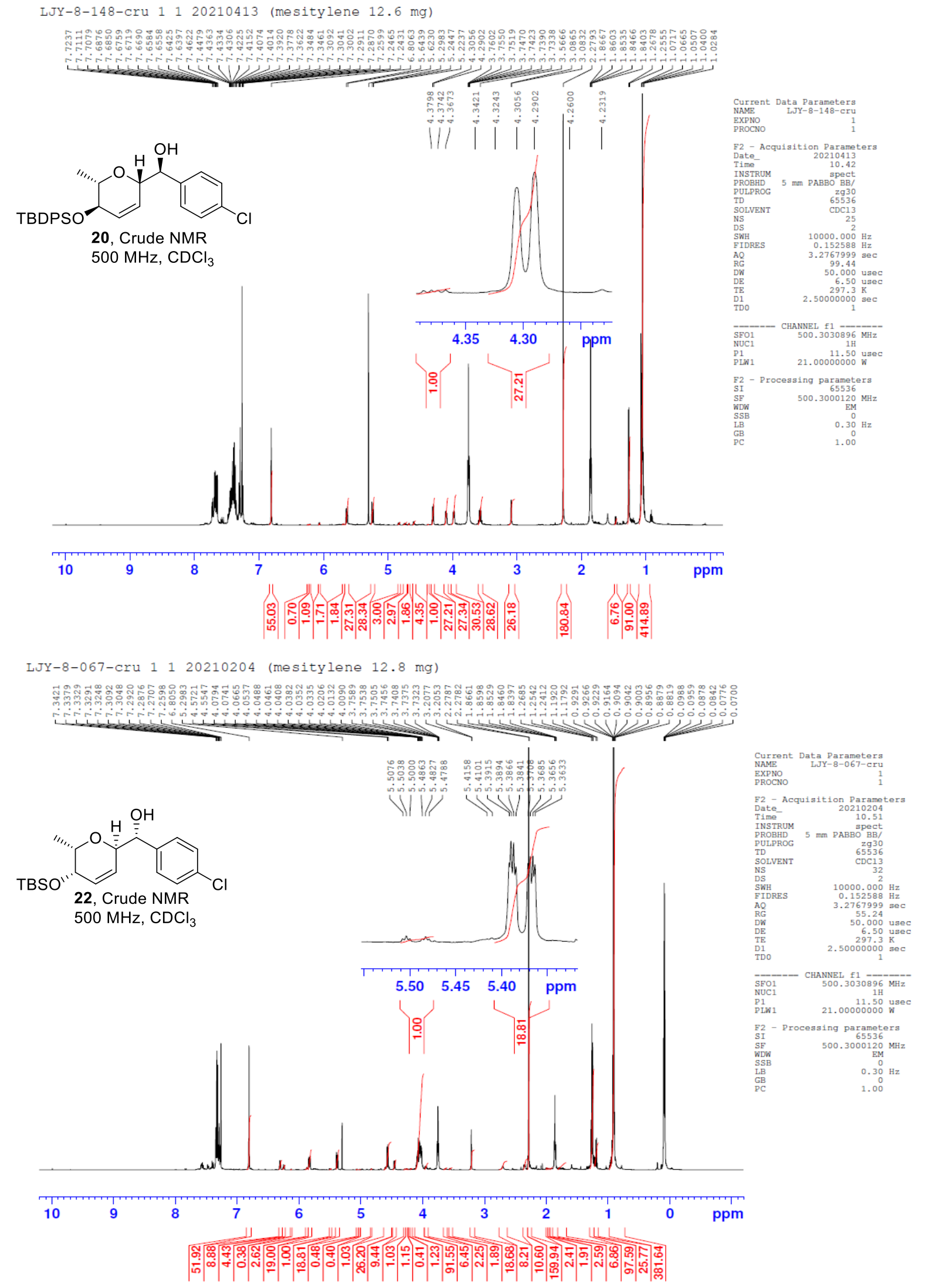




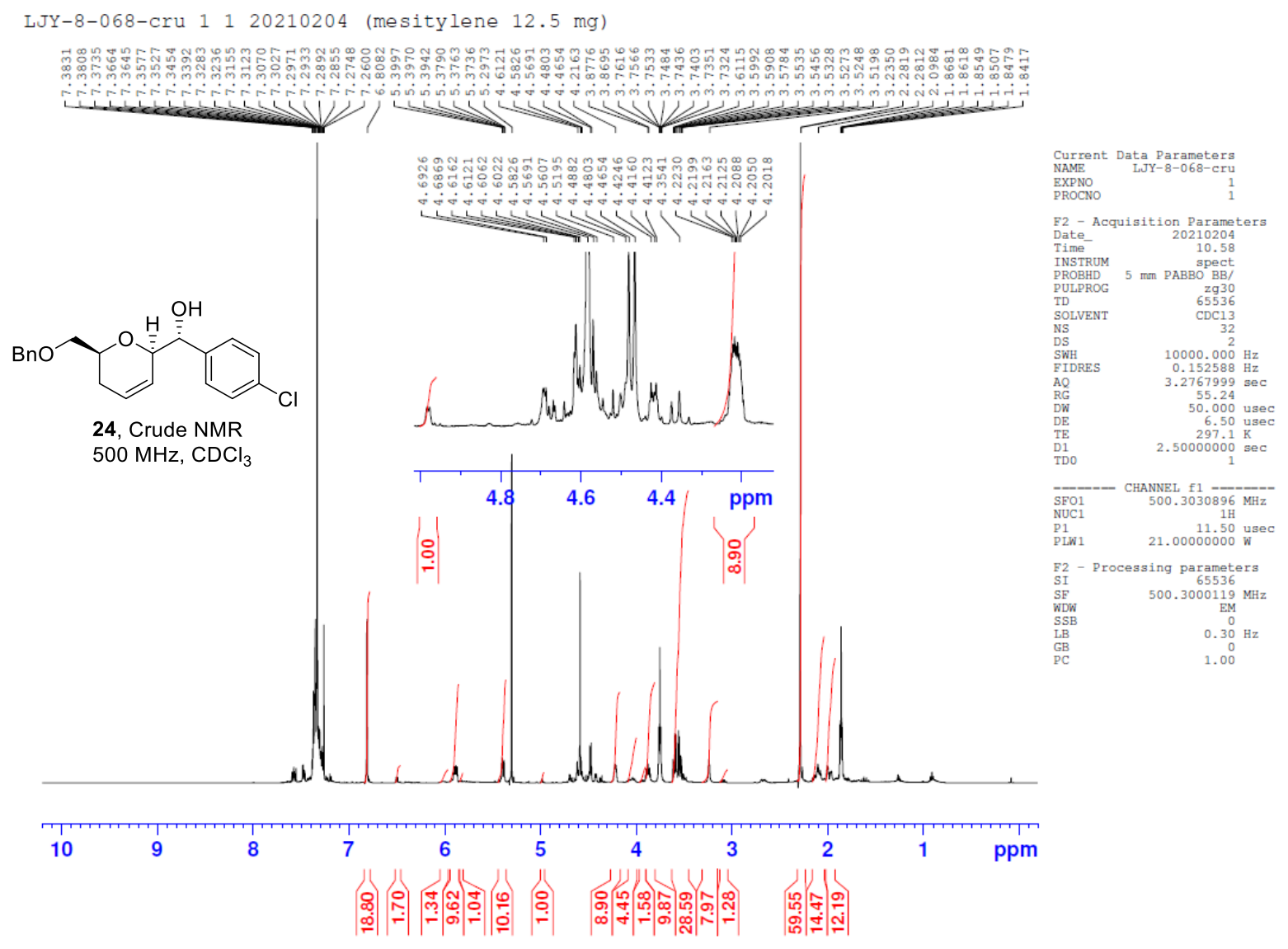

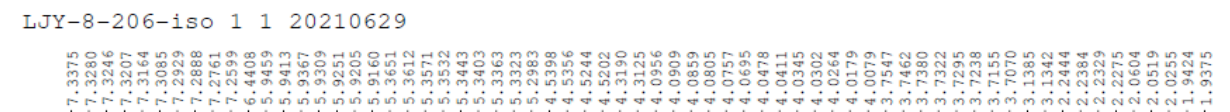

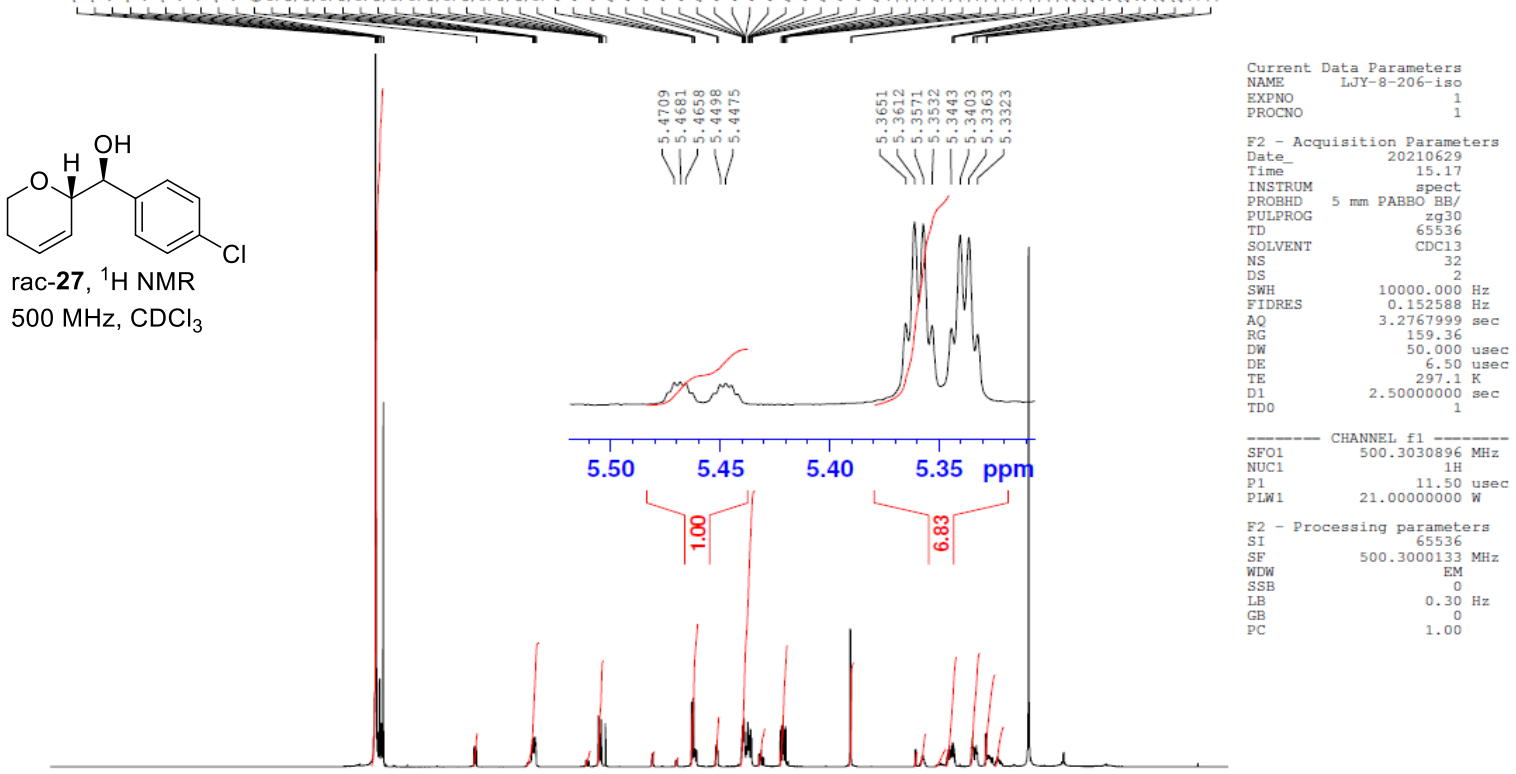

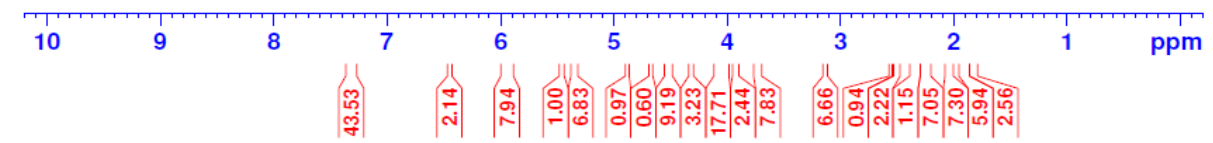

